\title{
Thermodynamics of Heisenberg Chains Coupled to Phonons
}

\author{
Dissertation \\ zur Erlangung des akademischen Grades eines \\ Doktors der Naturwissenschaften \\ des Fachbereichs Physik \\ der Universität Dortmund
}

vorgelegt von

Rainer Kühne

Dortmund

2001 


\section{Contents}

1 Introduction $\quad 1$

1.1 Motivation . . . . . . . . . . . . . . . . . 1

1.2 Spin models . . . . . . . . . . . . . . . . . . . 3

1.3 Spin-Peierls phase transition . . . . . . . . . . . . . . 4

2 Quantum Monte Carlo methods and applications 5

2.1 Quantum Monte Carlo methods . . . . . . . . . . . . . . . 5

2.2 Application to the XXZ-model . . . . . . . . . . . . . 8

2.3 Beard-Wiese algorithm . . . . . . . . . . . . . . 11

2.4 Dimerized model . . . . . . . . . . . . . . . 18

3 Heisenberg chain coupled to Einstein phonons $\quad 26$

3.1 The spin-Peierls transition in $\mathrm{CuGeO}_{3} \ldots \ldots \ldots \ldots$

3.2 Introduction of the Heisenberg model coupled to phonons . . . . . . . . 27

3.3 Approximations of the model . . . . . . . . . . . 27

3.4 The modified loop algorithm . . . . . . . . . . . . . 29

3.5 Effects of finite size and finite Trotter number . . . . . . . . . . . 30

4 Thermodynamic properties $\quad 42$

4.1 Magnetic susceptibility . . . . . . . . . . . . . . . . . . 42

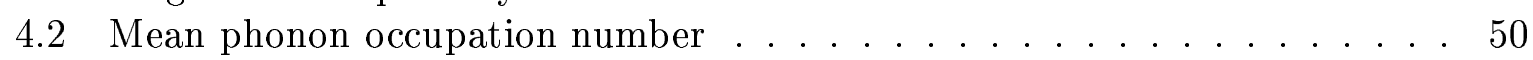

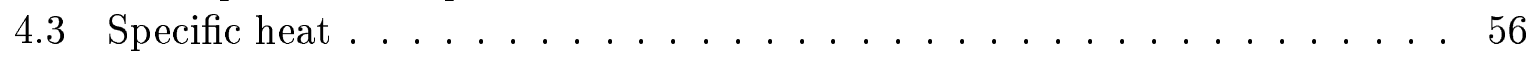

4.4 Local displacement . . . . . . . . . . . . . . . . . . . . 59

4.5 Phase diagram . . . . . . . . . . . . . . . . . 62

4.6 Correlation function . . . . . . . . . . . . . 63

4.7 Correlation length . . . . . . . . . . . . . . 70

$\begin{array}{llr}5 & \text { Two-frequency model } & 89\end{array}$

6 Conclusions $\quad 95$ 


\section{Chapter 1}

\section{Introduction}

\subsection{Motivation}

The properties of various magnetic materials can be successfully described by one-dimensional spin-1/2 isotropic antiferromagnetic Heisenberg quantum spin systems with temperature independent spin-spin coupling. Prominent examples are $\mathrm{Cu}$ (L-alanin) ${ }_{2}$ (Refs. [1, 2]), $\mathrm{Sr}_{2} \mathrm{CuO}_{3}$ (Refs. [3-5]), and $\mathrm{SrCuO}_{2}$ (Ref. [3]). Also the high-temperature phase of the spin-Peierls material $\mathrm{CuGeO}_{3}$ can be described by the frustrated one-dimensional spin- $1 / 2$ isotropic antiferromagnetic Heisenberg quantum spin system (Ref. [6]).

However, the successful description of a real system at finite and especially high temperatures has to include the phononic degrees of freedom which contribute significantly to the thermodynamic properties. In particular, the spin-phonon coupling, together with the original spin-spin coupling $J$, generates an effective spin-spin coupling which in contrast to the original spin-spin coupling is temperature dependent [7]. At low temperatures the spin-phonon interactions are expected to generate phase-transitions like the spin-Peierls transition $[8,9]$.

The aim of this study is to investigate the effects generated by the spin-phonon interaction. We will examine how the spin-phonon interaction changes the properties of the system with respect to those of the pure spin system.

In particular, we are interested whether a mapping of the experimental magnetic susceptibility of real materials onto the magnetic susceptibility (as a function of temperature) of the spin-phonon model yields information about the phonon frequencies and the spinphonon couplings of the real systems.

Another important question is whether in these models phonons exist at zero temperature and how the spin-phonon interaction influences the specific heat of the model.

Furthermore, we will investigate under which conditions the spin-phonon interaction generates a structural phase transition which corresponds to the spin-Peierls transition. Such a transition manifests itself in the appearance of dimerization generated by the phonon displacement operator $b_{l}^{\dagger}+b_{l}$, in a step-like behavior with step-width of two lattice units in the staggered correlation function, and in a spin-gap in the magnetic susceptibility below a certain temperature.

In particular, this study may be of interest for future investigations which will try to 
understand the appearance of spin-Peierls phase transitions in more complicated higher dimensional models. Spin-Peierls phase transitions exist only in three-dimensional systems, because according to the Mermin-Wagner theorem [10], long-range (antiferromagnetic) order at nonzero temperature is possible only in three-dimensional systems. KosterlitzThouless phase transitions [11], which can exist in two-dimensional systems which are of topological order only (i. e. of long-range order of non-conventional type), cannot occur in Heisenberg systems.

We restrict our study to a very special and simple model, namely the one-dimensional spin-1/2 isotropic antiferromagnetic Heisenberg quantum spin system with nearest neighbour interaction which is coupled to one-dimensional dispersionless phonons. This restriction is necessary because of conceptual problems such as the minus sign problem and difficulties to find an appropriate algorithm.

This work is organized as follows. We start with a brief history of the spin models and the spin-Peierls phenomenon in sections 1.2 and 1.3. In chapter 2 we present a short review of the quantum Monte Carlo methods which have been developped since 1977. We proceed by applying a very efficient quantum Monte Carlo method, the loop algorithm, to the XXZ Heisenberg model and the dimerized model. Our aim is to study the magnetic susceptibility and the specific heat of these systems. Furthermore, we apply the continuous version of the loop algorithm on the isotropic Heisenberg model. To test the efficiency of this algorithm we determine the magnetic susceptibility and the specific heat of this system and compare our results with the exact values calculated by Klümper [19]. Moreover we study the number of transition times of the isotropic Heisenberg model as a function of the temperature. At low temperatures this number will turn out to be proportional to the inverse temperature.

In chapter 3 we will introduce a modification of the discrete version of the loop algorithm. This modification includes the update procedure for the phononic degrees of freedom by considering both the spin-phonon coupling and the free phonons. By using this modified loop algorithm we are able to simulate an isotropic Heisenberg model coupled to Einstein phonons. We point out the approximations of both this model and this algorithm and justify their suitability. The approximations of the algorithm include effects of finite size and finite Trotter number. These effects are studied in detail.

In chapter 4 we report on a simulation of the one-dimensional spin- $1 / 2$ isotropic antiferromagnetic Heisenberg model coupled to Einstein phonons. The spin-spin interaction includes nearest neighbor interaction only. We study in detail the magnetic susceptibility, the effective spin-spin coupling, the probability of the phonon occupation, the specific heat, and the local displacement. By determining the appearance of dimerization at low temperatures we present a phase diagram of the model. By examining the spin-spin correlation we determine the behavior of the correlation length as a function of the temperature in dependence on the phonon frequency and the spin-phonon coupling.

Finally, in chapter 5 we study the two-frequency model. This model is of particular interest for the understanding of $\mathrm{CuGeO}_{3}$, because the spin-Peierls phase transition of this material is generated mainly by phonons of two frequencies. 


\subsection{Spin models}

The Hamiltonian of the XYZ-model or generalized (one-dimensional) Heisenberg model (with nearest neighbour interaction) is,

$$
\mathcal{H}=\sum_{l=1}^{N}\left(J_{x} S_{l}^{x} S_{l+1}^{x}+J_{y} S_{l}^{y} S_{l+1}^{y}+J_{z} S_{l}^{z} S_{l+1}^{z}\right),
$$

where $S$ denotes the spin operator. Heisenberg [12] suggested the isotropic model, i. e. that with $J_{x}=J_{y}=J_{z}$, and was the first to investigate it. By using this model, he was able to explain the Weiss molecular forces by the quantum mechanical exchange interaction of the spins. Bethe [13] introduced a method for the calculation of the eigenfunctions in zeroth and the eigenvalues in first order of the approximation method of London and Heitler. By using this "Bethe-ansatz" he solved the isotropic model exactly. Subsequently, Hulthén [14] calculated with the Bethe-ansatz the energies of the ground state and the first excited state.

With the help of the Bethe-ansatz, Orbach [15] determined the energy eigenvalues of the one-dimensional Heisenberg model for chain lengths up to $N=10$. Des Cloizeaux and Pearson [16] computed the lowest excitations of this system. Finally, Bonner and Fisher [17] evaluated the complete thermodynamics (especially the magnetic susceptibility and the specific heat) of this model. Eggert et al. [18] determined the magnetic susceptibility with high precision with the help of the Bethe ansatz solution to the quantum transfer matrix. Furthermore, Klümper calculated the complete thermodynamics with very high precision with the help of an approach to the thermodynamics on the basis of the physical excitations (spinons) [19]. In a recent study, he did this over the very large temperature range $10^{-24} \leq T / J \leq 10[20]$.

Anisotropic Heisenberg models have also been investigated. In the pioneering work of Lieb, Schultz, and Mattis [21] the one-dimensional antiferromagnetic model with $J_{x} \neq$ $J_{y}$ and $J_{z}=0$ (XY-model) was investigated. They determined its ground state, all elementary excitations, and the free energy. They showed the XY-model to have a longrange order (in contrast to the isotropic model). This discovery is named "Lieb-SchultzMattis theorem". Katsura [22] calculated the partition function and the free energy of the XY-model in the external magnetic field. Furthermore, he calculated both the magnetic susceptibility and the specific heat for three models: (i) $J_{x}=J_{y}$ and $J_{z}=0$, (ii) $J_{x}=J_{y}=0$ and $J_{z} \neq 0$, (iii) $J_{x}=J_{y}=J_{z}$. (He studied the case (iii) by the high-temperature expansion and by analysing small systems). Yang and Yang [23, 24, 25] examined the XXZ-model $\left(J_{x}=J_{y} \neq J_{z}\right)$ with the Bethe-ansatz. Lieb [26] showed the equivalence between the XXZ-model and the 6-vertex model and calculated the entropy of the two-dimensional "ice-model" by using the transfer matrix method. Moreover he solved the Rhys F model [27] and the two-dimensional Slater KDP model [28] exactly. According to $\mathrm{Wu}[29]$ the two-dimensional Slater KDP model experiences a ferromagnetic phase transition of second order, where for the substance $\mathrm{KH}_{2} \mathrm{PO}_{4}$ the transition temperature is $T=123 \mathrm{~K}$. Finally, Baxter [30-33] solved the XYZ-model exactly with the help of the transfer matrix method, where he used the equivalence between this model and the 8vertex model. The XYZ-model includes as special cases the one-dimensional Ising model 
(the two-dimensional Ising model was solved by Onsager [34]), the dimer model [35], the ice model, the Rhys F model, and the Slater KDP model. Johnson et al. [36] calculated the lowest excitations of the XYZ-model. Klümper and Zittartz [37] calculated all eigenvalues of the transfer matrix of the 8-vertex model in the thermodynamic limit and used this result to determine all energy excitations of the XYZ-model. A modern formulation of the solutions of the 6 -vertex and the 8-vertex models is given in Ref. [38]. A review on these models is Ref. [39].

\section{$1.3 \quad$ Spin-Peierls phase transition}

In 1955, Peierls [40] argued that periodic lattice vibrations with wavelength of twice the lattice constant, $\lambda=2 a$, can generate a phase transition from the conducting state to the dimerized insulating ground state of a semiconductor. This phenomenon is now named "Peierls transition." McConnell and Lynden-Bell [41] suggested the magnetic analogon, where the exchange interaction between the spin generates a phase transition from the antiferromagnetic phase to the dimerized phase. This is now called the spin-Peierls phase transition. Pytte [42] and Cross and Fisher [43] examined the spin-Peierls phenomenon at the mean field level by the random phase approximation for one-dimensional spin systems coupled to three-dimensional phonons.

The spin-Peierls phase transition was experimentally verified in 1975 in organic materials with complicated crystal lattice structure [44, 45]. This phenomenon found increasing interest in the recent past, because of the discovery of a spin-Peierls transition in the inorganic material $\mathrm{CuGeO}_{3}[6]$, whose thermodynamic behavior can be described with good approximation by a one-dimensional isotropic antiferromagnetic Heisenberg-model with next nearest neighbour interaction [46-48]. The crystal structure of $\mathrm{CuGeO}_{3}$ consists of octaeders and tetraeders with the oxygen atoms at the corners, a copper atom within the center of each octaeder and a germanium atom within the center of each tetraeder $[49,50]$. Its phase transition is generated mainly by phonons of two frequencies $[51,52]$.

Possibly, $\alpha-\mathrm{NaV}_{2} \mathrm{O}_{5}$, whose magnetic susceptibility for high-temperatures can be well decribed by the one-dimensional isotropic antiferromagnetic Heisenberg system with nearest neighbour interaction [53], is a second inorganic spin-Peierls material with simple crystal structure [53-55]. However, recent X-ray structure data analysis [56, 57] and Raman experiments [58] show $\alpha-\mathrm{NaV}_{2} \mathrm{O}_{5}$ to be rather a quarter-filled ladder than a onedimensional Heisenberg system. So new scenarios for the phase-transition have to be taken into consideration. Recent observations suggest a charge ordering and spin-Peierls transition [59].

Experimentally, the spin-Peierls phase transition can be identified if the following observations are satisfied simultaneously [6]:

- Dimerization of two neighboured spins and therefore doubling of the unit cell.

- Two neighboured spins pair each to a spin singlet. Hence, a gap appears in the magnetic susceptibility.

- A peak appears in the specific heat, because the phase transition is of second order. 


\section{Chapter 2}

\section{Quantum Monte Carlo methods and applications}

\subsection{Quantum Monte Carlo methods}

The underlying idea of quantum Monte Carlo methods is to map a $d$-dimensional quantum system onto a $d+1$-dimensional classical system. This becomes possible by application of the Trotter-Suzuki equation [60],

$$
\exp \left(\sum_{l=1}^{N} A_{l}\right)=\lim _{M \rightarrow \infty}\left(\mathrm{e}^{A_{1} / M} \cdot \ldots \cdot \mathrm{e}^{A_{N} / M}\right)^{M},
$$

where the non-Abelian operators of the left hand side can be expressed by the commuting operators of the right hand side in the limit $M \rightarrow \infty$. With the help of this formula, Suzuki et al. [61] invented the first quantum Monte Carlo algorithm and applied it to the one-dimensional XY-model.

Hirsch et al. [62-64] invented the worldline algorithm for fermionic systems, where the fermions are located on the sites of the lattice. They also suggested to describe systems with both fermionic and bosonic degrees of freedom by this algorithm. In later studies, they used this idea and applied the worldline algorithm to electron-phonon systems, the Su-Schrieffer-Heeger model [65], the molecular crystal model [66], and a model to examine the Peierls instability [67].

Marcu and Wiesler [68] applied the worldline algorithm to spin models, especially to the one-dimensional XXZ-model. They showed the magnetic susceptibility to depend linearly on the inverse Trotter number squared.

The first non-local update procedure, where whole clusters are updated, so that the autocorrelation times are reduced significantly and critical slowing down is reduced, was introduced by Swendsen and Wang [69]. This algorithm was applied to the Potts model [69] and the classical two-dimensional XX-model [70].

From then on further cluster algorithms were invented [71-77] [71, 72, 73, 74, 75, 76, 77] and applied to the two-dimensional XXX-model [71, 72, 76, 77]. A new invention were the loop updates [76, 77], which gave birth to the loop algorithm [78-80] and its continuous 
version [81], i. e., its limit $M \rightarrow \infty$. In this work we will use both the discrete and the continuous version of the loop algorithm.

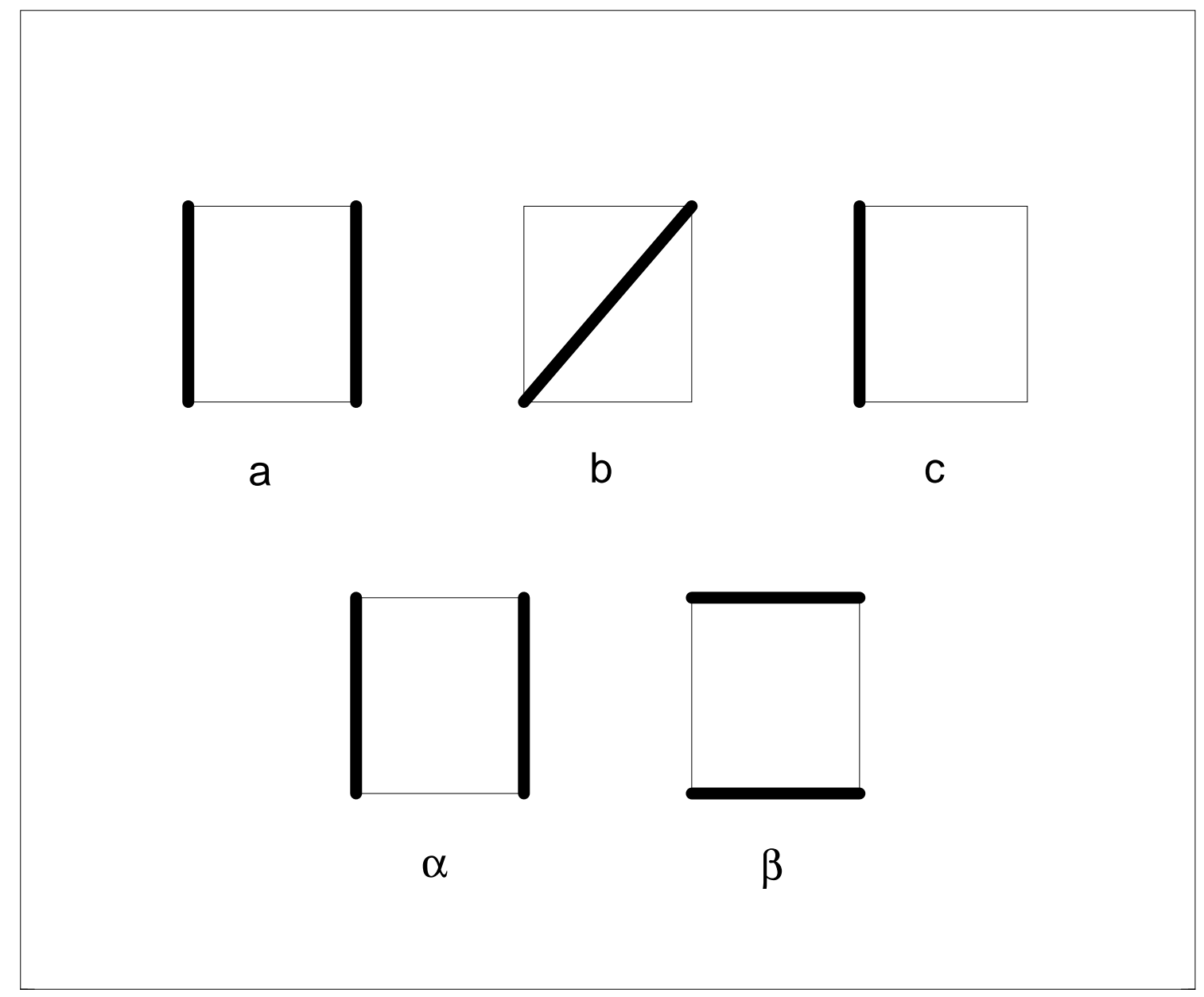

Figure 2.1: Plaquettes (upper row) and breakups (lower row) of the loop algorithm which are required for a quantum Monte Carlo algorithm of the XXX Heisenberg model. The spins are located at the corners of the plaquette. The thick lines denote the worldlines (connections of the up-spins). Naturally, for each of these plaquettes there exists a counterpart, where up- and down-spins are interchanged, so that we have in total six plaquettes. In the lower row (breakups), the thick lines denote the directions which the loops have to follow.

The loop algorithm introduced by Evertz et al. $[78,80]$ is an elegant method for the simulation of a $d$-dimensional quantum spin system with nearest neighbour interaction. This algorithm maps the $d$-dimensional quantum system onto a $d+1$-dimensional classical spin system with periodic boundary conditions (to satisfy translation invariance). The loop algorithm has several advantages as compared to the conventional world line algorithm. The autocorrelations are drastically reduced, because the spin updates are 
(a)

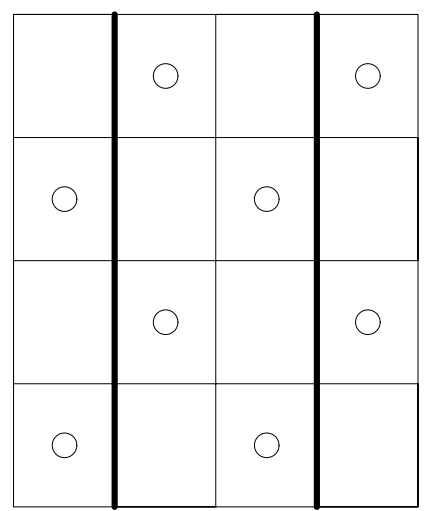

initial spin configuration (b)

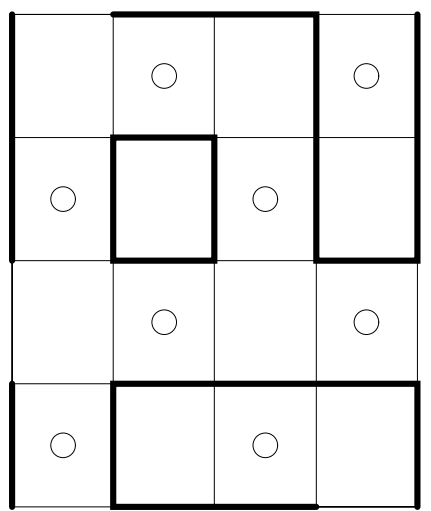

breakup configuration (c)

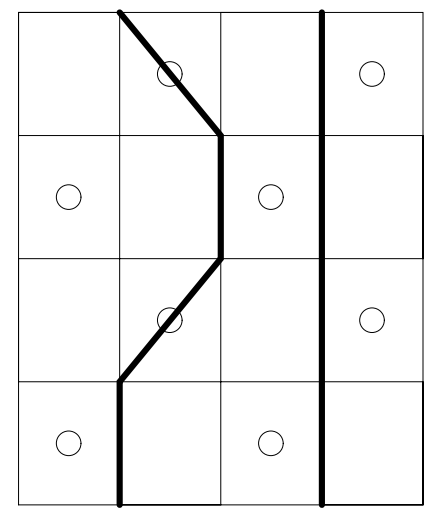

new spin configuration

Figure 2.2: Update procedure of the discrete loop algorithm. We consider here a chain with $N=4$ lattice sites, Trotter number $M=2$, and periodic boundary conditions. The Trotter time is the ordinate. Interaction plaquettes are denoted by circles in the middle of the plaquettes. In this example, we start with an initial spin configuration with zero magnetization (a). We attribute a breakup to each interaction plaquette. For the XXX model breakup $\alpha$ is attributed to plaquette $a$, breakup $\beta$ is attributed to plaquette $b$. For plaquette $c$ we have to choose either $\alpha$ or $\beta$. Figure (b) shows the breakup pattern which consists of two loops. Let us flip the smaller loop, i. e. flip its spins. The result is a new spin configuration, shown in (c). The transition from (a) to (c) is called a spin-update.

performed globally. This results in a substantial reduction of the required number of quantum Monte Carlo sweeps. Moreover the variation of both the winding number and the magnetization is automatically included in the update procedure.

For each quantum Monte Carlo step a spin update is generated as follows. Start with a given "initial" spin configuration, where at each lattice site there is exactly one classical spin. The Hamiltonian acts locally on individual interaction plaquettes. Hence, for each 
interaction plaquette one can choose a certain "breakup" plaquette. The probability for the breakups depends only on the spin configuration of the considered plaquette, i. e., this is a local process. One thus covers the whole lattice with a breakup pattern. The graphs defined by the breakups constitute a set of loops, where each spin is attributed to exactly one loop. Because of the zero divergence criterion the loops always close. For the isotropic Heisenberg chain without exterior magnetic field the loops can be flipped separately with the probability 1/2. The update procedure is shown in Fig. 2.2.

The Boltzmann weights of the plaquettes for the isotropic Heisenberg chain (cf. Fig. 2.1) are,

$$
\begin{aligned}
a & =\mathrm{e}^{-\beta J /(2 M)} \\
b & =\mathrm{e}^{\beta J /(2 M)} \sinh \frac{\beta J}{M} \\
c & =\mathrm{e}^{\beta J /(2 M)} \cosh \frac{\beta J}{M} .
\end{aligned}
$$

The breakup-procedure becomes more complicated for the XXZ-model than for the XXX-model. While the XXX-model needs only two breakups, the XXZ-model requires a third breakup for the anisotropy parameter $|\Delta|<1$. For $|\Delta|>1$ a fourth breakup, called "freezing", is needed where all four spins of a plaquette are flipped together. This plaquette requires that the two loops corresponding to the breakup are flipped (and therefore "freezed") together.

\subsection{Application to the XXZ-model}

The loop algorithm $[78,80]$ can be applied to the anisotropic (XXZ) Heisenberg model. Its Hamiltonian is

$$
\mathcal{H}=\frac{J}{2} \sum_{l=1}^{N} \sigma_{l}^{x} \sigma_{l+1}^{x}+\sigma_{l}^{y} \sigma_{l+1}^{y}+\Delta \sigma_{l}^{z} \sigma_{l+1}^{z},
$$

where $\Delta$ denotes the anisotropy parameter and $\sigma$ is the Pauli matrix.

Quasi one-dimensional systems whose anisotropy parameter $\Delta$ differs strongly from unity have already been identified. Prominent examples are $\mathrm{CsCoCl}_{3}, \mathrm{CsCoBr}_{3}$, and $\mathrm{RbCoCl}_{3}$ with $\Delta \sim 6, \ldots, 7[82]$ and $\mathrm{Cs}_{2} \mathrm{CoCl}_{4}$ with $\Delta \simeq 0.25[83,84]$.

We examine the XXZ-model for the anisotropy parameter $0 \leq \Delta \leq 5$. The case $\Delta=0$ corresponds to the XX model which is particularly simple, because it consists only of the kinetic term and has no potential contribution. This becomes understandable by the Jordan-Wigner transformation which transforms spin-operators into fermion operators,

$$
\begin{aligned}
S_{l}^{x} S_{l+1}^{x}+S_{l}^{y} S_{l+1}^{y} & =\frac{1}{2}\left(S_{l}^{+} S_{l+1}^{-}+S_{l}^{-} S_{l+1}^{+}\right) \\
& =\frac{1}{2}\left(c_{l}^{+} c_{l+1}^{-}+c_{l}^{-} c_{l+1}^{+}\right),
\end{aligned}
$$

where $c^{+}$and $c^{-}$denote the fermion creation and annihilation operators, respectively. 


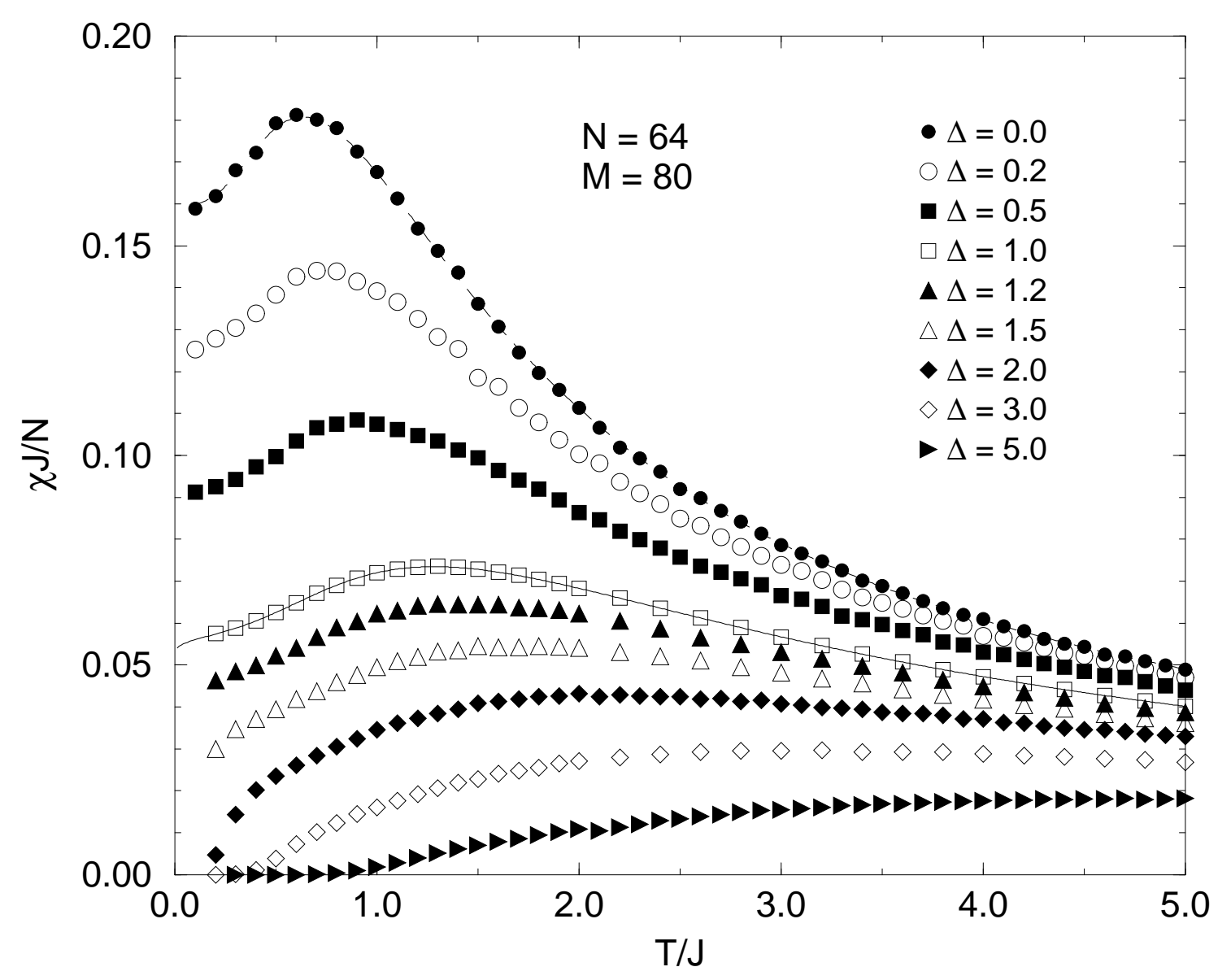

Figure 2.3: Magnetic susceptibility per site versus temperature. The anisotropy coupling constant $\Delta$ is chosen between 0.0 and 5.0. The exact data for $\Delta=1.0$ are taken from A. Klümper (isotropic model), $\Delta=0.0$ is the XX-model (the dashed line shows the analytic data). A model with $\Delta \rightarrow \infty$ would correspond to the Ising model with infinite coupling constant $J \Delta$. The chain length is $N=64$ and the Trotter number is $M=80$.

The model with $\Delta=1$ is the isotropic Heisenberg chain. Finally, the case $\Delta \rightarrow \infty$ corresponds to the Ising model with $J_{z}=J \Delta$. The maximum of its magnetic susceptibility and specific heat is shifted, because of the infinite $\Delta$, towards infinite temperature $T / J$ and the height of its magnetic susceptibility approaches zero in units of $\chi \mathrm{J} / N$.

In Fig. 2.3 we show the magnetic susceptibility as a function of temperature for various choices of $\Delta$. With growing $\Delta$ the overall height of the susceptibility is lowered and shifted towards higher temperatures. This behavior is understandable, because for large $\Delta$ the potential contribution of the Hamiltonian becomes dominant, so that for very large $\Delta$ the rescaled susceptibilities of the different chains can be mapped onto that of the Ising model with the coupling constant $J \Delta$.

Note that in Fig. 2.3 temperature and magnetic susceptibility are presented in units 


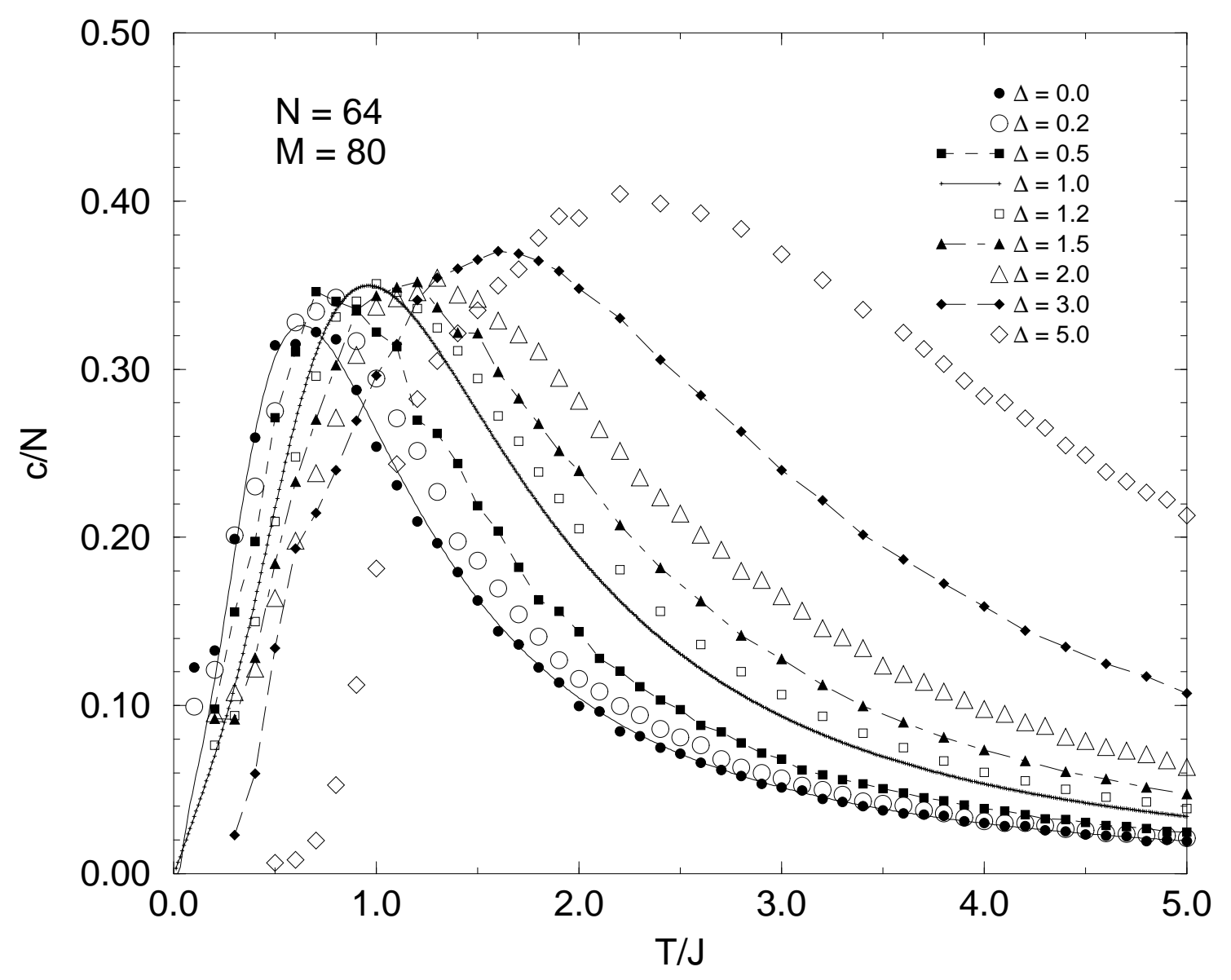

Figure 2.4: Specific heat per site versus temperature. The parameters are identical to those of Fig. 2.3. The anisotropy coupling constant $\Delta$ is chosen between 0.0 and 5.0. The data for $\Delta=1.0$ are taken from A. Klümper (isotropic model), $\Delta=0.0$ is the XX-model (the solid line shows the analytic data). A model with $\Delta \rightarrow \infty$ would correspond to the Ising model with infinite coupling constant $J \Delta$. The temperature of the maximum of its specific heat would be infinity. The chain length is $N=64$ and the Trotter number is $M=80$.

of $J$ and $1 / J$, respectively. Hence, the magnetic susceptibility of the Ising model which is nonzero in units of $1 /(J \Delta)$ is identical to zero for all temperatures in units of $1 / J$ if $\Delta \rightarrow \infty$.

In Fig. 2.4 we show the specific heat as a function of temperature for the same choices of $\Delta$. With growing $\Delta$ the overall height of the specific heat is lowered and shifted towards higher temperatures.

The loop algorithm allows the examination of systems of large size (e. g. $N=64$ and $M=80$ ). Because of the large chain length, finite-size effects are negligible for temperatures $T \geq 0.3 J$. 
The loop algorithm turned out to be a useful and fast algorithm for the investigation of pure Heisenberg models and Heisenberg models coupled to Einstein phonons. However, it includes a systematic error due to the finite Trotter number.

In the following section we will investigate the Heisenberg model with the continuous version of the loop algorithm as introduced by Beard and Wiese [81].

\subsection{Beard-Wiese algorithm}

In the continuous version of the loop algorithm the interaction plaquettes are replaced by segments in the direction of the Trotter time. These segments can be updated separately. Since the continuum limit is the limit $M \rightarrow \infty$, where $M$ is the Trotter number, this means that the diagonal plaquettes become horizontal "transition times" and the updates of the segments occur by means of a "decay constant" $\lambda=J$. By using this update procedure, new transition times are generated. For the XXX-model the new transition times can be generated only for segments whose two spins have opposite sign (named "optional decay" by Beard and Wiese [81]. The loops are created by following the spin-segments until a new transition times. Now the transition to the corresponding site has to occur and the loop proceeds in the opposite direction. Because of the zero-divergence criterion the loops always close and each spin-segment can be attributed to exactly one loop. For the XXX-model the loops can be flipped individually with the probability $1 / 2$. By their very nature, transition times separate regions with different spin. Due to the loop flip in average half of the former transition times will be eliminated. The configuration of spins and transition times generated by this update will be the start for the next update. An example is shown in Fig. 2.5.

The continuous loop algorithm has several advantages as compared to the discrete one.

(i) In contrast to the finite Trotter number case, there are no systematical errors.

(ii) Computer time is saved, because it is no longer required to update $M$ Trotter layers. Instead, segments with given continuous Trotter time interval are updated.

(iii) The correct number of transition times at given temperature results automatically from the update procedure. By contrast, in the discrete case the Trotter number has to be chosen by hand, so that it could either be too small (leading to systematical errors) or be unnecessarily large (requiring a lot of computer time).

We found that for given temperature and for given statistical convergence the computer time was ten times less for the continuous loop algorithm than for the discrete one.

To be complete, we have to note that an important advantage of the discrete loop algorithm has not got lost for the continuous version. The change of magnetization and change of winding number are automatically included in both the discrete and continuous loop algorithm.

In Figs. $2.6-2.8$ we show the magnetic susceptibility for the isotropic antiferromagnetic Heisenberg chain. With this fast algorithm we were able to calculate this quantity for chain lengths up to $N=256$ with very high precision, allowing the examination for temperatures down to $T=0.04 J$. 


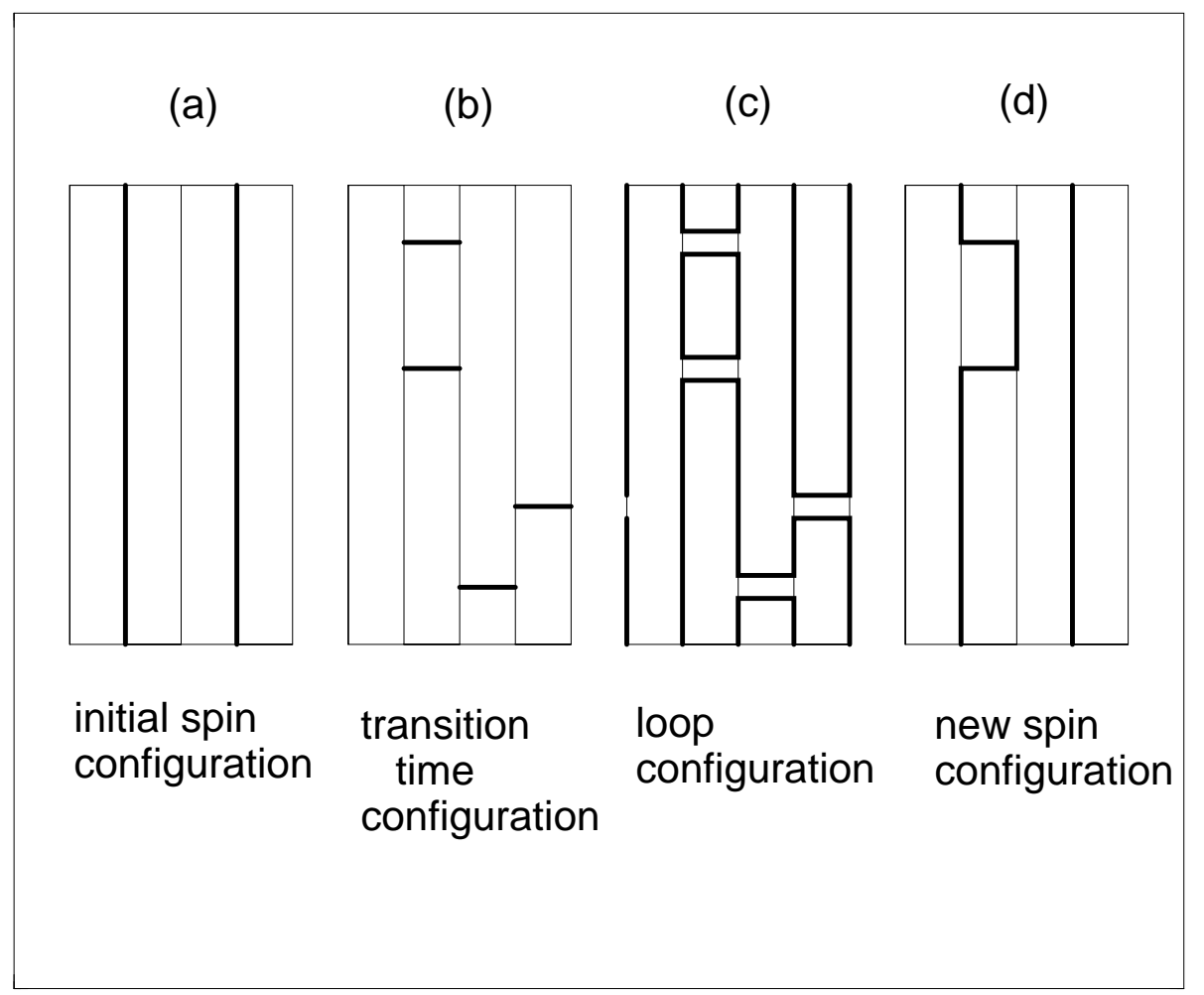

Figure 2.5: Update procedure of the continuous loop algorithm. We consider here a chain with $N=4$ lattice sites and periodic boundary conditions. The Trotter time is the ordinate. In this example, we start with an initial spin configuration with zero magnetization (a). We follow those bond-segments in the direction of the Trotter time which have unlike spins on their boundaries. In this example, we have four segments, where all satisfy this condition. With the help of the decay constant $\lambda$ (which is equal to the spin coupling constant $J$ for the XXX model) we find new transition times. These are denoted by horizontal bars in figure (b). In this example, we have four transition times. Loops are generated in the following way. Follow the spin-segments until a transition time is reached. Then switch to the neighboured spin-segment (whether the left or right neighbour is given by the transition). Follow this spin-segment until the next transition and continue until the loop closes. Then start with an other spin-segment which has not yet been attributed to a loop and construct a further loop. Continue until all spin-segments have been attributed to loops. Figure (c) shows a loop configuration consisting of two loops which have been generated by the transition time configuration (b). Now flip the loops with probability $1 / 2$. In this example, we flip the small loop, i. e. its spins will be flipped. By doing so we obtain the new spin configuration shown in figure (d). The transition from (a) to $(\mathrm{d})$ is called a spin-update.

By comparing our results with the exact ones of Klümper [19] we found very high precision: 


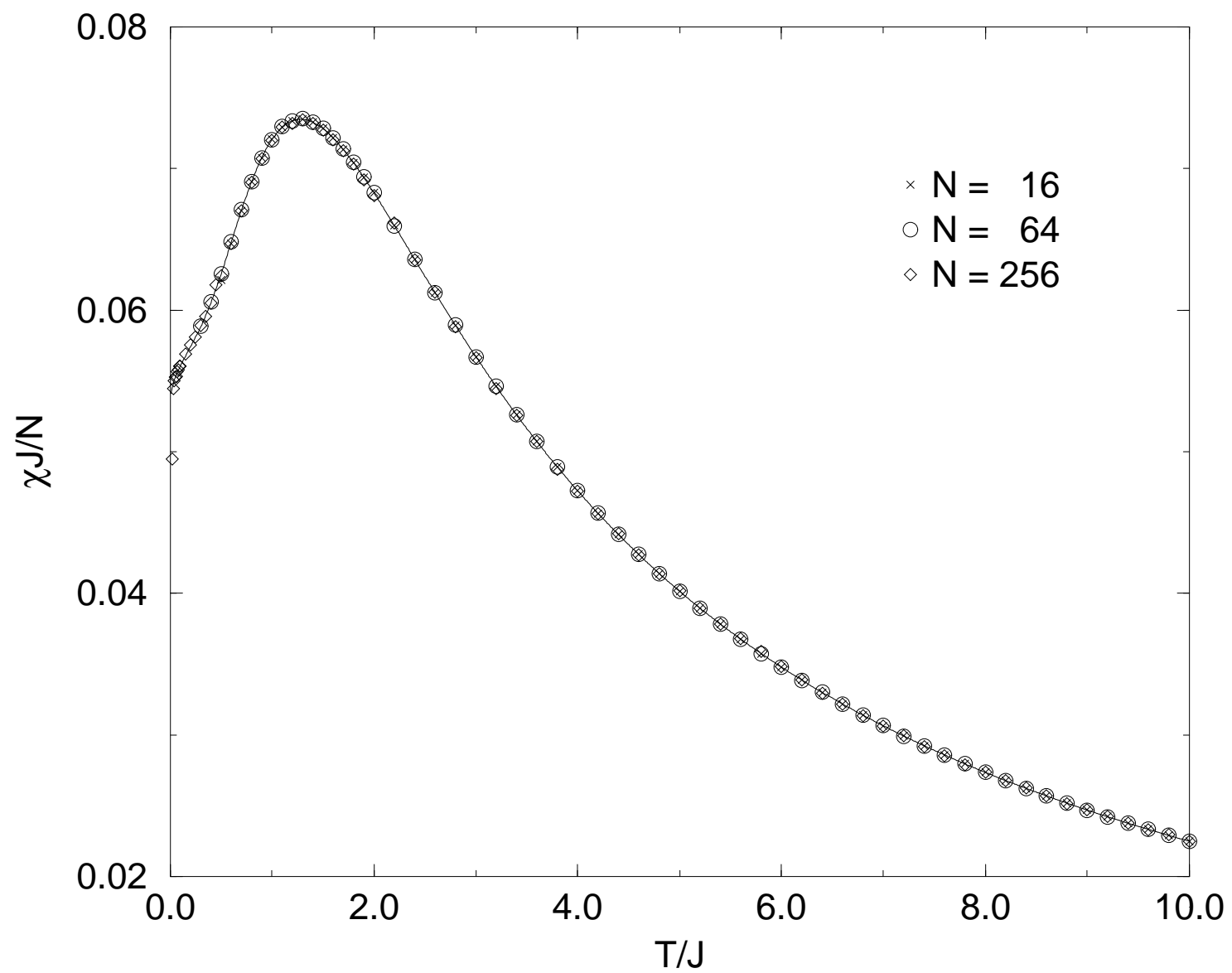

Figure 2.6: Magnetic susceptibility as a function of temperature for chain length $N=16$, $N=64$, and $N=256$, respectively. The number of spin-updates for each temperature is $10^{8}, 10^{7}$, and $10^{6}$, respectively. The precision is very high: $\Delta \chi J / N \leq 1 \times 10^{-4}$ and $\Delta \chi / \chi \leq 0.2 \%$ for $0.04 J \leq T \leq 0.9 J$ and $N=256 ; \Delta \chi J / N \leq 1 \times 10^{-5}$ and $\Delta \chi / \chi \leq$ $0.015 \%$ for $0.9 J \leq T \leq 10 J$ and $N=16$. The solid line shows the exact data from A. Klümper.

\begin{tabular}{|lcrc|}
\hline$\Delta \chi / \chi$ & $T / J$ & $N$ & number of updates \\
\hline$\leq 0.2 \%$ & $0.04, \ldots, 0.9$ & 256 & $10^{6}$ \\
$\leq 0.15 \%$ & $0.9, \ldots, 10$ & 16 & $10^{8}$ \\
\hline
\end{tabular}

\begin{tabular}{|lccc|}
\hline$\Delta c / c$ & $T / J$ & $N$ & number of updates \\
\hline$\leq 0.5 \%$ & $0.7, \ldots, 10$ & 64 & $10^{6}$ \\
\hline
\end{tabular}

\begin{tabular}{|lcrc|}
\hline$\Delta U / J$ & $T / J$ & $N$ & number of updates \\
\hline$\leq 1 \times 10^{-5}$ & $0.01, \ldots, 0.03$ & 1024 & $10^{5}$ \\
$\leq 4 \times 10^{-5}$ & $0.04, \ldots, 0.7$ & 256 & $10^{6}$ \\
$\leq 4 \times 10^{-5}$ & $0.7, \ldots, 10$ & 16 & $10^{8}$ \\
\hline
\end{tabular}




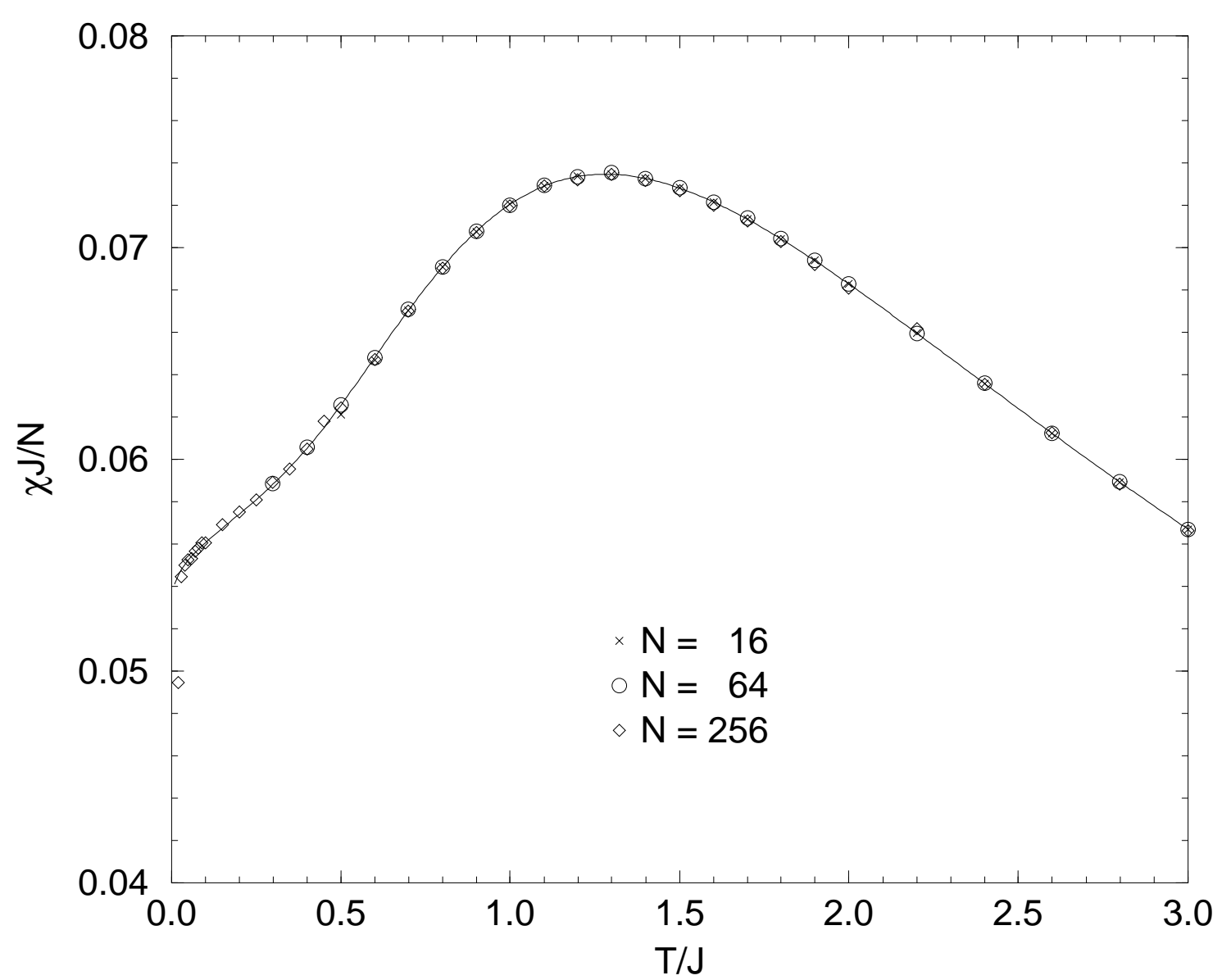

Figure 2.7: Magnetic susceptibility as a function of temperature for chain length $N=16$, $N=64$, and $N=256$, respectively. The number of spin-updates for each temperature is $10^{8}, 10^{7}$, and $10^{6}$, respectively. The precision is very high: $\Delta \chi J / N \leq 1 \times 10^{-4}$ and $\Delta \chi / \chi \leq 0.2 \%$ for $0.04 J \leq T \leq 0.9 J$ and $N=256 ; \Delta \chi J / N \leq 1 \times 10^{-5}$ and $\Delta \chi / \chi \leq$ $0.015 \%$ for $0.9 J \leq T \leq 10 J$ and $N=16$. The solid line shows the exact data from A. Klümper.

For chain length $N=64$ we were able to calculate the specific heat with high precision in the temperature range $0.15 J \leq T \leq 10 J$, see Figs. 2.9 and 2.10.

From the results above we can conclude on a very important advantage of the continuous loop algorithm in comparison to the discrete one. In both algorithms, the relative error resulting from finite size effects is constant over a large temperature range provided that the chain length $N$ is increased inversely proportional to the temperature $T$.

In the discrete loop algorithm the same is correct for the Trotter number $M$, i. e. the appropriate Trotter number to be chosen has to increase as $M \propto 1 / T$. The computer time for each update is proportional to $N \times M$. However, the precision of the quantum Monte Carlo simulation decreases if $N \times M$ is increased and the number of updates 


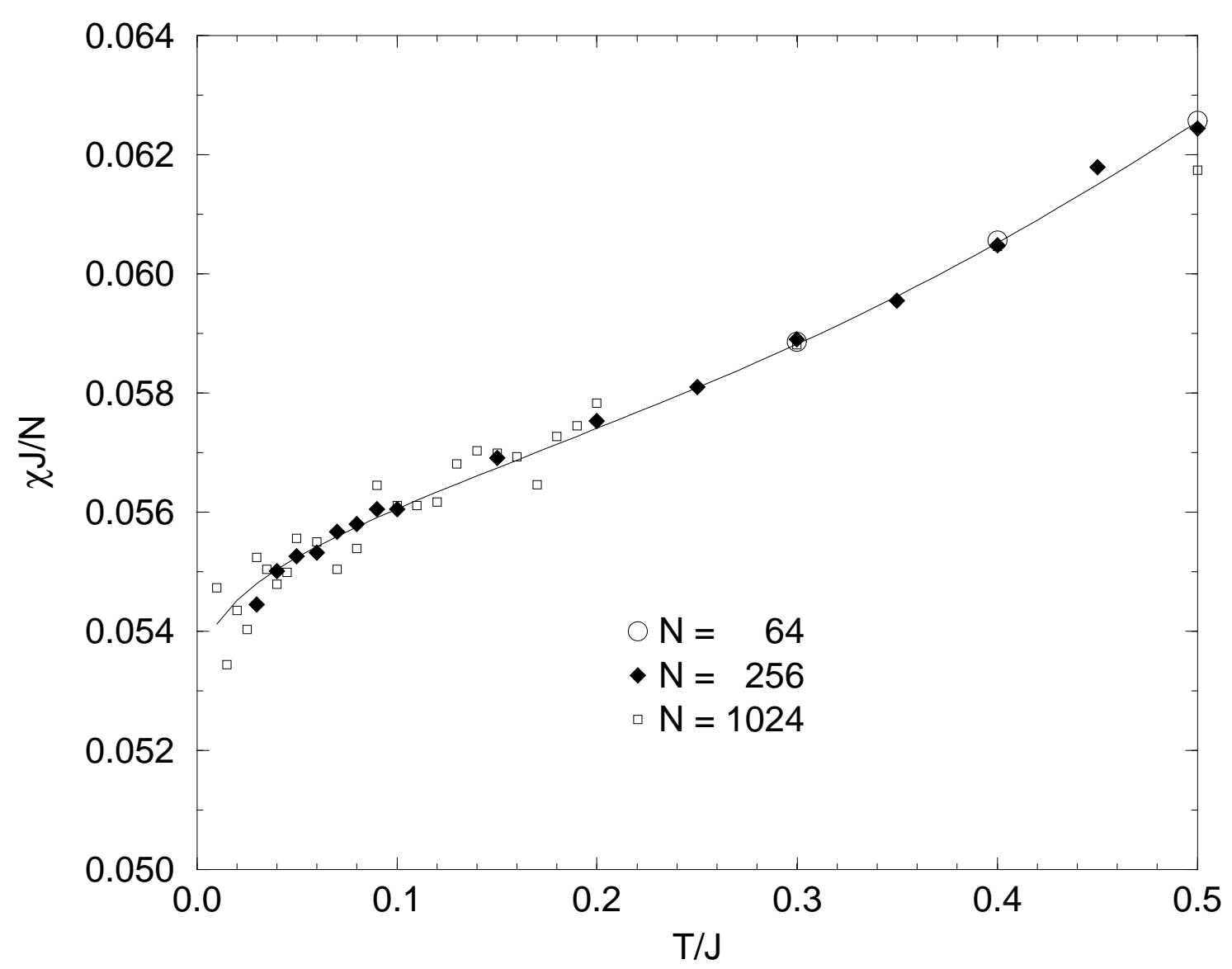

Figure 2.8: Low-temperature behavior of the magnetic susceptibility for chain length $N=64, N=256$, and $N=1024$, respectively. The number of spin-updates for each temperature is $10^{7}, 10^{6}$, and $10^{5}$, respectively. The curve for $N=1024$ is not smooth, because of insufficient statistics. The solid line shows the exact data from A. Klümper.

remains constant. To obtain the same precision for larger systems, one has to increase the number of updates. According to our experience, the number of updates has to be increased roughly as $N \times M$. Hence, for given precision, the investigation of a system requires a computer time which is proportional to $(N \times M)^{2}$ and therefore approximately proportional to $1 / T^{4}$.

In the continuous loop algorithm the number of transitions increases proportional to the inverse temperature (for $T \leq J$ ). The computer time for each update is proportional to the product of the chain length and the number of transition times. The difference between the discrete and the continuous loop algorithm is that in the continuous case the precision of the quantum Monte Carlo simulation increases if the chain length $N$ is increased and the number of updates remains constant. To obtain the same precision for larger systems, one has to decrease the number of updates. According to our experience, 


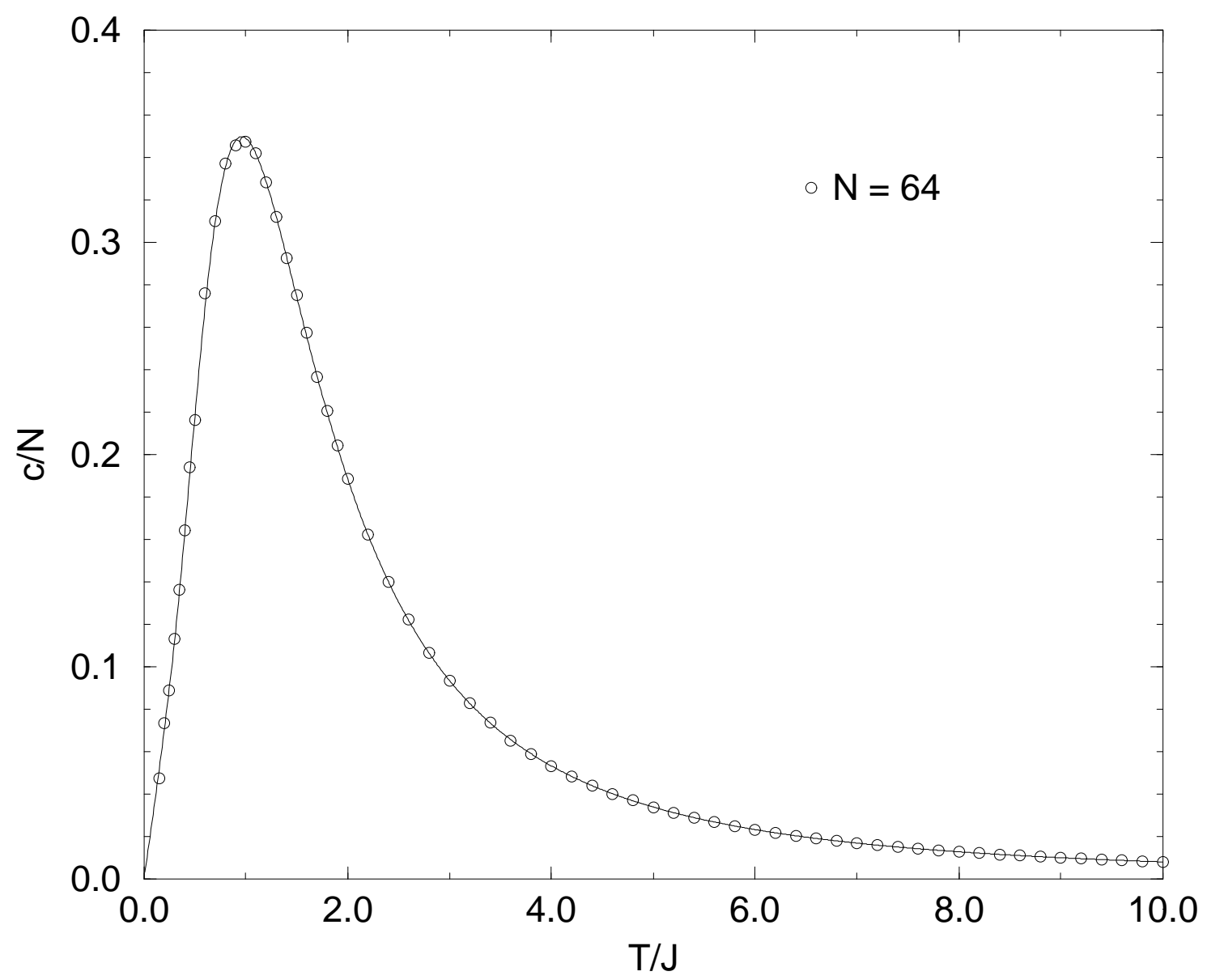

Figure 2.9: Specific heat per site versus temperature for a chain with $N=64$. The number of spin-updates for each temperature is $10^{6}$. The precision is $\Delta c / c \leq 0.5 \%$ for $0.7 J \leq T \leq 10 J$. The solid line shows the exact data from A. Klümper.

the number of updates has to be decreased roughly by $N^{2}$. Hence, for given precision, the investigation of a system requires a computer time which is nearly independent of the temperature. This property of the continuous loop algorithm saves much computer time with respect to the discrete one if systems at low temperatures are investigated. The gain increases roughly proportional to the fourth power of the inverse temperature.

This remarkable observation can be understood as follows. If the chosen temperature is not too low, then each update of a long chain is equivalent to several updates of a short chain, because very distant spins have decoupled. Unfortunately, for very low temperatures a large chain length is still required, because of the large spin-spin correlation length.

From the discrete case of the loop algorithm we can infer the mean number of diagonal plaquettes per site to increase linearly with the inverse temperature $\beta$ if the extrapolation 


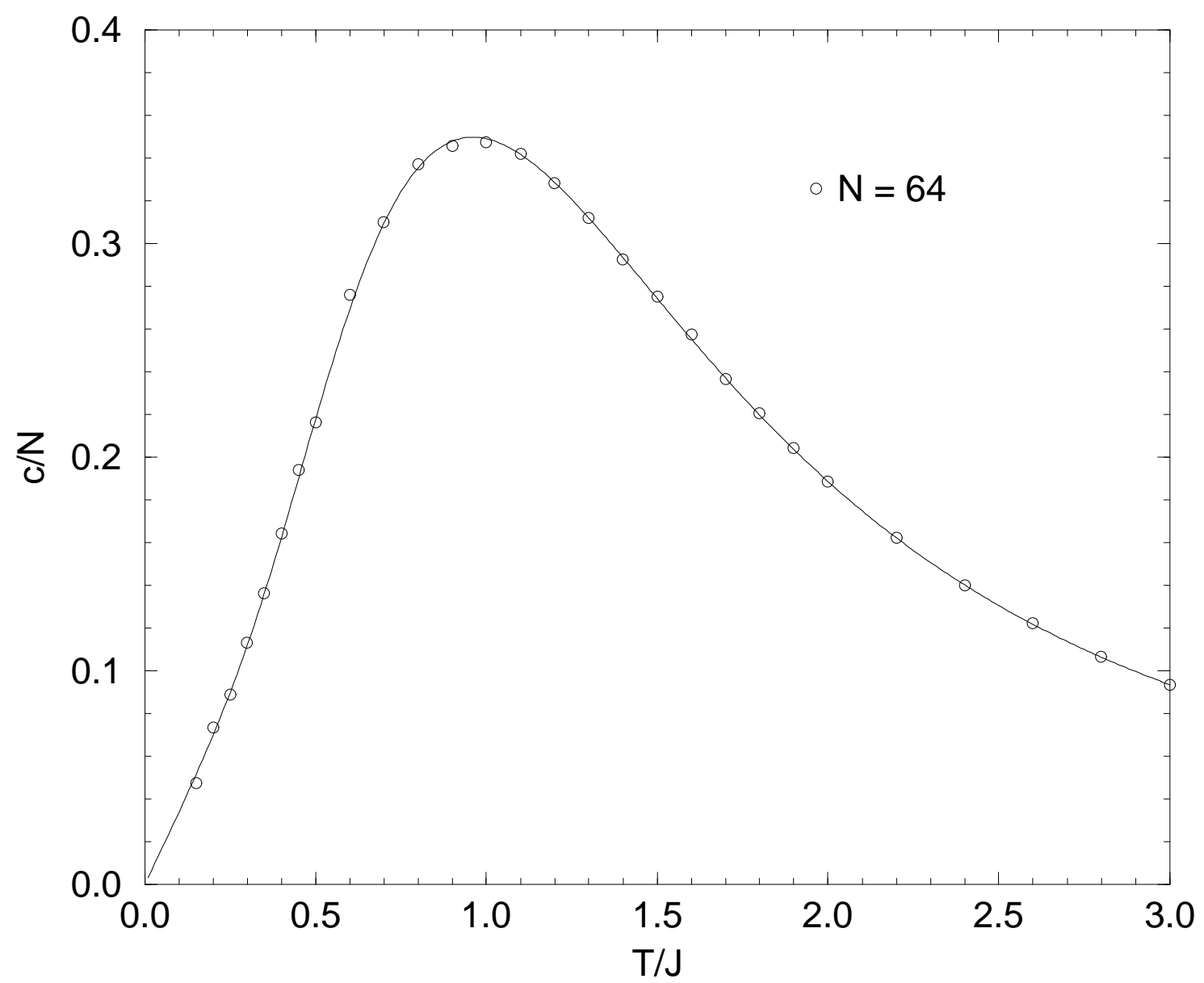

Figure 2.10: Like in Fig. 2.9 we present the specific heat per site versus temperature for a chain with $N=64$. The scale is enlarged in order to show the low temperature behavior and the peak more clearly. The data fit both the low temperature region and the peak with high precision. The solid line shows the exact data from A. Klümper.

to infinite Trotter number $M$ is performed. This can be understood as follows. The Boltzmann weights of the plaquettes of the discrete algorithm are given by Eq. (2.2). The mean number of diagonal plaquettes per site for infinite Trotter number is then approximately given by

$$
\lim _{M \rightarrow \infty}\left(M \frac{b}{a+b+c}\right)=\frac{\beta J}{2} .
$$

We calculated the mean number $N_{t t}$ of transition times per site. It should be identical to the number of diagonal plaquettes per site for infinite Trotter number in the discrete case. It is monotonously increasing with growing inverse temperature $\beta$. Its limiting value is

$$
\lim _{T \rightarrow 0} N_{t t}=1.181,79(11) \beta J
$$




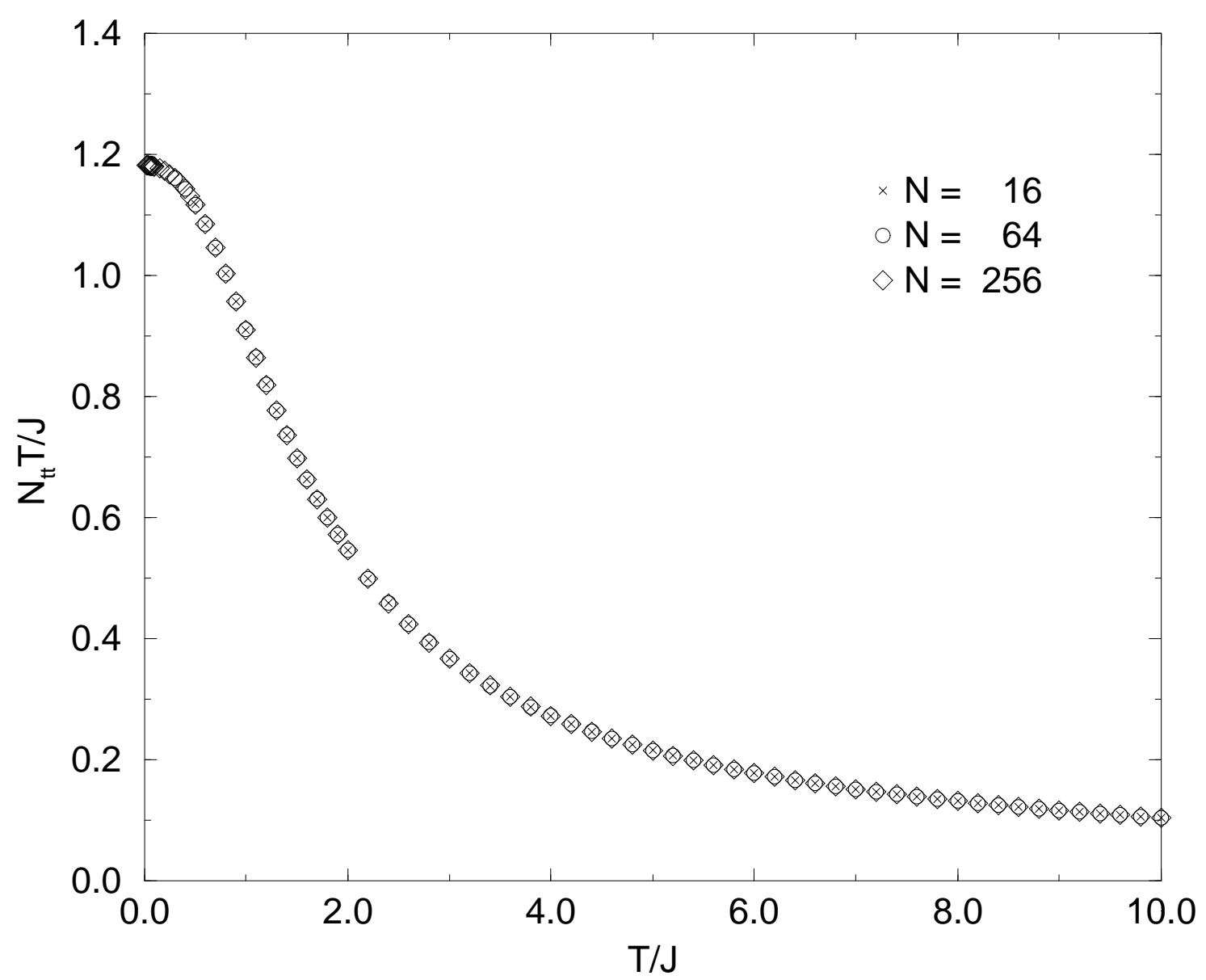

Figure 2.11: Mean number $N_{t t}$ of transition times per site multiplied with the temperature. The chain length is $N=16, N=64$, and $N=256$, respectively. One can infer from the figure that $N_{t t}<1$ for $T>5 \mathrm{~J}$. This means that in this high-temperature region the loops mainly follow the Trotter direction. The consequence for the discrete model is that a small Trotter number is sufficient to describe the model at high temperatures.

see Figs. 2.11 and 2.12 .

This equation justifies the claim that in the discrete case the required Trotter number $M$ is proportional to the inverse temperature. Furthermore, together with the behavior of the correlation length, this justifies to choose the Trotter number of the same order as the chain length.

\subsection{Dimerized model}

It is of phenomenological interest to study the dimerized model, i. e., the model with static dimerization $\delta$. This model can be described by a Hamiltonian similar to that of 


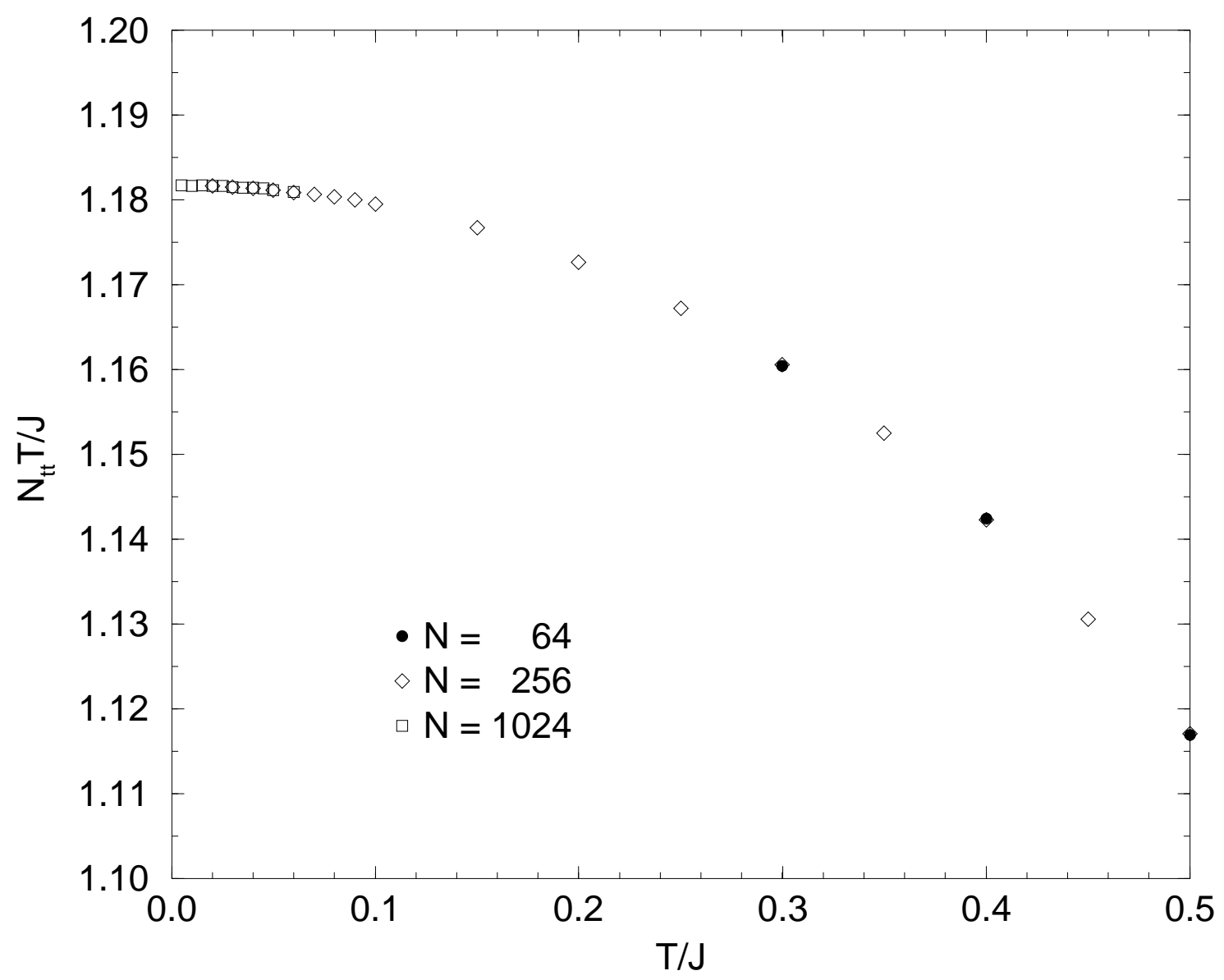

Figure 2.12: Mean number $N_{t t}$ of transition times per site multiplied with the temperature. The chain length is $N=64, N=256$, and $N=1024$, respectively. For $T \rightarrow 0$ we get $N_{t t} T / J \rightarrow 1.181,79(11)$. This proportional increase of the number of transition times with inverse temperature at small temperatures is the reason why we increase the Trotter number with the inverse temperature in later examinations.

the isotropic Heisenberg model, the only difference is the replacement of the spin-spin coupling constant $J$ by the alternating coupling $J_{e f f, l}=J\left(1+\delta(-1)^{l}\right)$,

$$
\mathcal{H}=\frac{J}{2} \sum_{l=1}^{N}\left(1+\delta(-1)^{l}\right) \vec{\sigma}_{l} \cdot \vec{\sigma}_{l+1} .
$$

The dimerized model is not exactly solvable by the Bethe ansatz. Therefore numerical methods are of particular importance for its study.

This model was the subject of intensive investigation [85-89]. It is the adiabatic limit of the spin-phonon model, Eq. (3.1), which will be examined in detail in the following chapters. The special case, Eq. (2.7), can be understood as a model with static dimerization which results from the consideration of only one phonon mode, i. e. one with fixed 


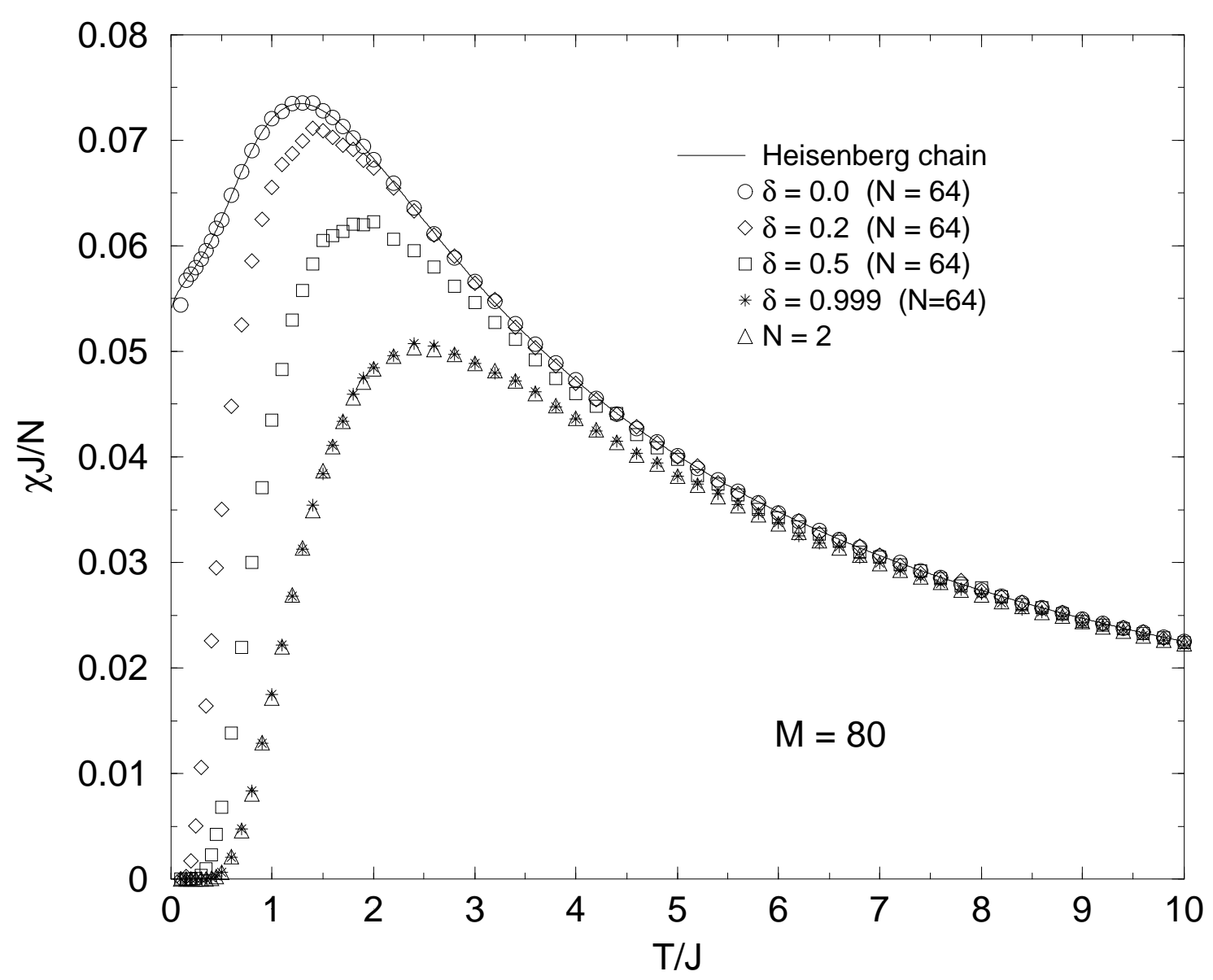

Figure 2.13: Magnetic susceptibility of a dimerized chain. The dimerization $\delta$ is varied between 0.2 and $0.999 . \delta=0$ corresponds to the undimerized model and $\delta=1$ to the completely dimerized model whose thermodynamic properties are identical with the $N=2$ chain which is shown for comparison. The exact data of the undimerized Heisenberg chain are from A. Klümper.

momentum $\pi / a$, with $a$ being the lattice constant.

The magnetic susceptibility for a chain with $N=64$ and $M=80$ as a function of temperature is shown for different choices of $\delta$ in Figs. 2.13 and 2.14. For comparison, the exact data for the undimerized Heisenberg chain and the numerical data for the $N=2$ chain are shown. The undimerized chain corresponds to the limit $\delta \rightarrow 0$ and the $N=2$ system corresponds to the limit $\delta \rightarrow 1$ (complete dimerization). The appearance of the spin gap is not a finite-size effect, but results from the dimerization.

As we will see in the discussion of spin-phonon systems, the static dimerization discussed in this chapter cannot be expressed in simple form as the dimerization at zero temperature generated by the spin-phonon coupling. Neither the magnetic susceptibilities nor the specific heats of the two models can be mapped onto one another. The magnetic 




Figure 2.14: Low-temperature region of the magnetic susceptibility of a dimerized chain. The dimerization $\delta$ is varied between 0.2 and $0.999 . \delta=0$ corresponds to the undimerized model and $\delta=1$ to the completely dimerized model whose thermodynamic properties are identical with the $N=2$ chain which is shown for comparison. The exact data of the undimerized Heisenberg chain are from A. Klümper.

susceptibility of spin-phonon systems (for not too low temperatures) can be mapped onto that of a Heisenberg system by the introduction of a global (temperature independent) effective spin-spin coupling constant. By contrast, no such effective spin-spin coupling can be found for static dimerized chains, where only at very high temperatures the magnetic susceptibility approaches that of the Heisenberg chain.

Furthermore, static dimerized chains cannot describe the physical spin-Peierls materials $\mathrm{CuGeO}_{3}$ and $\mathrm{NaV}_{2} \mathrm{O}_{5}$ correctly, because the magnetic susceptibility of these materials drops exponentially near the critical temperature from a finite value to zero, where above the temperature it can be described by a (frustrated) Heisenberg chain. This is in contrast to the static dimerized chain whose susceptibility approaches that of the Heisenberg 


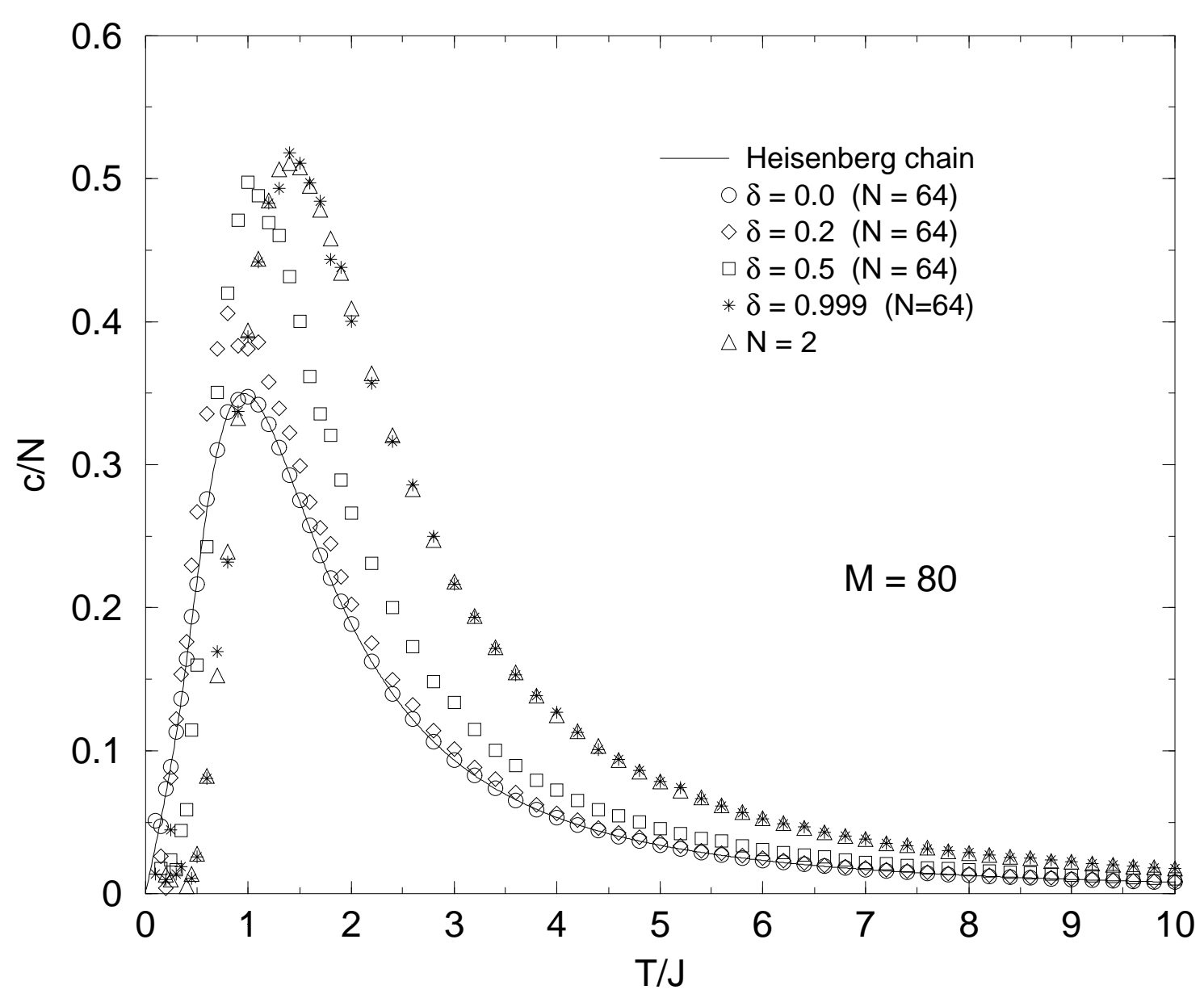

Figure 2.15: Specific heat of a dimerized chain. The dimerization $\delta$ is varied between 0.2 and 0.999. $\delta=0$ corresponds to the undimerized model and $\delta=1$ to the completely dimerized model whose thermodynamic properties are identical with the $N=2$ chain which is shown for comparison. The exact data of the undimerized Heisenberg chain are from A. Klümper.

chain only at very high temperatures. However, static dimerized chains might be useful for the description of these materials, if a nonzero dimerization is used below the critical temperature and a zero dimerization is used above the critical temperature.

For the same values of the dimerization $\delta$ we plot the specific heat as a function of temperature. The overall maximum occurs at higher temperatures and takes higher values for growing dimerization (Fig. 2.15).

Furthermore, we examine the correlation length $\xi$ as a function of the temperature $T$ and the dimerization $\delta$.

At zero temperature it is known that $\xi \propto \delta^{-2 / 3}[19]$. For the analysis of the data we will rescale both $\xi$ and $T$ with $\delta^{2 / 3}$. 


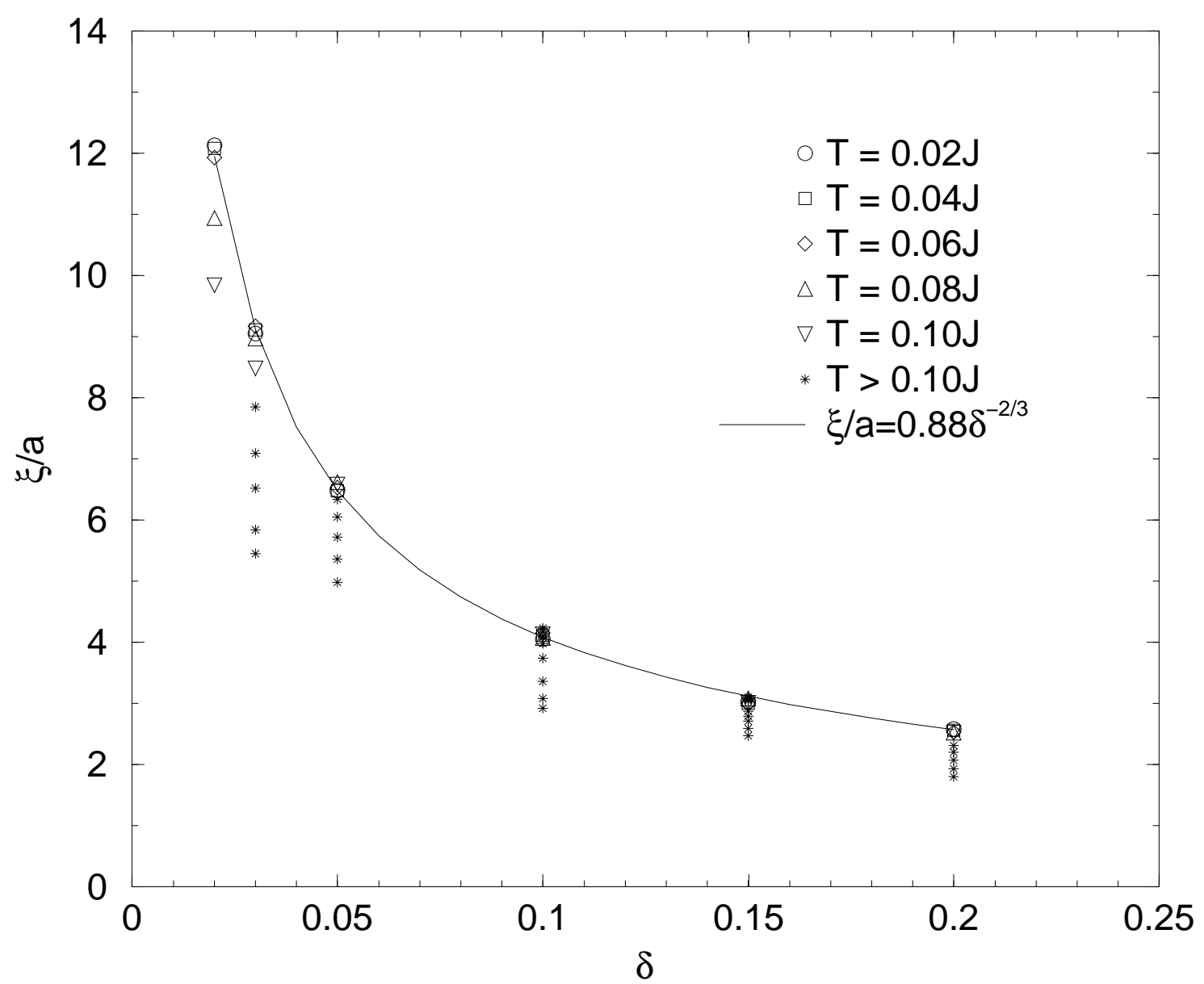

Figure 2.16: Spin-spin correlation length $\xi$ as a function of the dimerization $\delta$ for various temperatures. The chain length is $N=256$. The correlation lenghths were calculated with the Beard-Wiese algorithm and determined by a pure exponential fit. The correlation lengths at very low temperatures lie on the curve $\xi / a=0.88 \delta^{-2 / 3}$. The correlation lengths decrease for higher temperatures.

For $T=0$ and $\delta=0$ one expects a power law behavior of the correlation function, $\left\langle S_{l}^{z} S_{l+r}^{z}\right\rangle \propto 1 / r$. For finite and very low temperatures one expects

$$
\left\langle S_{i}^{z} S_{j}^{z}\right\rangle=\frac{(-1)^{|i-j|} e^{|i-j| a / \xi}}{|i-j|} .
$$

However, by fitting our data in this way we cannot confirm the scaling law for $\delta \neq 0$.

The fit gets even worse after rescaling both $\xi$ and $T$ by including logarithmic corrections, i. e. rescaling by $\delta^{2 / 3} / \sqrt{\ln |\delta|}$.

To solve the problem we will try a purely exponential fit of the correlation function, 


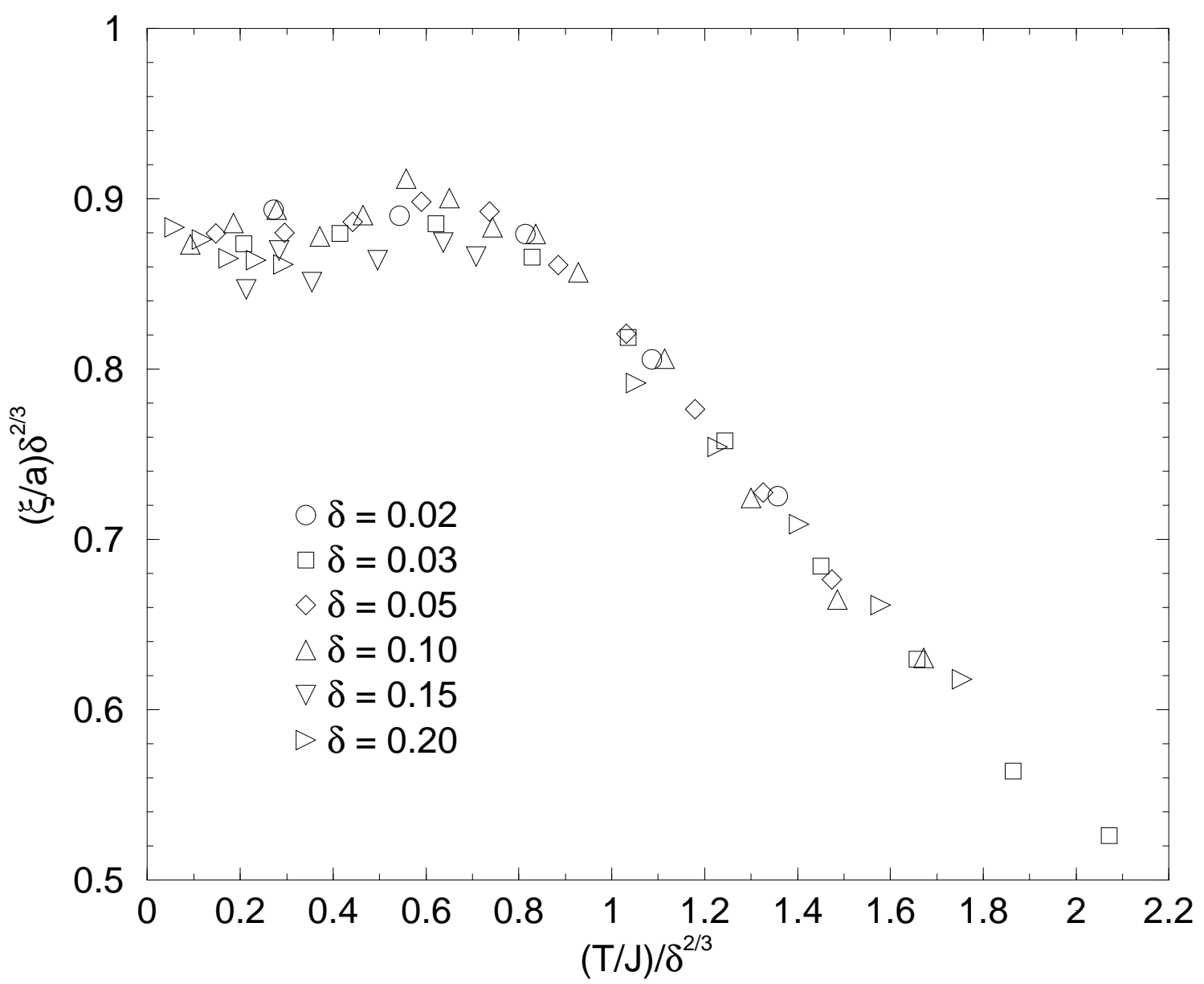

Figure 2.17: Spin-spin correlation length $\xi$ as a function of the temperature $T$ for systems with $N=256$ which were calculated with the Beard-Wiese algorithm. The correlation length was determined by a pure exponential fit. In this figure both $\xi$ and $T$ are rescaled with $\delta^{2 / 3}$, where $\delta$ is the dimerization.

i. e.

$$
\left\langle S_{i}^{z} S_{j}^{z}\right\rangle=(-1)^{|i-j|} e^{|i-j| a / \xi}
$$

We proceed by rescaling both $\xi$ and $T$ with $\delta^{2 / 3}$. By doing so it becomes obvious that the scaling law is satisfied at low temperatures and even at higher temperatures (see Fig. 2.17). As expected from dimensional considerations and known from previous work [90], also the spin gap $\Delta$ shows a scaling law, $\Delta \propto \delta^{2 / 3}$. More precisely,

$$
\begin{aligned}
\xi / a & =(0.88 \pm 0.01) \delta^{-2 / 3} \\
\Delta / J & =(0.7 \pm 0.1) \delta^{2 / 3} .
\end{aligned}
$$

The fit shows that $\xi$ becomes roughly temperature independent at very low temperatures and decreases with growing $\delta$. A check with the magnetic susceptibility data shows 
that this low temperature behavior is determined by the spin-gap. This means, within the spin gap, $\xi$ is roughly temperature independent and above the gap $\xi$ decreases with growing temperature.

To conclude, in the examined temperature region the spin-spin correlation can be fitted very well by an exponentially decaying function without power law contributions and logarithmic corrections. We cannot exclude that power law contributions may become significant at even lower temperatures than we have examined. 


\section{Chapter 3}

\section{Heisenberg chain coupled to Einstein phonons}

\subsection{The spin-Peierls transition in $\mathrm{CuGeO}_{3}$}

The discovery of the spin-Peierls phase transition in the inorganic compound $\mathrm{CuGeO}_{3}$ by Hase et al. [6] has motivated the examination of the one-dimensional Heisenberg model coupled to one-dimensional Einstein phonons. For this reason, I will review the properties of $\mathrm{CuGeO}_{3}$ in this section.

The crystal structure of $\mathrm{CuGeO}_{3}$ consists of octaeders and tetraeders with the oxygen atoms at the corners, a copper atom within the center of each octaeder and a germanium atom within the center of each tetraeder $[49,50]$.

The "unit cell" $a_{p} \times b_{p} \times c_{p}$ observed by Völlenkle et al. [49] turned out to be a pseudo unit cell. The actual unit cell is eight times larger, $a=2 a_{p}=9.5998 \AA, b=b_{p}=8.4665 \AA$, and $c=4 c_{p}=11.778 \AA$. This results from the behavior of the $\mathrm{GeO}_{4}$ tetraeders. A periodic change of their tilting and rotation is formed along the $c$-axis with the period of $4 c_{p}$. The directions of their rotations along the $a$-axis are in anti-phase. This results in a doubling of the pseudo unit cell along the $a$-axis [91].

The thermodynamic behavior of $\mathrm{CuGeO}_{3}$ can be described in good approximation by a one-dimensional isotropic antiferromagnetic spin-1/2 Heisenberg quantum spin system with nearest and next nearest neighbour interaction [46-48].

The spin-spin coupling constant of $\mathrm{CuGeO}_{3}$ was measured to be $J=1.7 \mathrm{THz}[92]$ or roughly $J \sim 100 K$.

The precise value of the frustration parameter for $\mathrm{CuGeO}_{3}$ is under discussion. Recent studies yielded $\alpha \simeq 0.24$ [46], $\alpha \simeq 0.354$ [48], and $\alpha \simeq 0.36$ [47].

The spin-Peierls phase transition is generated mainly by phonons of two frequencies $[51,52,93]$. The values of the angular frequencies $\Omega$ and the spin-phonon couplings $g$ are not precisely known. Braden et al. [51] measured $\Omega_{1}=3.2 \mathrm{THz}$ and $\Omega_{2}=6.8 \mathrm{THz}$. Gros and Werner [93] found $\Omega_{1}=3.13 \mathrm{THz}=151 \mathrm{~K}, \Omega_{2}=6.65 \mathrm{THz}=317 \mathrm{~K}, g_{1} J / 2=$ $0.45 \mathrm{THz}$, and $g_{2} \simeq 4 g_{1}$. In a later publication, Werner et al. [52] found $\Omega_{1}=3.12 \mathrm{THz}$, $\Omega_{2}=6.53 \mathrm{THz}, g_{1} J / 2=-15 \mathrm{~K}$, and $g_{2} J / 2=58 \mathrm{~K}$. To conclude, we get the approximate values $\Omega_{1}=2 J$ and $\Omega_{2}=4 J$ for the angular frequencies and $g_{1}=-3 / 8$ and $g_{2}=3 / 2$ for 
the spin-phonon coupling constants.

X-ray diffuse scattering measurements in $\mathrm{CuGeO}_{3}$ in the temperature range $14 \mathrm{~K} \leq$ $T \leq 40 \mathrm{~K}$ have shown that the inverse correlation length in the $c$-direction behaves as

$\sqrt{T-T_{s p}}$, where $T_{s p}=14 \mathrm{~K}$ is the critical temperature of the spin-Peierls phase transition [94]. The corresponding (non-classical) critical exponent is $\nu=1 / 2$.

\subsection{Introduction of the Heisenberg model coupled to phonons}

Pytte [42] and Cross and Fisher [43] have presented one-dimensional spin models in which the spin-Peierls phase transition is generated by three-dimensional phonons. These models are at present too difficult to be examined by numerical methods.

This is why one-dimensional spin models coupled to dispersionless one-dimensional phonons have been investigated. Because of the Mermin-Wagner theorem [10] these models cannot generate a spin-Peierls transition at finite temperatures.

The Hamiltonian we examine is,

$$
\mathcal{H}=\frac{J}{2} \sum_{l=1}^{N}\left(\vec{\sigma}_{l} \vec{\sigma}_{l+1}-t\right)\left(1+g\left(b_{l}^{\dagger}+b_{l}\right)\right)+\Omega \sum_{l=1}^{N} b_{l}^{\dagger} b_{l},
$$

where $b^{\dagger}$ and $b$ denote the phonon creation and annihilation operators, $\vec{\sigma}$ is the Pauli matrix, $g$ is the spin-phonon coupling, and $\Omega$ is the phonon frequency. The shift by $t$ in $\vec{\sigma}_{l} \vec{\sigma}_{l+1}-t$ is required for quantum Monte Carlo studies, because the minus sign problem appears whenever $t<1$. The value $t=1$ was used by Pytte [42] in his pioneering work on spin-Peierls systems. The same shift has been used also in later investigations [7, 95, 96].

For this model, Sandvik and Campbell [9] have shown the existence of a phase transition from the undimerized to the dimerized phase if the spin-phonon coupling $g$ exceeded a critical value $g_{c}(\Omega)$. Phase diagrams for a wide range of parameters have been presented by Weiße et al. [97] and Kühne and Löw [98], in final form by Raas and Uhrig [99].

Sandvik et al. [7] investigated the model via quantum Monte Carlo for a special choice of parameters: $\Omega=0.2 J$ and $g=1 /(4 \sqrt{2})$. They measured the magnetic susceptibility and the effective spin-spin coupling as a function of the temperature. They found the system to be in the dimerized phase, in accordance with the later phase diagram of Raas and Uhrig [99].

Augier et al. [95] examined this model with an exact diagonalization calculation by using the coherent states introduced by Fehrenbacher [100, 101]. For a four site chain with $\Omega=2 J$ they found that the mean phonon occupation number increases with $g$.

\subsection{Approximations of the model}

The investigation of a spin-phonon system requires a number of approximations which have to be kept in mind when one attempts to compare the theoretical results of the model 
with experimental observations of real substances. These approximations are necessary because finite computer time and conceptual problems such as the minus sign problem and difficulties to find an appropriate algorithm requires the restriction on a finite (often very small) number of degrees of freedom. The approximations of our model include:

- The system is restricted to the one-dimensional case, i. e. we consider a chain. This is opposed to the well known observation that physical solids are three dimensional systems. However, one-dimensional models can describe with good approximation the magnetic properties of three-dimensional materials whose magnetic structure is quasi one-dimensional. This means the interchain couplings have to be weak compared to intrachain couplings. Spin-Peierls materials are an example.

- The spin system is restricted to the isotropic case (i. e. the XXX model). Anisotropic contributions (i. e. the more general XXZ or XYZ model) are not considered.

- The spin interaction is restricted to the nearest neighbour interaction. Longer-range interactions (next-nearest neighbour etc.) have been neglected. This restriction was necessary because of the appearance of the minus sign problem in these models which leads to negative probabilities. Physical systems, however, sometimes do show longer range interactions. Only very few materials such as $\mathrm{NaV}_{2} \mathrm{O}_{5}$ can be described by restriction to nearest neighbour interactions.

- The investigated system is restricted to one-dimensional phonons. Actually, phonons are three dimensional entities. The spin-Peierls transition requires three dimensional phonons $[42,43]$.

- We restrict ourselves to dispersionless phonons. This is evidently an oversimplification, because phonons of real materials do always show dispersion. However, the Peierls-active phonon modes of $\mathrm{CuGeO}_{3}$ have rather sharp angular frequencies, $\Omega_{1}=2 J$ and $\Omega_{2}=4 J[52]$.

- The ladder operators of the dispersionless phonons are localized. We restrict our study to bond phonons. This approach was used also in previous investigations [7, 9, $88,95,96,98]$. However, if the phonons are localized at the spin sites then the spinphonon coupling can generate an effective frustration $[8,102]$. Naturally, a complete understanding has to include both bond and site phonons. Recent observations on $\mathrm{CuGeO}_{3}$ appear to show a contribution of spin-phonon coupling to the frustration [92].

- The system is restricted to the harmonic approximation. We do not consider interactions of higher order such as phonon self-interaction. This approximation is justified, because spin-Peierls phase transitions are assumed to be generated by spin-phonon interactions $[42,43]$.

- The phonon frequency is assumed to be temperature independent. This is not necessarily the case for physical systems where a softening of the phonon (i. e. a 
decrement of its frequency with decreasing temperature) may occur near the phase transition.

- The spin-phonon coupling is assumed to be temperature independent. This is not necessarily the case for physical systems. However, the experimental situation cannot yet decide whether the spin-phonon coupling is temperature independent or not.

- We are restricted to the monoatomic lattice. Phononic contributions due to the structure of lattice cells are not included in our model. This approximation, however, is most probably justified, because structural changes such as the spin-Peierls phenomenon should be valid for a large class of materials but not depend on their crystal structure.

\subsection{The modified loop algorithm}

The simulation of a Heisenberg chain coupled to phonons requires a modification of the loop algorithm. Only the spins can be updated globally. The phonons, however, have to be updated locally, because there is no conservation of phonon number. Hence, spins and phonons have to be updated separately and alternatingly. Since the local phonon update yields larger autocorrelation times, a quantum Monte Carlo step consists of one spin update and $n$ phonon updates.

We will consider an isotropic antiferromagnetic Heisenberg chain coupled to (dispersionless) Einstein phonons. The Hamiltonian used is Eq. (3.1) with $t=1$.

By using the Baker-Campbell-Haussdorff equation we can describe the contributions of the spin-spin and the spin-phonon interaction simultaneously, although the phonon generator $b^{\dagger}$ does not commute with the phonon annihilator $b$. The partition function $Z$ can therefore be written as a product of expectation values whose operators are blockdiagonalized in the spin space into $2 \times 2$ blocks and diagonalized in the phonon space. The interaction part of the Hamiltonian can therefore be described by two Trotter layers. A third Trotter layer is required for the description of the free phonons.

The partition function of the examined model can be written as,

$$
\begin{aligned}
Z= & \lim _{M \rightarrow \infty} \sum_{k_{0}, \ldots, k_{3 M-1}} \prod_{j=0}^{M-1} \\
& \left(\prod_{l=0}^{N-1}\left\langle k_{3 j, l}\left|\mathrm{e}^{-\beta \Omega\left(b_{l}^{\dagger} b_{l}\right) / M}\right| k_{3 j+1, l}\right\rangle\right) \\
& \left(\prod_{l=0}^{N / 2-1}\left\langle k_{3 j+1,2 l}\left|\mathrm{e}^{-\beta \mathcal{H}_{2 l} / M}\right| k_{3 j+2,2 l}\right\rangle\right. \\
& \left.\left\langle k_{3 j+2,2 l+1}\left|\mathrm{e}^{-\beta \mathcal{H}_{2 l+1} / M}\right| k_{3 j+3,2 l+1}\right\rangle\right),
\end{aligned}
$$

where $k$ denotes the spin and phonon configurations of the lower and upper edge of the interacting plaquettes, the inverse temperature is $\beta$, the Trotter number $M$, and the 
Hamiltonian for the spin-spin and the spin-phonon interaction is

$$
\mathcal{H}_{l}=\frac{J}{2}\left(\vec{\sigma}_{l} \vec{\sigma}_{l+1}-1\right)\left(1+g\left(b_{l}^{\dagger}+b_{l}\right)\right) .
$$

The Boltzmann weights for the local interaction part are,

$$
\begin{aligned}
\left\langle++m\left|\exp \left(-\beta \mathcal{H}_{l} / M\right)\right|++n\right\rangle= & \delta_{m, n} \\
\left\langle+-m\left|\exp \left(-\beta \mathcal{H}_{l} / M\right)\right|+-n\right\rangle= & \frac{1}{2} \delta_{m, n}+\frac{1}{2} \sqrt{m ! n !} \exp 2\left(\frac{\beta J}{M}+\left(\frac{\beta J g}{M}\right)^{2}\right) \\
\left\langle+-m\left|\exp \left(-\beta \mathcal{H}_{l} / M\right)\right|-+n\right\rangle= & \frac{1}{2} \delta_{m, n}-\frac{1}{2} \sqrt{m ! n !} \exp 2\left(\frac{\beta J}{M}+\left(\frac{\beta J g}{M}\right)^{2}\right) \\
& \left.\sum_{k=\max \{0, m-n\}}\right)^{2 k+n-m} \frac{1}{k !(m-k) !(k+n-m) !} \\
& \sum_{k=\max \{0, m-n\}}^{m}\left(\frac{2 \beta J g}{M}\right)^{2 k+n-m} \frac{1}{k !(m-k) !(k+n-m) !},
\end{aligned}
$$

where $m$ and $n$ denote the phonon occupation number at the lower and upper edge of the plaquette, respectively. Spin up and spin down are denoted by + and - .

Both spins and phonons are updated with a local heat bath update. It is obvious that detailed balance is satisfied for the two steps and therefore also for the whole procedure.

According to our experience, by using the importance sampling technique, $n=20$ phonon updates per spin update is an appropriate choice for a wide range of parameters. For the evaluation of the expectation values only the last of the phonon updates will be used. Moreover the first $10 \%$ of the quantum Monte Carlo steps will be used for thermalization only.

In our simulations we examine systems with chain lengths $N=16, \ldots, 256$ and Trotter number $M=20, \ldots, 320$, where $N=64$ and $M=80$ are typical values. The phonon frequencies are chosen between $\Omega=0.2 J$ and $\Omega=20 J$, the spin-phonon coupling are varied between $g=0$ and $g=1.5$.

We consider typically $10^{5}, \ldots, 10^{6}$ configurations for the spin updates (and therefore $2 \cdot 10^{6}, \ldots, 2 \cdot 10^{7}$ phonon sweeps) per temperature value.

\subsection{Effects of finite size and finite Trotter number}

Suzuki [103] found the partition function for finite Trotter number $M$ to have contributions which are proportional to $1 / M^{2}$. As a consequence, all expectation values have contributions proportional to $1 / M^{2}$ [104]. This was shown by Marcu et al. for the magnetic susceptibility, the internal energy, and the specific heat [68, 104, 105]. However, a complication appears, because the expectation values have further contributions which 


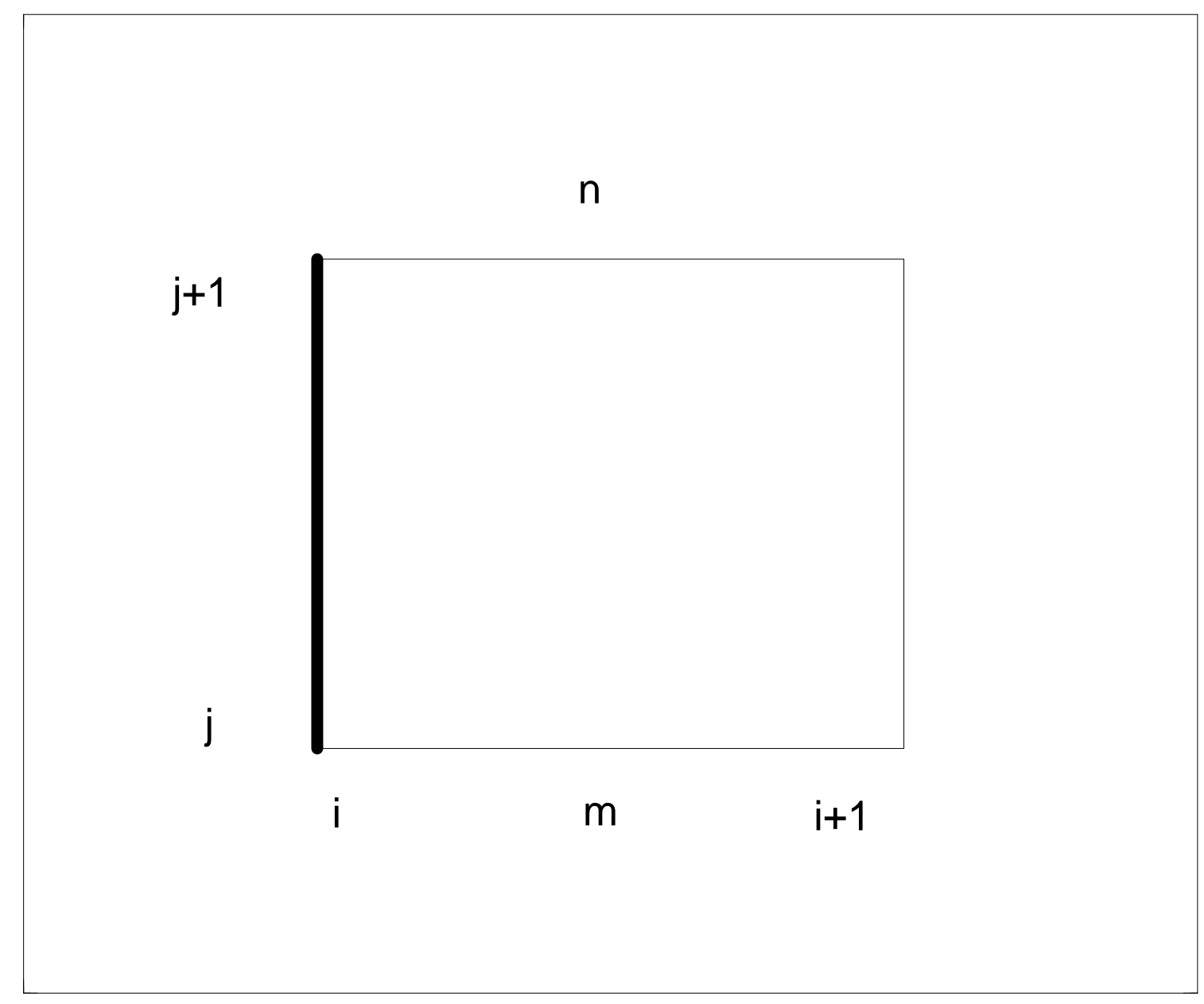

Figure 3.1: Interaction plaquette for the spin-phonon loop algorithm. The sites of the spins are denote by $i$ and $i+1$. The Trotter layers are denoted by $j$ and $j+1$. The phonon occupation of the (horizontal) bonds are denoted by $m$ and $n$. Naturally, for this plaquette there exists also a counterpart where up- and down-spins are interchanged.

are proportional to $1 / M^{n}$, where $n$ is an even integer. Hence, an extrapolation to the infinite Trotter number case which considers only the contributions which are proportional to $1 / M^{2}$ is not necessarily sufficient.

In the following, we will show under which conditions an extrapolation is sufficient which considers only the contributions proportional to $1 / M^{2}$. In some cases, however, one has to consider the contributions proportional to $1 / M^{4}$.

Let us first investigate the high-temperature region where small Trotter numbers should be sufficient. We plot the magnetic susceptibility (Fig. 3.2) and the internal energy (Fig. 3.3) versus $1 / M^{2}$ for a short chain with $N=16$ sites (with $g=0.2$ and $\Omega=J$ ) and $M=10, \ldots, 80$. For $T=0.2 J$ we confirm the dependence of the thermodynamic properties on $1 / M^{2}$. For larger temperatures, $T \geq 0.5 J$ the statistical fluctuations are 


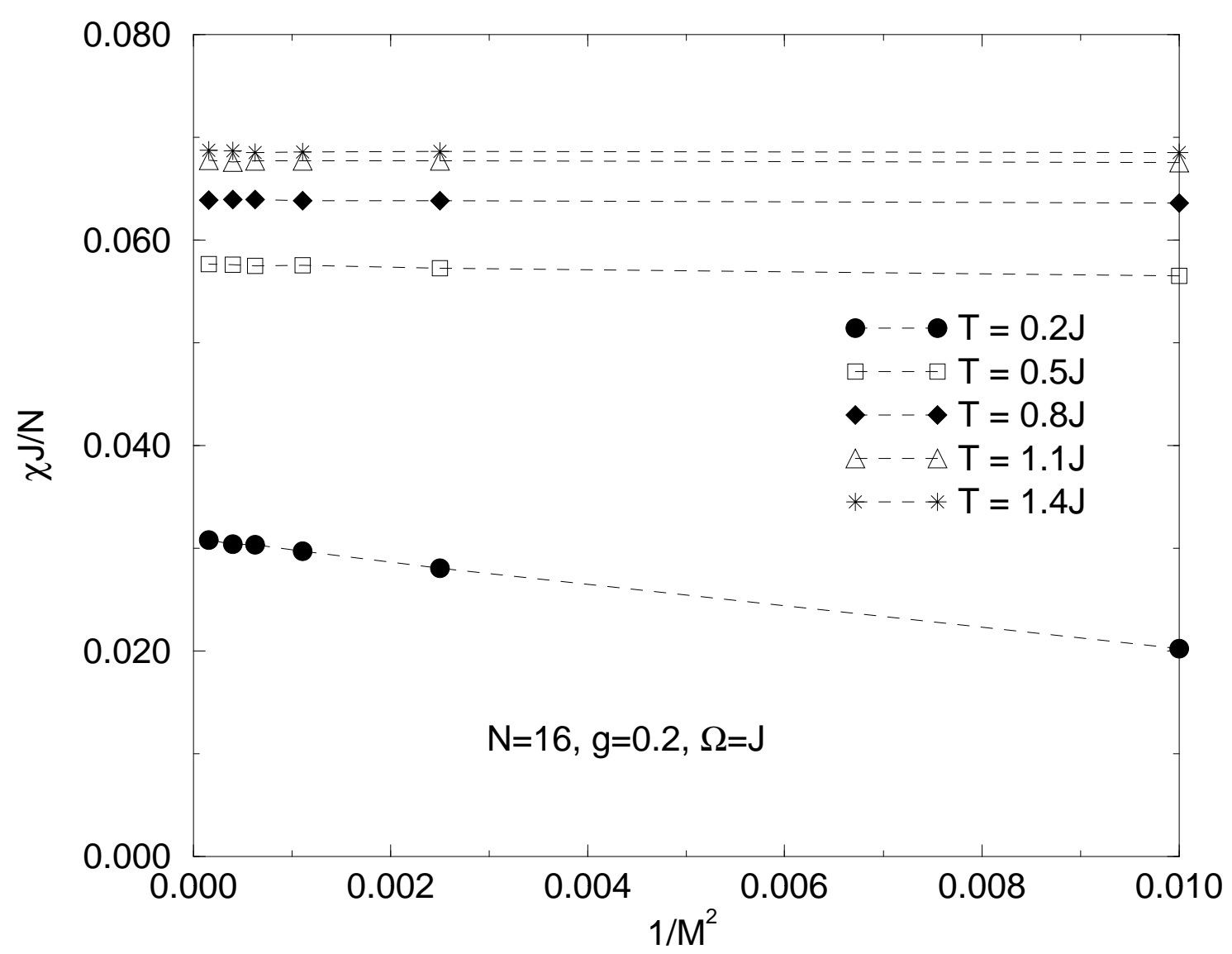

Figure 3.2: Magnetic susceptibility as a function of the inverse of the square of the Trotter number. The chain length is $N=16$, the spin-phonon coupling constant is $g=0.2$, and the phonon frequency is $\Omega=J$. The temperature is varied in the range $T=0.2 J, \ldots, 1.4 J$.

larger than the contributions of the finite Trotter number effects. Hence, if not very high accuracy is required, then for $T \geq 0.5 J$ a Trotter number as small as $M=20$ is sufficient. This result is important to save computer time which increases linearly with $M$.

Let us now regard the low-temperature region. Here, it is no longer sufficient to use small Trotter numbers.

For the Heisenberg model we chose the chain length $N=64$ and examined the system at the fixed temperature $T=0.2 J$. The spin-spin correlation depends clearly on the inverse Trotter number squared (Fig. 3.4). This is correct for all distances of the sites, i. e. $|i-j|=1, \ldots, N / 2$. Also the internal energy, specific heat, and magnetic susceptibility show the $1 / M^{2}$ proportionality. The result for infinite Trotter number can be obtained by extrapolation with the $1 / M^{2}$ law (Fig. 3.5).

From the same figure we see that the proportionality constant of the $1 / M^{2}$ contribution is completely different for the different physical properties. The data for $M=20$ differ from those for $M=\infty$ by only $1 \%$ for the magnetic susceptibility, by $3 \%$ for the internal 


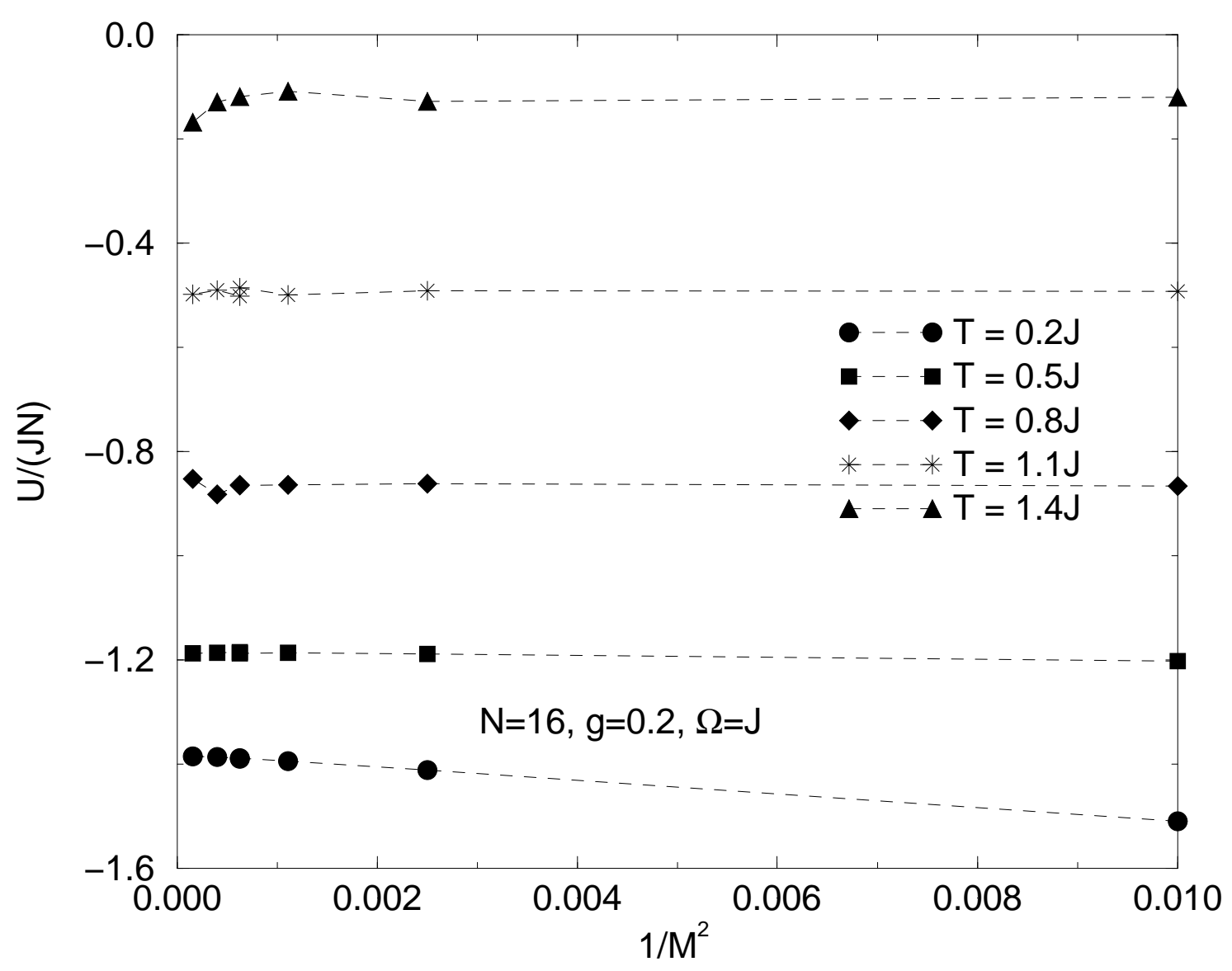

Figure 3.3: Internal energy as a function of the inverse of the square of the Trotter number. The chain length is $N=16$, the spin-phonon coupling constant is $g=0.2$, and the phonon frequency is $\Omega=J$. The temperature is varied in the range $T=0.2 J, \ldots, 1.4 J$.

energy, and by $200 \%$ for the specific heat.

In the following four figures we present the results for a chain with $g=1.0$ and $\Omega=2 J$ at fixed temperature $T=0.2 \mathrm{~J}$. The dependence of the staggered spin-spin correlation on $M$ is shown for the chain length $N=32$ (Fig. 3.6) and for $N=64$ (Fig. 3.7). For distances $|i-j| \leq N / 5$ we find only terms proportional to $1 / M^{2}$. For larger distances, $1 / M^{4}$ corrections have also to be considered.

The mean phonon occupation number (Figs. 3.8a and 3.9a), the effective spin-spin coupling constant $J_{\text {eff }}(T)$ (Figs. 3.8b and 3.9b), and the internal energy (Figs. 3.8d and $3.9 \mathrm{~d}$ ) show the linear dependence on $1 / M^{2}$, i. e. the value for infinite Trotter number can easily be extrapolated. For the magnetic susceptibility the $1 / M^{4}$ contributions have to be considered (Fig. 3.9c).

The data (for the system with $\Omega=2 J, g=1.0$, and $T=0.2 J$ ) for $M=20$ differ from those for $M \rightarrow \infty$ in the range $10 \%$ to $40 \%$ for the different observables. The 

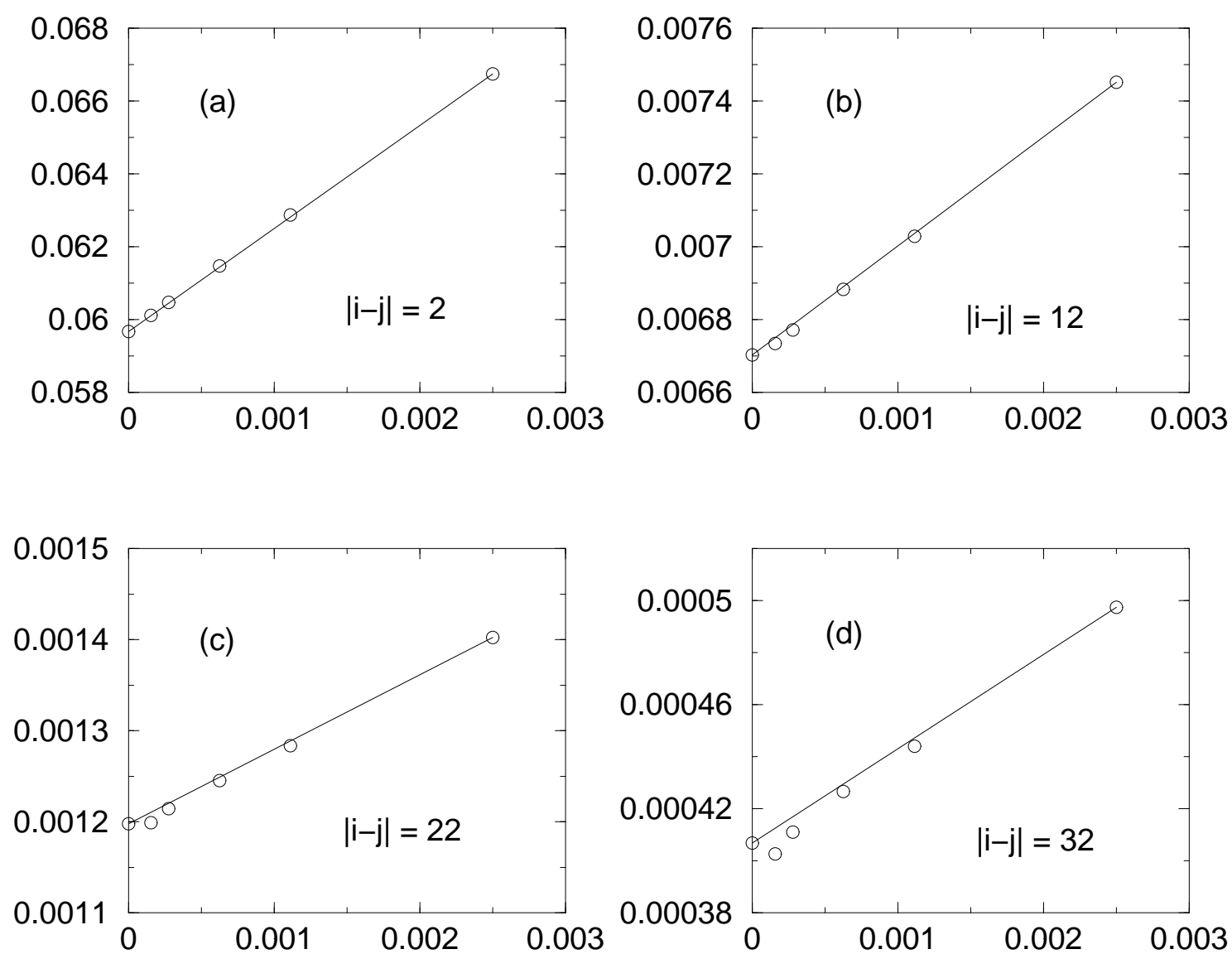

Figure 3.4: Spin-spin correlation $\left\langle S_{i}^{z} S_{j}^{z}\right\rangle$ of the Heisenberg model as a function of the inverse Trotter number squared. The temperature is $T=0.2 J$, the number of spinupdates is $10^{6}$, the chain length is $N=64$, and the examined Trotter numbers are $M=20,30,40,60,80, \infty$. We show the results for mutual distances $|i-j|$ of the sites of $2,12,22$, and 32 , respectively.

contribution of the $1 / M^{2}$ term is $14 \%$ for the mean phonon occupation number, $10 \%$ for the effective spin-spin coupling constant, $16 \%$ for the internal energy, and $20 \%(N=64)$ or $40 \%(N=32)$, respectively, for the magnetic susceptibility (Figs. 3.8 and 3.9).

In the present work we either extrapolated our results in the Trotter number, according to the law above, or when we found the statistical fluctuations to be larger than the effect of the finite Trotter number, we did not attempt an extrapolation and gave the explicit value of the Trotter number in the figures.

For given $g$ and $\Omega$ the mean phonon occupation number is expected to grow with increasing temperature. As can be seen in Fig. 3.10 this is indeed the case. At low temperatures, however, a peak followed by a rapid decrement occurs. But this is only an effect of the finite Trotter number, it disappears with growing $M$.

In Fig. 3.11 we show the results for $\Omega=2 J, g=1.0$ and $N=16,32,64,128$. The 


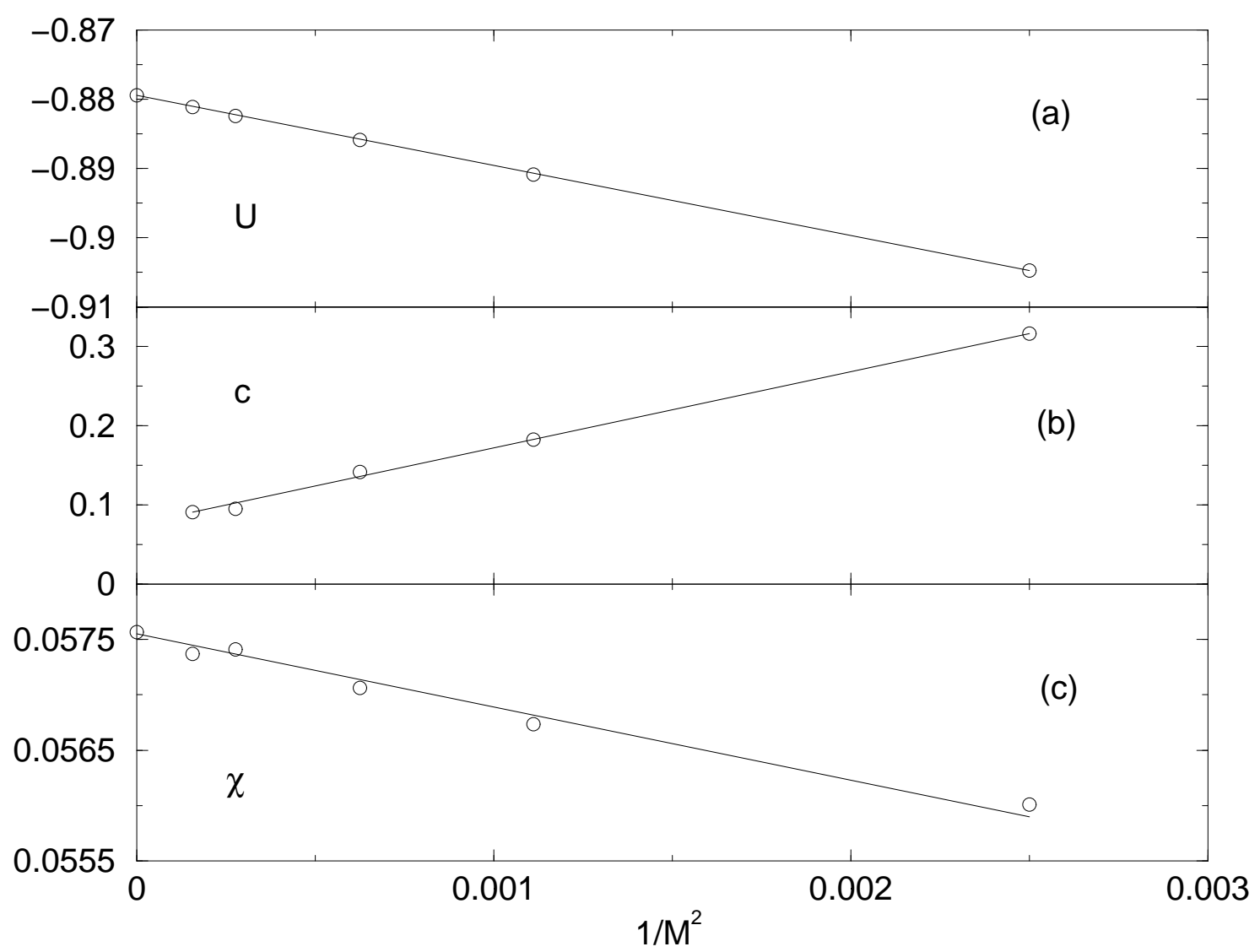

Figure 3.5: Internal energy $U /(J N)$, specific heat $c / N$, and magnetic susceptibility $\chi J / N$ of the Heisenberg model as a function of the inverse Trotter number squared. The temperature is $T=0.2 \mathrm{~J}$, the number of spin-updates is $10^{6}$, the chain length is $N=64$, and the examined Trotter numbers are $M=20,30,40,60,80, \infty$.

tendency of the magnetic susceptibility appears to approach a finite value at $T \rightarrow 0$. (Actually, a gap appears at very low temperatures, because as we will see later, the system with this choice of parameters is dimerized). The sharp decrease in the curves for $N=16,32,64$ is a finite-size effect, i. e., the energy gap causing the drop results from the discreteness of the spectrum of the finite system. It is not an intrinsic property of the spin chain.

In the following chapter we will examine the thermodynamic properties of spin-phonon chains. It will turn out that we can examine with very good accuracy especially those systems which have a phonon frequency which is at least as large as the spin-spin coupling constant. This is because these systems require a smaller configuration space than systems with a smaller phonon frequency. Systems with small phonon frequency have previously been supposed to generate the spin-Peierls phase transition, because the phonons of the organic spin-Peierls materials appear to be rather soft. However, this situation 

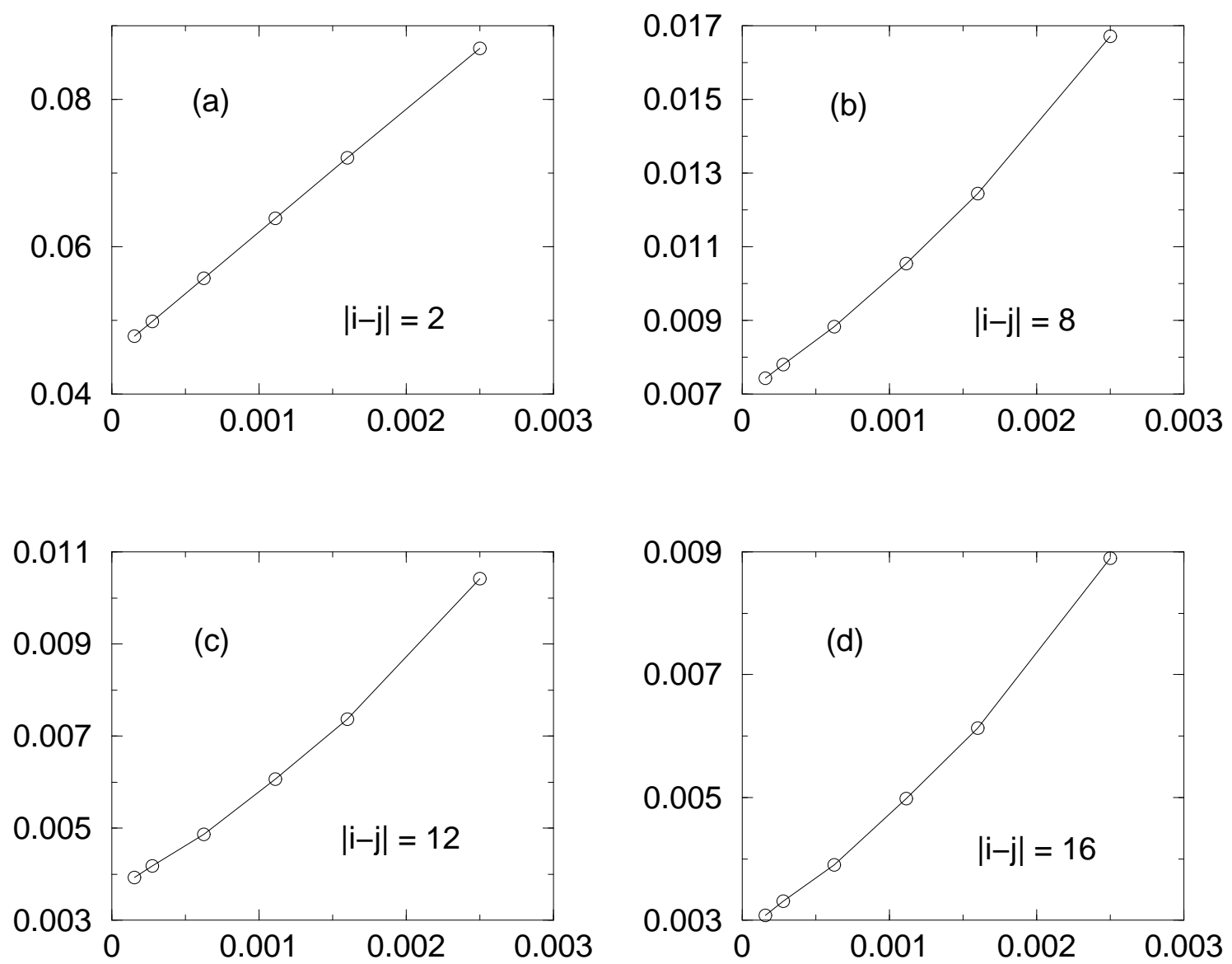

Figure 3.6: Spin-spin correlation $\left\langle S_{i}^{z} S_{j}^{z}\right\rangle$ of the spin-phonon model with phonon frequency $\Omega=2 J$ and spin-phonon coupling constant $g=1.0$ as a function of the inverse Trotter number squared. The temperature is $T=0.2 \mathrm{~J}$, the number of spin-updates is $10^{6}$, the chain length is $N=32$, and the examined Trotter numbers are $M=20,25,30,40,60,80$. We show the results for mutual distances $|i-j|$ of the sites of $2,8,12$, and 16 , respectively.

has dramatically changed with the discovery that the spin-Peierls transition generating phonons of the inorganic material $\mathrm{CuGeO}_{3}$ are at least as large as the spin-spin coupling constant $J$. Approximate values are $\Omega_{1} \sim 2 J$ and $\Omega_{2} \sim 4 J$.

Furthermore, we will see in the following chapter that large deviations of the spinphonon systems from the Heisenberg chain will occur only where large spin-phonon couplings are considered. This is the reason why in Figs. $3.6-3.11$ we are investigating the dependence on the finite Trotter of a model with the parameters $\Omega=2 J$ and $g=1.0$.

In Fig. 3.7 we examine dependence on the finite Trotter number of the spin-spin correlation function for a model with these parameters and at the temperature $T=0.2 J$. The statistical fluctuations are at least as large as the systematical effects of the finite Trotter number for a model with chain length $N=64$ and Trotter number $M=80$, where $10^{6}$ spin updates and $2 \times 10^{7}$ phonon updates have been used. This result is important, 

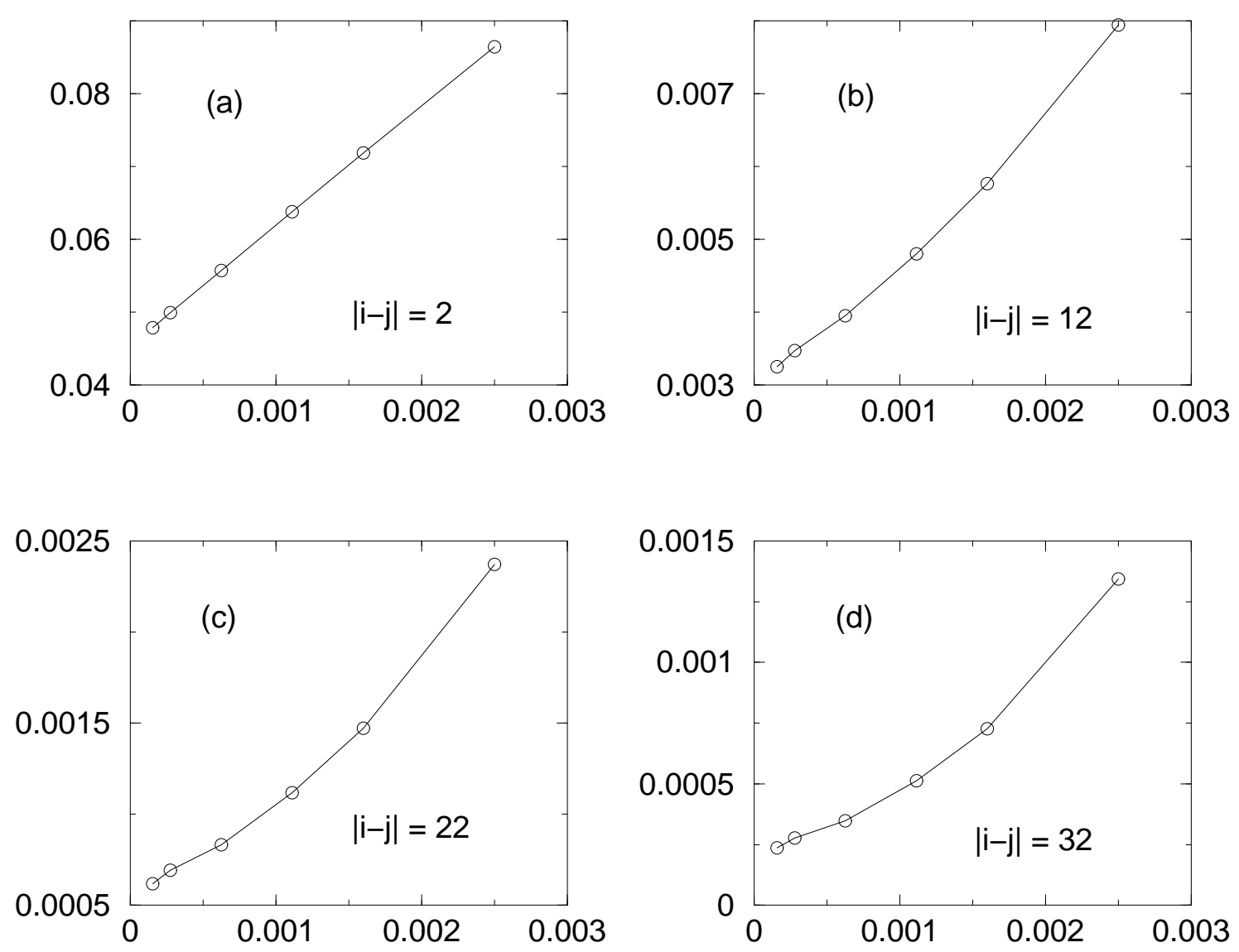

Figure 3.7: Like in Fig. 3.6 we show the spin-spin correlation $\left\langle S_{i}^{z} S_{j}^{z}\right\rangle$ as a function of the inverse Trotter number squared. Here we present the data for the chain length $N=64$ instead of $N=32$. Phonon frequency, spin-phonon coupling, temperature, the number of spin-updates, and the examined Trotter numbers are identical. For large mutual distances $|i-j|$ the spin-spin correlations for $N=32$ are much larger than those for $N=64$. Evidently, this is a pure finite size effect and means that $N=32$ chains cannot be used for the examination of systems with temperatures $T \leq 0.2 J$.

because when in the later discussion we will examine a possible power-law behavior of the correlation length as a function of the temperature we will use systems with $\Omega=2 J$, $g \leq 1.0$ in the temperature range $0.2 J \leq T \leq J$. Hence, effects of the finite Trotter number are clearly negligible for these systems in this temperature range.

The same is true for the magnetic susceptibility of this system $(\Omega=2 J$ and $g=1.0)$ in the temperature range $T \geq 0.2 J$ as can be inferred from Figs. 3.9c and 3.11. The reason is that the spin-gap of this system (with $N \geq 64$ ) is smaller than the temperatures investigated. Moreover the effects of the finite Trotter number are negligible for this system in the temperature region $T \geq 0.2 \mathrm{~J}$, provided that the Trotter number is chosen as large as $M \geq 80$.

To conclude, both the effects of finite size and finite Trotter number are negligible 

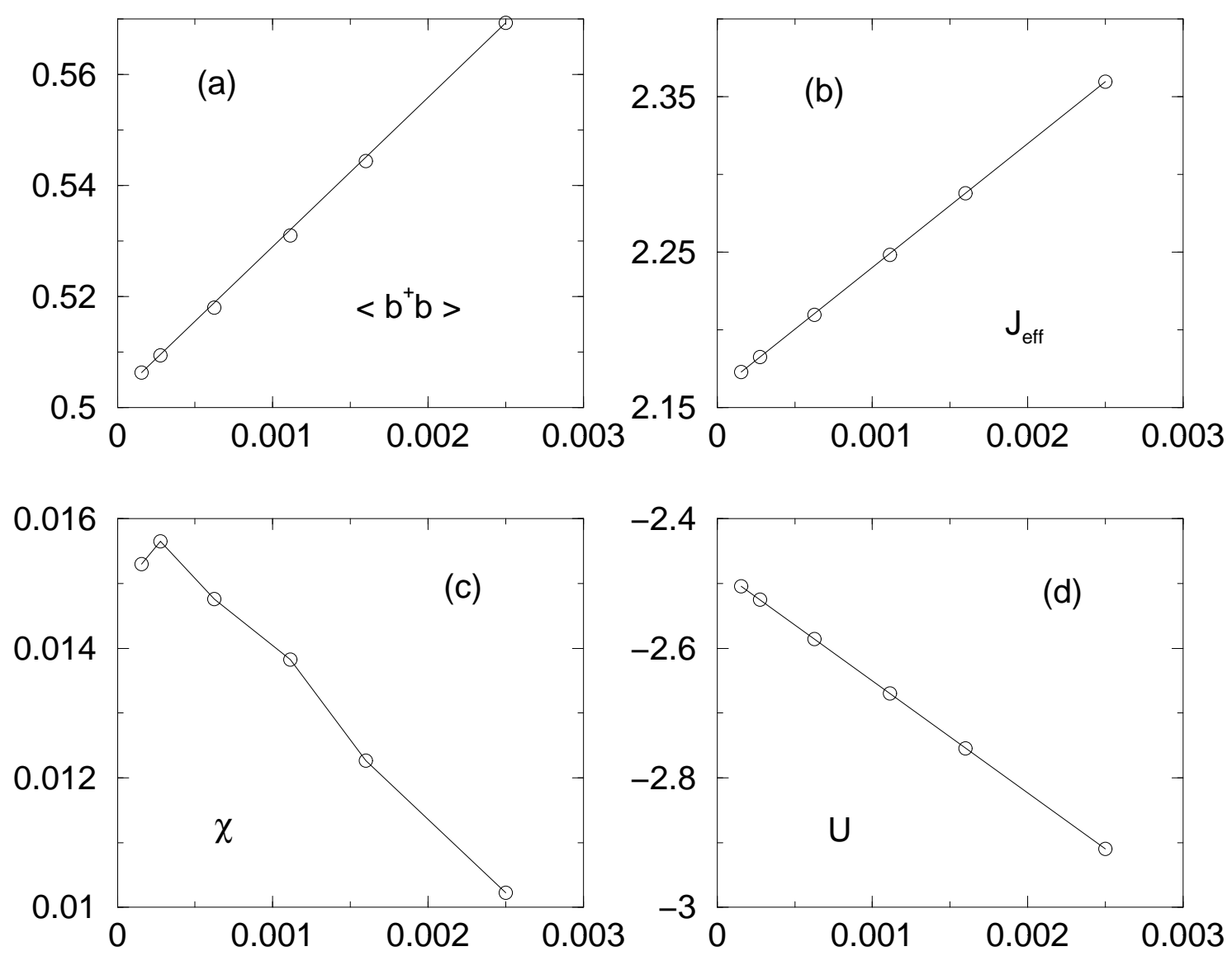

Figure 3.8: (a) Mean phonon occupation number per site, (b) effective spin-spin coupling $J_{\text {eff }}(T) / J$, (c) magnetic susceptibility $\chi J / N$, and (d) internal energy $U /(J N)$ of the spinphonon model with phonon frequency $\Omega=2 J$ and spin-phonon coupling constant $g=1.0$ as a function of the inverse Trotter number squared. The temperature is $T=0.2 \mathrm{~J}$, the number of spin-updates is $10^{6}$, the chain length is $N=32$, and the examined Trotter numbers are $M=20,25,30,40,60,80$.

when systems with $\Omega \geq 2 J$ and $g \leq 1.0$ are investigated for $N \geq 64$ and $M \geq 80$ in the temperature range $T \geq 0.2 J$. 

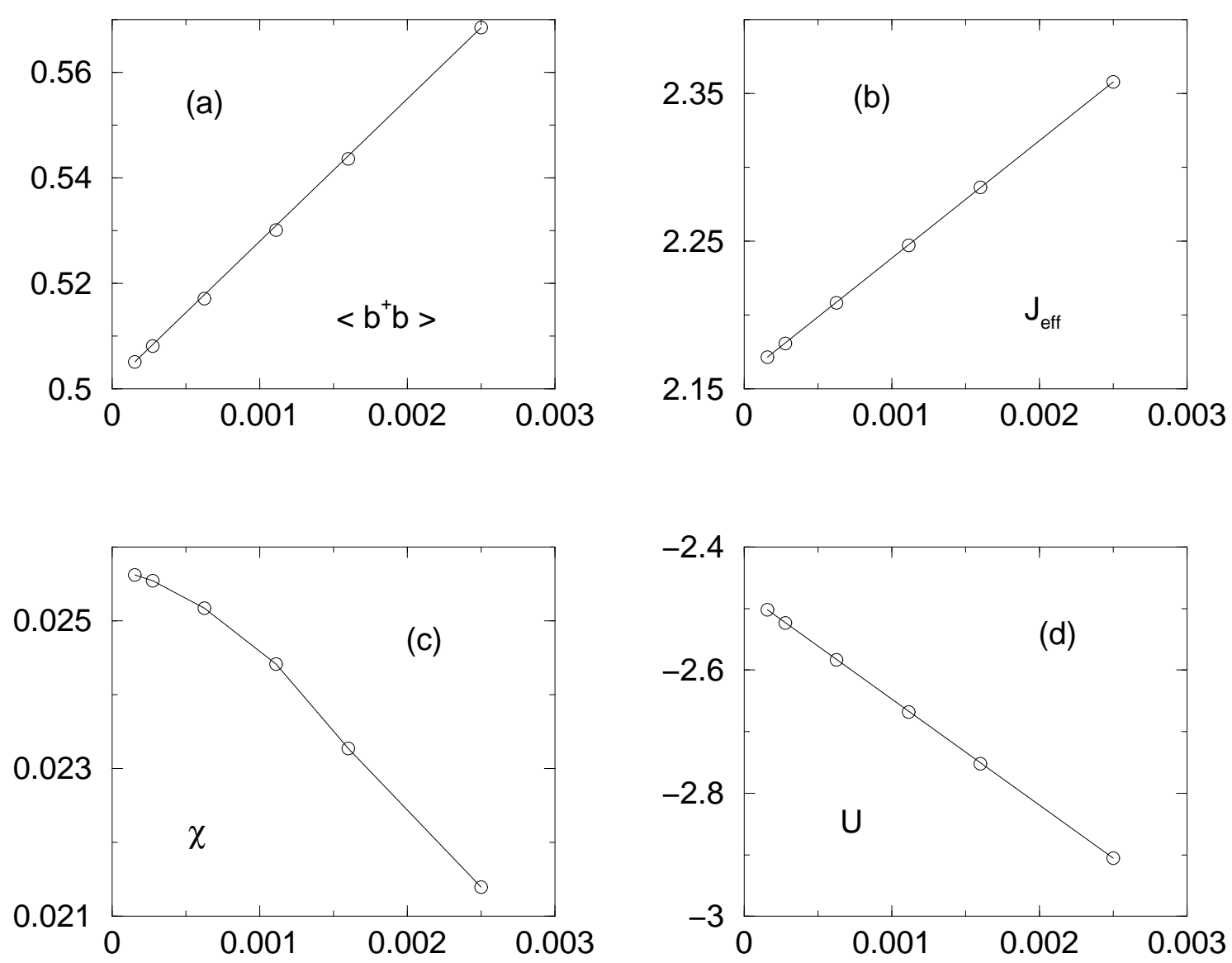

Figure 3.9: The same quantities as in Fig. 3.8. However, here we show data for the larger chain length $N=64$ instead of $N=32$. Remarkably, (a) the mean phonon occupation number per site, (b) the effective spin-spin coupling $J_{\text {eff }}(T) / J$, and (d) the internal energy $U /(J N)$ do not differ significantly for these two chain lengths. Finite size effects are significant only for (c) the magnetic susceptibility $\chi J / N$. The susceptibility is much larger for $N=64$ than for $N=32$. This results from the appearance of the finite size gap at low temperatures. 


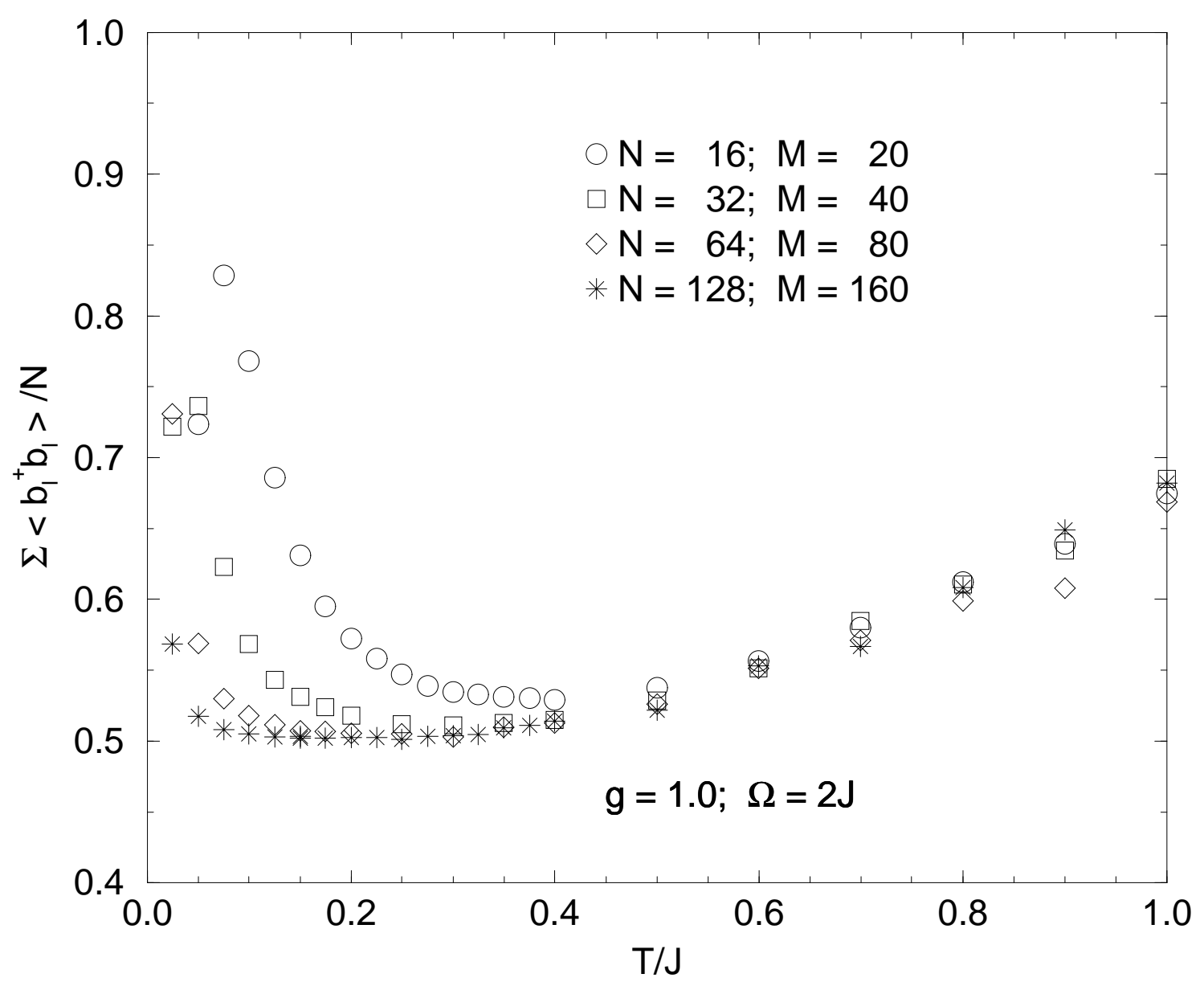

Figure 3.10: Mean phonon occupation number per site, $\frac{1}{N} \sum_{l=1}^{N}\left\langle b_{l}^{\dagger} b_{l}\right\rangle$, versus temperature. The spin-phonon coupling constant is $g=1.0$, and the phonon frequency is $\Omega=2 J$. Chain length $N$ and the Trotter number $M$ are varied over a wide range. The peak at low temperatures is an effect of the finite Trotter number, see Fig. 3.9. Note the drop for the lowest temperature in the cases of $N=16$ and $N=32$. 


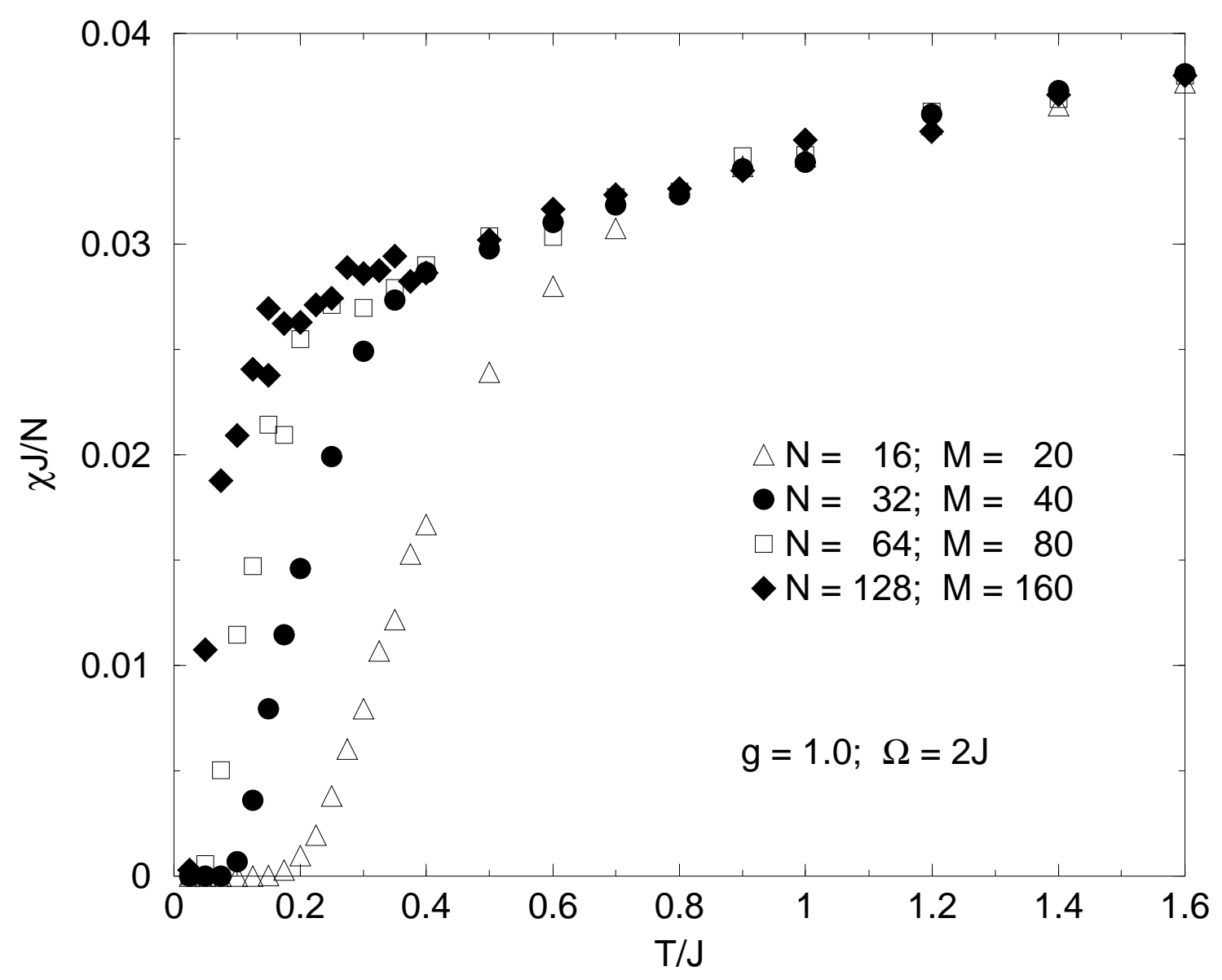

Figure 3.11: Magnetic susceptibility versus temperature. The spin-phonon coupling constant is $g=1.0$, and the phonon frequency is $\Omega=2 J$. Chain length $N$ and the Trotter number $M$ are varied over a wide range. The drop of the susceptibility at low temperature is a finite-size effect for $N=16,32,64$. Probably, this system has a spin gap. However, it is much smaller than the finite-size gap even for $N=64$. 


\section{Chapter 4}

\section{Thermodynamic properties}

\subsection{Magnetic susceptibility}

In the following we investigate how the spin-phonon coupling influences the magnetic susceptibility. Our observations can be summarized in the following three points:

- For fixed phonon frequency $\Omega$, the overall height of the magnetic susceptibility is lowered with increasing spin-phonon coupling. This is shown for $\Omega=2 J$ in Fig. 4.1 and for $\Omega=J$ in Fig. 4.2. - This observation is not surpising, because an increasing spin-phonon coupling is expected to decrease the magnetic order.

- For fixed spin-phonon coupling $g$, the susceptibility is growing with increasing phonon frequency $\Omega$ (see Fig. 4.3). - This is because for $\Omega \gg g J$ the magnetic degrees of freedom decouple. Hence, for $\Omega \rightarrow \infty$ (and $g J$ fixed) the magnetic properties are again dominated by the antiferromagnetic Heisenberg model.

- The maxima of the susceptibility curves are shifted towards higher temperatures when either the spin-phonon coupling increases or the phonon becomes softer. This observation is of great phenomenological importance, because it means that for substances where the spin-phonon coupling cannot be neglected, the value of the spin-spin coupling is overestimated, if determined by comparing the experimental data with the susceptibility calculated for the pure spin model. The quantitative values for this overestimation are shown in Fig. 4.7. We find the effective coupling constant, $J_{\text {eff }}^{g} \simeq J\left(1+1.8 g^{2} J / \Omega\right)$.

The local phonon displacement is defined as

$$
\delta_{l}=g\left\langle b_{l}^{\dagger}+b_{l}\right\rangle
$$

In the adiabatic limit it corresponds to the dimerization in a Heisenberg model with alternating couplings. Its average over all sites,

$$
\delta=\frac{1}{N} \sum_{l=1}^{N} \delta_{l}
$$




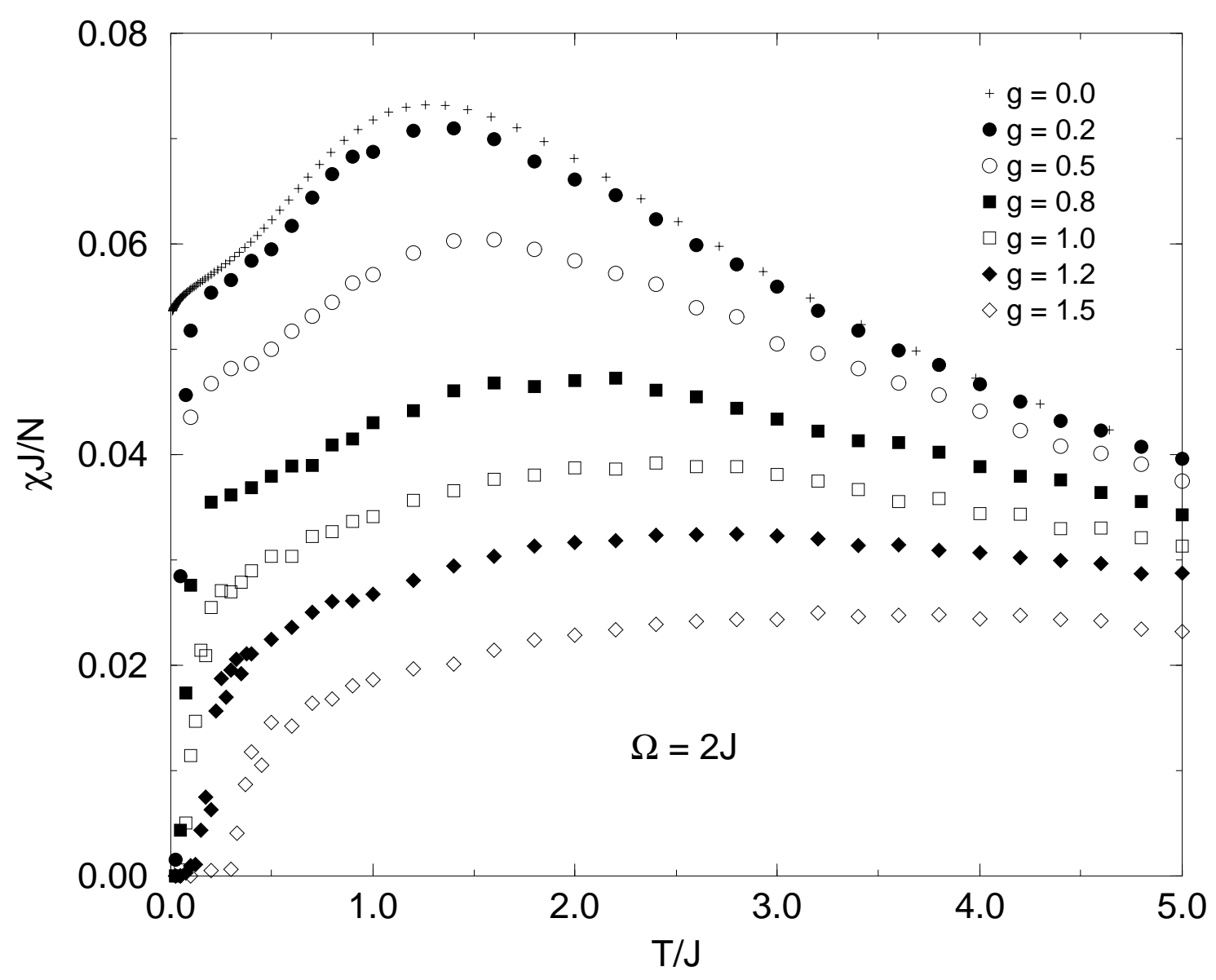

Figure 4.1: Magnetic susceptibility versus temperature for $\Omega=2 J$ and spin-phonon coupling $g$ between 0.2 and 1.5. For low temperatures, $T \leq J$, the chain length is $N=64$ and the Trotter number is $M=80$. For high temperatures we used $N=16$ and $M=20$. For comparison we show the exact results for the Heisenberg chain (data from A. Klümper).

represents the uniform phonon displacement and is closely related to the effective spinspin-coupling [7, 42],

$$
J_{\mathrm{eff}}(T)=J(1+\delta) .
$$

For all our choices of $g$ and $\Omega, J_{\text {eff }}$ is a slowly decreasing function of the temperature, see Fig. 4.4. This behavior has been noted before for $g=1 /(4 \sqrt{2})$ and $\Omega=2 J$ in Ref. [7]. This finding is also in agreement with Ref. [106], where a comparison of inelastic neutron scattering data on $\mathrm{CuGeO}_{3}$ with calculated dynamic structure factors $S(q, \Omega)$ gave evidence for a decrease of the spin-coupling with increasing temperature. A similar behavior of the spin-coupling has been reported in Ref. [107]. 


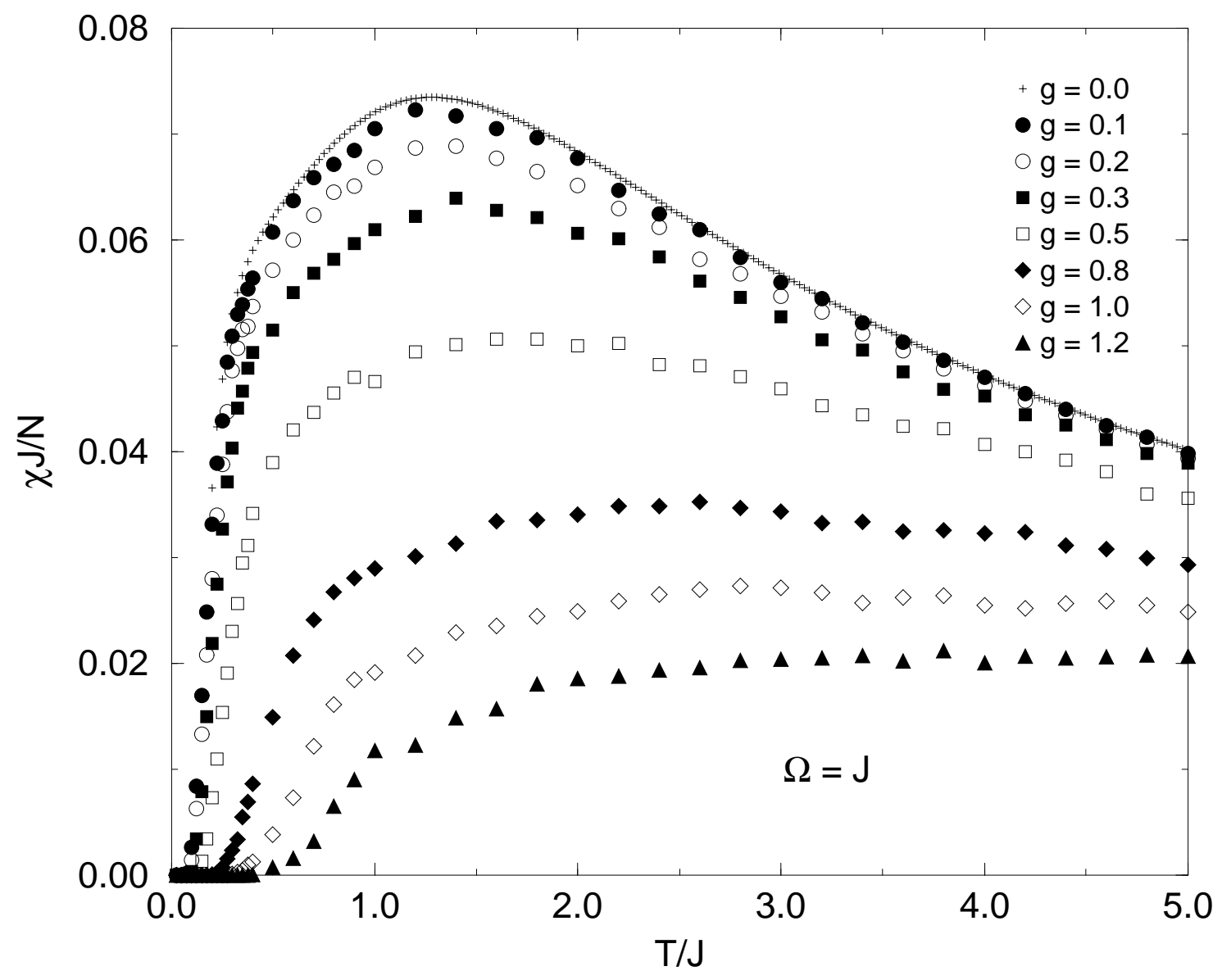

Figure 4.2: Similar to Fig. 4.1 we show the magnetic susceptibility versus temperature. Here, however, the phonon frequency is smaller, $\Omega=J$. The spin-phonon coupling $g$ is between 0.1 and 1.2. The chain length is $N=16$ and the Trotter number is $M=20$. For comparison we show the exact results for the Heisenberg chain with $N=16$ (data from U. Löw). For given $g$ and $T$, the magnetic susceptibility is smaller for $\Omega=J$ than for $\Omega=2 J$.

For fixed temperature, the displacement $\delta$ is a function of the phonon frequency and the spin-phonon coupling, we found (see Fig. 4.5)

$$
\delta=f(T) \frac{g^{2} J}{\Omega} .
$$

The proportionality constant is itself a function of the temperature, consistent with the temperature dependence of $J_{\text {eff. }}$. We found

$$
\begin{aligned}
f(T=0.3 J) & =2.38 \pm 0.04 \\
f(T=J) & =2.28 \pm 0.02 .
\end{aligned}
$$




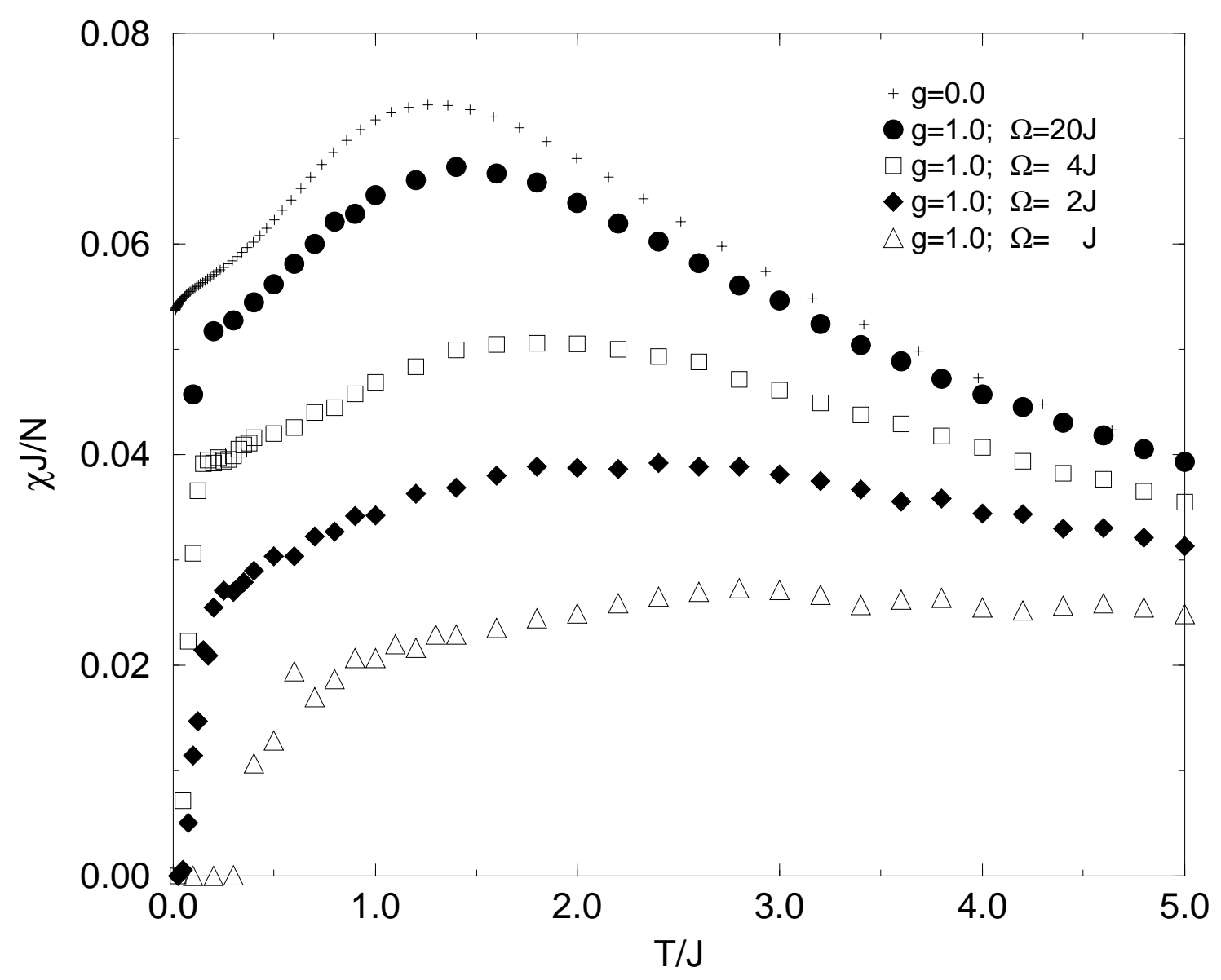

Figure 4.3: Magnetic susceptibility versus temperature for $g=1.0$ and phonon frequency between $\Omega=J$ and $\Omega=20 J$. The number of sites is $N=64$ (and $M=80$ ) for low temperatures, $T \leq J$ ( $T \leq 1.5 J$ for the $\Omega=J$ chain), whereas $N=16$ and $M=20$ for high temperatures. For $g=0$ we show the exact data of A. Klümper.

This result is in qualitative agreement with observations by Wellein et al. [108], who found a lowering of the dimerization with growing phonon frequency at $T=0$. It also confirms calculations by Pytte [42] and Uhrig [102], who found the spin-phonon Hamiltonian (for translationally invariant phonons) to be equivalent to an effective spin Hamiltonian with effective spin-spin coupling $J_{\text {eff }}=J\left(1+g^{2} J / \Omega\right)$.

The rescaled susceptibilities, $\chi(J, g, \Omega, T) J_{\text {eff }}(T)$ are shown in Fig. 4.6 as a function of $T / J_{\text {eff }}(T)$. The rescaled susceptibilities do not fall together with the susceptibility of the Heisenberg chain. Hence, the effective spin-spin coupling $J_{\text {eff }}(T)$ cannot be used to bring the curves with different parameters together. The effective spin-spin coupling as defined above is not the correct variable to rescale the thermodynamic observables.

However, to high precision we find that the susceptibilities can be rescaled by a global and temperature independent $J_{\text {eff, }}^{g}$, see Fig. 4.7. Naturally, this rescaling cannot be applied 


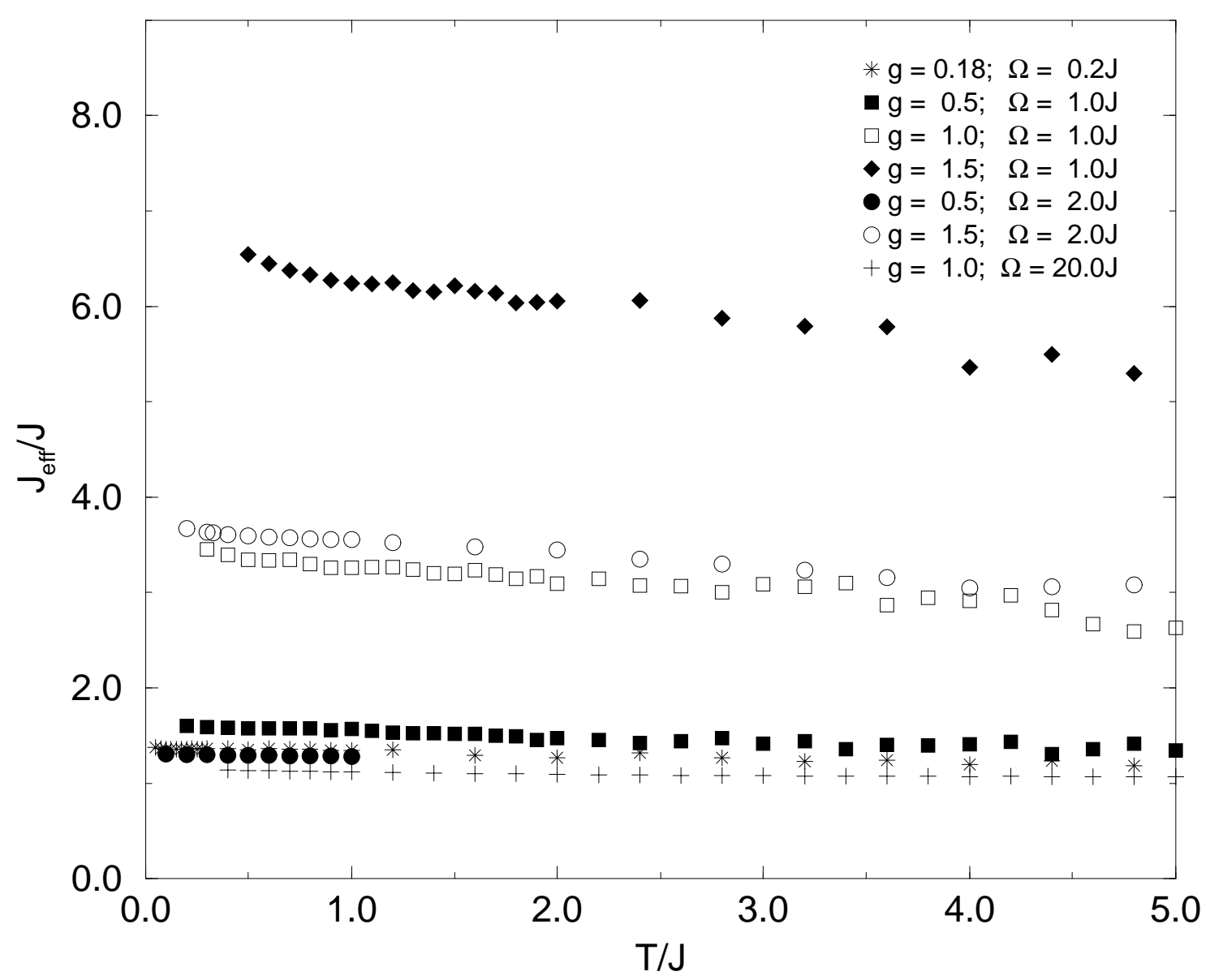

Figure 4.4: Effective spin-spin coupling $J_{\mathrm{eff}}(T) / J$ versus temperature for various sets of $(g, \Omega)$. Here, $J_{\text {eff }}(T)=J\left(1+(g / N) \sum_{l=1}^{N}\left\langle b_{l}^{\dagger}+b_{l}\right\rangle\right)$. The decrement of $J_{\text {eff }}(T) / J$ with temperature confirms an earlier observation by Sandvik et al. [7].

to the low-temperature region of those spin-phonon systems which have a spin gap.

We found

$$
\chi(J, g, \Omega, T) J_{\mathrm{eff}}^{g}=J \chi^{\mathrm{AFH}}\left(T / J_{\mathrm{eff}}^{g}, J_{\mathrm{eff}}^{g}\right),
$$

where $J_{\text {eff }}^{g}$ is the global effective spin-spin coupling, by comparing the temperatures $T_{\max }(g, \Omega)$, where the susceptibilites of the spin-phonon chains have their maxima, at temperature $T_{\mathrm{max}}^{\mathrm{AFH}}$, where the susceptibility of the Heisenberg chain has its maximum. Hence,

$$
J_{\mathrm{eff}}^{g}=J \frac{T_{\max }(g, \Omega)}{T_{\max }^{\mathrm{AFH}}} .
$$

We would like to point out that, in contrast to Ref. [7], we do not require an overall Landé factor to obtain the scaling law. 

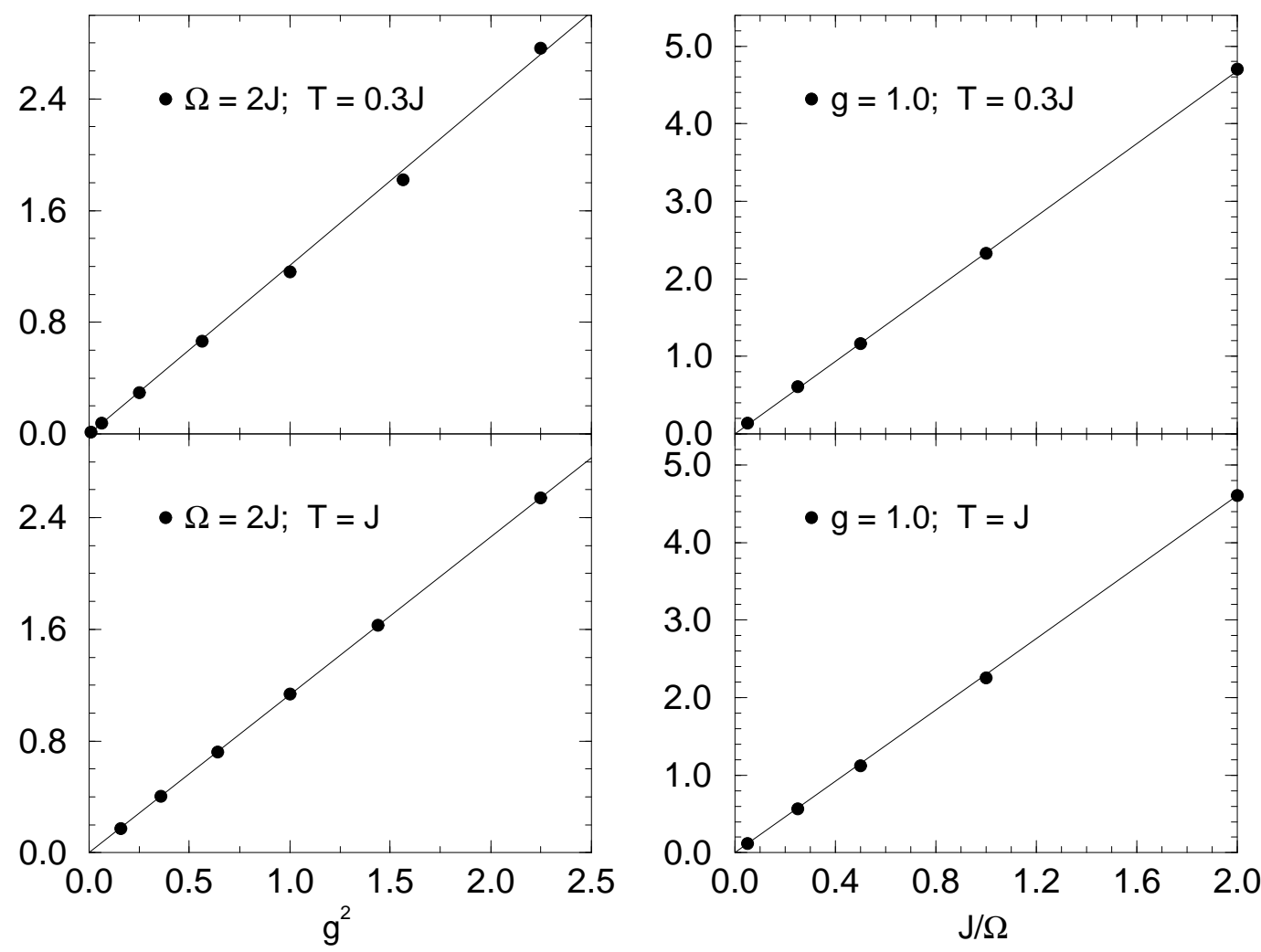

Figure 4.5: Displacement $\delta=J_{\text {eff }}(T) / J-1$ versus $g^{2}$ (left side) and versus $J / \Omega$ (right side) for $N=64$ and $M=80$.

From Fig. 4.7 we see that the coupling constant which is experimentally determined by fitting the susceptibility of the Heisenberg chain on a susceptibility curve of a real material is not the bare coupling constant $J$ but the effective coupling constant $J_{\text {eff }} \geq J$. The phenomenological equation

$$
J_{\mathrm{eff}}^{g} \simeq J\left(1+1.8 g^{2} J / \Omega\right)
$$

obtained from the data of Fig. 4.7 can be rewritten as,

$$
\frac{J_{\mathrm{eff}}^{g}}{J}=\frac{3.6 g^{2} J_{\text {eff }}^{g} / \Omega}{-1+\sqrt{1+7.2 g^{2} J_{e f f}^{g} / \Omega}} .
$$

As the experimentally determined ratios are not $\Omega / J$, as assumed previously [52, 93], but $\Omega / J_{\text {eff }}^{g}$, this equation will be helpful for the determination of the correct spin-spin coupling constant $J$. 


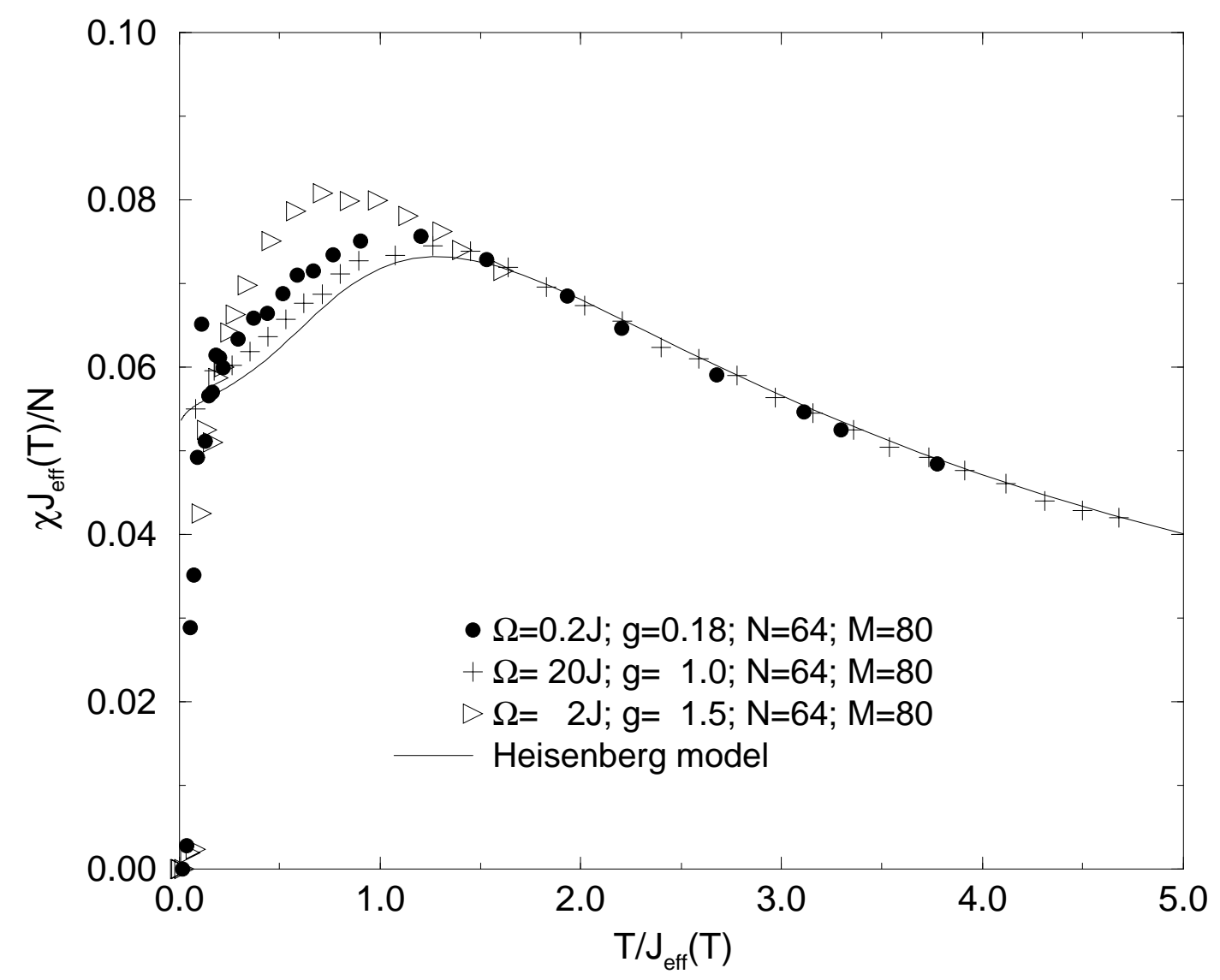

Figure 4.6: Rescaled susceptibilities $\chi(J, g, \Omega, T) J_{\text {eff }}(T) / N$ versus $T / J_{\text {eff }}(T)$. The rescaled susceptibilities differ strongly from one another, so that from a given curve one can easily conclude on the effective coupling $J_{\text {eff }}(T)$. Unfortunately, $J_{\text {eff }}(T)$ cannot be measured experimentally, so that at present this method is not yet applicable to experimental investigations which try to determine $g$ and $\Omega$.

A candidate for the generalization of Eq. (4.9) for a system with $n$ phonon frequencies is

$$
J_{\mathrm{eff}}^{g} \simeq J\left(1+1.8 \sum_{i=1}^{n} g_{i}^{2} J / \Omega_{i}\right)
$$

This equation can be rewritten as

$$
\frac{J_{\mathrm{eff}}^{g}}{J}=\frac{3.6 \sum_{i=1}^{n}\left(g_{i}^{2} J_{\text {eff }}^{g} / \Omega_{i}\right)}{-1+\sqrt{1+7.2 \sum_{i=1}^{n}\left(g_{i}^{2} J_{e f f}^{g} / \Omega_{i}\right)}} .
$$




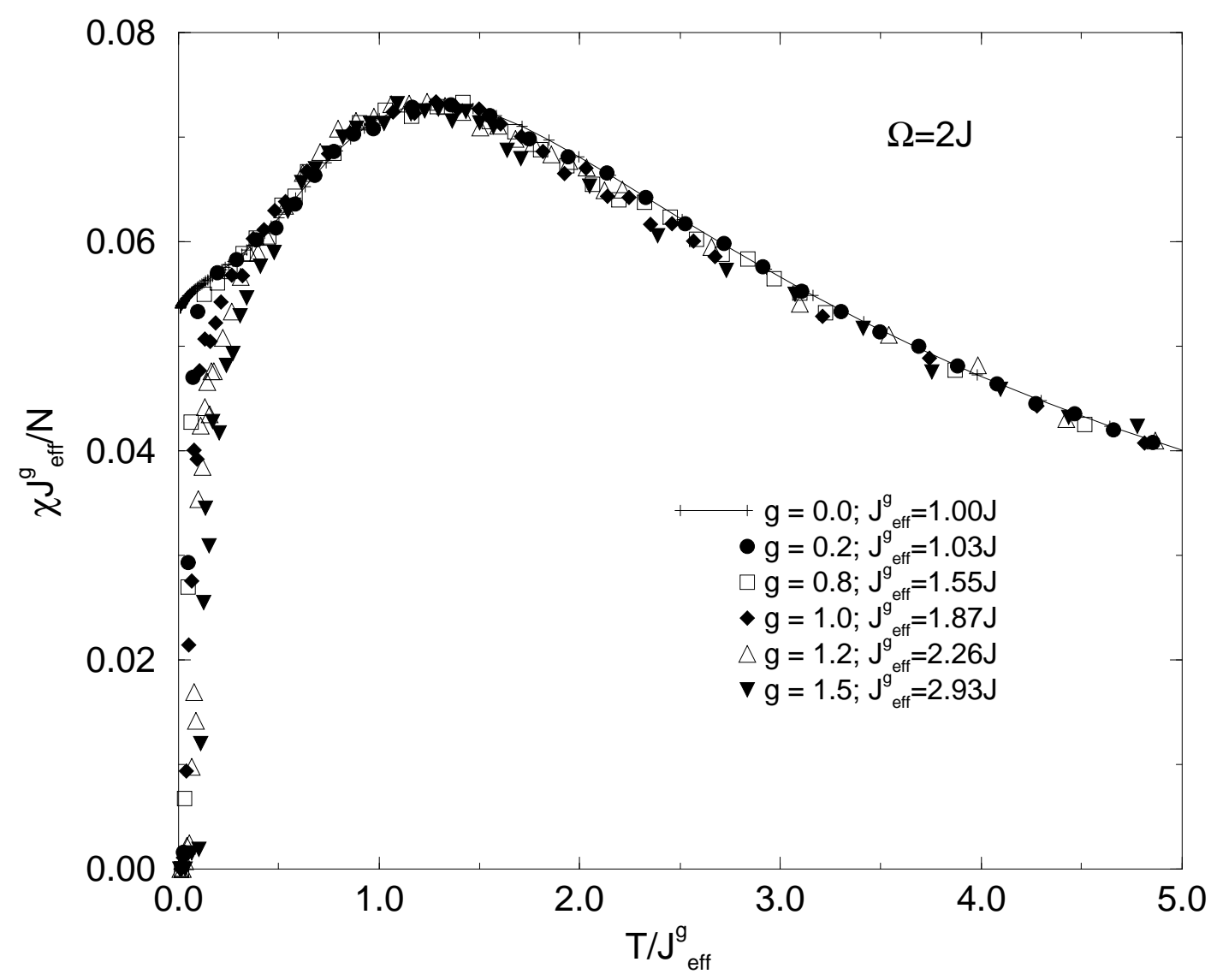

Figure 4.7: Rescaled susceptibilities $\chi(J, g, \Omega, T) J_{\text {eff }}^{g} / N$ versus $T / J_{\text {eff }}^{g}$. In contrast to Fig. 4.6 the magnetic susceptibilities are rescaled not by a temperature dependent effective coupling constant but by a global coupling constant. With very good agreement the hightemperature shape of the susceptibilities of spin-phonon Heisenberg chains can be mapped onto the rescaled susceptibility of the Heisenberg spin chain. Hence, the coupling constant which is experimentally determined by fitting the susceptibility of the Heisenberg chain on a susceptibility curve of a physical material is not the bare coupling constant $J$ but the effective coupling constant $J_{\text {eff }} \geq J$.

For $\mathrm{CuGeO}_{3}$ the phonon frequencies have been determined experimentally to be $\Omega_{1}=$ $2 J_{\text {eff }}^{g}$ and $\Omega_{2}=4 J_{\text {eff }}^{g}$, the spin-phonon coupling constants are roughly $g_{1}=-3 / 8$ and $g_{2}=3 / 2[52,93]$. As a consequence, we get $J_{\text {eff }}^{g} \simeq 1.68 J$ and therefore $\Omega_{1} \simeq 3.36 J$ and $\Omega_{2} \simeq 6.71 J$. 


\subsection{Mean phonon occupation number}

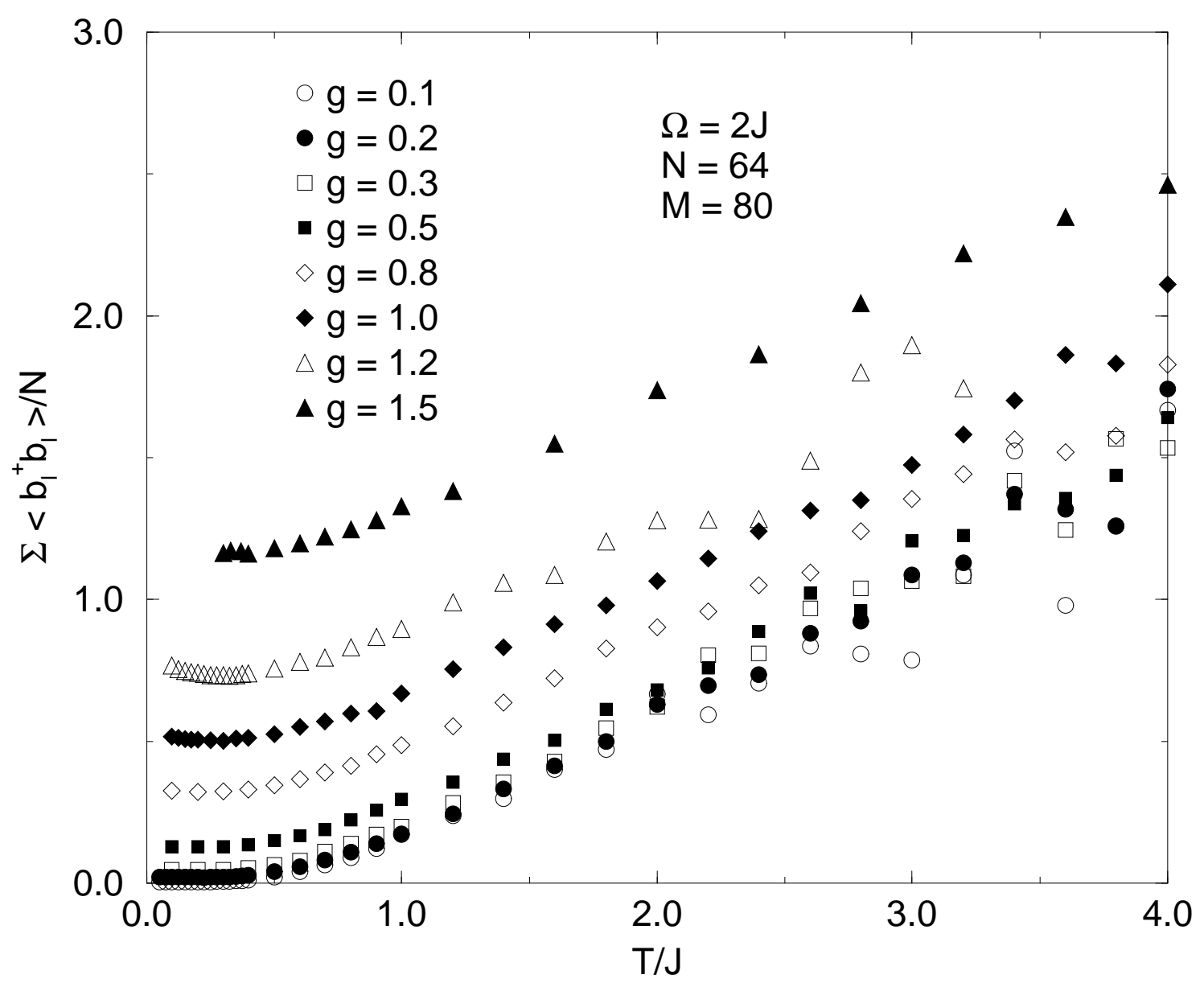

Figure 4.8: Mean phonon occupation number per site as a function of the temperature for $\Omega=2 J$. The spin-phonon coupling constant $g$ is between 0.1 and 1.5. For low temperatures the data show only small statistical fluctuations due to the small configuration space. Unfortunately, the configuration space increases rapidly with temperature which results in large statistical fluctuations for $T \geq 2 \mathrm{~J}$.

Let us investigate the behavior of the mean phonon occupation number per site,

$$
n(T)=\frac{1}{N} \sum_{l=1}^{N}\left\langle b_{l}^{\dagger} b_{l}\right\rangle .
$$

From Fig. 4.8 we see that for high temperatures, the mean phonon occupation number increases with temperature. However, at low temperatures it appears to approximate a constant nonzero value. When studying spin-phonon chains with various choices of 


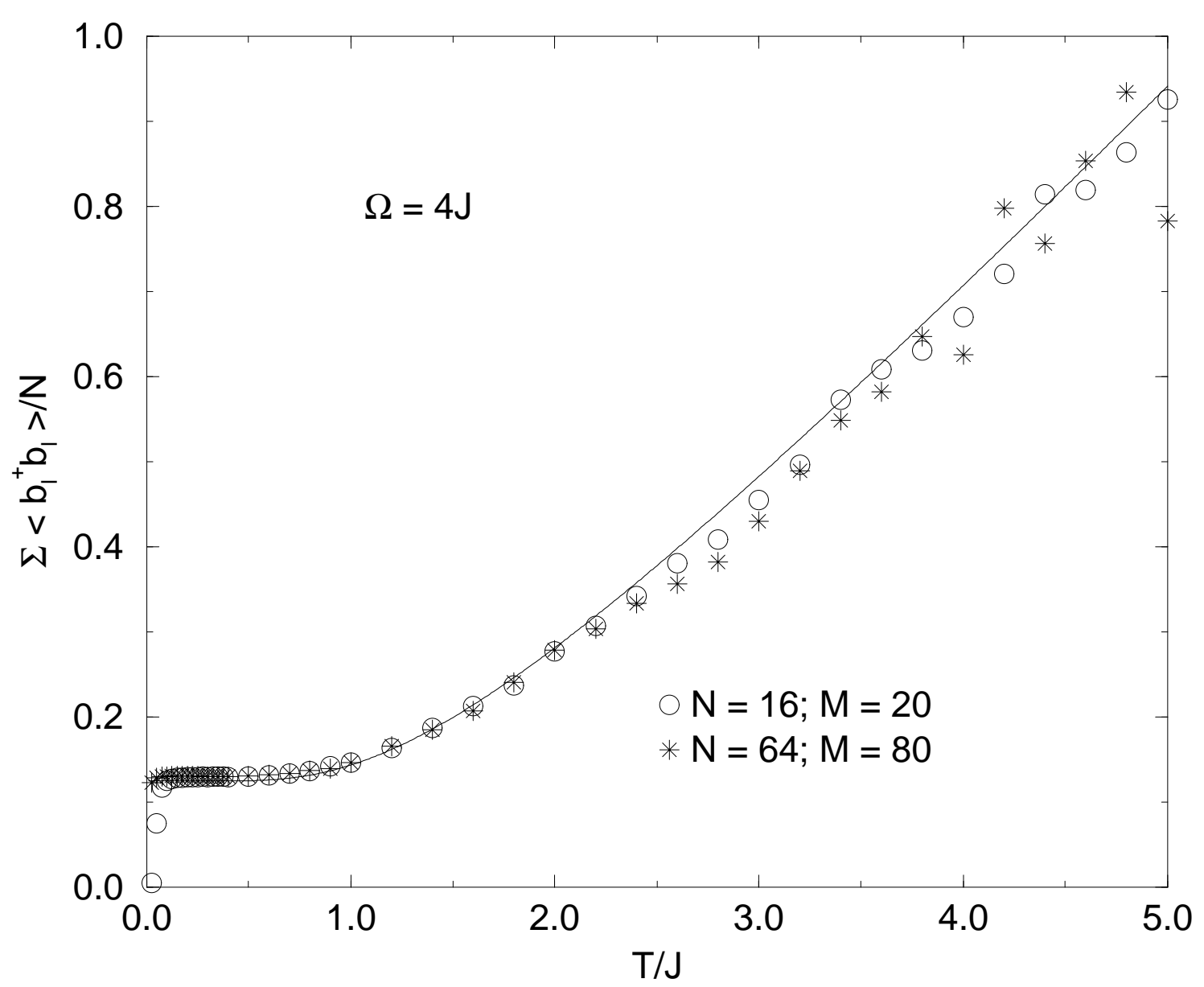

Figure 4.9: Mean phonon occupation number as a function of temperature for phonon frequency $\Omega=4 J$ and spin-phonon coupling constant $g=1.0$. The number of sites is 16 and 64 , respectively. The solid line shows $n_{f}(T)+n_{0}(T)$, where $n_{0}(T) \equiv n(T)-$ $n_{f}(T)$ was approximated by the temperature-independent value $n_{0}(0)=2(g J / \Omega)^{2}$. The statistical fluctuations are much smaller than the corresponding ones for the $\Omega=2 J$ systems, because the larger frequency reduces the mean phonon occupation number and the configuration space.

parameters $g$ and $\Omega$ at low temperatures, we find

$$
n_{0}=2\left(\frac{g J}{\Omega}\right)^{2},
$$

see Figs. 4.10-4.12. This is in accordance with observations by Augier et al. who found $n_{0}>0$ at $T=0$ for spin-phonon systems with the same Hamiltonian [95] and $n_{0} \propto g^{2} / \Omega^{2}$ for the dimerized spin chain [86]. For these systems Augier et al. examined only very small systems, the chain length was $N=4$ for the spin-phonon chain and $N=12$ for the dimerized model. 


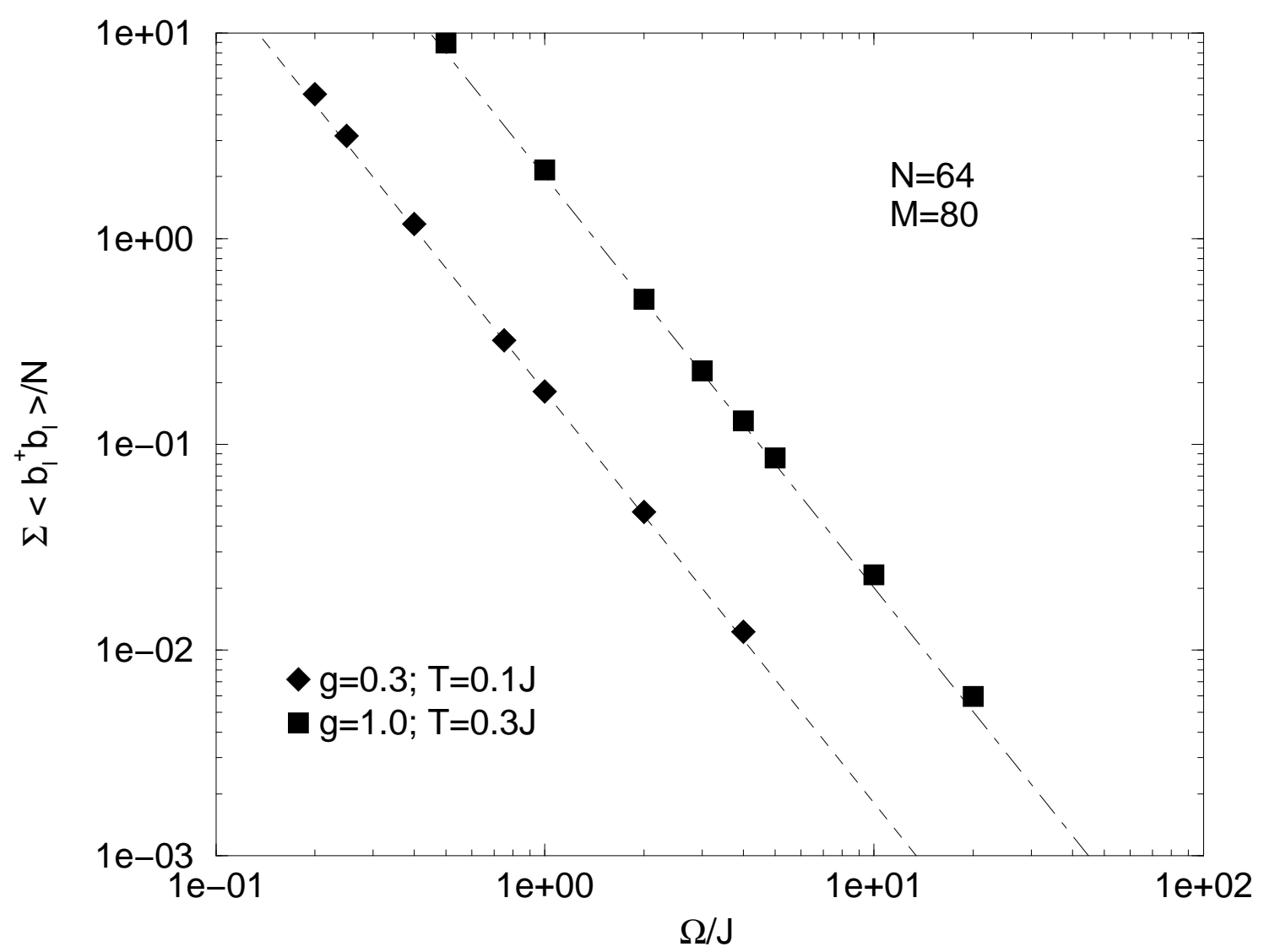

Figure 4.10: Mean phonon occupation number as a function of the phonon frequency $\Omega$ at fixed low temperature. The dashed lines show the relation $2(g J / \Omega)^{2}$. The small but finite temperatures were taken, because at even lower temperatures finite Trotter number effects significantly influence the behavior of the mean phonon occupation number.

The behavior at high temperatures can be understood by considering the contribution of the free Einstein phonons whose mean occupation number per site is (in a pure phononic system),

$$
n_{f}(T)=\frac{1}{\mathrm{e}^{\Omega / T}-1}
$$

Figure 4.9 shows the mean phonon occupation number $n(T)$ for a spin-phonon chain with $\Omega=4 J$ and $g=1.0$. For comparison, the solid line shows the contribution $n_{f}(T)$ of the free phonons plus the offset $n_{0}$. The occupation number $n(T)$ differs only slightly from $n_{f}(T)+n_{0}$. Hence, except from this small deviation, the spin-phonon coupling generates mainly the offset $n_{0}$ in the phonon occupation number.

The drop at low temperatures is clearly seen for $N=16$. Since it disappears for $N=64$, it turns out to be a finite-size effect. Note also that the mean phonon occupation number can be examined to very low temperatures for given chain length. 


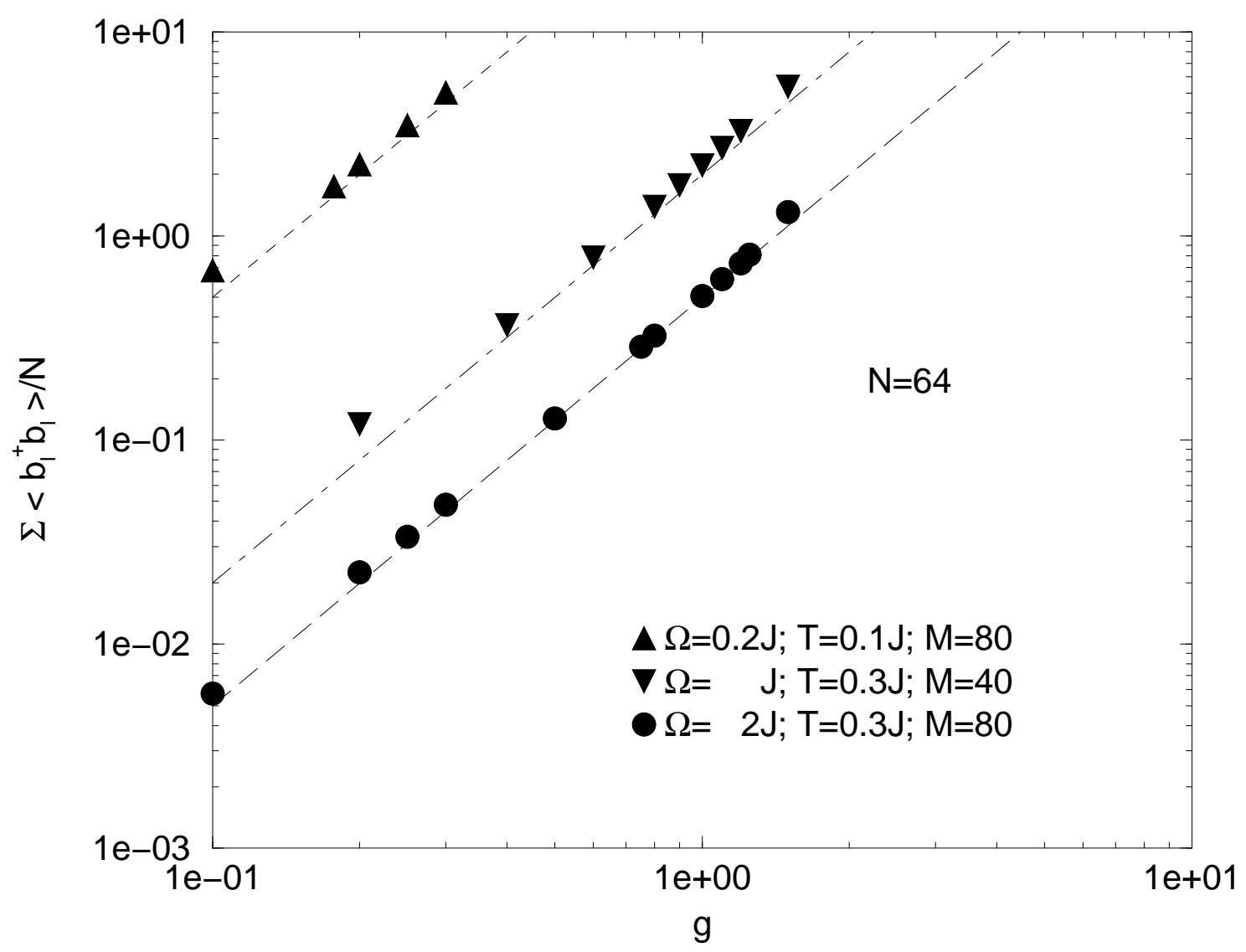

Figure 4.11: Mean phonon occupation number as a function of the spin-phonon coupling $g$ at fixed low temperature. The dashed lines show the relation $2(g J / \Omega)^{2}$. The small but finite temperatures were taken, because at even lower temperatures finite Trotter number effects significantly influence the behavior of the mean phonon occupation number.

The behavior at low temperatures can be understood as follows. The ground state determines the properties of the system at zero temperature, $T=0$, and dominates its properties at sufficiently low temperatures. The phononic part of the ground state of the system described by the Hamiltonian,

$$
\mathcal{H}=\frac{J}{2} \sum_{l=1}^{N}\left(\vec{\sigma}_{l} \vec{\sigma}_{l+1}-1\right)\left(1+g\left(b_{l}^{\dagger}+b_{l}\right)\right)+\Omega \sum_{l=1}^{N} b_{l}^{\dagger} b_{l},
$$

can be found by considering the local phononic part only,

$$
\mathcal{H}_{p, l}=\frac{J g}{2}\left(b_{l}^{\dagger}+b_{l}\right)+\Omega b_{l}^{\dagger} b_{l} .
$$

By defining

$$
\tilde{b}_{l}^{(\dagger)}:=b_{l}^{(\dagger)}+\frac{J g}{2 \Omega}
$$




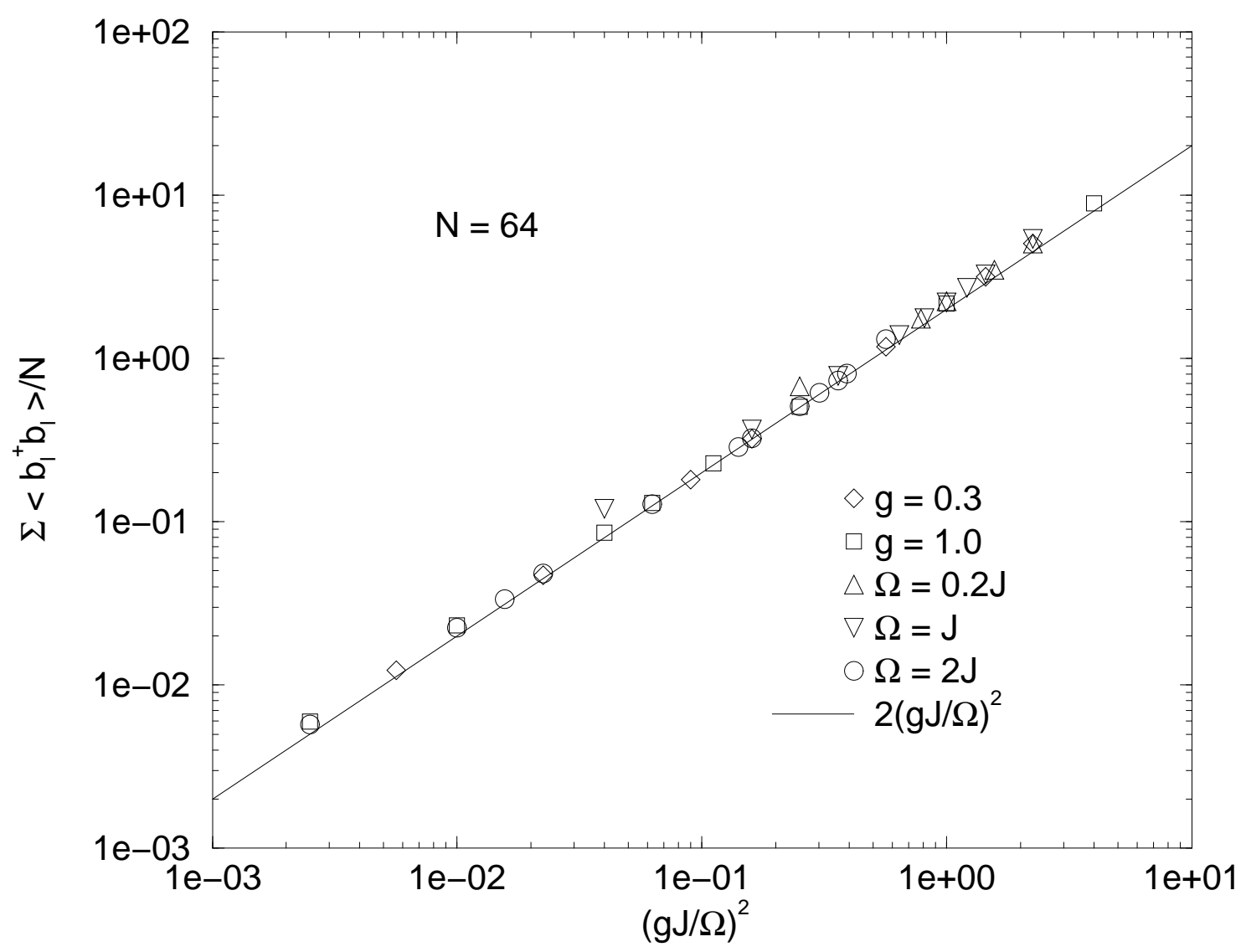

Figure 4.12: Mean phonon occupation number versus $(g J / \Omega)^{2}$ at fixed low temperature. The dashed line shows the relation $2(g J / \Omega)^{2}$. The data points lie systematically above the dashed line, because of the finite temperature effects. Apart from these small effects, the claimed relation is obviously satisfied.

we can rewrite the local phononic part as,

$$
\mathcal{H}_{p, l}=\Omega \tilde{b}_{l}^{\dagger} \tilde{b}_{l}-\frac{1}{\Omega}\left(\frac{J g}{2}\right)^{2} .
$$

The new operators $\tilde{b}_{l}^{(\dagger)}$ are Bose operators and describe phonons, because they satisfy

$$
\left[\tilde{b}_{l}^{\dagger}, \tilde{b}_{j}^{\dagger}\right]=\left[b_{l}^{\dagger}, b_{j}^{\dagger}\right]
$$

By using the normalization $\langle 0 \mid 0\rangle=1$ we find

$$
\left\langle 0\left|\mathcal{H}_{p, l}\right| 0\right\rangle=-\frac{1}{\Omega}\left(\frac{J g}{2}\right)^{2}
$$

This equation shows the correct proportionalities for the local phonon displacement and the low-temperature behavior of the mean phonon occupation number. 


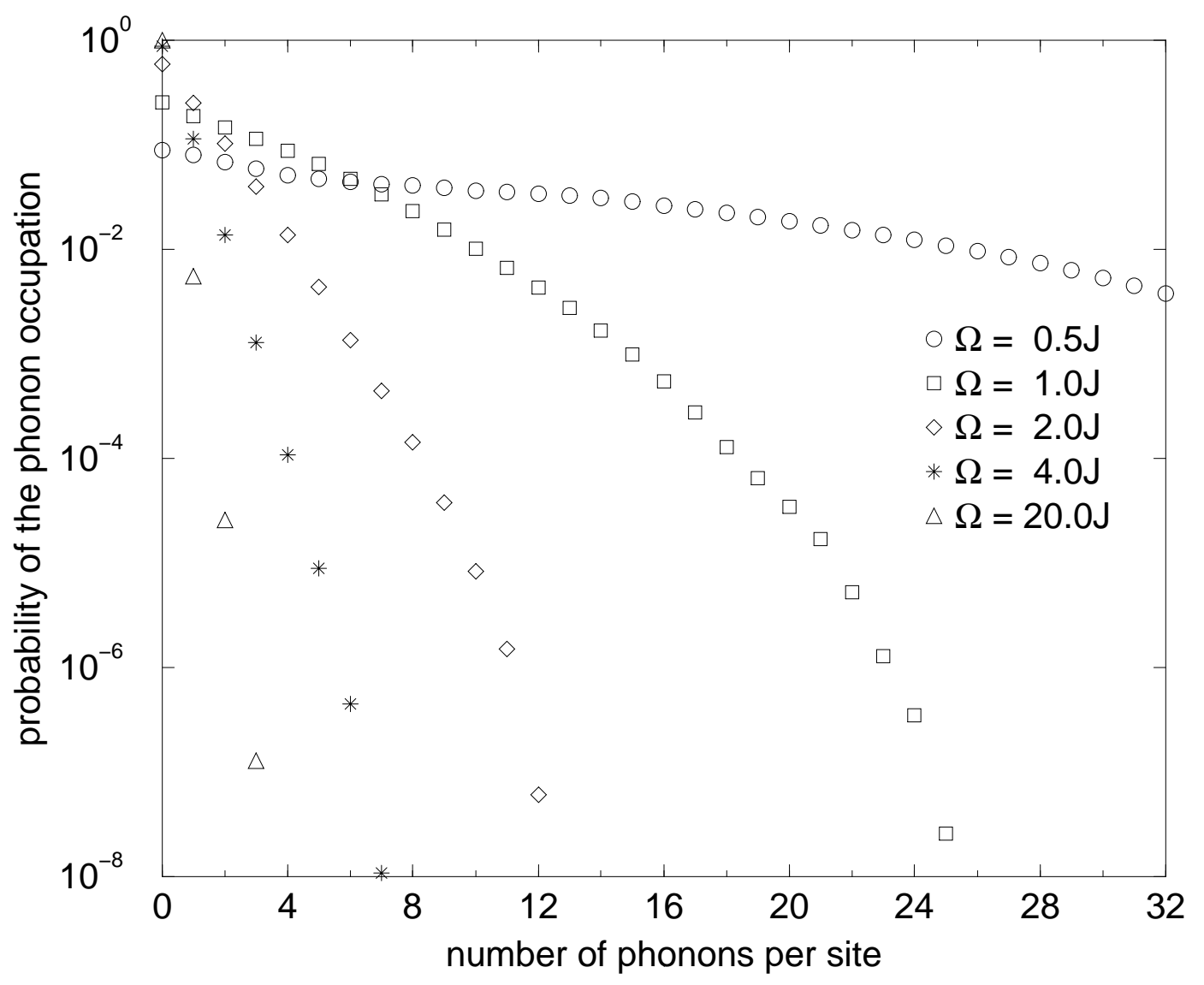

Figure 4.13: Probability of the phonon occupation number for phonon frequencies between $\Omega / J=0.5$ and 20 for a chain with $N=64$ sites, Trotter number 80, spin-phonon coupling constant $g=1.0$, and temperature $T=J$.

Truncation errors are a systematical source of error. For example, they cannot be overcome by the Lanczos method. As the dimension of the subspaces is quickly growing with the number of phonons the limits of a method requiring truncation is soon reached. This is relevant when using exact diagonalization which allows the treatment of up to only $\sim 16$ spins and a few phonons per site. At zero temperature the truncation problem can be partly overcome by using coherent phonon states $[88,100]$ or employing the DMRG technique, which allows the treatment of systems with sizes up to 256 spins [8].

At finite temperatures, however, the truncation problem is even more important. With the probability distribution of the phonon occupation number shown in Fig. 4.13 it is dramatically demonstrated that high cutoffs are essential when studying finite temperature properties. For a chain with $\Omega \geq J$ and $g=1.0$ at $T \leq J$ a truncation at $\sim 30$ phonons 
per site is required. (The probability for more phonons per site is less than $10^{-7}$.) For $\Omega / J \leq 0.5$ the truncation has to be $\geq 50$ phonons per site. Only very high phonon frequencies, i. e. $\Omega \geq 20 J$ can be treated properly with only 4 phonons per site. With our method, however, we can overcome the truncation errors for most of our systems. The truncation we choose for most of our systems is $\geq 50$ phonons per site.

\subsection{Specific heat}

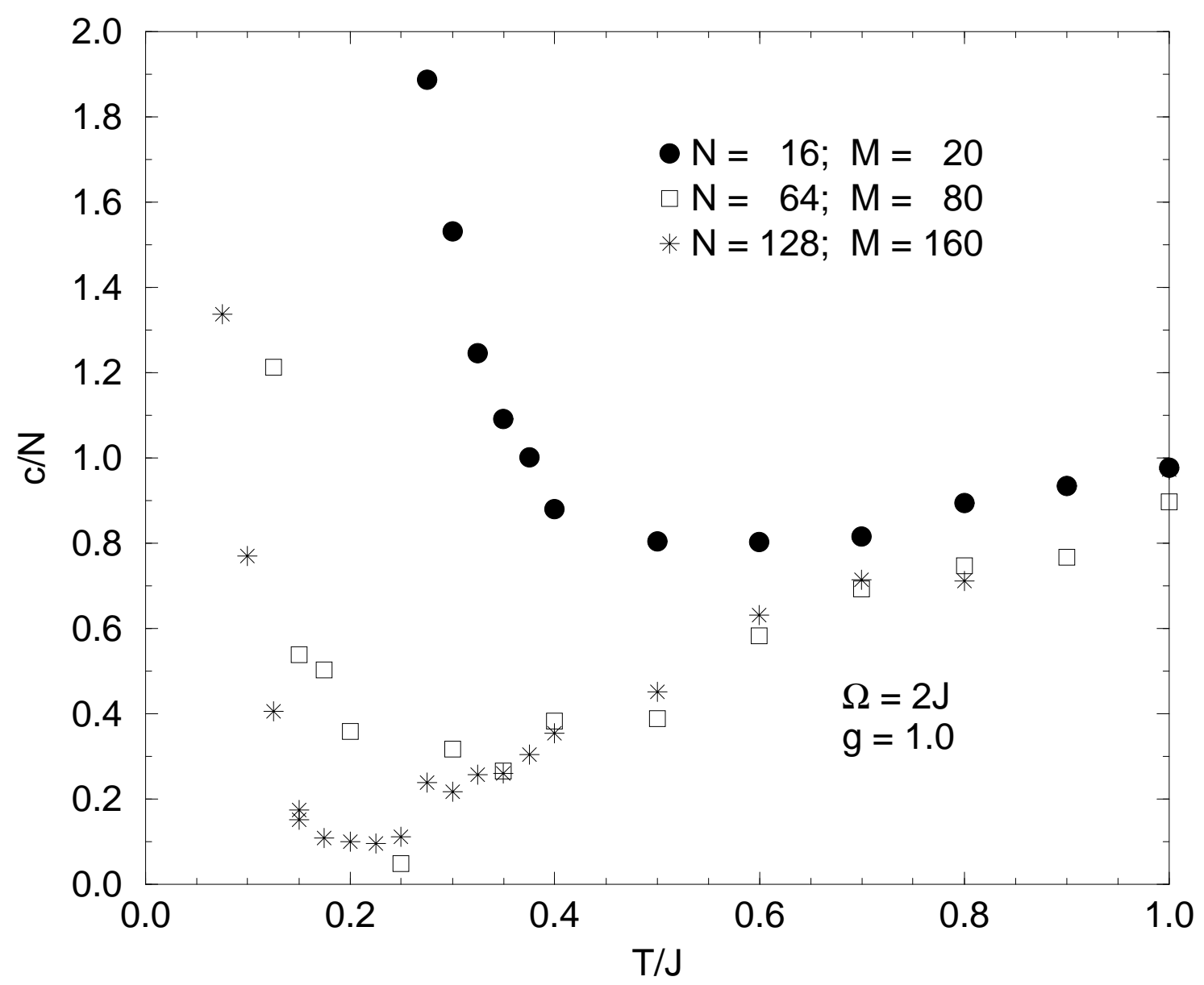

Figure 4.14: Specific heat for a chain with phonon frequency $\Omega=2 J$ and spin-phonon coupling constant $g=1.0$. The steep drop for very low temperatures is a finite-size effect.

The specific heat is an observable which can be as easily measured and calculated as the magnetic susceptibility. However, only for substances with very small spin-spin coupling, such as $\mathrm{Cu}$ (L-alanine) $)_{2}$ with $J=0.55 K[1,2]$, the specific heat can be directly compared with experimental data. For most of the inorganic substances (in particular $\mathrm{CuGeO}_{3}$ with 


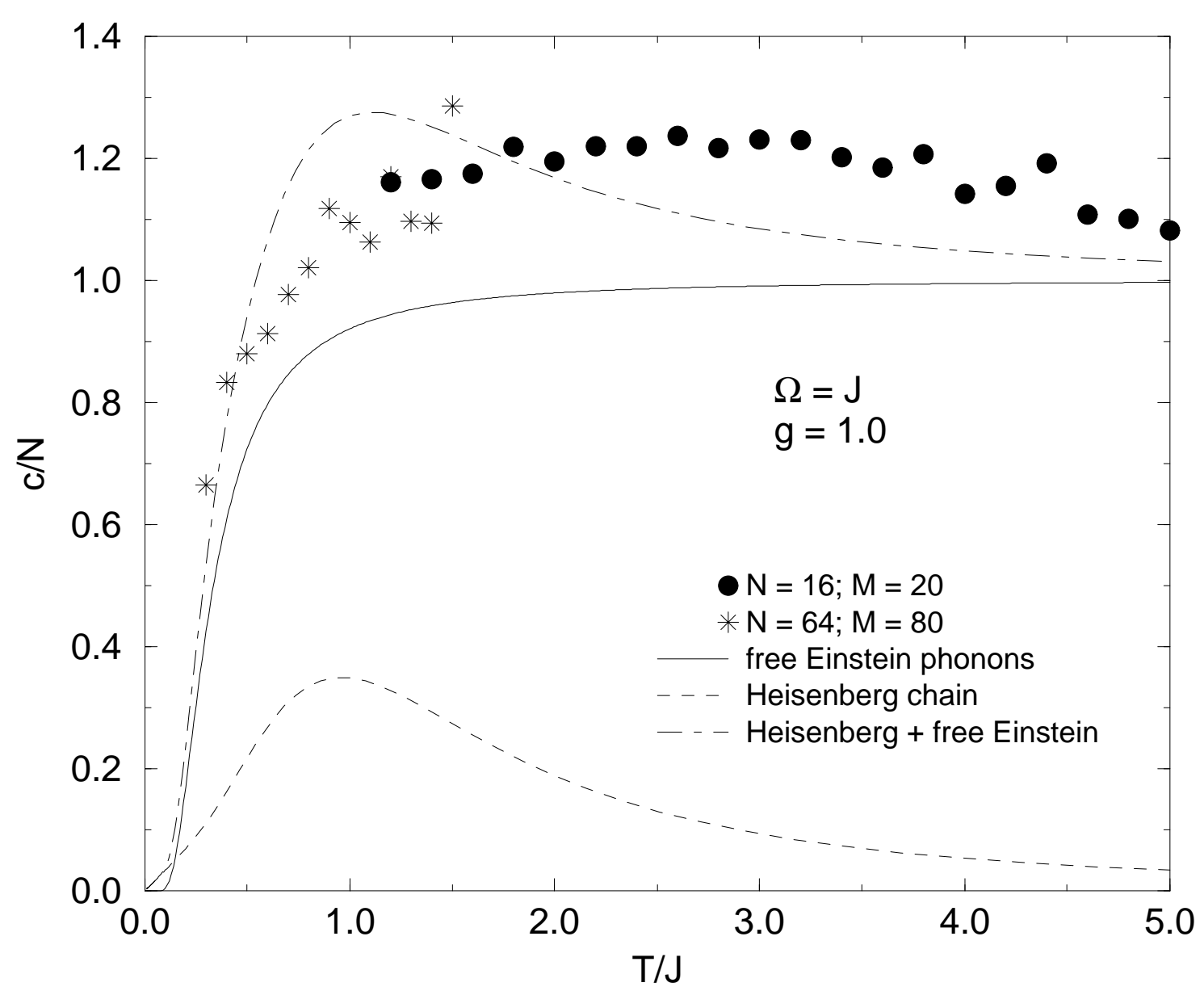

Figure 4.15: Temperature-dependence of the specific heat for a chain with phonon frequency $\Omega=J$ and spin-phonon coupling constant $g=1.0$. The number of sites is 16 and 64 , respectively.

$J \sim 100 K$ ), lattice vibrations dominate the specific heat and it is an unsolved problem to extract its magnetic part. As a first approximation [109] it was assumed for $\mathrm{CuGeO}_{3}$ that it is sufficient to subtract the contribution of the phonons and consider the rest as the contribution of the spins. We show this approximation to be not justified even in the simple case of Einstein phonons. The contribution of the spin-phonon coupling must not be neglected.

In Fig. 4.14 we show the finite-size behavior of the specific heat for a chain with phonon frequency $\Omega=2 J$ and spin-phonon coupling constant $g=1.0$. For given chain length $N$ the correct behavior of the specific heat cannot be traced back to the low temperatures as was possible for the magnetic susceptibility, compare Fig. 3.11. This means, to examine the behavior of the specific heat of the system at a given temperature we have to use a larger chain length than required for the examination of the magnetic susceptibility. 


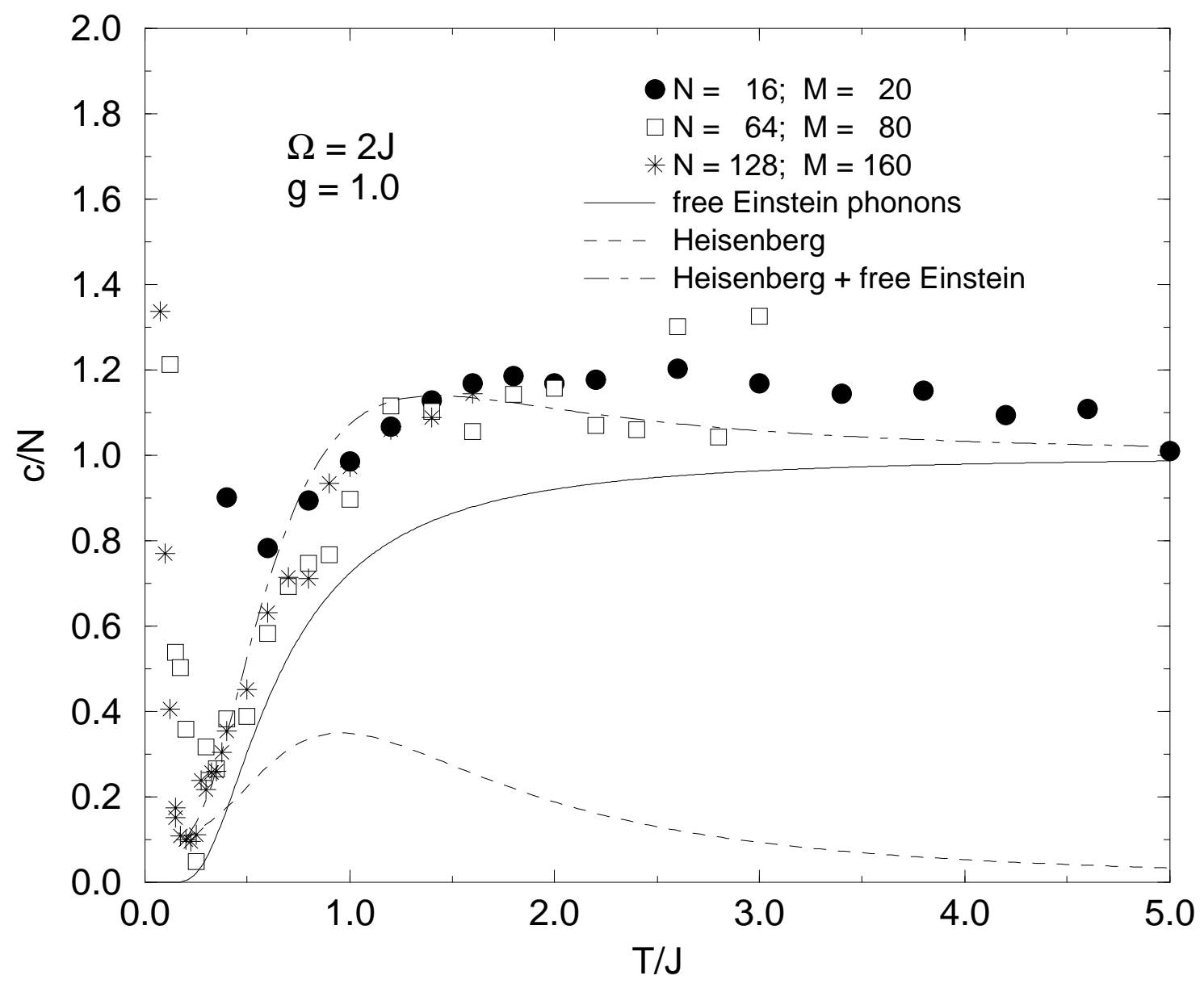

Figure 4.16: Temperature-dependence of the specific heat for a chain with phonon frequency $\Omega=2 J$ and spin-phonon coupling constant $g=1.0$. The number of sites is 16,64 and 128 , respectively.

Furthermore, as can be seen in Figs. 4.15 - 4.17, the statistical fluctuations are much greater for the specific heat than for the magnetic susceptibility.

To demonstrate that the contribution of the spin-phonon coupling may not be neglected, we plot in Fig. 4.15 the specific heat $c$ for a chain with $\Omega=J$ and $g=1.0$ together with the specific heat $c_{f}$ of the free Einstein phonons,

$$
c_{f}=\left(\frac{\Omega}{T}\right)^{2} \frac{\mathrm{e}^{\Omega / T}}{\left(\mathrm{e}^{\Omega / T}-1\right)^{2}},
$$

with $\Omega=J$ and the contribution $c_{H}$ of the isotropic Heisenberg model $[18,19]$. The dominance of the Einstein phonons at high temperatures can clearly be seen. In particular the constancy of the specific heat, as expected according to the Dulong-Petit rule, is visible. Furthermore, the effect of the spin-phonon coupling can be perceived. It shifts the 


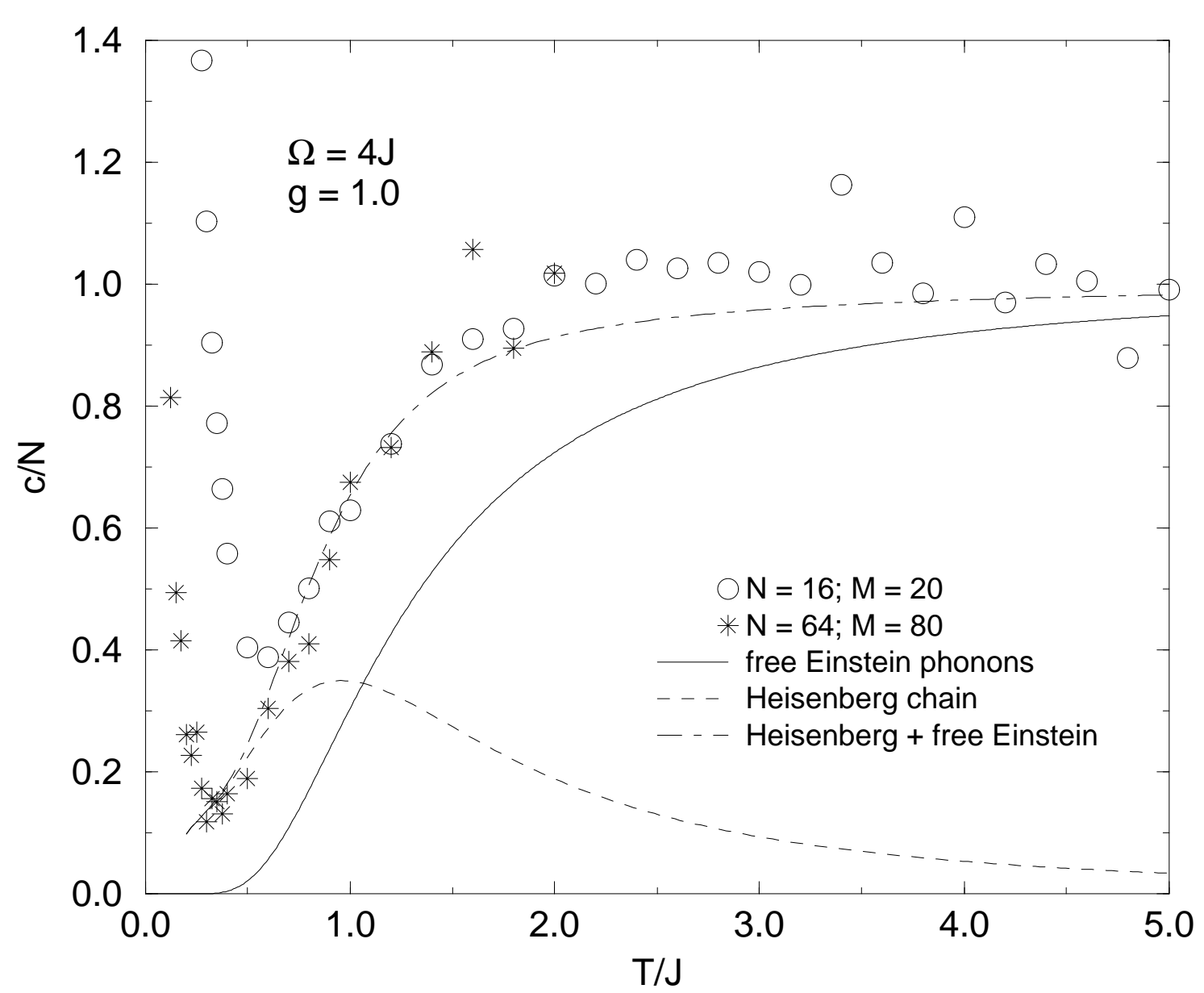

Figure 4.17: Temperature-dependence of the specific heat for a chain with phonon frequency $\Omega=4 J$ and spin-phonon coupling constant $g=1.0$. The number of sites is 16 and 64 , respectively.

maximum at $T \sim J$ of the $c_{f}(T)+c_{H}(T)$ (dot-dashed line) towards higher temperatures, where the maximum becomes less pronounced.

When going to larger frequencies (and $g$ fixed), see Figs. 4.16 and 4.17, the contribution of the spin-phonon coupling decreases. We find that $\Omega>4 J$ is required, so that $c(T)$ can be approximated by $c_{f}(T)+c_{H}(T)$.

\subsection{Local displacement}

Let us recall the definition of the local phonon displacement observable

$$
\delta_{l}=g\left\langle b_{l}^{\dagger}+b_{l}\right\rangle
$$




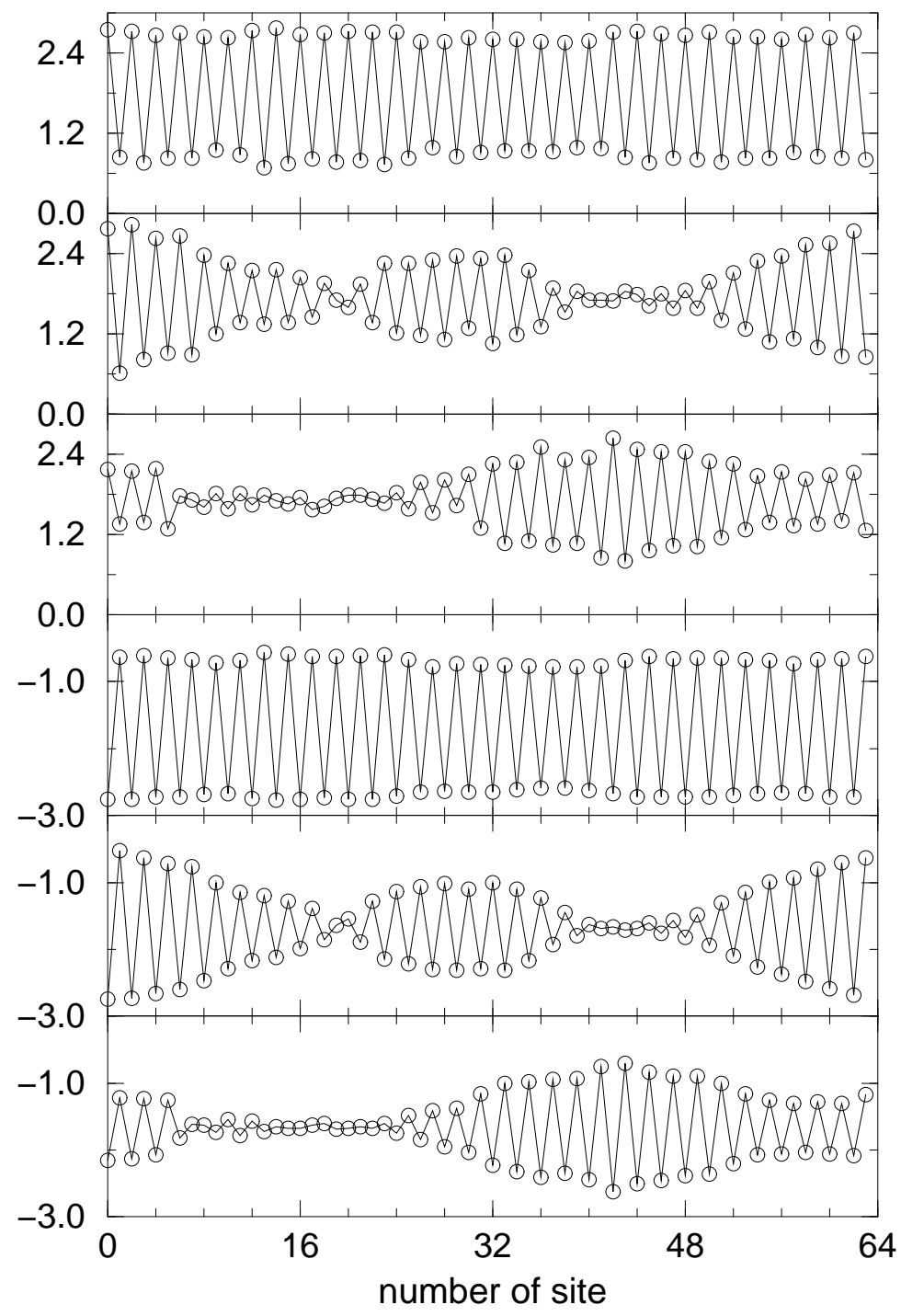

Figure 4.18: Upper three graphs: local displacement $\left\langle b_{l}^{\dagger}+b_{l}\right\rangle$ for $T=0.3 J, 0.4 J, 0.5 J$. Lower three graphs: spin-spin-correlation $\left\langle\vec{\sigma}_{l} \cdot \vec{\sigma}_{l+1}\right\rangle$ for the same temperatures. All graphs are for $N=64, M=80, \Omega=2 J, g=1.5$, and averaged over 10,000 spin updates. Hence, these are snapshots rather than mean values of a thermalized system.

which corresponds in the adiabatic limit to the dimerization in a Heisenberg model with alternating couplings. Its average over all sites $l$,

$$
\delta=\frac{1}{N} \sum_{l=1}^{N} \delta_{l},
$$




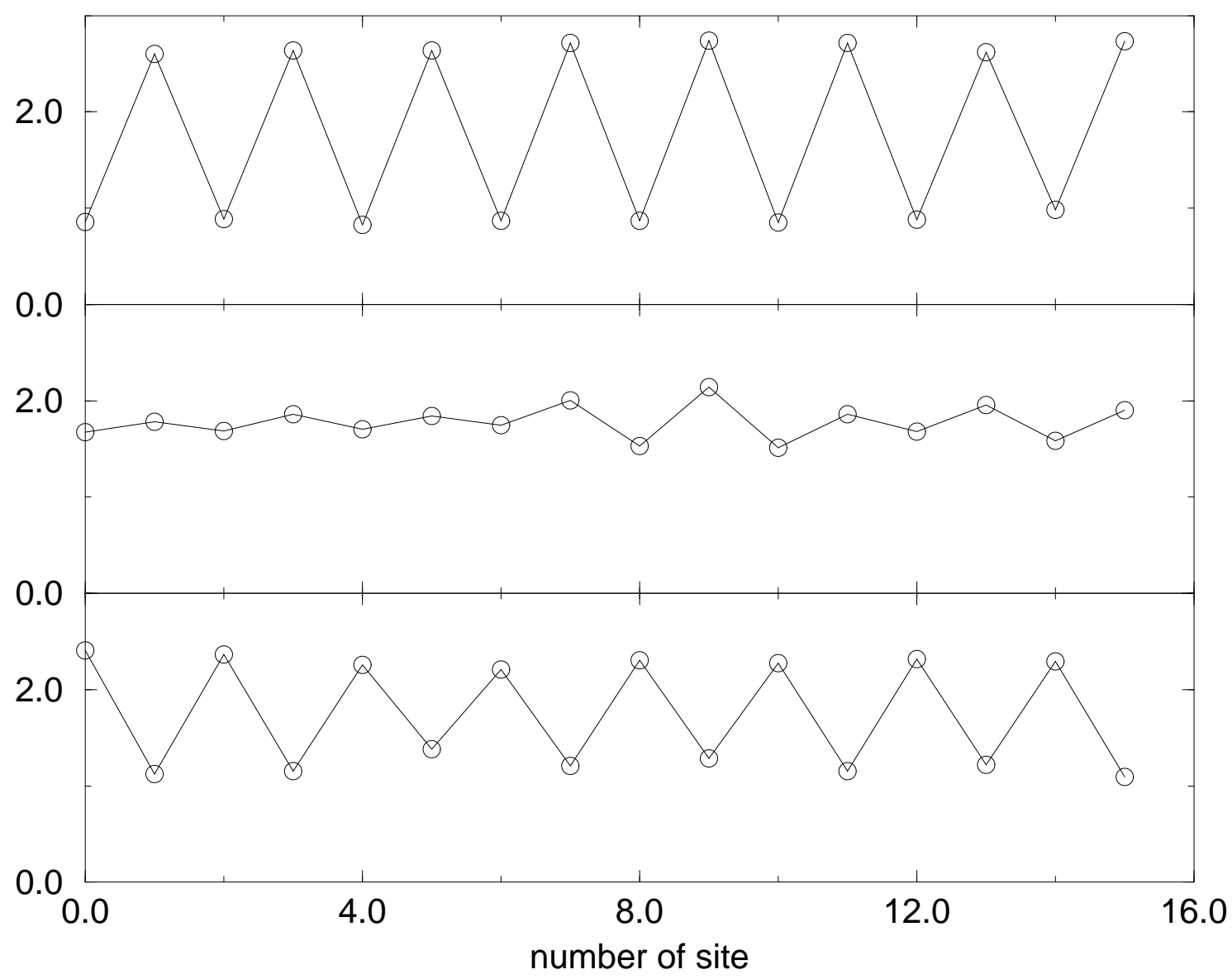

Figure 4.19: Local dimerization $\left\langle b_{l}^{\dagger}+b_{l}\right\rangle$ for a chain with $N=16$ sites, phonon frequency $\Omega=2 J$, and spin-phonon coupling $g=1.5$ at temperature $T=0.5 J$. The successive plots show means over 4000 configurations each, i.e. snapshots. The transition between the two ground states is clearly visible.

represents the uniform phonon displacement.

It is well known that in spin-Peierls substances like $\mathrm{CuGeO}_{3}$ the transition from the uniform to the dimerized phase occurs at finite temperatures. By contrast, here we consider a strictly one-dimensional model with a dimerization transition at $T=0$. To encounter dimerization one therefore has to go to temperatures as low as possible.

It is of interest to see how the periodic patterns in the local phonon displacement, $\left\langle b_{l}^{\dagger}+b_{l}\right\rangle$, are connected with the formation of spin dimers. By calculating the local spinspin correlations, $\left\langle\vec{\sigma}_{l} \cdot \vec{\sigma}_{l+1}\right\rangle$, we find, see Fig. 4.18 , the periodic patterns in the local displacement $\delta_{l}$ to be well in phase with the formation of spin singlet states. This clearly demonstrates the coupling between the phononic degrees of freedom and the spin degrees of freedom. This is also an a posteriori check of the consistency of our combined spin and phonon updates. 
As can be seen from Fig. 4.18 the dimerization is not complete, since the singlet state, $\left\langle\vec{\sigma}_{l} \cdot \vec{\sigma}_{l+1}\right\rangle=-3.0$, is not exactly reached and the correlation between the dimers is not zero.

Furthermore, we see from this figure how the system crosses over from the dimerized state at $T=0.3 J$ to the disordered state for $T>0.5 \mathrm{~J}$. The appearance of domain walls between dimerized regions is clearly seen at $T=0.4 \mathrm{~J}$.

The behavior of the magnetic susceptibility for this system is presented in Fig. 4.1. For $T \leq 0.3 J$ the susceptibility is close to zero, because of the spin gap. Between $T=0.3 J$ and $T=0.5 J$ the susceptibility grows rapidly. At higher temperatures, the effects of the spin gap are negligible and the susceptibility can be described by that of the Heisenberg chain with a rescaled spin-spin coupling, cf. Fig. 4.7. This behavior shows that the spin gap of the magnetic susceptibility and the incomplete dimerization occur simultaneously. Domain walls appear at temperatures where the susceptibility rises.

In the dimerized state transitions between the two ground states can occur. This can be inferred from Fig. 4.19, where the successive plots show means over 4000 configurations.

\subsection{Phase diagram}

The Heisenberg chain coupled to (Einstein) phonons is expected to show a transition from the gapless phase to the gapped phase. The Heisenberg chain is known to be gapless. The transition is expected to occur at a finite critical spin-phonon coupling $g_{c}(\Omega)$ which depends on the phonon frequency. This behavior has already been found by Sandvik and Campbell [9], Weisse et al. [97] and Raas and Uhrig [99] for the model with phonons localized on the bonds. Bursill et al. [8] have presented a phase-diagram with finite $g_{c}(\Omega)$ for the model with phonons localized on the sites.

The local phonon displacement turned out to be a valuable tool to decide, for fixed parameter values, whether the model is in the dimerized state.

We used this property to determine the approximate phase boundaries in the $g-\Omega$ plane. To be more precise, we calculated $\delta_{l}$ for a 64 site system (with $M=80$ ) at temperatures $T / J=0.1, \ldots, 0.4$ and inferred from the periodic fluctuations the phase diagram shown in Fig. 4.20. For all points $\delta_{l}$ either shows random patterns or the periodic fluctuations over the entire chain. Hence, we can determine whether the model at a certain point in parameter space is in the dimerized phase.

Since we are limited both in system size and temperature, the location of the separation line between the two phases is obtained for a finite chain with size $N=64$. Since we cannot decide whether or not dimerization will appear for $T<0.1 J$, the actual separation line will be shifted to the left. Indeed, we examined the model of Ref. [7] $(g=1 /(4 \sqrt{2})$ and $\Omega=0.2 J)$ with $N=256$ and $M=320$ and found dimerization for temperatures $T \leq 0.06 J$. This is in accordance with the claim by Sandvik et al. [7] who argue that this model is dimerized at zero temperature. Hence, the apparent constancy of the critical coupling constant $g_{c}$ for $\Omega \leq J$ is in reality an artifact of the finite temperature.

Our phase diagram for $J \leq \Omega \leq 3 J$ has been confirmed by a recent investigation by Raas and Uhrig [99]. Their investigation confirms also our result for the $N=256$ chains 


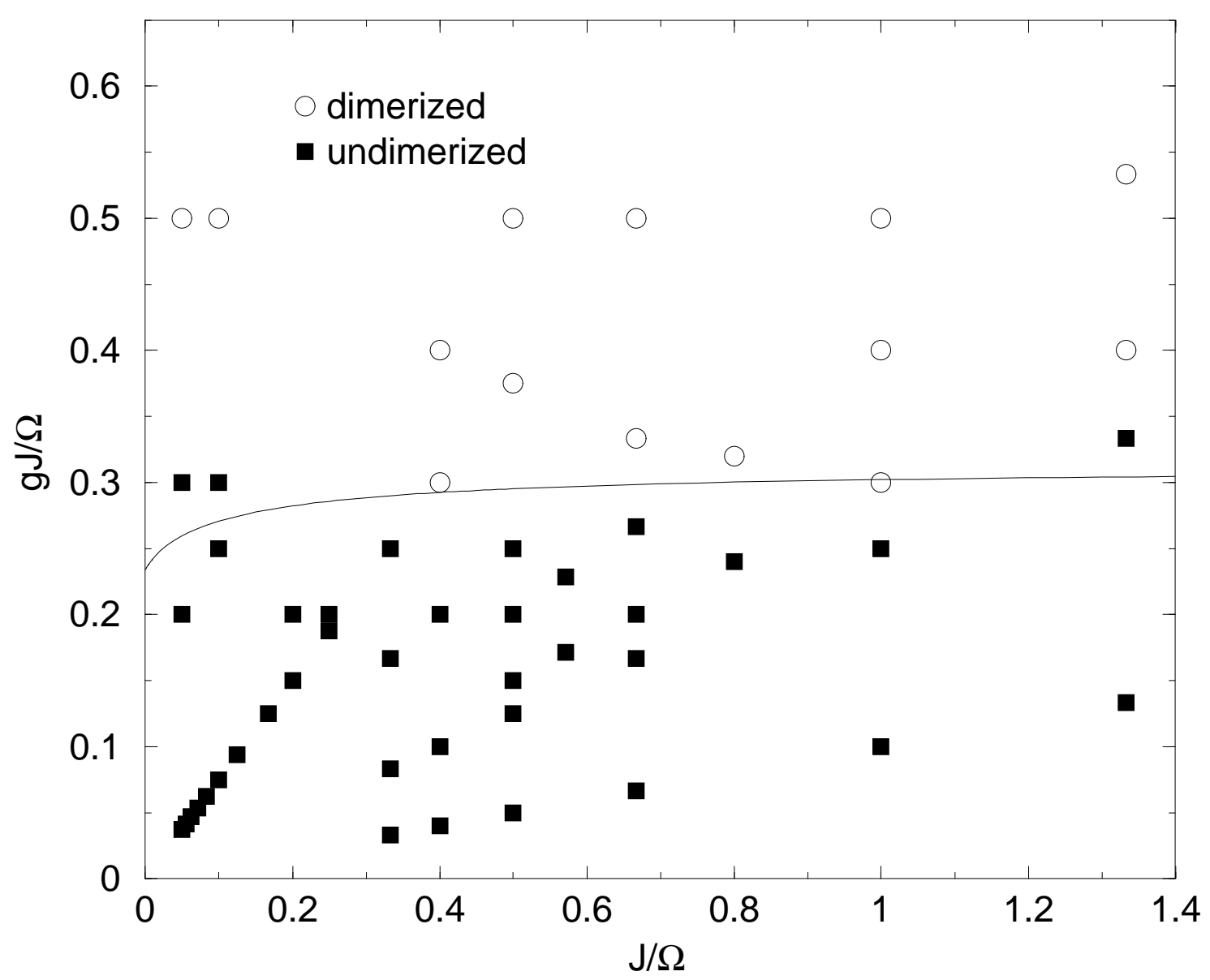

Figure 4.20: Phase-diagram of the model in the $J / \Omega-g J / \Omega$ plain. Circles (squares) mark points with (without) dimerization. The solid line shows the phase boundary of Ref. [99].

at higher frequencies. Both our result and that of Raas and Uhrig differ from that of Weisse et al. [97].

Furthermore, the $g_{c}(\Omega)$ for this model is roughly twice that of the model considered by Bursill et al. [8] in the frequency range $J \leq \Omega \leq 3 J$.

\subsection{Correlation function}

The investigation of the temperature dependence of the spin-spin correlation length is important for the understanding of the critical behavior of the examined system. Neutron scattering experiments allowed its determination for the two-dimensional antiferromagnetic Heisenberg material $\mathrm{Sr}_{2} \mathrm{CuO}_{2} \mathrm{Cl}_{2}$ in the temperature range $0.19 \leq T / J \leq 0.4$, where the spin-spin coupling constant is $J=125 \pm 6 \mathrm{meV}[110,111]$.

$\mathrm{X}$-ray diffuse scattering measurements in $\mathrm{CuGeO}_{3}$ in the temperature range $14 \mathrm{~K} \leq$ 


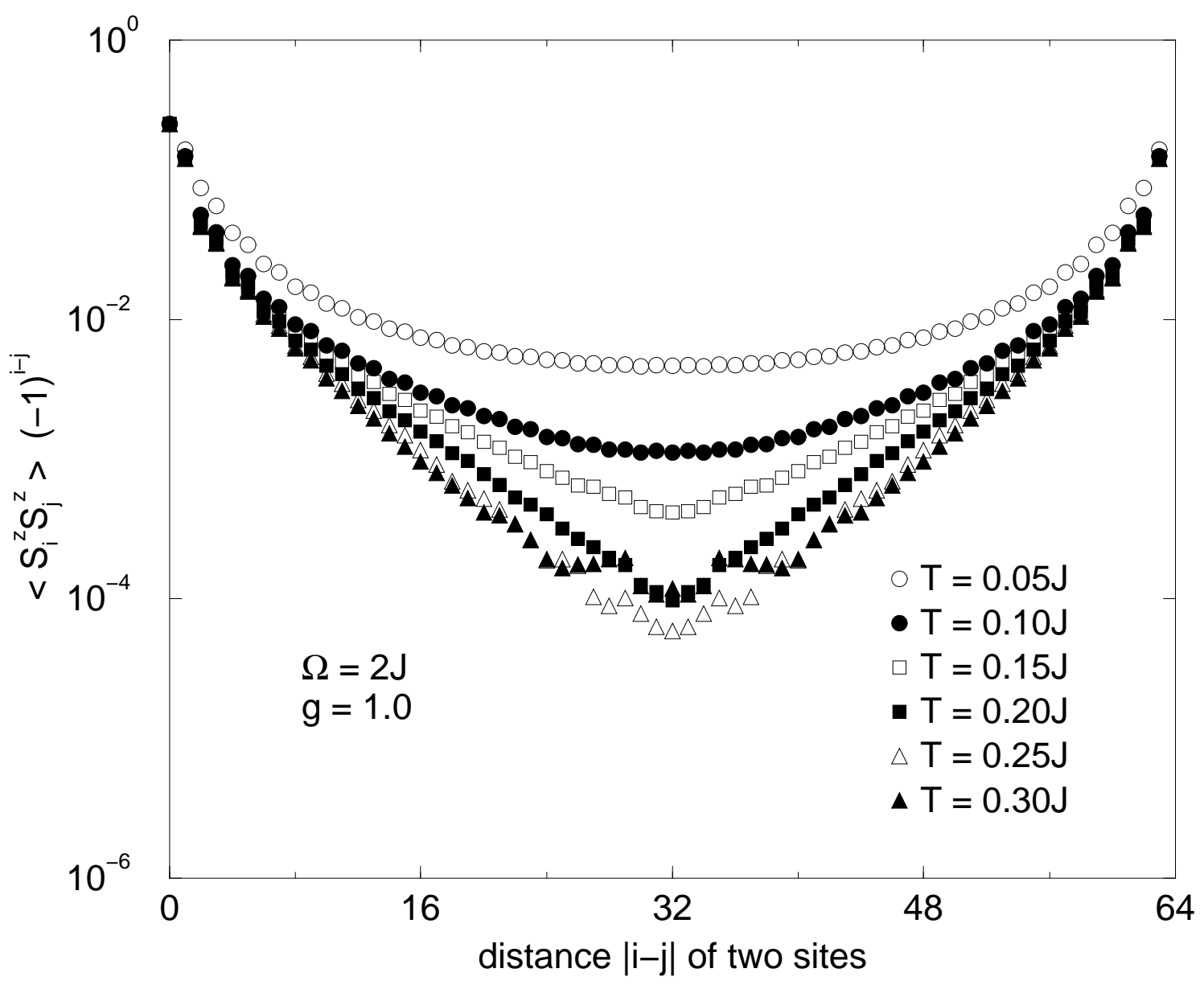

Figure 4.21: Temperature dependence of the staggered spin-spin correlation function $\left\langle S_{i}^{z} S_{j}^{z}\right\rangle(-1)^{i-j}$ for a chain with $\Omega=2 J, g=1.0, N=64$, and $M=80$. Statistical fluctuations are of the order $10^{-4}$. For this chain, as well as to all the other chains we considered, the staggered correlation function can be fitted by a function proportional to $\exp (-N /(2 \xi)) \cosh ((N / 2-r) / \xi)$, where $\xi$ is the correlation length (in units of the lattice constant $a$ ). Only two conditions have to be satisfied, the mutual distance $r$ of two sites has to be larger than $\xi$ and finite size effects have to be negligible. This is satisfied for $N \geq 10 \xi$.

$T \leq 40 \mathrm{~K}$ have shown that the inverse correlation length in the $c$-direction behaves as $\sqrt{T-T_{s p}}$, where $T_{s p}=14 \mathrm{~K}$ is the critical temperature of the spin-Peierls phase transition [94]. The corresponding critical exponent is $\nu=1 / 2$.

The critical exponent of the isotropic Heisenberg chain is $\nu=1$. In contrast to real materials, however, the critical temperature is zero.

With our method it is straightforward to calculate the staggered spin-spin correlation function $\left\langle S_{i}^{z} S_{j}^{z}\right\rangle(-1)^{i-j}$ for the model given by Eq. (3.1). In the following we will examine 


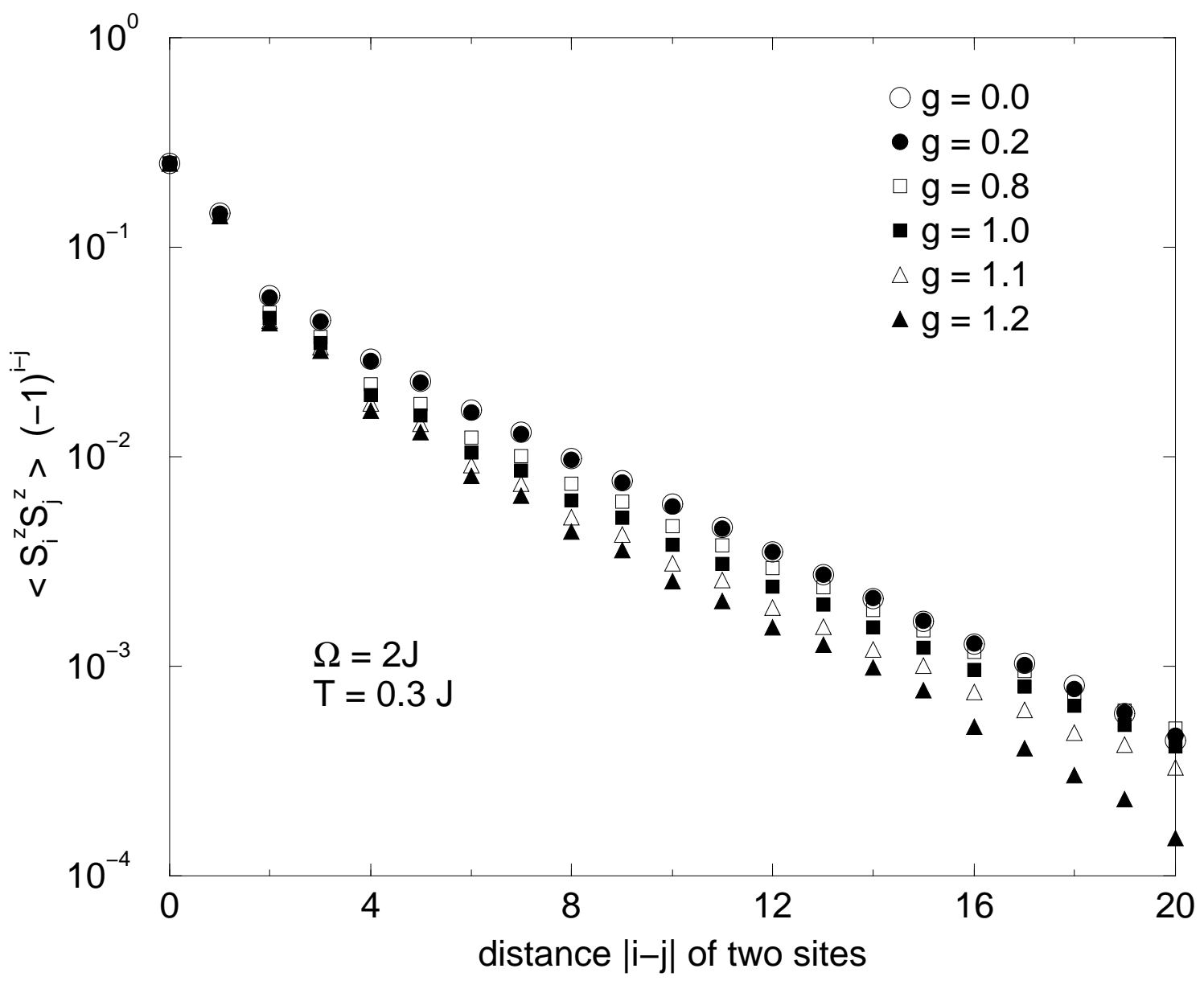

Figure 4.22: Staggered spin-spin correlation function $\left\langle S_{i}^{z} S_{j}^{z}\right\rangle(-1)^{i-j}$ as a function of the distance $|i-j|$ of two sites for a chain with $\Omega=2 J, N=64$, and $M=80$ at the low temperature $T=0.3 J$. The spin-phonon coupling $g$ is varied between 0.0 and 1.2.

it as a function of temperature, phonon frequency, and spin-phonon coupling. Our results can be summarized as follows.

(i) The correlation function decreases with growing temperature. This is shown for $\Omega=2 J$ and $g=1.0$ in Fig. 4.21 (see also Fig. 4.28 for $\Omega=2 J$ and $g=0.5$ ). This decrement is expected, because finite temperature effects disturb spin-spin correlations.

(ii) For increasing phonon frequency $\Omega$ at fixed $g$ one finds the correlations more and more unaffected by the spin phonon coupling. This results from the decoupling of the magnetic and phononic degrees of freedom, as we observed before when studying the magnetic susceptibility.

(iii) As a function of $g$ at fixed $\Omega$ (and also as a function of $\Omega$ at fixed $g$ ) we observe a crossover between the low-temperature and the high-temperature range. At low temperatures the correlations (and the correlation lengths) decrease either with growing spin-phonon coupling (Fig. 4.22), or with decreasing phonon frequency, respectively 


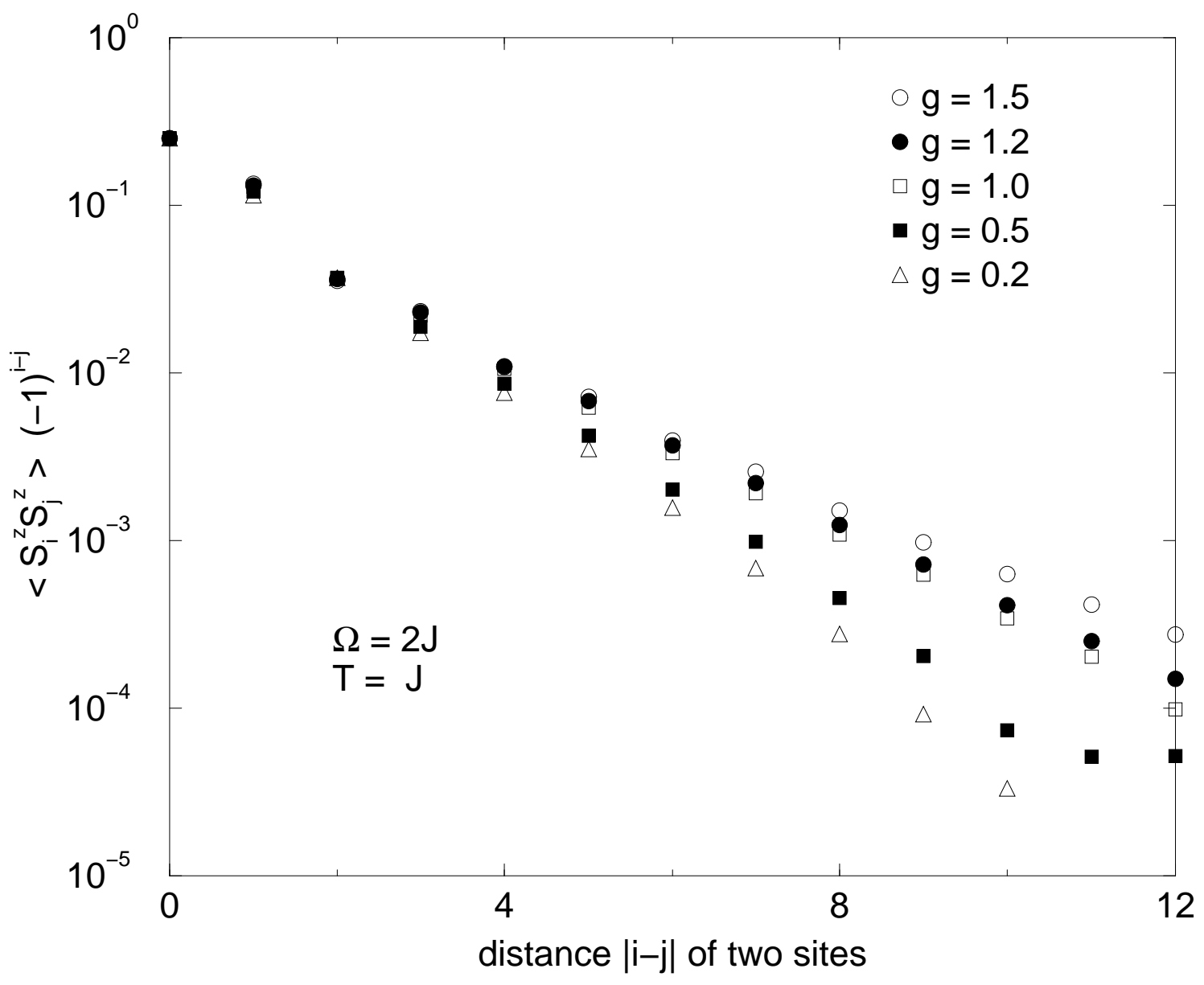

Figure 4.23: Staggered spin-spin correlation function $\left\langle S_{i}^{z} S_{j}^{z}\right\rangle(-1)^{i-j}$ as a function of the distance $|i-j|$ of two sites for a chain with $\Omega=2 J, N=64$, and $M=80$ at the high temperature $T=J$. The spin-phonon coupling $g$ is varied between 0.2 and 1.5.

(Fig. 4.24). This is analogous to the dimerized model where one finds a lowering of the correlation with growing dimerization. For high temperature, however, the inverse behavior occurs. The spin-spin correlations (and the correlation lengths) increase with growing $g$ and decreasing $\Omega$ (Figs. 4.23, 4.25). The transition between the low and the high-temperature region occurs at $T=(0.55 \pm 0.05) J$ for $\Omega=2 J$. We will explain this behavior in the next section where we examine the temperature dependence of the correlation length as a function of $g$ and $\Omega$.

(iv) For sufficiently large spin-phonon coupling $g(\Omega)$, the spin-spin correlation function as a function of distance shows a pronounced steplike behavior at low temperatures (see Fig. 4.24). This is a signature of the dimerization of the ground state. We find a finite correlation between the dimers, whereas a completely dimerized state would have one step only. This incomplete dimerization results from finite temperature effects.

(v) The correlation length $\xi$ in units of the lattice constant $a$ as a function of inverse 


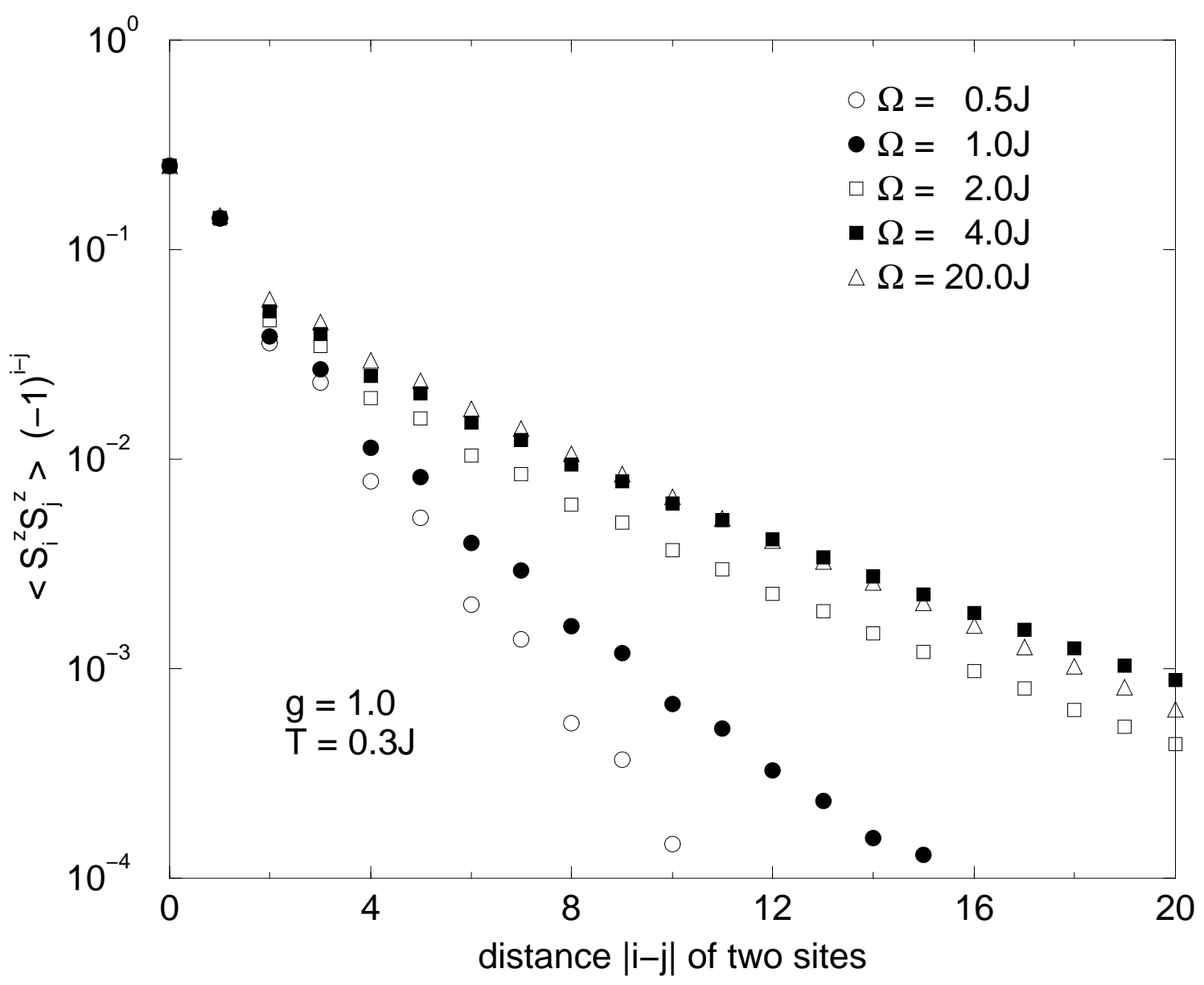

Figure 4.24: Staggered spin-spin correlation function $\left\langle S_{i}^{z} S_{j}^{z}\right\rangle(-1)^{i-j}$ as a function of the distance $|i-j|$ of two sites for a chain with $g=1.0, N=64$, and $M=80$ at the low temperature $T=0.3 J$. The phonon frequency $\Omega$ is varied between $0.5 J$ and $20 J$. The steps with the width of two lattice sites for $\Omega=0.5 J$ and $\Omega=J$ result from the incomplete dimerization of the chain.

temperature, inverse phonon frequency, and spin-phonon coupling is shown in Fig. 4.26. The exact dependence $\xi(g, \Omega, T)$ will be examined in detail for the gapless phase in the following chapter.

(vi) The pronounced maxima of the static structure factor $S(q, T)$ at momentum $q=\pi$ for $g=0$ as shown in Fig. 4.27 originate from the logarithmic divergence of $S(q, T=0)$ of the isotropic Heisenberg model. (For a study as to what extent this divergence can be seen at finite $T$ we refer to Ref. [112].) For the model in the gapless phase the peak in $S(q)$ at $q=\pi$ is only slightly diminished as compared to the Heisenberg model, indicating that the models show a similar divergent behavior. On the contrary, in the gapped phase the structure factor shows a broad maximum hinting at a regular behavior of $S(q=\pi, T=0)$.

In the following section where we examine the temperature dependence of the spin- 


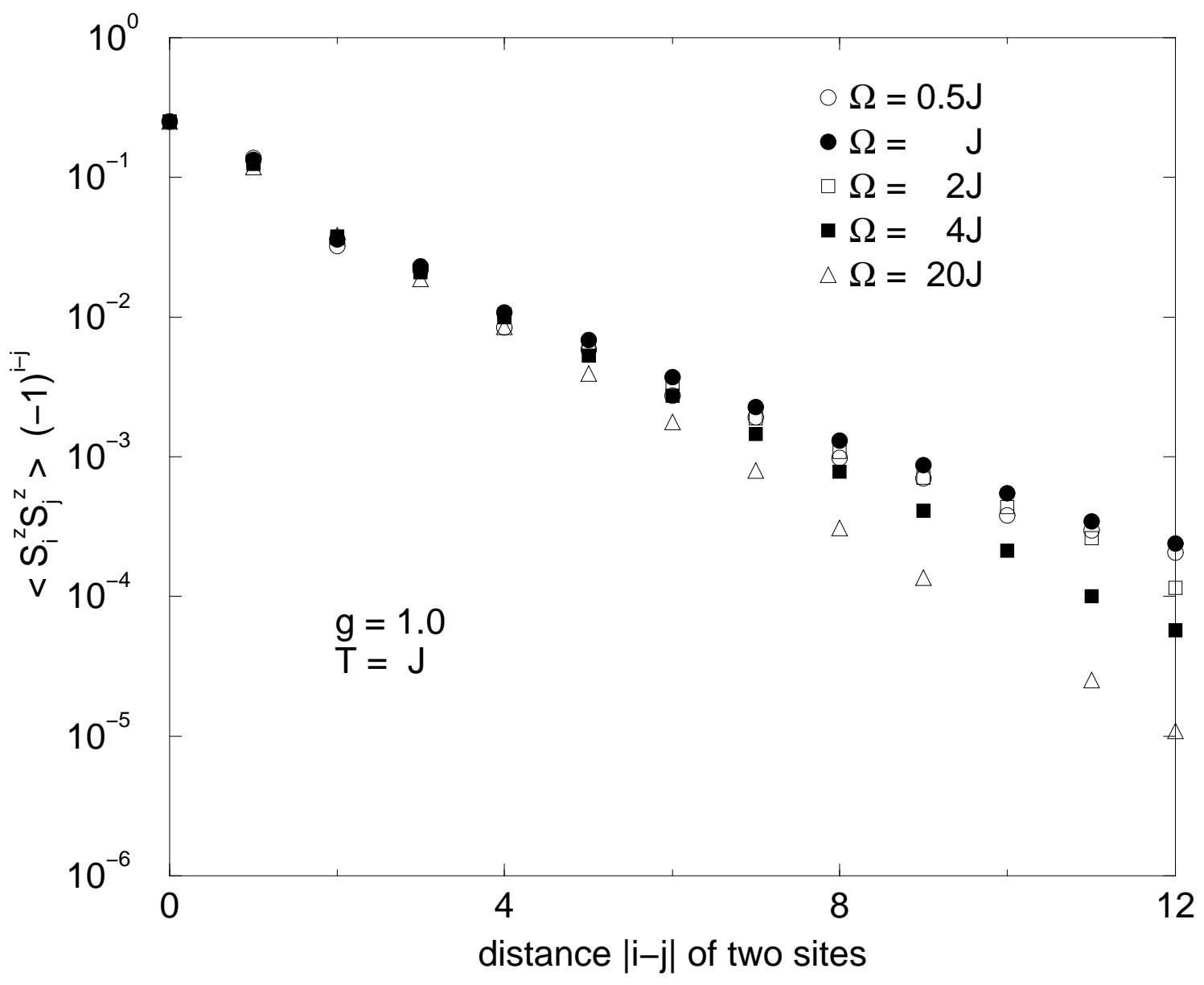

Figure 4.25: Staggered spin-spin correlation function $\left\langle S_{i}^{z} S_{j}^{z}\right\rangle(-1)^{i-j}$ as a function of the distance $|i-j|$ of two sites for a chain with $g=1.0, N=64$, and $M=80$ at the high temperature $T=J$. The phonon frequency $\Omega$ is varied between $0.5 J$ and $20 J$.

spin correlation length $\xi$ on the phonon frequency $\Omega$ and the spin-phonon coupling $g$ we will find,

$$
\frac{\xi}{a}=c_{H}(T)\left(1+\frac{g^{2} J}{\Omega}\right)\left(\frac{T}{J}\right)^{-1+0.5|g|^{3 / 2} J / \Omega},
$$

where $a$ is the lattice constant and $c_{H}(T)$ is a nearly temperature independent factor given by the Heisenberg chain, whose numerical values have been determined with high precision by Klümper [19]. We have to note that we will investigate systems only in the temperature region $0.2 J \leq T \leq J$. Hence, we will show that Eq. (4.25) is a good approximation in this temperature region only. We do not claim that this equation is an approximation for $T \rightarrow 0$ or $T \rightarrow \infty$. Moreover we will confirm this equation only for systems which are either gapless or are in the gapped phase and near the phase boundary.

We present this formula already in this section, because with its help we are able to 

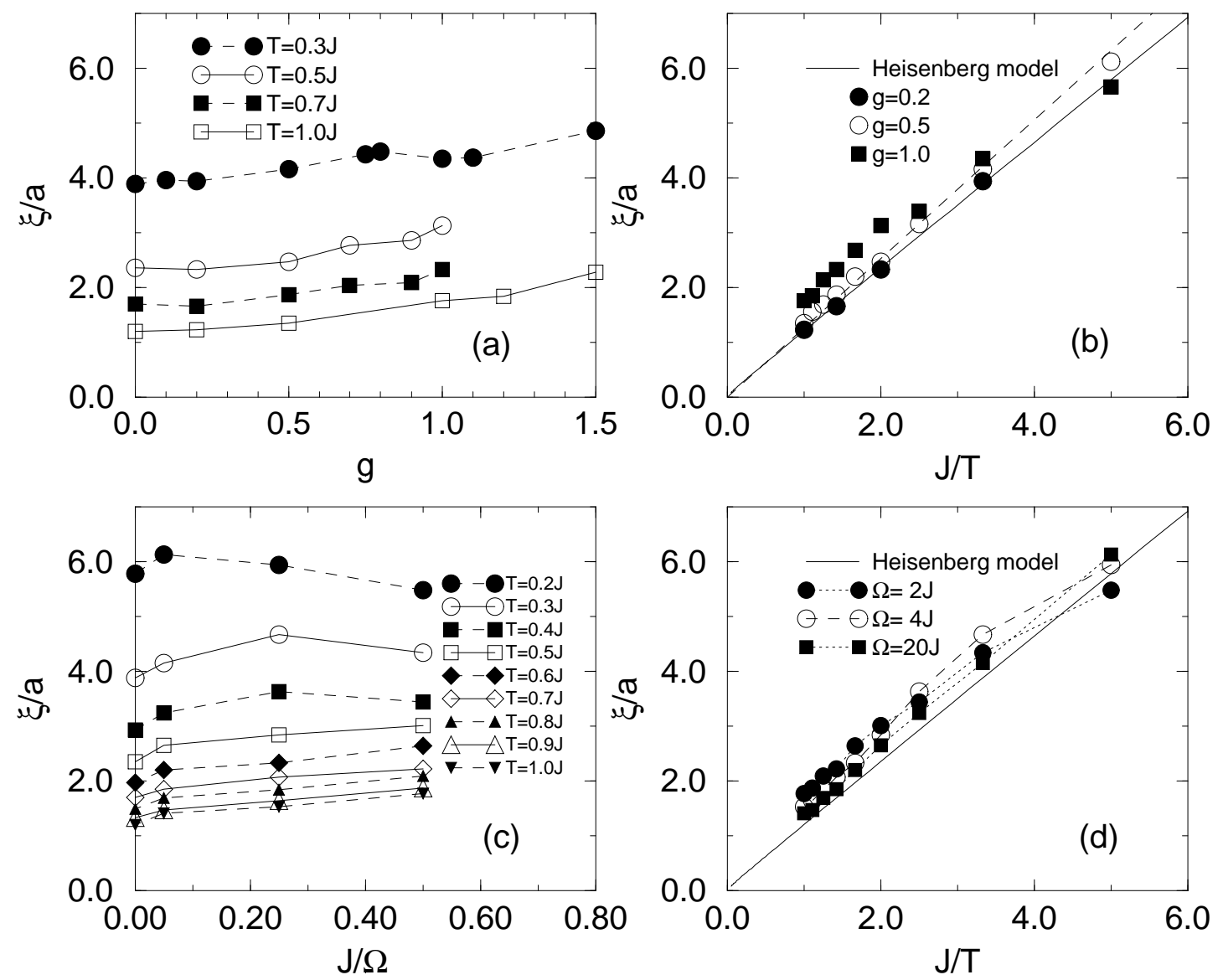

Figure 4.26: Correlation length $\xi$ in lattice units $a$ for $\Omega=2 J$ (upper figures) and $g=1.0$ (lower figures). The chain length is $N=64$ and the Trotter number $M=80$. The quantitative dependence of the correlation length on phonon frequency, spin-phonon coupling, and temperature will be examined in the following section.

understand the following results which we obtained in this section.

At low temperatures, the correlation length increases with growing $\Omega$ (for $T=0.2 J$ this behavior can be seen for $g \geq 0.1$ ) and with decreasing $g$ (for $T=0.2 J$ this behavior can be observed for $\Omega \leq J$ ). This explains the results shown in Figs. 4.22 and 4.24 .

At high temperatures, the correlation length decreases with growing $\Omega$ (for $T=2 J$ this behavior can be seen for $g \geq 0.1$ ) and with decreasing $g$ (for $T=2 J$ this behavior can be observed for all values of $\Omega$, the effect becomes more pronounced the smaller $\Omega$ becomes). This explains the results shown in Figs. 4.23 and 4.25.

Between this low and high temperature region (whose boundaries depend on $g$ and $\Omega$ ) the behavior of $\xi$ is not monotonous when either $g$ or $\Omega$ are varied. This explains the non-monotonous behavior of $\xi$ for $0.2 J \leq T \leq 0.4 J$ shown in Fig. 4.26a. 


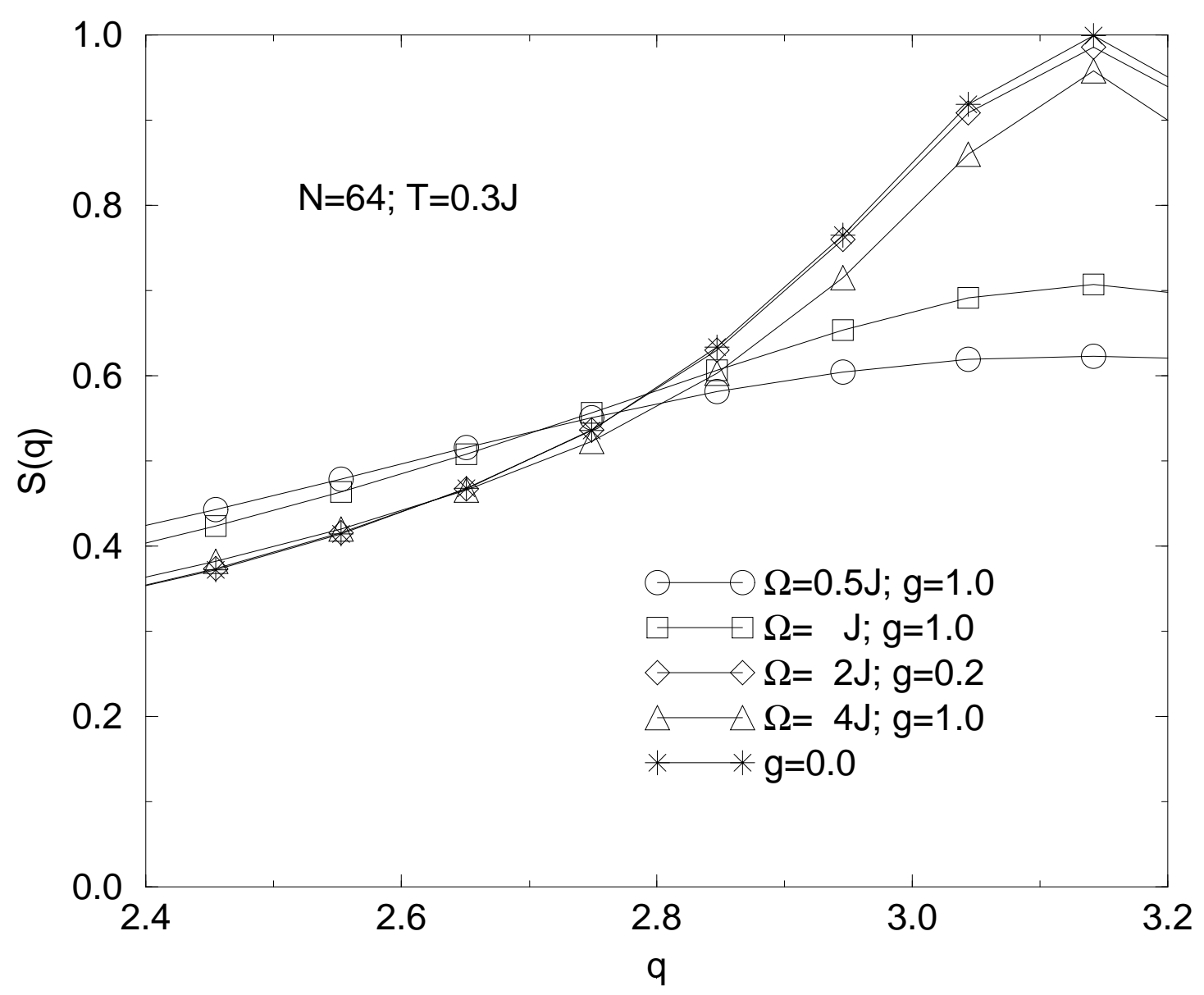

Figure 4.27: Structure factor $S(q)$ versus momentum $q$. The chain length is $N=64$ and the data were extrapolated for Trotter number $M \rightarrow \infty$ by using the data for $M=40$ and $M=80$.

\subsection{Correlation length}

In the following examination we would like to examine the dependence of spin-spin correlation length of the model, Eq. (3.3) on the spin-phonon coupling $g$, the phonon frequency $\Omega$, and the temperature $T$. The investigated temperature region is $0.2 J \leq T \leq J$. We choose the chain length $N=64$ and the Trotter number $M=80$, because for these values finite-size effects are negligible for temperatures $T \geq 0.2 \mathrm{~J}$. For all our runs we find a truncation of 50 phonons per site to be sufficient for the temperature range investigated. The probability for more than 50 phonons per site is less than $10^{-6}$. 


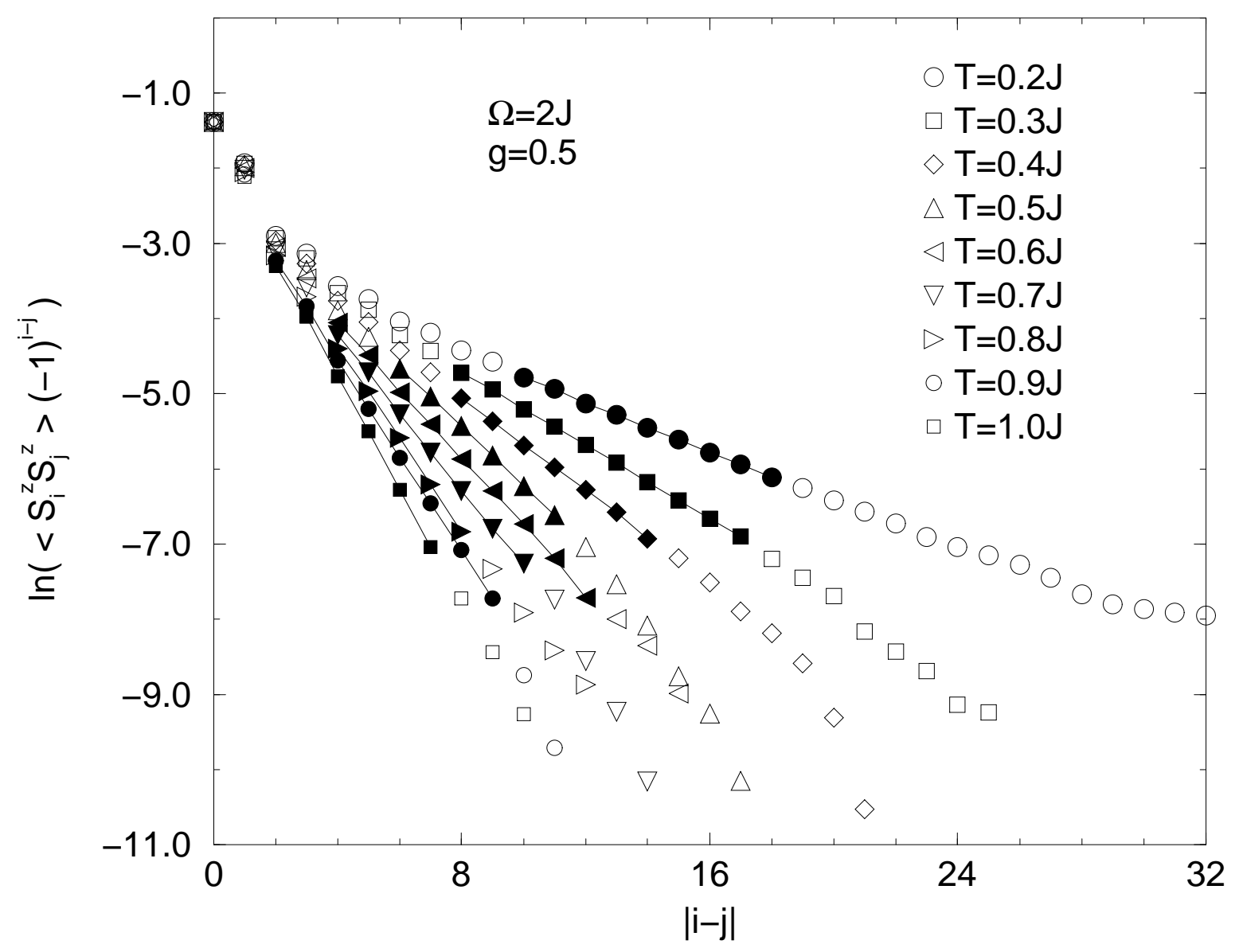

Figure 4.28: Logarithm of the staggered correlation function $\left\langle S_{i}^{z} S_{j}^{z}\right\rangle(-1)^{i-j}$ versus $|i-j|$. Black symbols show the region where the correlation function decays exponentially. The correlation length $\xi$ was determined by using the data points of this region only.

We examine the correlation length $\xi$ of the correlation function $G(r)=\left\langle S_{i}^{z} S_{j}^{z}\right\rangle(-1)^{i-j}$, where $S_{i}^{z}$ is the z-component of the $i$ th spin. In one-dimensional Heisenberg systems this function decays according to $G(r) \propto \exp (-r / \xi)$ for $r \gg \xi$ and $T \neq 0$ (Ref. [113]), where $r=a|i-j|$ and $a$ denotes the lattice constant. Because of statistical fluctuations quantum Monte Carlo simulations allow the examination of this decay only in a finite region of $r$. A typical result is shown in Fig. 4.28. The correlation lengths of all the systems we investigated are $a<\xi<7 a$. Therefore the chain length $N$ is (with few exceptions) at least an order of magnitude larger than $\xi / a$. Hence, finite-size effects are unlikely to alter our results significantly. (For comparison, Ding and Makivić used only $N \geq 6 \xi / a$ 


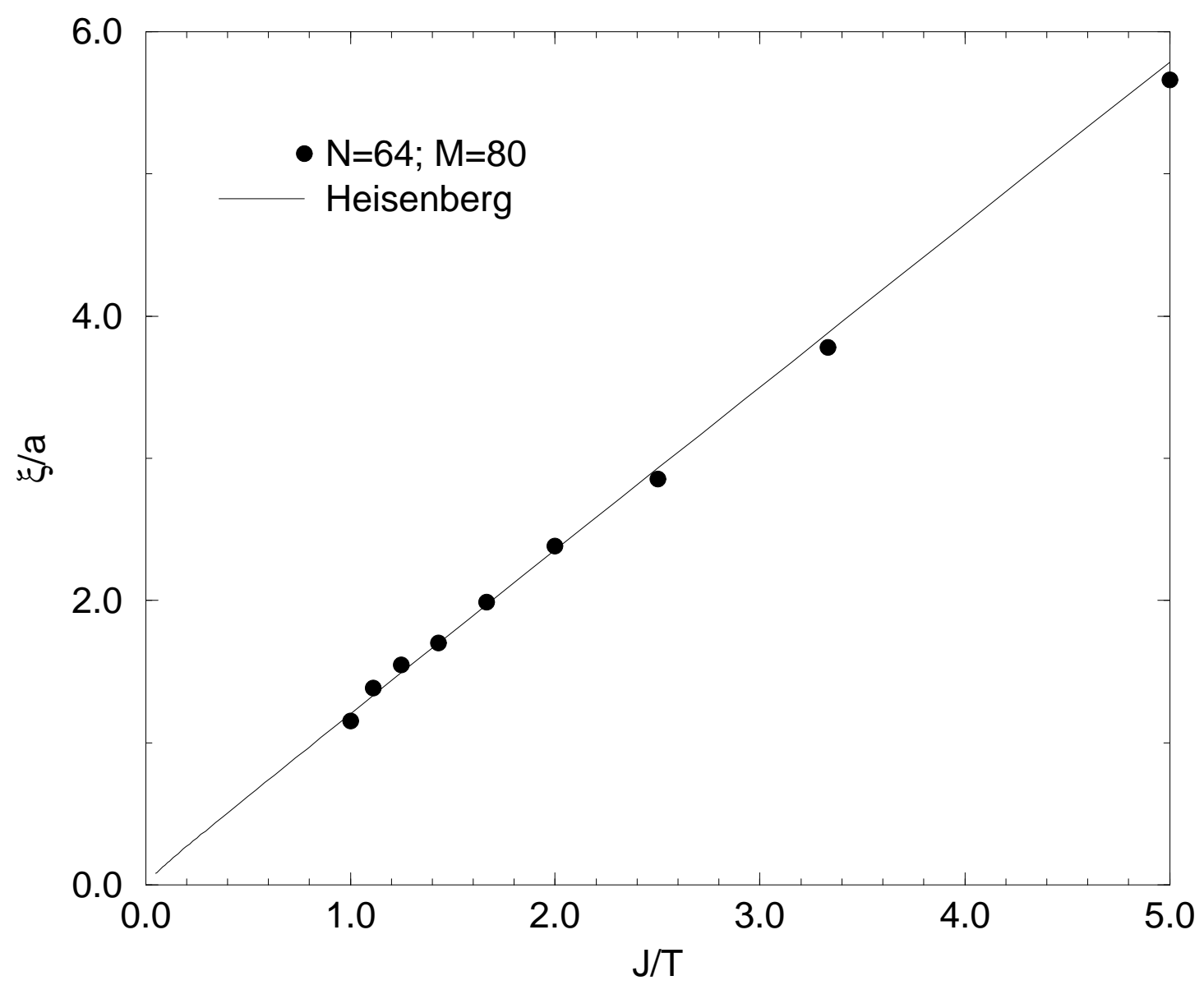

Figure 4.29: Correlation length $\xi$ of the Heisenberg model in units of the lattice constant $a$ as a function of the inverse temperature. The solid line shows the exact data from A. Klümper. The proportionality $\xi \propto T^{-1}$ is confirmed.

[71] and $N \geq 5 \xi / a$ [72], respectively, while stating the finite-size effects to be small. In addition they usually used Trotter numbers less than the lattice size.) Furthermore, a simulation with $N=64$ and $M=80$ for the Heisenberg chain in the temperature range $0.2 J \leq T \leq J$ yielded $\xi$ within an inaccuracy of $\leq 3 \%$, when compared with the exact data of Ref. [19] (see Fig. 4.29). This value sets an upper limit on the effects of finite size and finite Trotter number.

In Fig. 4.30 we present $\xi$ as a function of the inverse temperature. The linear fit for the system with $\Omega=2 J$ and $g=1.0$ is poor.

In the following we will examine the dependence of the correlation length $\xi$ (in units of 

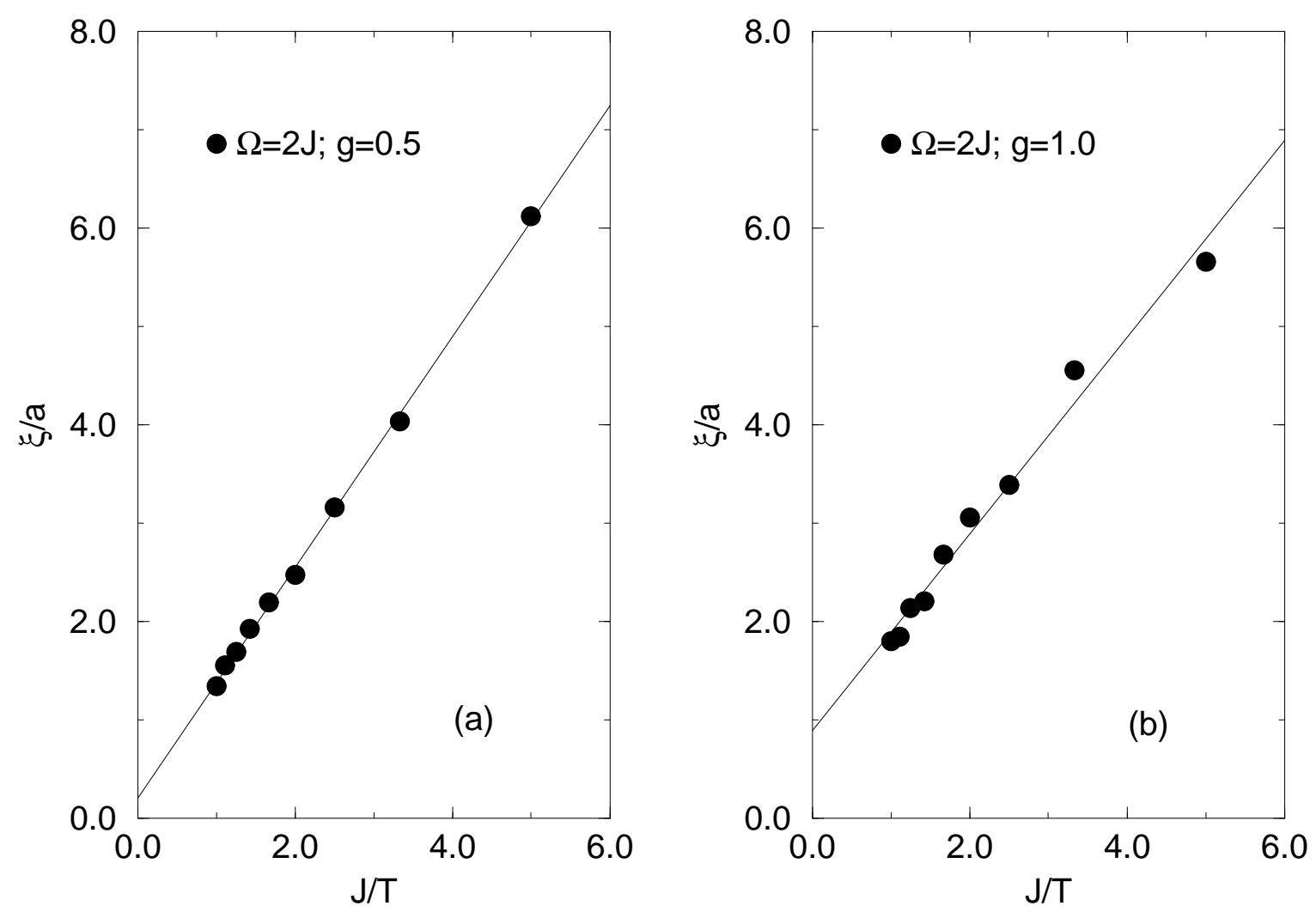

Figure 4.30: Correlation length $\xi$ in units of the lattice constant $a$ as a function of the inverse temperature. The solid line is the linear fit. The data points for $\Omega=2 J$ and $g=1.0$ appear to lie not on a straight line. This inspires us to examine the possibility that the temperature dependence of the correlation length shows a power-law behavior in this temperature region.

the lattice constant $a$ ) on the phonon frequency $\Omega$ and the spin-phonon coupling constant $g$. The formula we would like to determine is $\xi / a=f(g, \Omega)(T / J)^{-\nu(g, \Omega)}$.

A word of caution is necessary. We will examine mainly the temperature region $0.2 J \leq T \leq J$ but not extremely low temperatures. So the exponent $\nu(g, \Omega)$ we will determine in the following discussion describes the behavior of the correlation length in the examined temperature region only but not (necessarily) the behavior in the low temperature region. The exponent $\nu(g, \Omega)$ is not a critical exponent.

We first determine the best fit values of $\nu$ for the proportionality $\xi \propto T^{-\nu}$. Typical curves are shown in Fig. 4.31. Afterwards we examine the dependence $\nu=\nu(g, \Omega)$. The proportionality $1-\nu \propto 1 / \Omega$ is shown in Fig. 4.32a, the proportionality $1-\nu \propto g^{3 / 2}$ is 

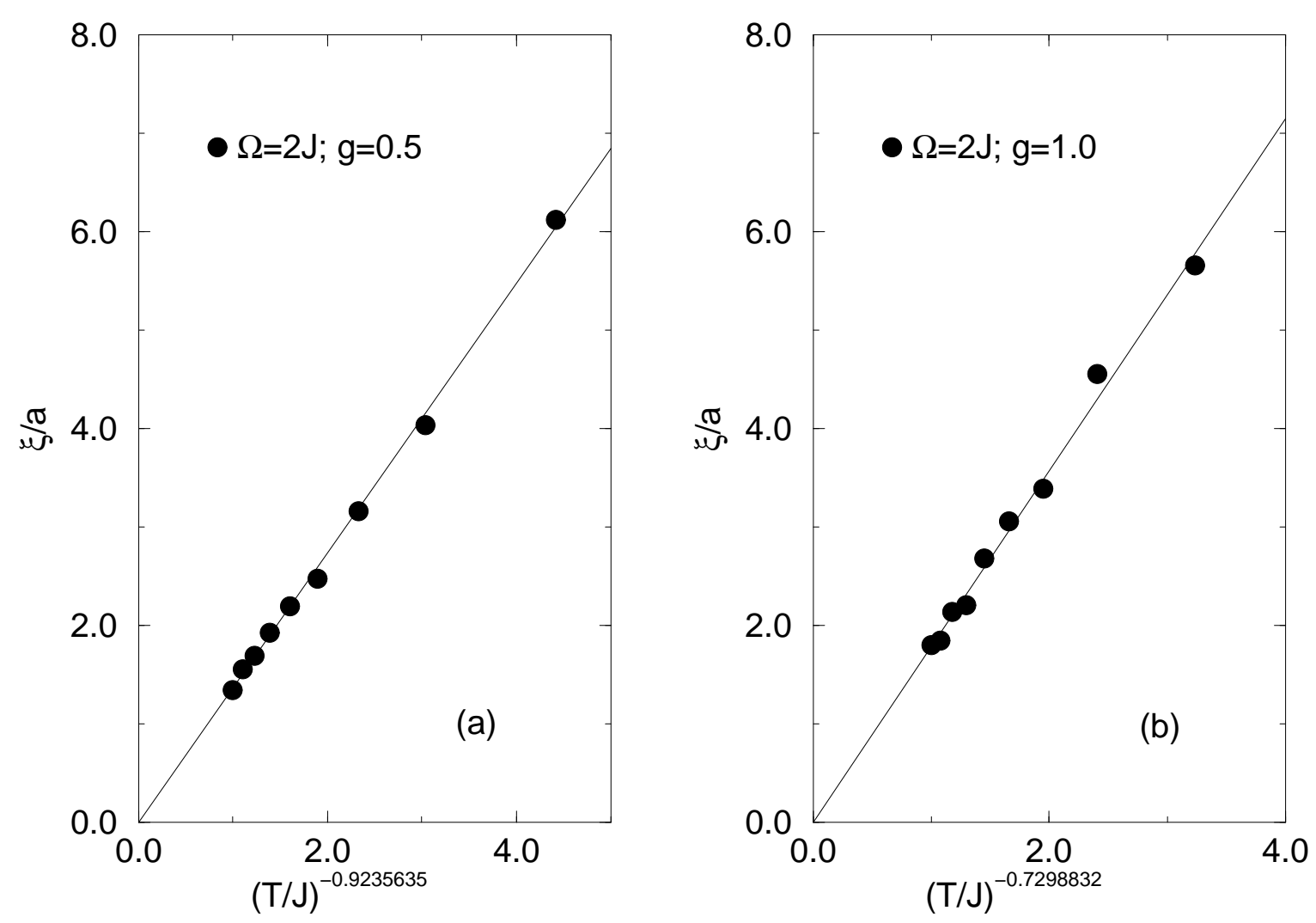

Figure 4.31: Correlation length $\xi$ in units of the lattice constant $a$ as a function of the best fit for the exponent $\nu$. The solid line is the linear fit. The error bar of $\nu$ can be estimated to be about 0.03 . Note that the fit for this figure is better than that for Fig. 4.30 where the same data were used.

shown in Fig. 4.32b. By using various sets for $g$ and $\Omega$ we find $1-\nu=0.5|g|^{3 / 2} J / \Omega$ (Fig. 4.33).

Naturally, this relation is valid only for systems with not too large values of $|g(\Omega)|$. For larger values of $g$ the dimerization becomes so strong that the correlation length approaches a finite value for $T \rightarrow 0$. This is why we were not able to identify exponents $\nu<0.7$ for high values of $|g|^{3 / 2} J / \Omega$.

According to the phase diagram of Raas [99] all our systems with $|g|^{3 / 2} J / \Omega \geq 0.3$ are in the dimerized phase. However, we investigate these systems only at temperatures above the gap temperature $T_{g}(g, \Omega)$. In this temperature region the effects of incomplete dimerization (step-like behavior of the staggered spin-spin correlation function) are clearly seen but we emphasize that this step-like behavior is only a small disturbance of the exponential decay of the spin-spin correlation function when large values of $|i-j|$ are 

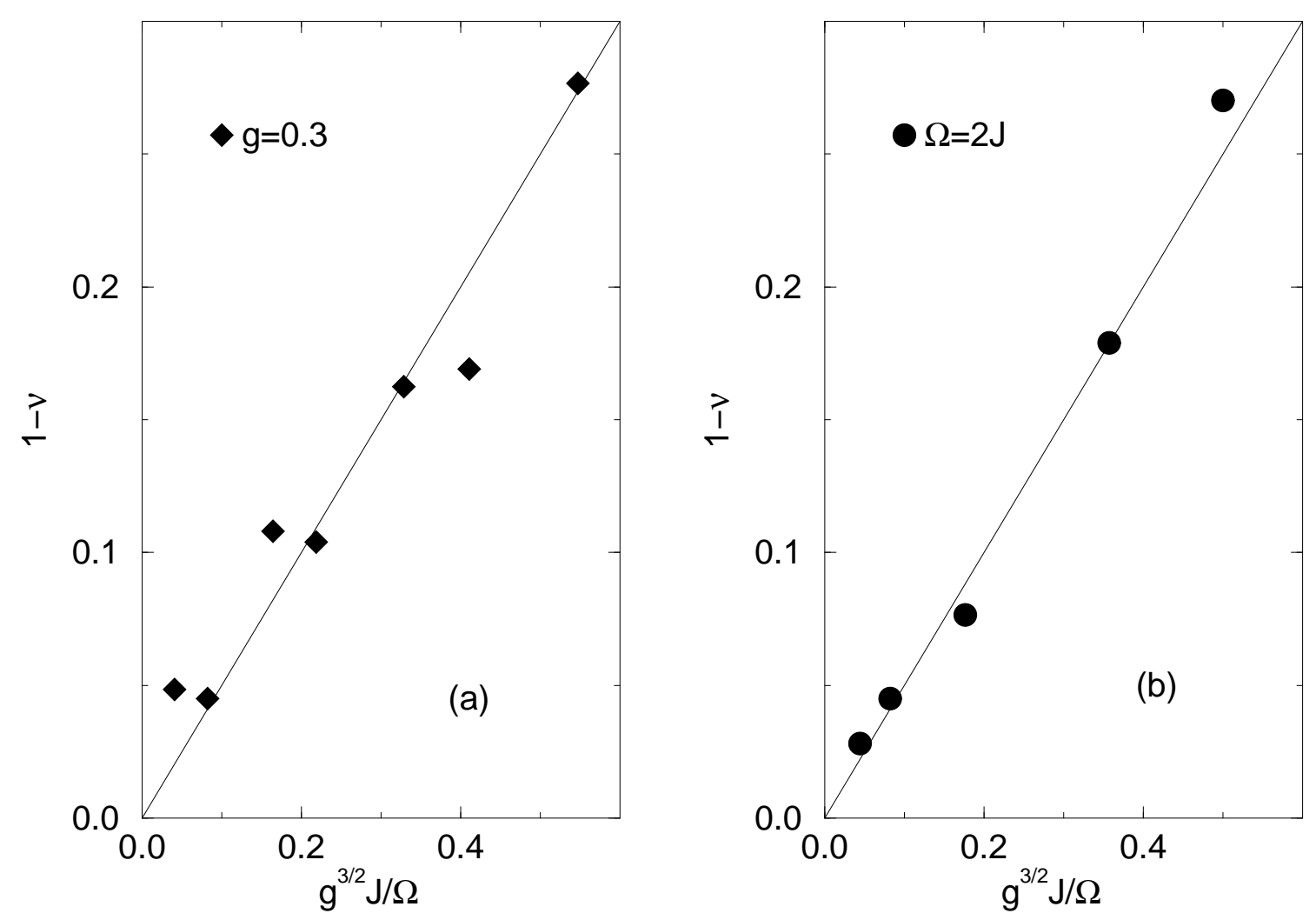

Figure 4.32: Deviation of the exponent $\nu$ from the Heisenberg value 1 in dependence on (a) $1 / \Omega$ and (b) $g^{3 / 2}$. The solid lines denote the relation $1-\nu=0.5 g^{3 / 2} J / \Omega$. The apparent step-like behavior of the left figure is most probably not real but an effect of the statistical fluctuations. The right figure clearly shows the proportionality $(1-\nu) \propto g^{3 / 2}$.

examined.

An important remark: When we tried to determine the phase diagram of the Heisenberg chain coupled to Einstein phonons, our quantum Monte Carlo approach required us to examine low but finite temperatures. A particular difficulty for the exact determination of the phase boundary was the smooth transition from the undimerized phase to the dimerized phase. The reason is that the systems of the dimerized phase are only incompletely dimerized and the systems of the undimerized phase show some incomplete dimerization resulting from the finite size of the chains investigated. Fortunately, it is exactly this smoothness at finite temperatures which allows us to investigate the exponent $\nu$ for some systems which are in the dimerized phase. The only condition is that these systems are not too far from the phase boundary. To give an example: For $\Omega=2 J$ the 


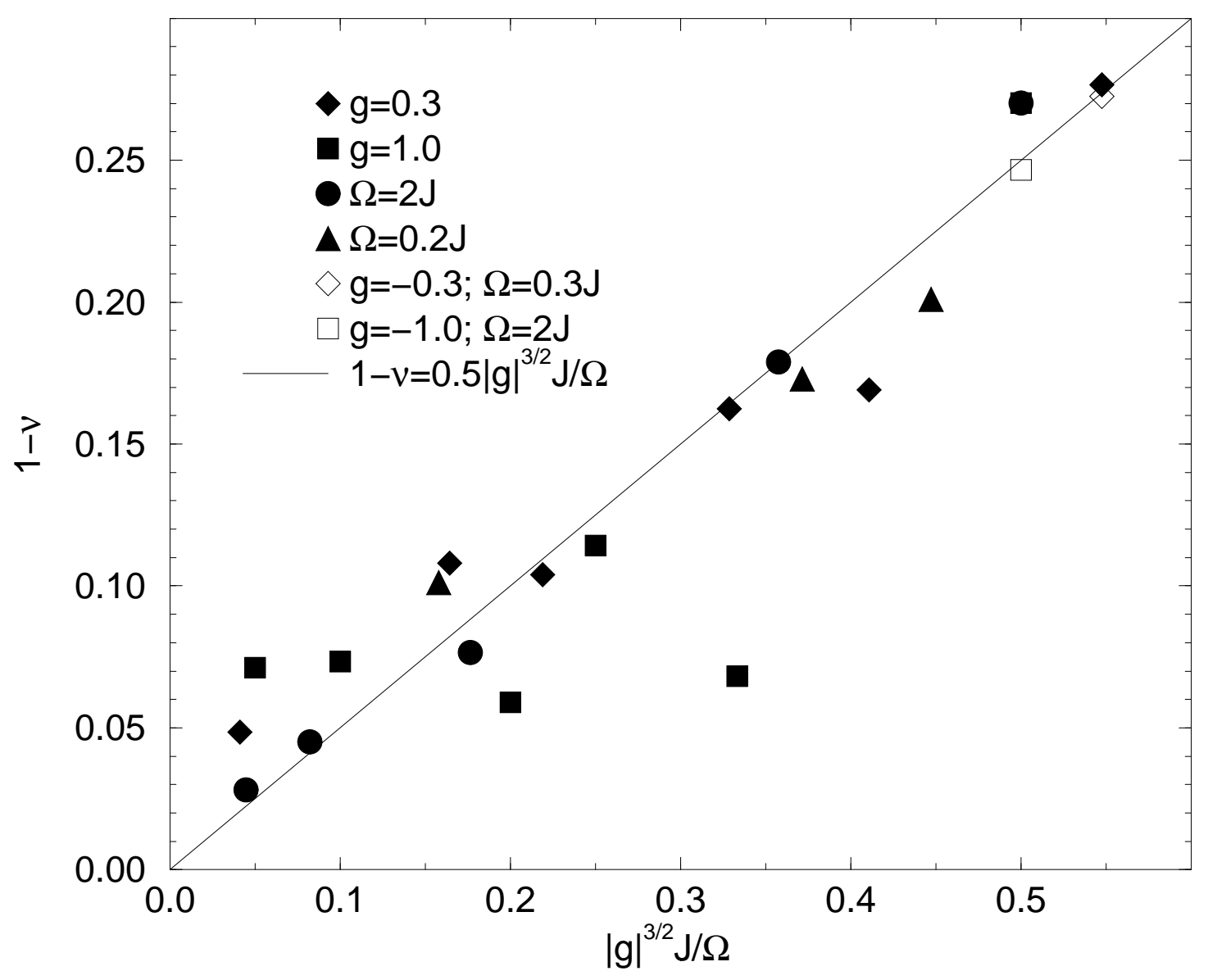

Figure 4.33: Deviation of the exponent $\nu$ from the Heisenberg value 1 as a function of $|g|^{3 / 2} J / \Omega$. For fixed $g=0.3$ and $g=1.0$ we varied the phonon frequency in the range $0.3 J \leq \Omega \leq 4 J$ and $2 J \leq \Omega \leq 20 J$, respectively. For fixed $\Omega=0.2 J$ and $\Omega=2 J$, respectively, we varied the spin-phonon coupling constant in the range $0.1 \leq g \leq 0.2$ and $0.2 \leq g \leq 1.0$, respectively.

phase boundary occurs at $g_{c}=0.59$ [99]. The exponent can be determined by our method both for the undimerized phase with $0 \leq g \leq g_{c}$ and for the dimerized phase provided that $g_{c} \leq g \leq 1.0$, where the staggered spin-spin correlation function decays exponentially, except from small perturbations of the step-like behavior which results from the incomplete dimerization. For larger spin-phonon couplings the step-like behavior destroys the exponential decay and the determination of $\nu$ becomes impossible.

Furthermore, it is important to point out that not all quantities are influenced by 

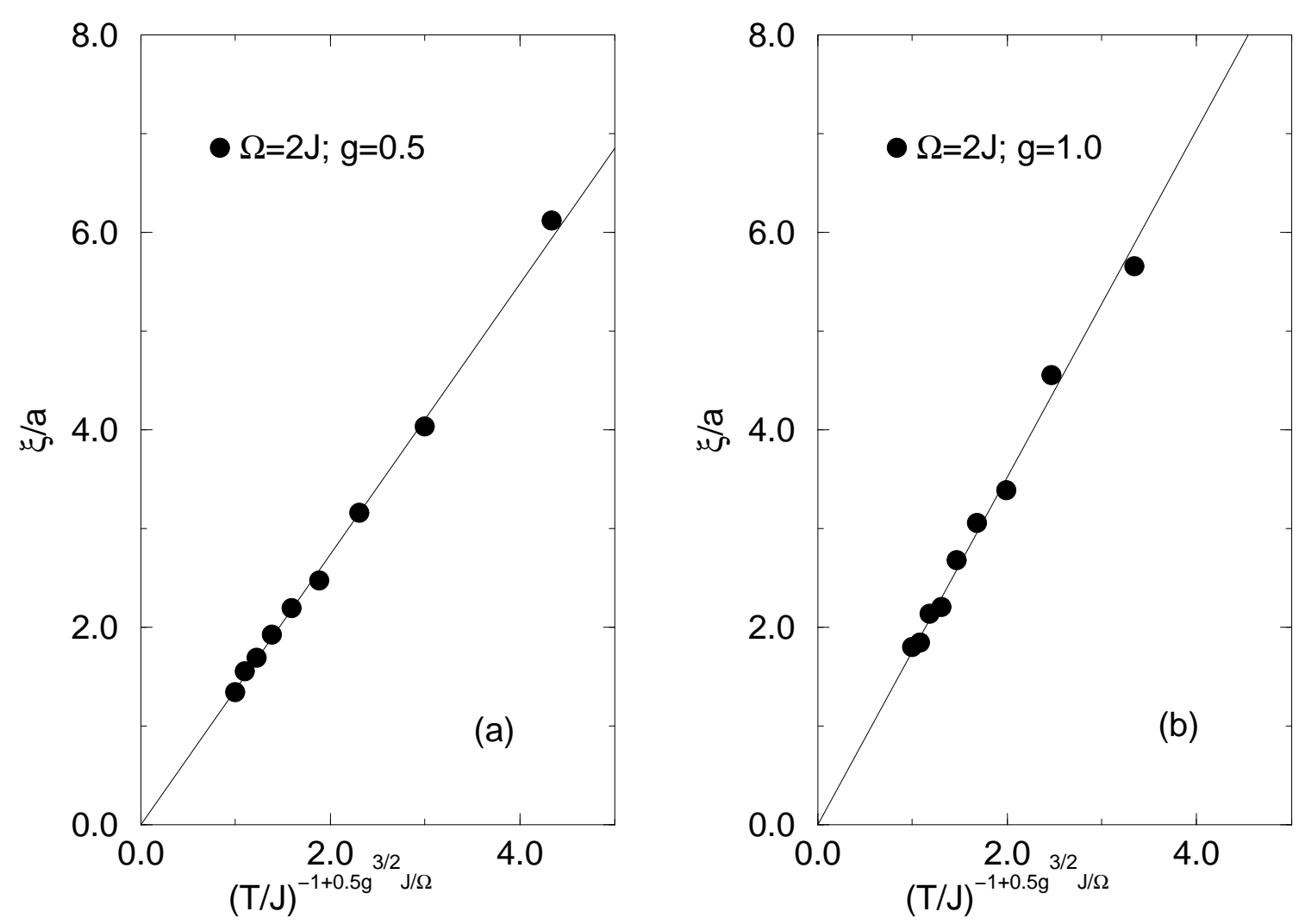

Figure 4.34: Correlation length $\xi$ in units of the lattice constant $a$ as a function of $(T / J)^{-\nu}$, where $\nu=1-0.5 g^{3 / 2} J / \Omega$. The solid line is the linear fit. The data are the same as for Figs. 4.30 and 4.31 .

the phase boundary. The mean phonon occupation number and the effective spin-spin coupling are examples. The independence of these quantities on whether the system is in the gapped or the gapless phase is the actual difficulty to determine the phase boundary via quantum Monte Carlo simulations at finite temperature. On the other hand, this independence makes it plausible why the expression for the exponent, $\nu=1-0.5|g|^{3 / 2} \mathrm{~J} / \Omega$, is valid both in the undimerized phase and in a small region of the dimerized phase near the phase boundary. Again, this expression was examined and verified for various sets of $g$ and $\Omega$ only in the temperature region $0.2 J \leq T \leq J$. We do not claim whether or not this expression is valid for even lower or higher temperatures.

We proceed by plotting $\xi$ as a function of $(T / J)^{-1+0.5|g|^{3 / 2} J / \Omega}$. Typical graphs are shown in Fig. 4.34. The slope parameter gives the value for $f(g, \Omega)$.

The dependence of $f$ on $\Omega$ is shown in Figs. 4.35a and c, that on $g$ in Figs. 4.35b and 

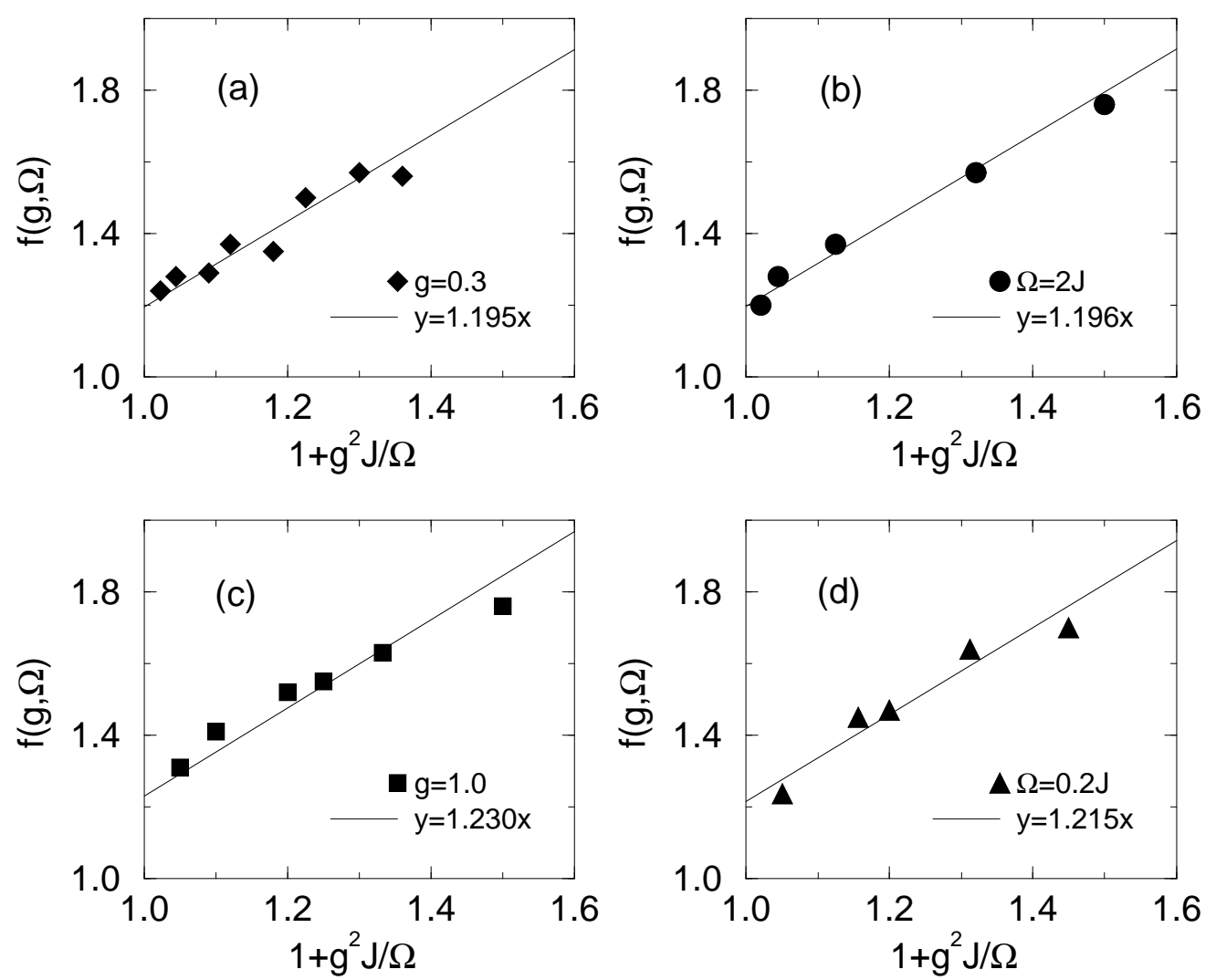

Figure 4.35: The factor $f(g, \Omega)$ as (a) a function of $\Omega,(\mathrm{b})$ a function of $g$, (c) a function of $\Omega$, and (d) a function of $g$. The solid lines are linear fits. Ideally, they should be identical.

d. We find $f(g, \Omega)=c\left(1+g^{2} J / \Omega\right)$. The proportionality constant is $c=1.215 \pm 0.020$ (Fig. 4.36).

More precisely, this "proportionality constant" should be identical with the temperature dependent function $c_{H}(T)$ of the Heisenberg chain. In Fig. 4.37 we show the correlation length versus temperature for a system with $g=1.0, \Omega=2 \mathrm{~J}$, and $0.025 \mathrm{~J} \leq$ $T \leq J$. The data of the magnetic susceptibility yield the spin gap of this system to be $\Delta=(0.065 \pm 0.005) J$. Quite remarkably, the identity is satisfied not only for temperatures above the spin gap, but also within the gap, at least until temperatures $T=0.025 \mathrm{~J}$. For lower temperatures we expect that this identity is no longer satisfied and that $\xi$ will tend towards a finite value for $T \rightarrow 0$.

Furthermore, we plot $\xi$ rescaled with $1 /\left(1+g^{2} J / \Omega\right)$ as a function of $(T / J)^{-1+0.5|g|^{3 / 2} J / \Omega}$, see Fig. 4.38. The slope parameter gives the value for $c(T)$. We have to point out that this figure shows the behavior of the systems only for the temperature region $0.2 J \leq T \leq J$. 


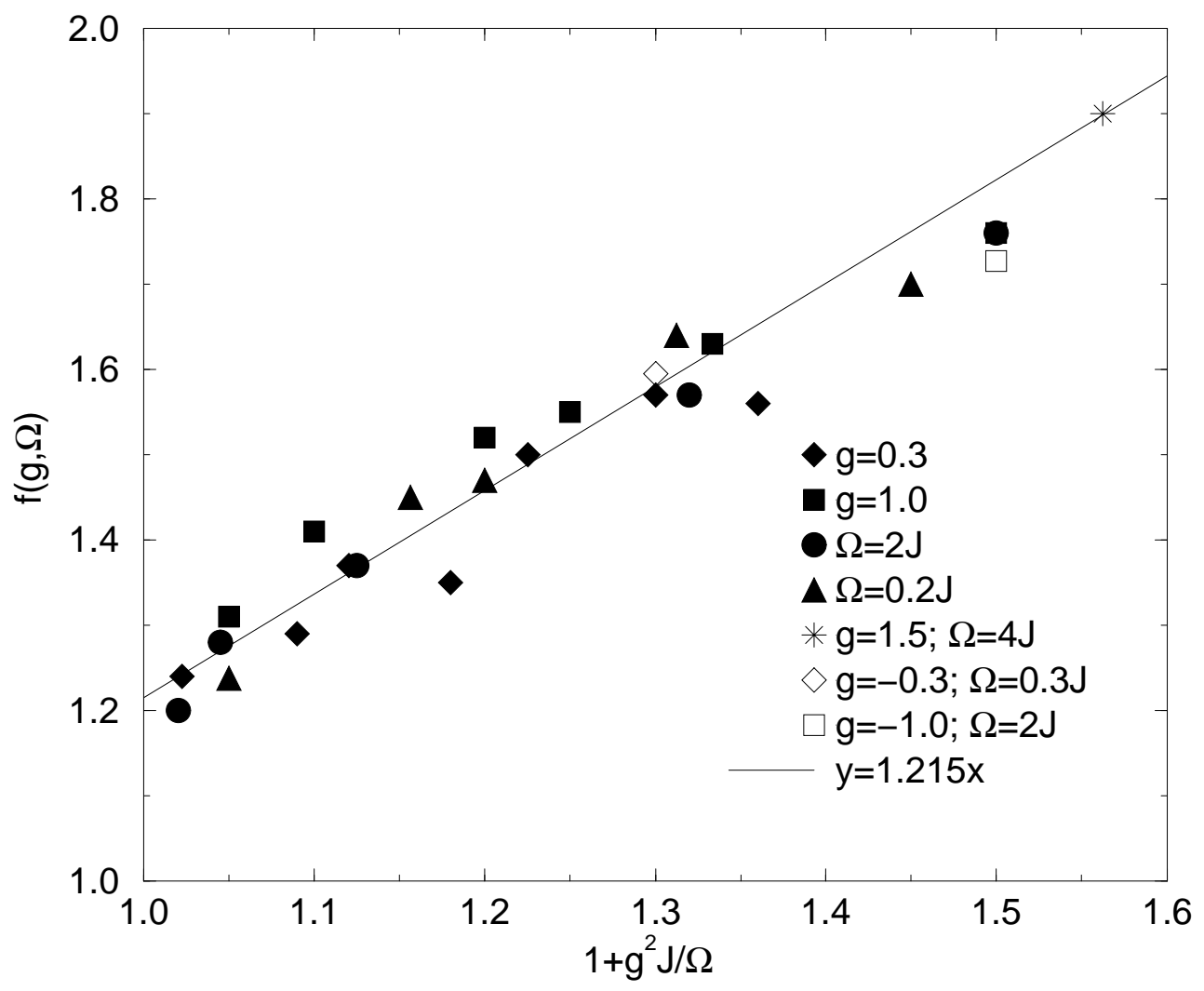

Figure 4.36: The factor $f(g, \Omega)$ as a function of both $g$ and $\Omega$. The solid line is the linear fit.

For lower temperatures we expect deviations from the obvious linear dependence shown in the figure. Indeed, the strongly dimerized system with $\Omega=0.2 J$ and $g=0.3$ whose data are included in Fig. 4.38 already shows a deviation from the dependence. Its linear behavior is satisfied only for $(\xi / a) /\left(1+g^{2} J / \Omega\right) \leq 2.0$.

To test our Eq. (4.26) we present $\xi$ for fixed temperature $T=J$ and various choices of $g$ and $\Omega$ in Figs. 4.39, 4.40. For $T=J$ this equation simplifies to $\xi / a=f(g, \Omega) \equiv$ $c\left(1+g^{2} J / \Omega\right)$. We confirm Eq. (4.26) where our best fit yields $c=1.212$. This is in agreement with the exact result for the Heisenberg chain, $c_{H}(T=J)=1.203$ (Ref. [19]).

The correlation lengths of all our runs can be fitted by the proportionality $\xi \propto(T / J)^{-\nu}$ where $\nu=1-0.5|g|^{3 / 2} J / \Omega$ (typical graphs are shown in Fig. 4.34). Furthermore, for $T=J$ the correlation lengths of all our runs satisfy $\xi / a=c\left(1+g^{2} J / \Omega\right)$ where the proportionality constant $c$ is in very good agreement with the value for the Heisenberg chain (Fig. 4.40). The variation of $\nu$ with $g$ and $\Omega$ is real and not a spurious artifact from a non-power-law behavior of the correlation length. Moreover there is no significant dependence on the 


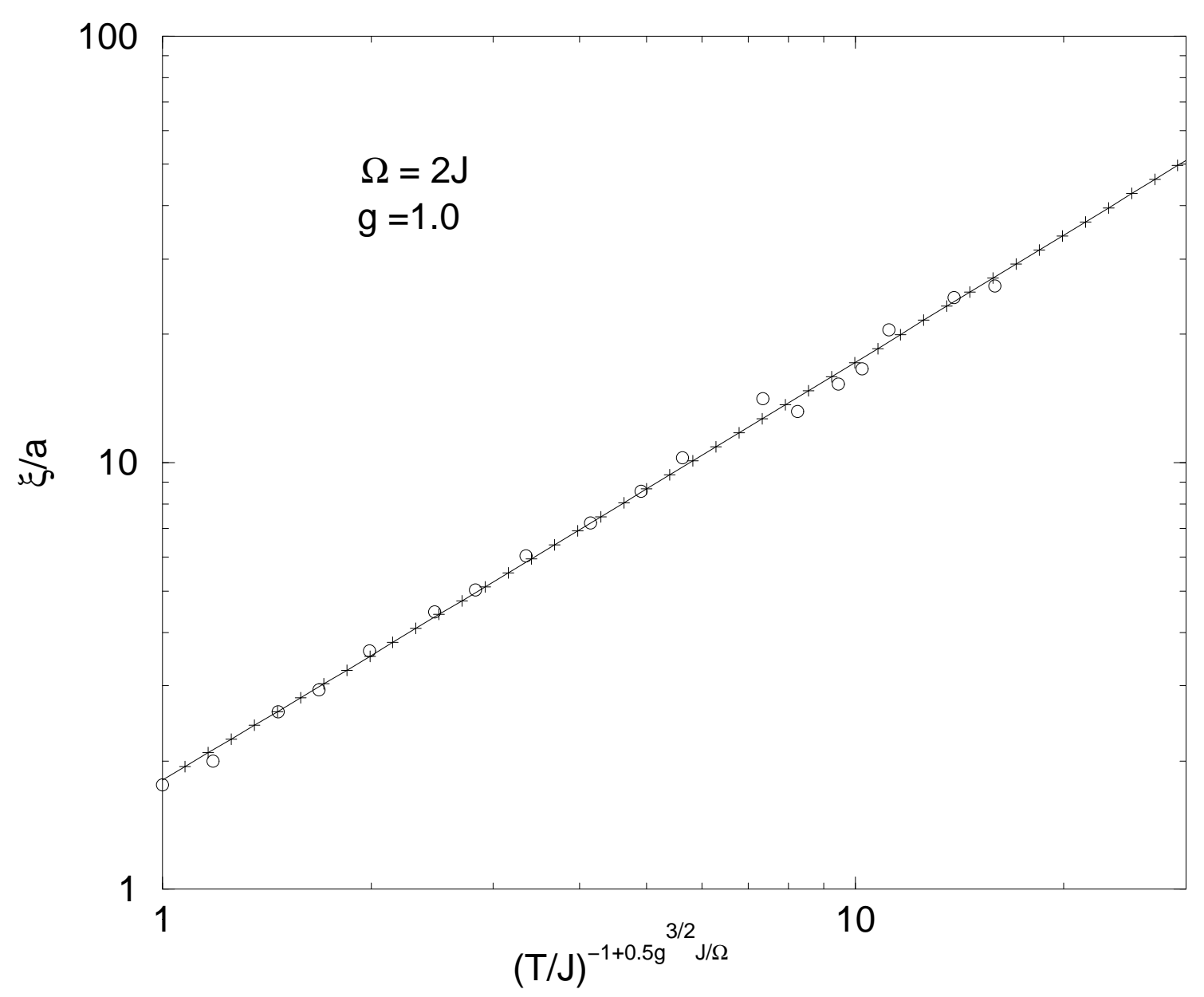

Figure 4.37: Correlation length versus temperature plotted double-logarithmically for $\Omega=2 J$ and $g=1.0$. Chain length and Trotter number are $N=64$ and $M=80$ for $0.2 \leq T / J \leq 1.0$ (lower 8 circles) and $N=256$ and $M=320$ for $0.025 \leq T / J \leq 0.15$ (upper 10 circles). The curve marked with crosses denotes the expected function $\xi / a=$ $c_{H}(T)\left(1+g^{2} J / \Omega\right)(T / J)^{-1+g^{3 / 2} J /(2 \Omega)}$, where the crosses denote the rescaled values for the Heisenberg chain $c_{H}(T)$ from Ref. [19].

finite Trotter number in the investigated temperature region. The possible dependence of the correlation length on the finite Trotter number is shown in Figs. 4.41-4.44. The correlation lengths are plotted versus inverse temperature (Figs. 4.41 and 4.42) and versus $(T / J)^{-1+0.5|g|^{3 / 2} J / \Omega}$ (Figs. 4.43 and 4.44 ), respectively.

The data presented in Fig. 4.37 are particularly remarkable, because they include various tests of our procedure.

(i) The high temperature data $(T \geq 0.2 J)$ were obtained with short chain length $N=64$ and small Trotter number $M=80$. Those at low temperatures $(T \leq 0.15 J)$ with large chain length $N=256$ and large Trotter number $M=320$. No systematical effects 


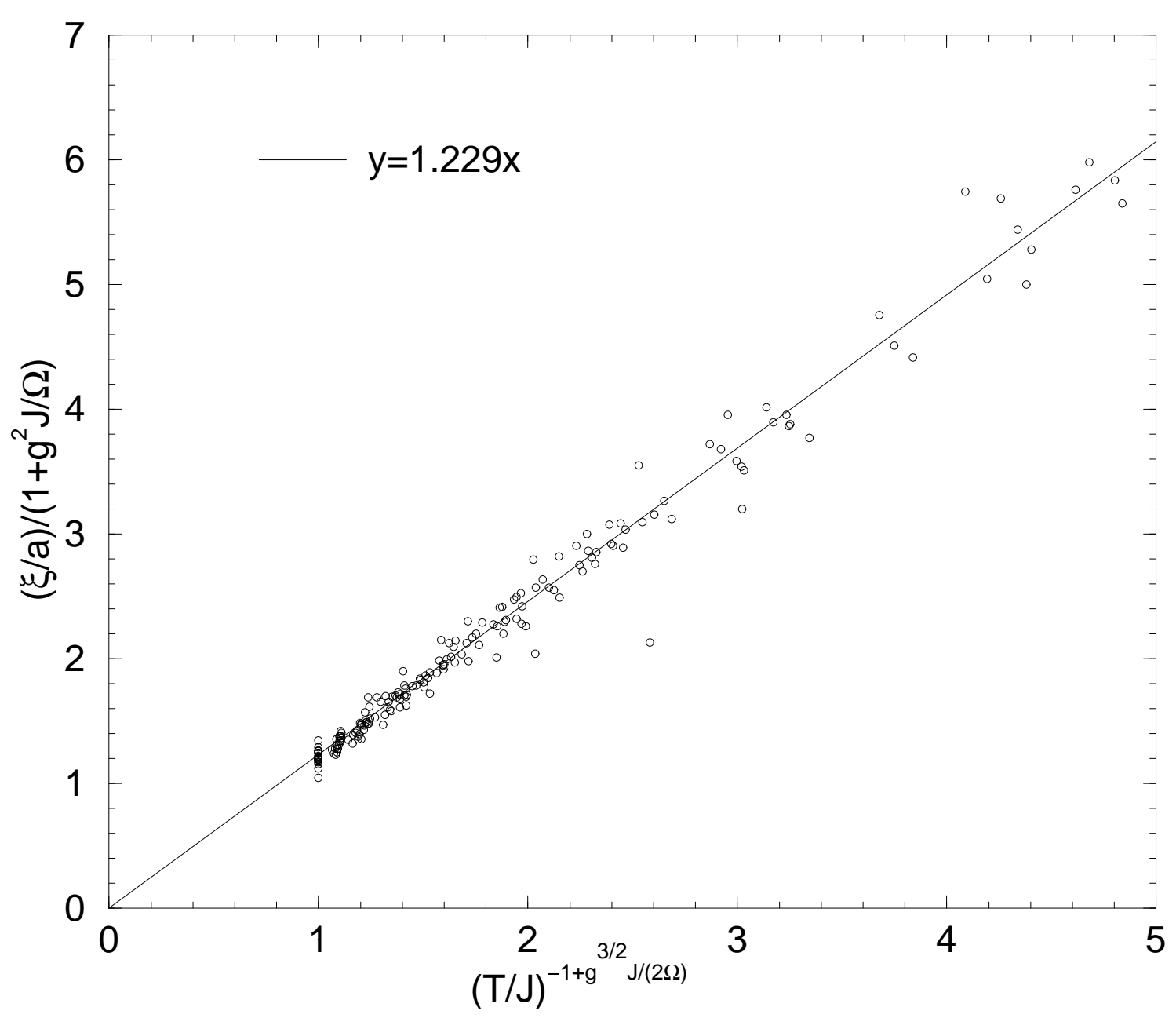

Figure 4.38: Correlation length $\xi$ in units of the lattice constant $a$ rescaled with $1 /(1+$ $\left.g^{2} J / \Omega\right)$ as a function of $(T / J)^{-\nu}$, where $\nu=1-0.5 g^{3 / 2} J / \Omega$. Presented are the correlation lenghts for all systems at all temperatures we investigated, 192 data points in total. The solid line is the linear fit.

(e. g. a step) are detectable in the plot at temperature $T=0.2 J$. This rules out the possibility of large systematical errors due to finite size and finite Trotter number.

(ii) The data points are scattered symmetrically around the expected curve, Eq. (4.26). Hence, systematical errors are negligible compared to the statistical errors of $5 \%$. This error is small enough to allow the determination of the exponent with high precision. This is because the correlation length increases from $\xi=1.76 a$ at $T=J$ by a factor of $\sim 15$ to $\xi=25.97 a$ at $T=0.025 \mathrm{~J}$. The best fit for the exponent is $\nu=0.74$. The data cannot be fitted with the value $\nu=1$ of the Heisenberg chain.

(iii) The absence of any detectable systematical deviation shows clearly that errors resulting from the finite thermalization time are not significant. 

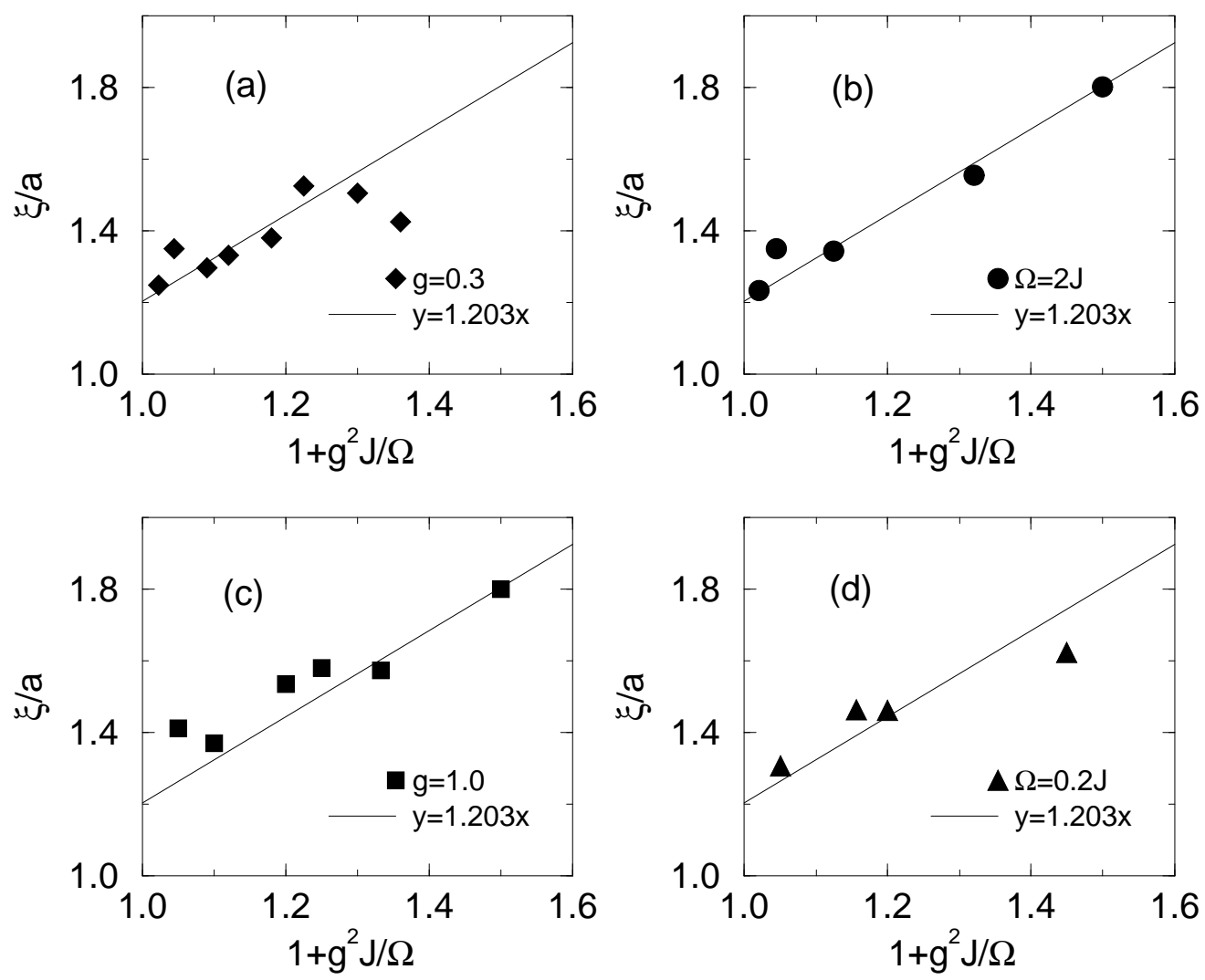

Figure 4.39: Correlation length $\xi / a$ as a function of $1+g^{2} J / \Omega$ for fixed temperature $T=J$. $[(a, c)]: g$ is fixed, $\Omega$ is varied; $[(b, d)]: \Omega$ is fixed, $g$ is varied. The slope of the solid lines is the value $c_{H}=1.203$ of the Heisenberg model.

The dependence of the exponent on a non-integral power of the coupling constant appears somewhat unconventional. One might suppose that this proportionality could be explained as a simple fit to a more natural integral power dependence. To test this possibility we tried to replace $1-\nu=0.5|g|^{3 / 2} J / \Omega$ by several functions. For example we tried the test function $1-\nu=\alpha|g| J / \Omega+\beta(g J / \Omega)^{2}$, where we tried to determine best fits for the constant free parameters $\alpha$ and $\beta$. However, the fits were always worse than that for the original dependence $1-\nu=0.5|g|^{3 / 2} \mathrm{~J} / \Omega$, which has only one parameter that was determined to be 0.5 . We conclude that the unconventional non-integral power dependence on the spin-phonon coupling constant cannot be explained as an artificial fit to integral power dependencies.

Furthermore, we investigate a system with $\Omega=J$ and $g=1.0$. According to the phase diagram, Fig. 4.20, this system is in the gapped phase and far away from the phase 


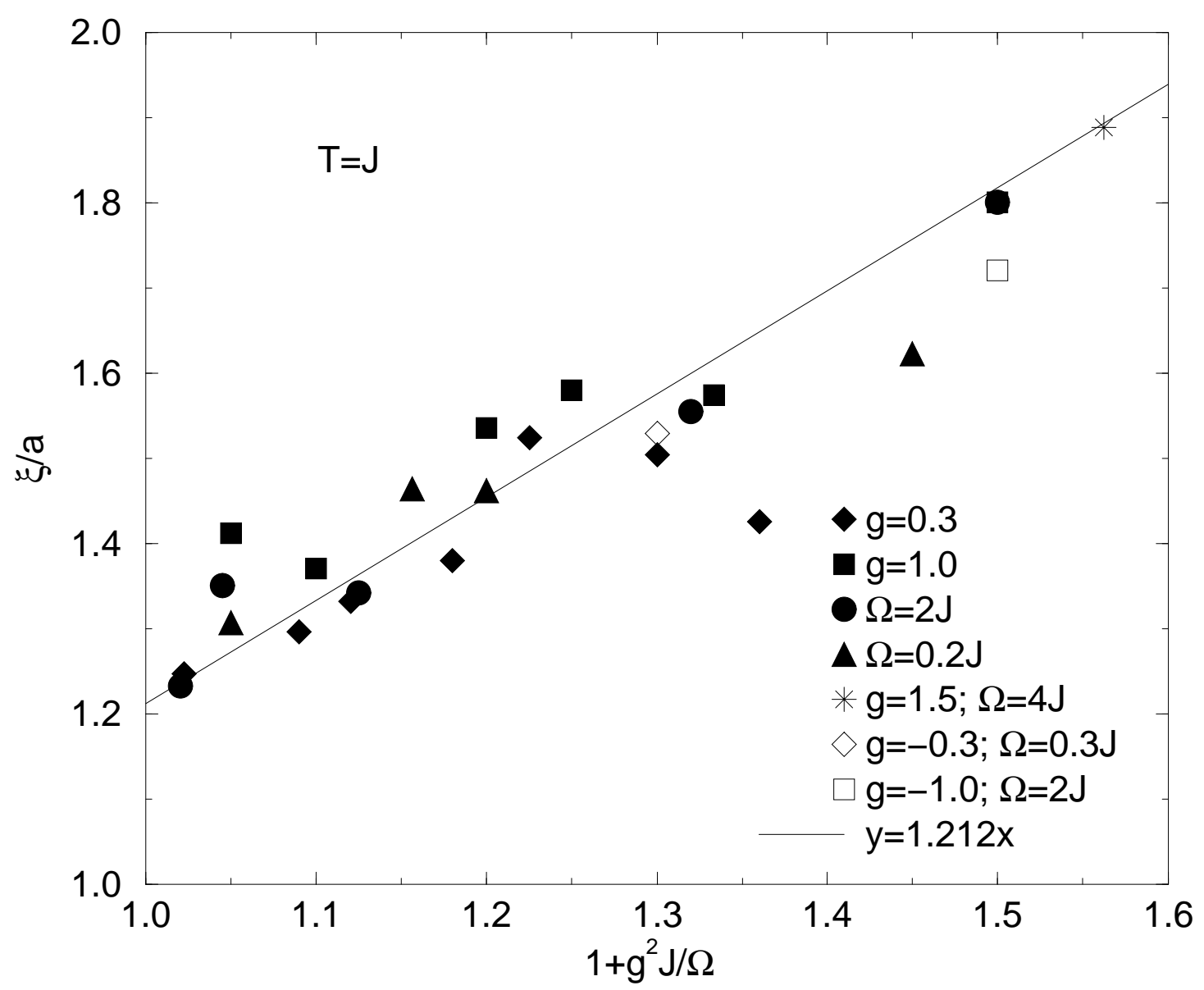

Figure 4.40: Correlation length $\xi / a$ as a function of $1+g^{2} J / \Omega$ for fixed temperature $T=J$. The data of Fig. 4.39 are presented in one diagram. The solid line is the linear fit.

boundary. Our data for the magnetic susceptibility yield the gap of this system to be $\Delta=(0.37 \pm 0.02) J$. Slightly above the gap temperature (at $T \simeq 0.5 J)$ the correlation length has a maximum. Within the gap a minimum appears at $T \simeq 0.2 \mathrm{~J}$, see Fig. 4.45 . More work is needed to understand why the phononic degrees of freedom appear to show this anomalous effect.

In summary, in the temperature range $0.2 J \leq T \leq J$ we found evidence for a powerlaw behavior of the temperature dependence of the spin-spin correlation length of the spin-1/2 isotropic antiferromagnetic Heisenberg chain coupled to Einstein phonons. The dependence of the correlation length $\xi$ on the phonon frequency $\Omega$ and the spin-phonon 


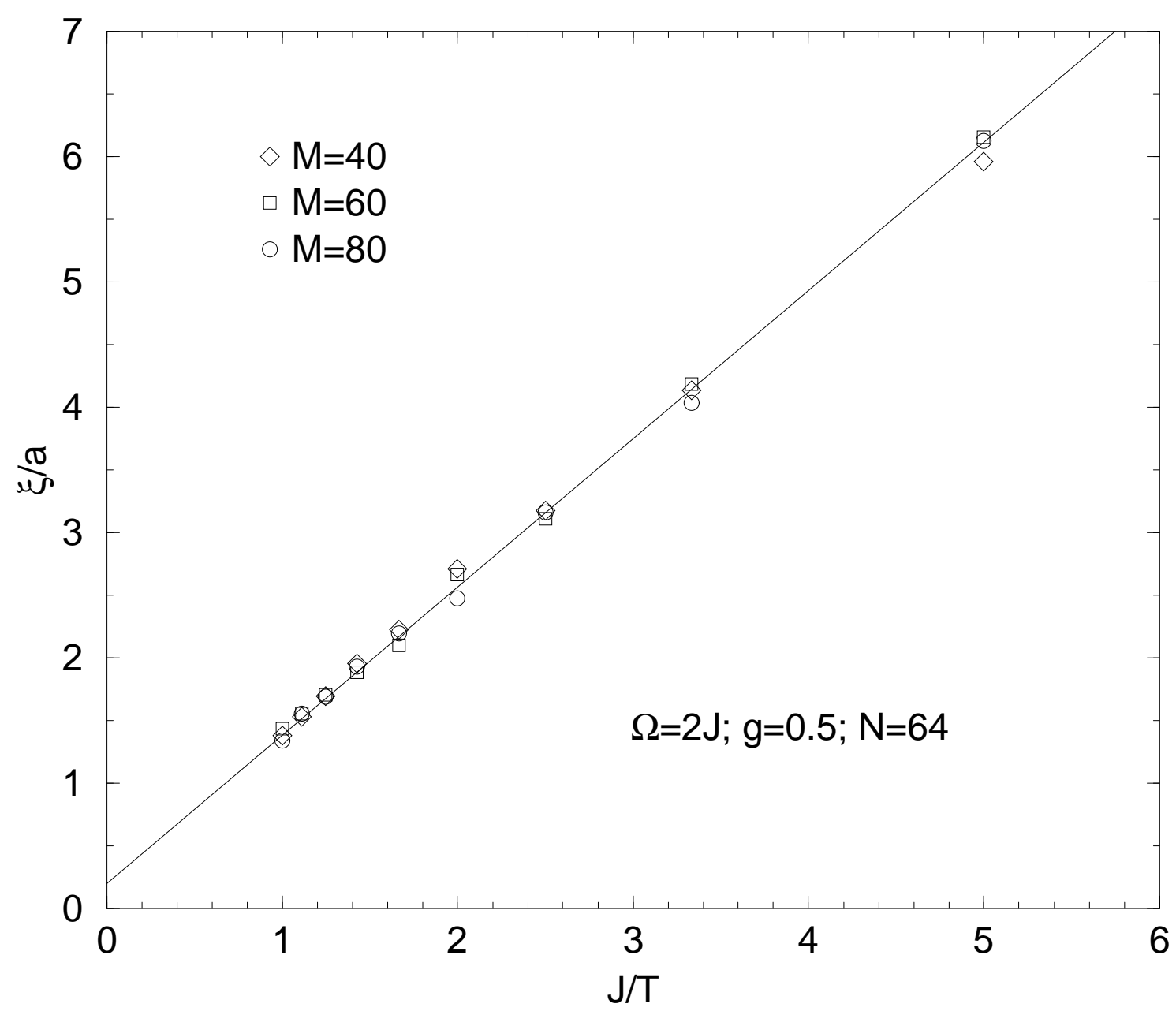

Figure 4.41: Correlation length $\xi$ versus the inverse temperature. The Trotter number $M$ is varied between 40 and 80 . Statistical fluctuations clearly dominate systematical deviations from the finite Trotter number. The solid line shows the linear fit.

coupling constant $g$ is

$$
\frac{\xi}{a}=(1.215 \pm 0.020)\left(1+\frac{g^{2} J}{\Omega}\right)\left(\frac{T}{J}\right)^{-1+0.5|g|^{3 / 2} J / \Omega}
$$

where $a$ is the lattice constant, $T$ is the temperature, and $J$ is the spin-spin coupling constant. The value $c=1.215 \pm 0.020$ is in accordance with the value found for the Heisenberg chain, $c_{H}=1.18 \pm 0.03$, in the temperature range $0.2 J \leq T \leq J$ (Ref. [19]). In this temperature range $c_{H}$ varies slightly (by $5 \%$; possibly due to logarithmic corrections). From our data sets (cf. Fig. 4.34) we can infer an upper limit for the variation of $c$ in this temperature range to be $10 \%$.

This result may be of importance for the understanding of materials like $\mathrm{Sr}_{2} \mathrm{CuO}_{3}$ and 


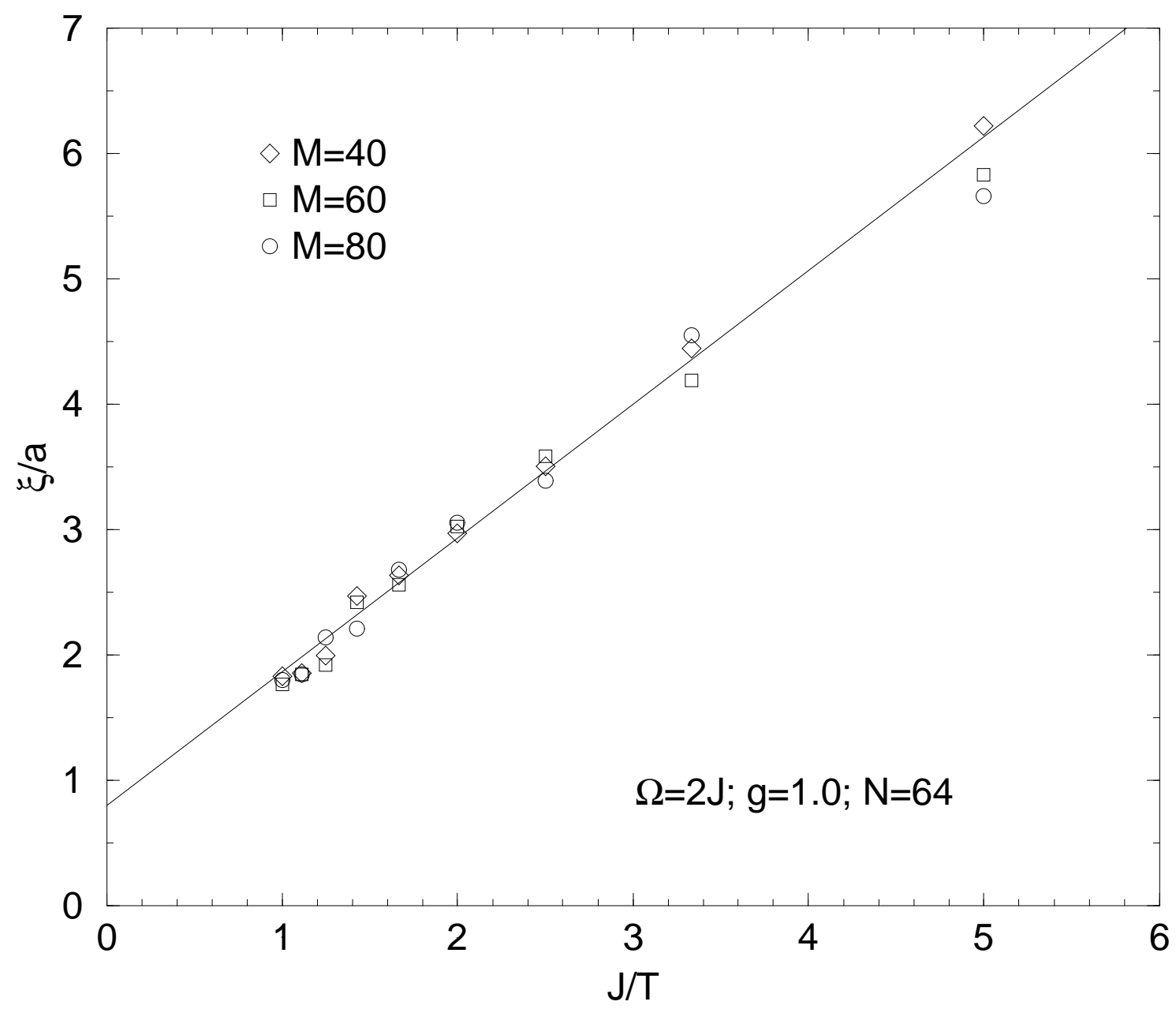

Figure 4.42: Similar to Fig. 4.41 we plot the correlation length $\xi$ versus the inverse temperature. Here, however, we show data for the larger spin-phonon coupling $g=1.0$ instead of $g=0.5$. The Trotter number $M$ is varied between 40 and 80 . Statistical fluctuations clearly dominate systematical deviations from the finite Trotter number (at least for temperatures $T \geq 0.3 J$ ). The solid line shows the linear fit.

$\alpha^{\prime}-\mathrm{NaV}_{2} \mathrm{O}_{5}$ whose (high-temperature) dynamics can be described by a nearest-neighbor spin-1/2 isotropic antiferromagnetic Heisenberg chain $[5,53]$.

A word of caution is required. Strictly, we have shown only that Eq. (4.26) is a good fit for the correlation lengths in the temperature range $0.2 J \leq T \leq J$ for Heisenberg chains coupled to Einstein phonons which are either in the gapless phase or in a small region of the gapped phase near the phase boundary. We emphasize that the formula is most likely not valid in those regions of the gapped phase which are far from the phase boundary. Moreover we have not proven that the formula is valid for very low temperatures.

One can expect that at very low temperatures the correlation length does not satisfy 


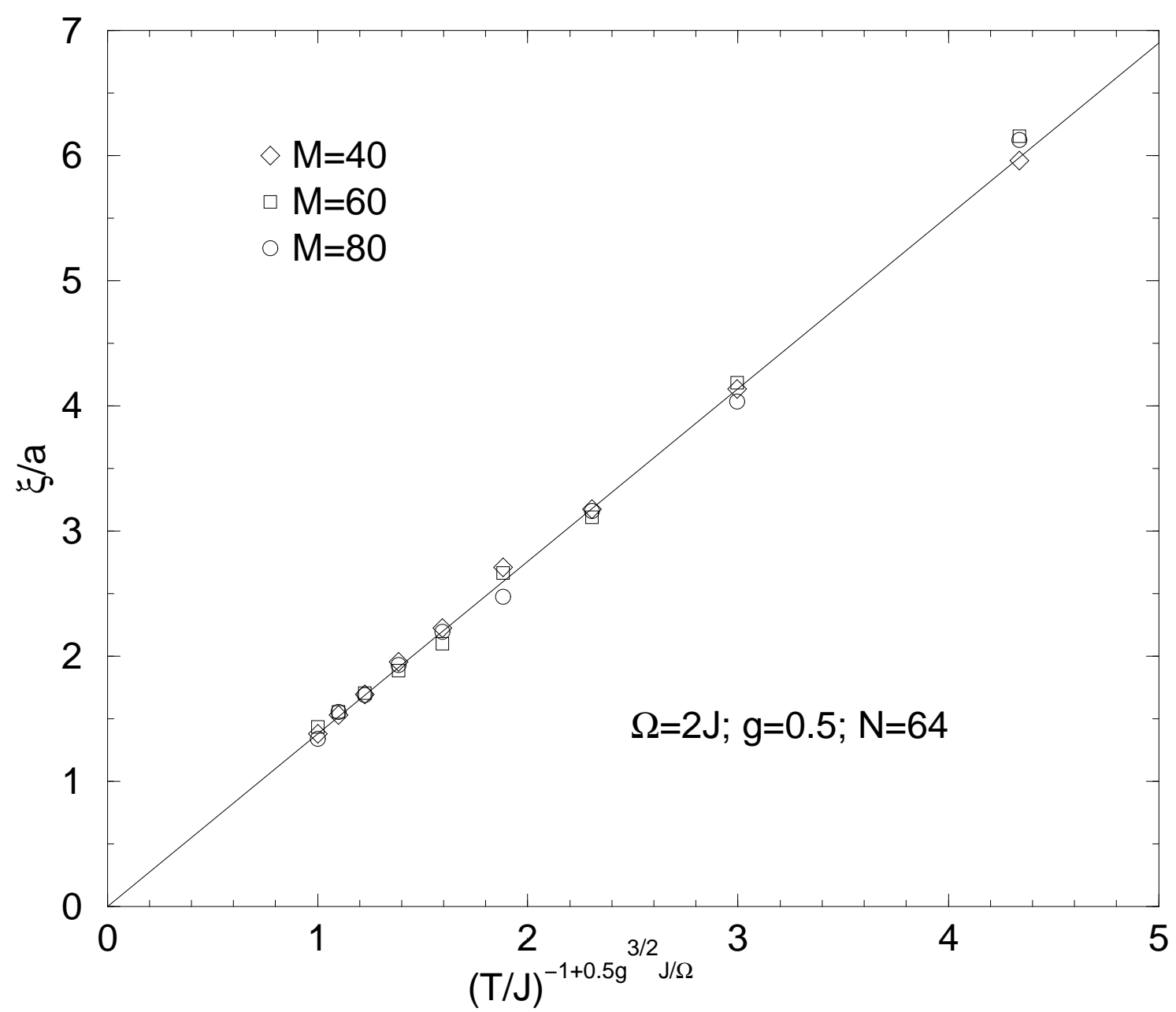

Figure 4.43: Correlation length $\xi$ versus $(T / J)^{-\nu}$, where $\nu=1-0.5 g^{3 / 2} J / \Omega$. The Trotter number $M$ is varied between 40 and 80 . Statistical fluctuations clearly dominate systematical deviations from the finite Trotter number. The solid line shows the linear fit.

Eq. (4.26). In the gapless phase one would expect the critical exponent $\nu=1$ and in the gapped phase one would expect the behavior

$$
\xi / a=a_{1}-a_{2} T^{2}+O\left(T^{3}\right) \quad \text { for } \quad T \rightarrow 0,
$$

where $a_{1}$ and $a_{2}$ are positive constants [114]. 


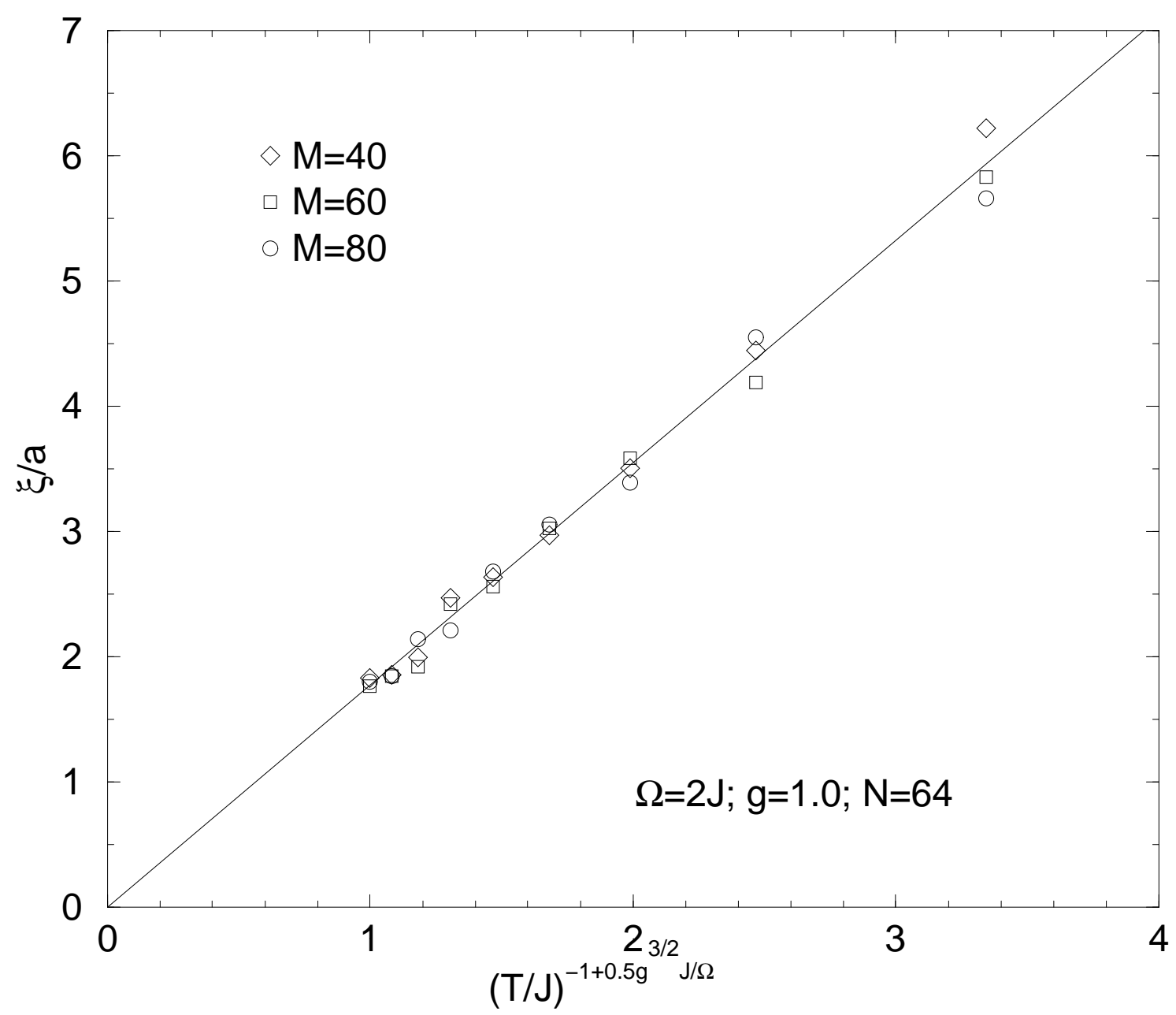

Figure 4.44: Similar to Fig. 4.43 we plot the correlation length $\xi$ versus $(T / J)^{-\nu}$, where $\nu=1-0.5 g^{3 / 2} J / \Omega$. Here, however, we show data for the larger spin-phonon coupling $g=1.0$ instead of $g=0.5$. The Trotter number $M$ is varied between 40 and 80 . Statistical fluctuations clearly dominate systematical deviations from the finite Trotter number (at least for temperatures $T \geq 0.3 J)$. The solid line shows the linear fit. 


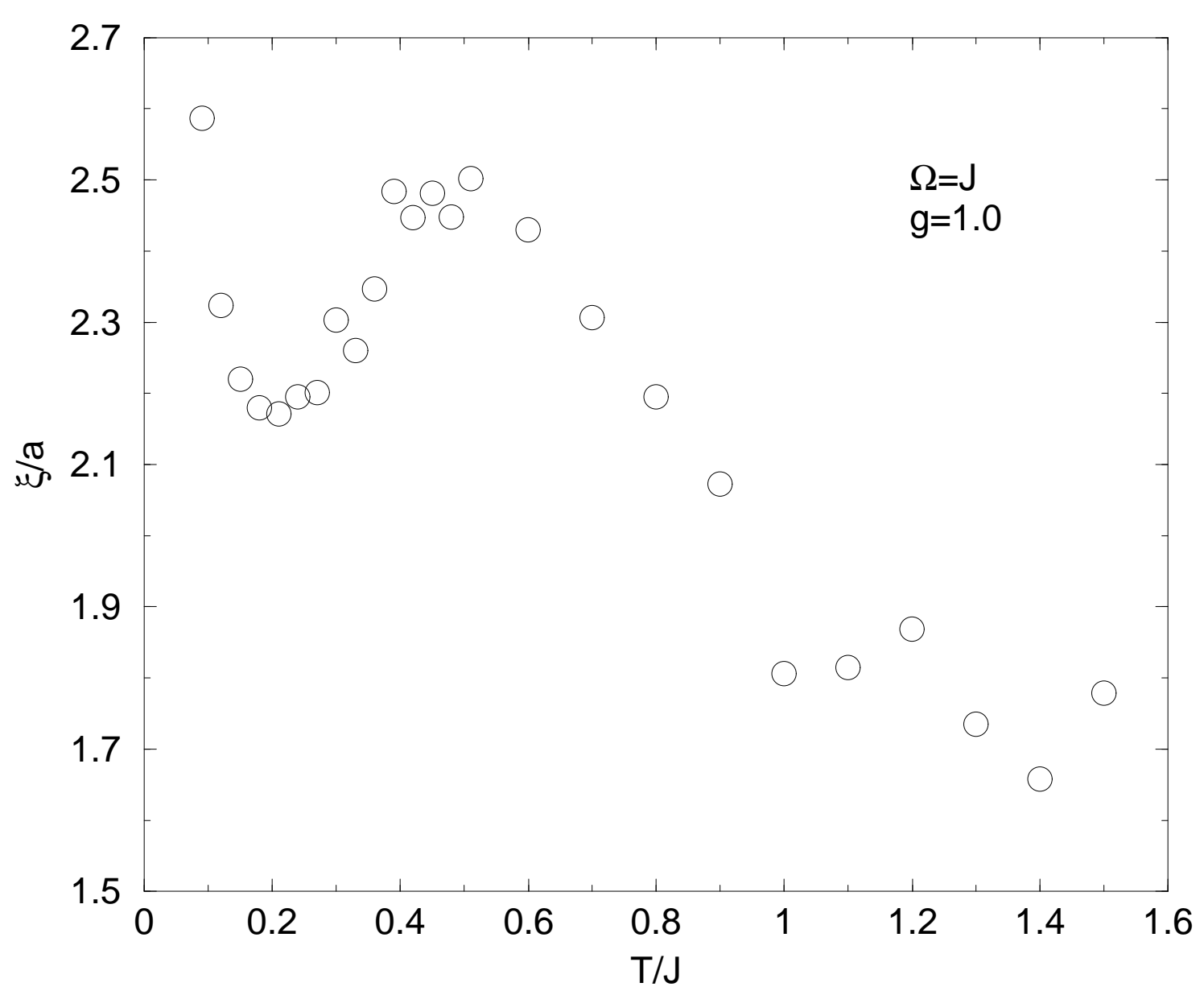

Figure 4.45: Correlation length versus temperature for the strongly dimerized chain with $\Omega=J$ and $g=1.0$. The chain length and Trotter number are $N=64$ and $M=80$. Remarkable features are the minimum at $T=0.2 \mathrm{~J}$ and the maximum at $T=0.5 \mathrm{~J}$. The gap for this system is $\Delta=(0.37 \pm 0.02) \mathrm{J}$. 


\section{Chapter 5}

\section{Two-frequency model}

The spin-Peierls phase-transition of $\mathrm{CuGeO}_{3}$ is generated mainly by phonons of two different frequencies. This prompted us to discuss not only systems with a single frequency, but to examine also models with two frequencies $\Omega_{1}$ and $\Omega_{2}$. To do this we had to modify our quantum Monte Carlo algorithm by doubling the number of Trotter layers. This becomes posssible, because the ladder operators of the two phonon species commute. The Hamiltonian of this system is,

$$
\begin{gathered}
\mathcal{H}=\frac{J}{2} \sum_{l=1}^{N}\left(\vec{\sigma}_{l} \vec{\sigma}_{l+1}-1\right)\left(1+g_{1}\left(a_{l}^{\dagger}+a_{l}\right)+g_{2}\left(b_{l}^{\dagger}+b_{l}\right)\right) \\
+\Omega_{1} \sum_{l=1}^{N}\left(a_{l}^{\dagger} a_{l}+\frac{1}{2}\right)+\Omega_{2} \sum_{l=1}^{N}\left(b_{l}^{\dagger} b_{l}+\frac{1}{2}\right) .
\end{gathered}
$$

The values of the spin-spin coupling constant, the frequencies and spin-phonon coupling constants of the spin-Peierls phase-transition generating phonons of $\mathrm{CuGeO}_{3}$ have been determined experimentally. However, their values are not yet precisely known. Braden et al. measured $J=1.7 \mathrm{THz}[92], \Omega_{1}=3.2 \mathrm{THz}$ and $\Omega_{2}=6.8 \mathrm{THz}$ [51]. Gros and Werner [93] found $\Omega_{1}=3.13 \mathrm{THz}=151 \mathrm{~K}, \Omega_{2}=6.65 \mathrm{THz}=317 \mathrm{~K}, g_{1} /(2 J)=0.45 \mathrm{THz}$, and $g_{2} \simeq 4 g_{1}$. In a later publication, Werner et al. [52] found $\Omega_{1}=3.12 \mathrm{THz}, \Omega_{2}=6.53 \mathrm{THz}$, $g_{1} /(2 J)=-15 \mathrm{~K}$, and $g_{2} /(2 J)=58 \mathrm{~K}$. To conclude, we have approximately $\Omega_{1} \simeq 2 J$, $\Omega_{2} \simeq 4 J, g_{1} \simeq-3 / 8$, and $g_{2} \simeq 3 / 2$.

The frequencies and the spin-phonon coupling constants of the spin-Peierls phasetransition generating phonons of $\mathrm{NaV}_{2} \mathrm{O}_{5}$ have not yet been determined.

The magnetic susceptibility for various systems with three values of the spin-phonon coupling constants is shown in Fig. 5.1. The parameters of one system are those of $\mathrm{CuGeO}_{3}$, but with the frustration neglected. The precise value of the frustration parameter is under discussion. Recent studies yielded $\alpha \simeq 0.24$ [46], $\alpha \simeq 0.354$ [48], and $\alpha \simeq 0.36$ [47] for $\mathrm{CuGeO}_{3}$. The dependence of the finite chain length is shown in Fig. 5.2. When comparing these data with those of the one-frequency models, we find that neither the statistical fluctuations nor the finite-size effects increase with the addition of the second frequency. Hence, two-frequency models can be examined as easily as the one-frequency models. 


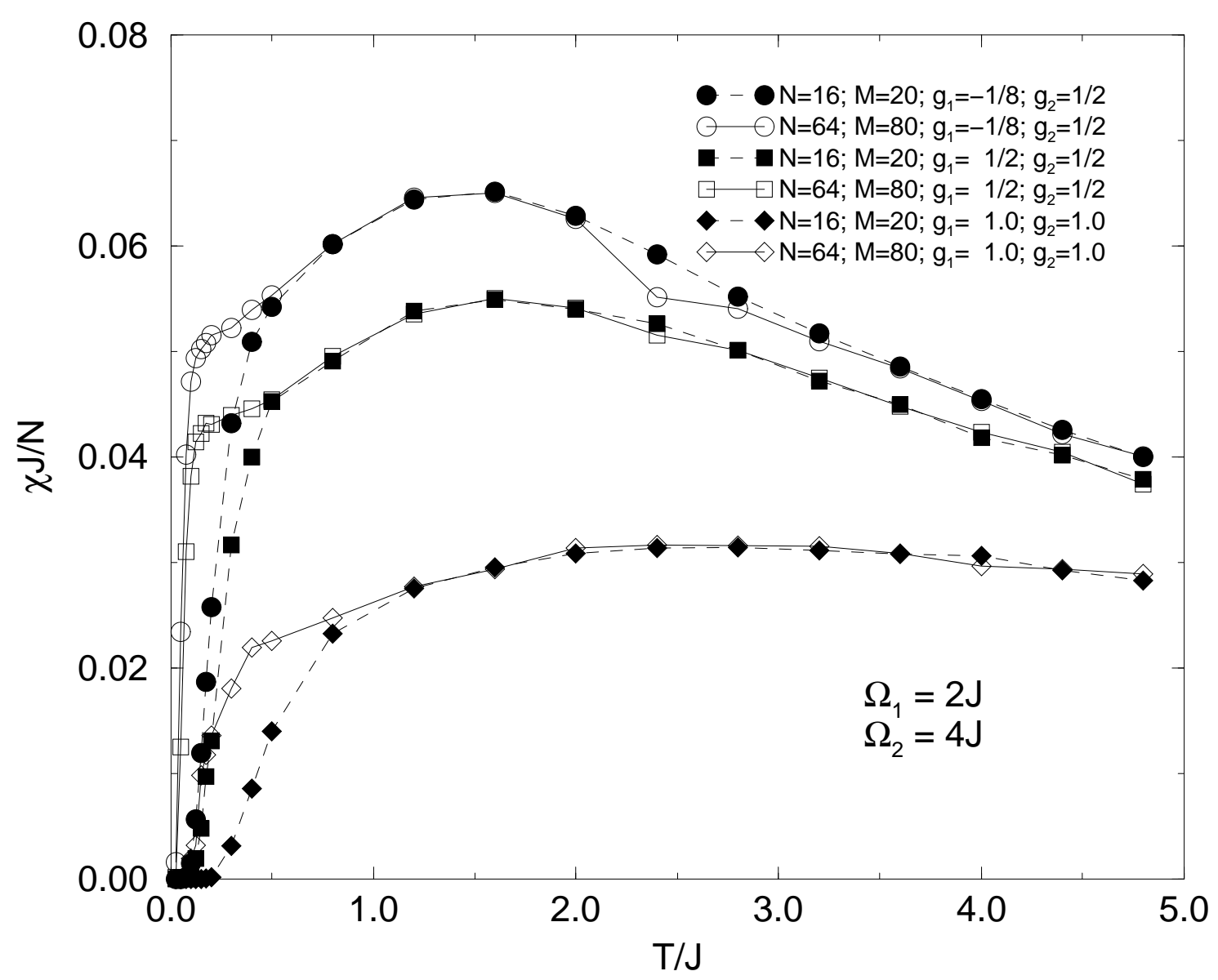

Figure 5.1: Magnetic susceptibility of three chains with the two phonon modes $\Omega_{1}=2 J$ and $\Omega_{2}=4 J$ each. The frequencies $\Omega_{1}=2 J$ and $\Omega_{2}=4 J$ and the spin-phonon coupling constants $g_{1}=-1 / 8$ and $g_{2}=1 / 2$ were chosen, because these values may resemble those of $\mathrm{CuGeO}_{3}$ (Ref. [52, 93]).

The equation for the mean phonon occupation number at finite low temperatures is,

$$
n_{i}(T \rightarrow 0)=1.0\left(\frac{g_{i} J}{\Omega_{i}}\right)^{2} \quad ; \quad i=1,2 .
$$

As we learned for the one-frequency models, the mean phonon occupation number increases with the temperature. Its behavior for temperatures $T \leq 5 J$ is shown in Figs. 5.3 and 5.4 .

From Figs. 5.1 - 5.4 we learn that the effects of the finite chain length and the finite Trotter number are roughly the same for the one-frequency and the two-frequency model. More precisely, this means that to investigate a certain quantity at a certain temperature requires least values for $N$ and $M$. These least values are about the same for the two 


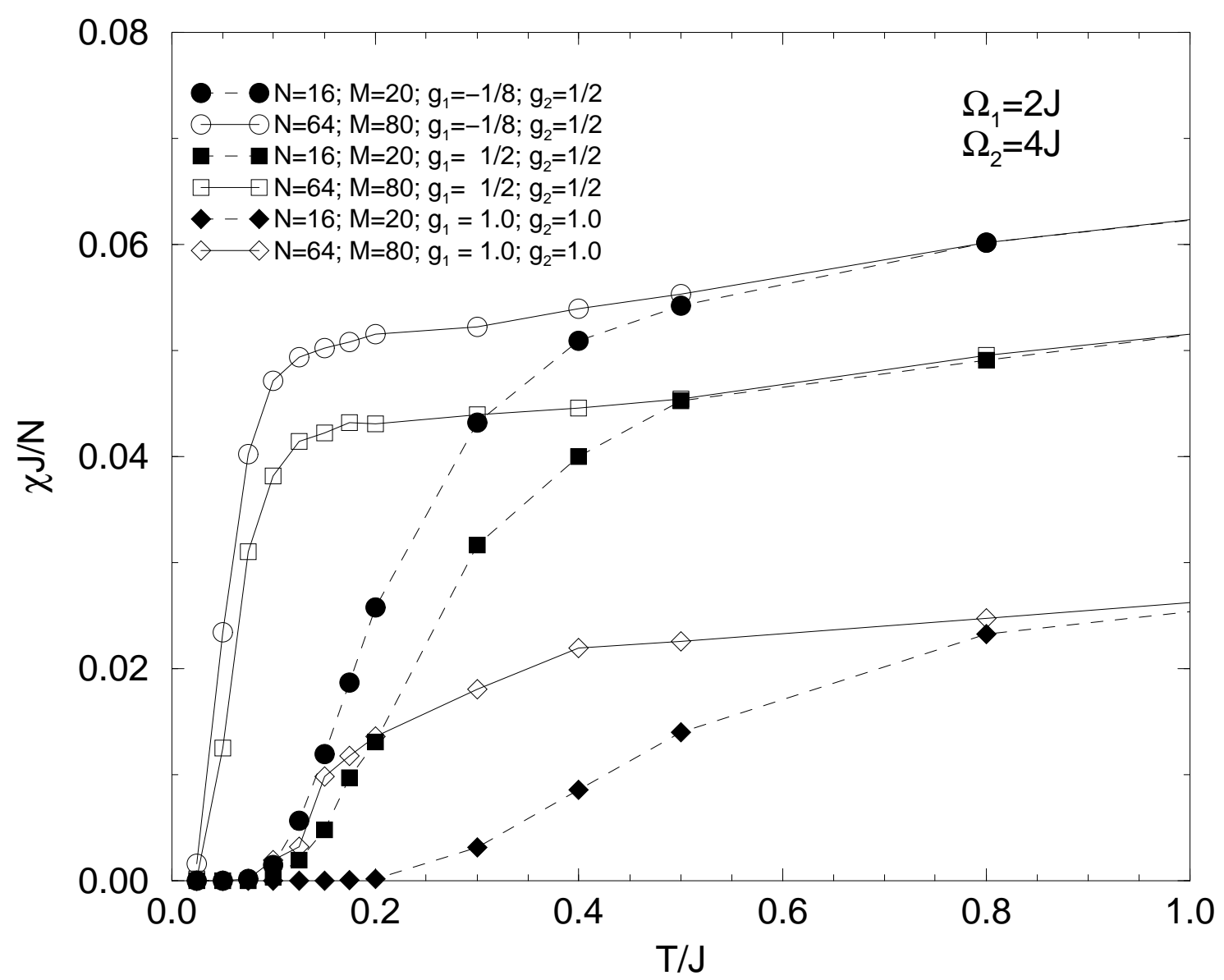

Figure 5.2: Finite-size behavior of the magnetic susceptibility of three chains with the two phonon modes $\Omega_{1}=2 J$ and $\Omega_{2}=4 J$ each.

models,

$$
N_{\text {min }} \sim M_{\text {min }} \sim 10 \beta J
$$

where $\beta$ denotes the inverse temperature.

By using the data from Figs. 4.7 and 5.5 we find $J_{\text {eff }}^{g}$ to depend on the phonon frequencies and the spin-phonon couplings according to,

$$
\frac{J_{\mathrm{eff}}^{g} / J-1}{\sum_{i=1}^{n} g_{i}^{2} J / \Omega_{i}}=1.79 \pm 0.07
$$

where $n$ is the number of phonon-frequency modes used in the model. More precisely, we confirmed this formula only for $n=1$ and $n=2$ (and special values of $g_{i}$ and $\Omega_{i}$ ), where the data of Fig. 5.5 for $n=2$ alone give the proportionality constant $1.82 \pm 0.03$. This quantitative result is a confirmation of Eq. (4.11). The generalization for arbitrary $n$ is a conjecture. 


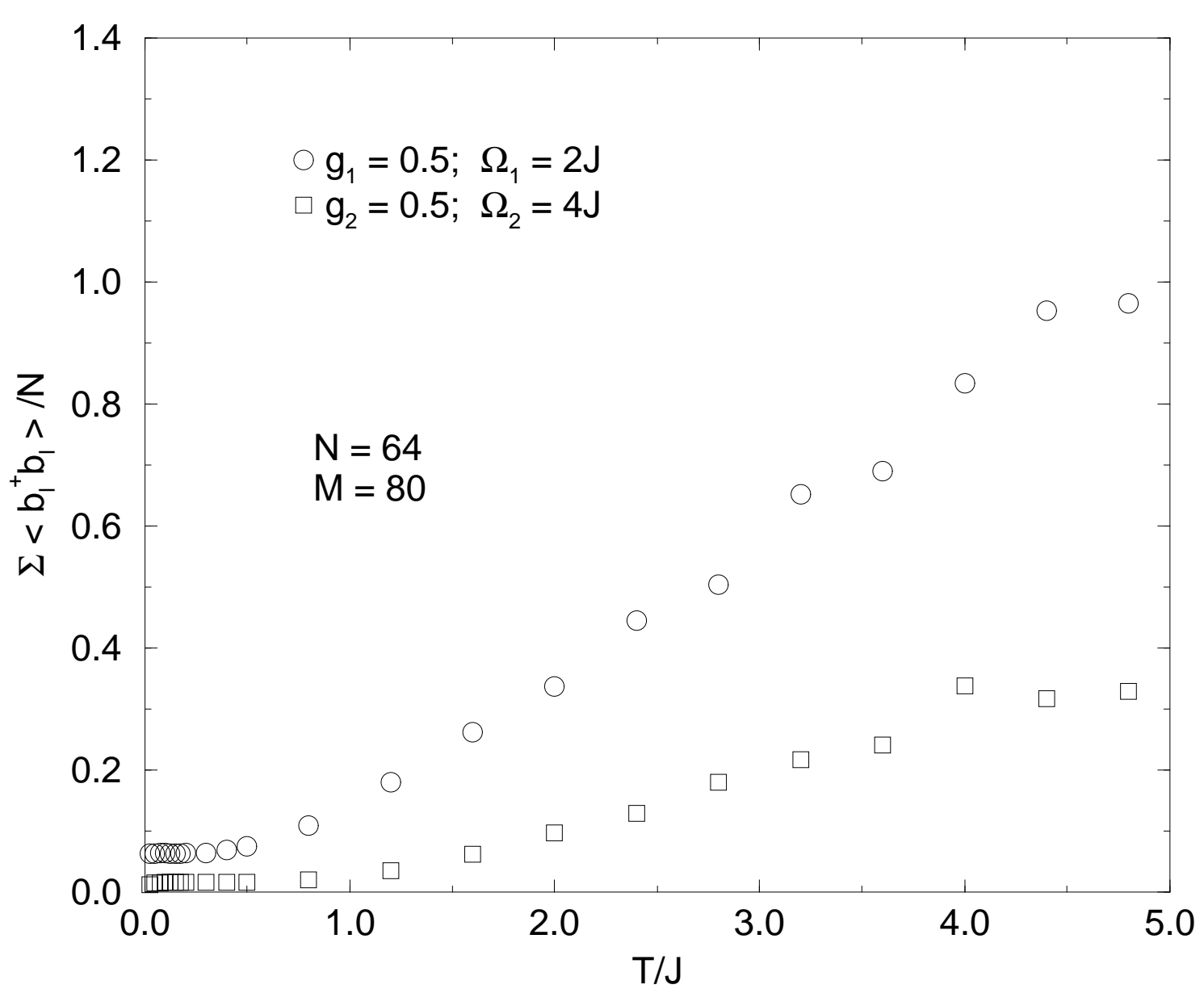

Figure 5.3: Mean phonon occupation number per site for a chain with $N=64, M=80$, $g_{1}=g_{2}=0.5, \Omega_{1}=2 J$, and $\Omega_{2}=4 J$.

Naturally, the phonons of the actual spin-Peierls materials are neither (dispersionless) Einstein phonons nor one-dimensional. Hence, only the order of magnitude of the values of $g$ and $\Omega$ of our models and those of the actual materials can be compared.

Except for the steep decline at low temperatures which is generated by the finite-size effects, the magnetic susceptibilities of the two-frequency systems we investigated can be mapped onto that of the Heisenberg chain with an effective spin-spin coupling constant. (Of course, for systems in the gapped phase one can map only the susceptibility in the high temperature region onto that of the Heisenberg chain.) By contrast, we cannot map the magnetic susceptibilities onto that of $\mathrm{CuGeO}_{3}$. The most likely explanation for this failure is that for the description of $\mathrm{CuGeO}_{3}$ frustration effects have to be considered. These effects evidently do not result from the bond phonons we examined in this study. A recent study shows that frustration effects can result from site phonons [99], as suggested by Uhrig [102]. Furthermore, it has to be shown whether these possible frustration effects 


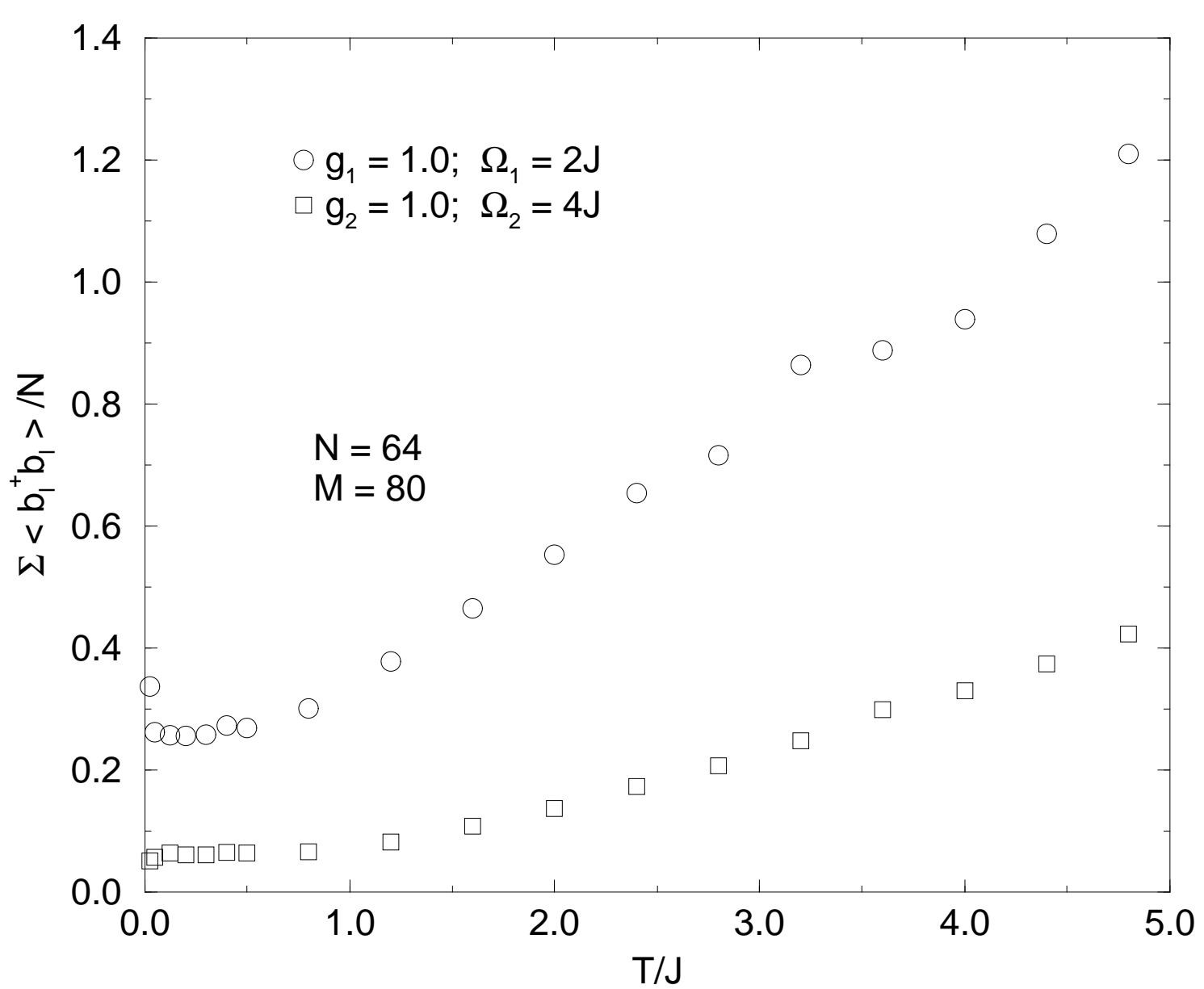

Figure 5.4: Mean phonon occupation number per site for a chain with $N=64, M=80$, $g_{1}=g_{2}=1.0, \Omega_{1}=2 J$, and $\Omega_{2}=4 J$.

can explain the thermodynamics of $\mathrm{CuGeO}_{3}$ or whether the frustration of $\mathrm{CuGeO}_{3}$ does not result from an effective spin-phonon interaction but is a manifest next-nearest neighbor spin-spin interaction. - When applying the $g-\Omega$ phase-diagram presented by Bursill et al. [8] to the one-dimensional system with site phonons on the spin-phonon parameters $g_{1}, g_{2}, \Omega_{1}, \Omega_{2}$ given in Refs. [52,93] for $\mathrm{CuGeO}_{3}$, we are attempted to conclude that the spin-phonon interaction of $\mathrm{CuGeO}_{3}$ alone is not strong enough to generate dimerization. In this preliminary approach it is assumed that one can map a two frequency mode model onto a one frequency mode model by defining $g^{2} \Omega:=g_{1}^{2} \Omega_{1}+g_{2}^{2} \Omega_{2}$. A definitive conclusion cannot be drawn until a phase-diagram for the two-frequency system has been presented and the spin-phonon coupling constants of $\mathrm{CuGeO}_{3}$ been precisely determined.

Finally, we remark that the crystal structure of the physical spin-Peierls material is rather complicated with respect to the one-dimensional Heisenberg model coupled to onedimensional Einstein phonons examined in this study. For example, for $\mathrm{CuGeO}_{3}$ the "unit 


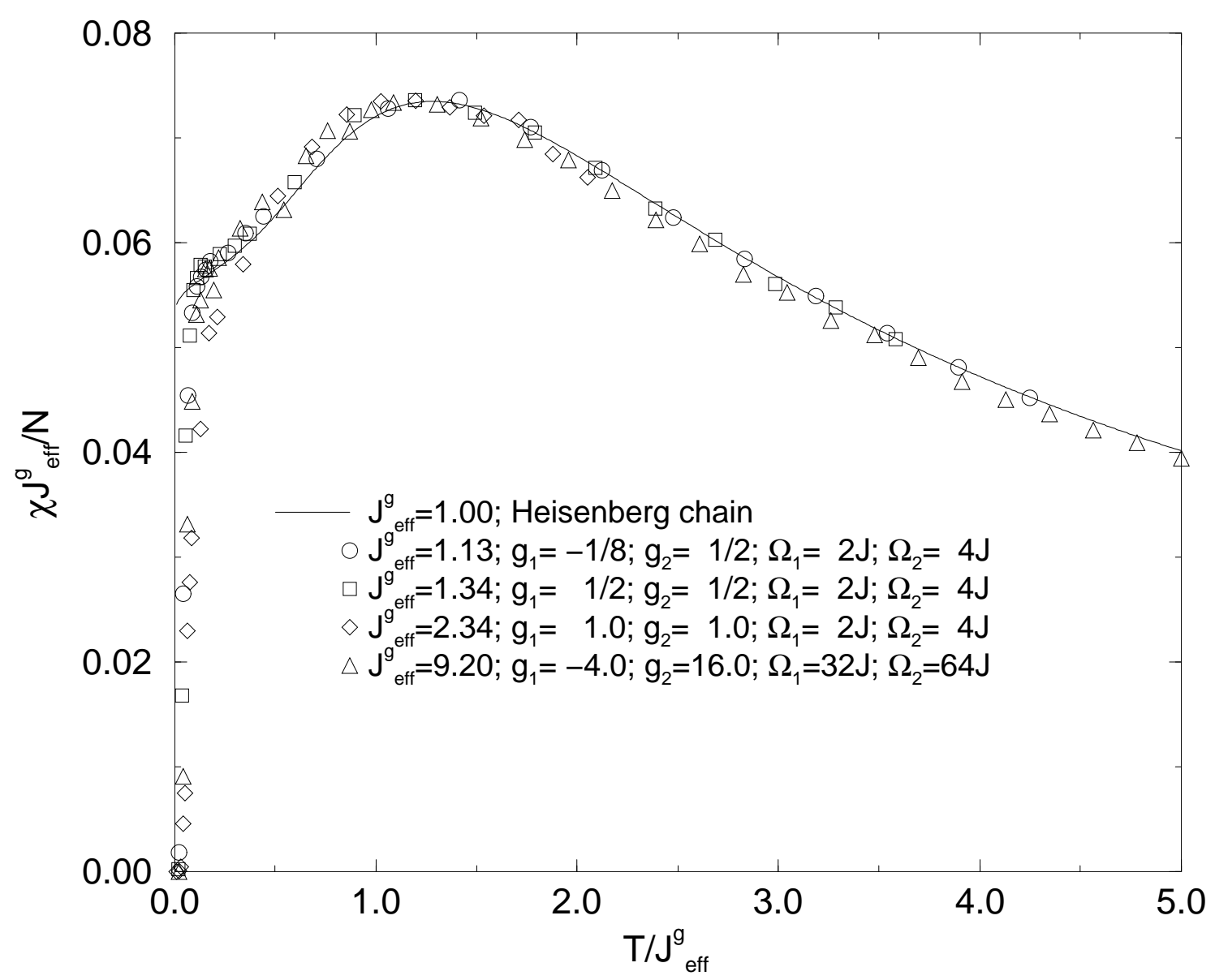

Figure 5.5: Rescaled magnetic susceptibilities $\chi\left(J, g_{1}, g_{2}, \Omega_{1}, \Omega_{2}, T\right) J_{\text {eff }}^{g} / N$ versus $T / J_{\text {eff }}^{g}$. As for the one-frequency model we can see that $J_{\text {eff }}$ is the appropriate effective spin-spin coupling to map the magnetic susceptibility of a spin-phonon chain onto a one-dimensional Heisenberg system.

cell" $a_{p} \times b_{p} \times c_{p}$ detected by Völlenkle et al. [49] turned out to be a pseudo unit cell. The actual unit cell is eight times larger, $a=2 a_{p}=9.5998 \AA, b=b_{p}=8.4665 \AA$, and $c=4 c_{p}=11.778 \AA$. This results from the behavior of the $\mathrm{GeO}_{4}$ tetraeders. A periodic change of their tilting and rotation is formed along the $c$-axis with the period of $4 c_{p}$. The directions of their rotations along the $a$-axis are in anti-phase. This results in a doubling of the pseudo unit cell along the $a$-axis [91]. Evidently, these three-dimensional phononic contributions of the lattice cells which may influence the critical temperature for the spinPeierls phase transition, cannot be described by a one-dimensional Heisenberg system which considers the monatomic lattice only. The quantum Monte Carlo investigation of $\mathrm{CuGeO}_{3}$ therefore remains a task for further investigations. The results presented in this study will hopefully be helpful for achieving this. 


\section{Chapter 6}

\section{Conclusions}

In this work we performed numerical simulations of the Heisenberg XXZ-model, the dimerized model, and the isotropic Heisenberg model coupled to Einstein phonons with either one or two frequency modes. The examined quantities included the magnetic susceptibility, the specific heat, the mean phonon occupation number, the phonon distribution, the local phonon displacement, the effective spin-spin coupling, and the spin-spin correlation.

The results obtained can be summarized as follows.

- In the continuous version of the loop algorithm the number of transition times $N_{t t}$ per site and step for the one-dimensional isotropic antiferromagnetic Heisenberg system with nearest neighbour interaction is proportional to the inverse temperature at low temperatures (see Fig. 2.12). For $T \rightarrow 0$ we find $N_{t t}=1.181,79(11) \beta J$. In the discrete case this requires the least Trotter number $M$ to be proportional to the inverse temperature. Since the spin-spin correlation length of the XXXmodel is also proportional to the inverse temperature, this means that in most cases it is reasonable to increase both the chain length $N$ and the Trotter number $M$ proportional to the inverse temperature.

- The local phonon displacement,

$$
\delta_{l}:=g\left\langle b_{l}^{\dagger}+b_{l}\right\rangle
$$

where $b_{l}^{\dagger}$ and $b_{l}$ denote the phonon creation and annihilation operators, respectively, turned out to be a useful tool for the examination of whether a system with given phonon frequency $\Omega$ and spin-phonon coupling $g$ is in the gapped or the gapless phase. By using this approach we were able to determine the phase boundary between these two phases for the XXX-chain coupled to Einstein phonons.

- The average

$$
\delta(T):=\frac{1}{N} \sum_{l=1}^{N} \delta_{l}
$$

of the local phonon displacement was found to depend on $g$ and $\Omega, \delta \propto g^{2} / \Omega$ (for fixed temperature). For temperatures $T \leq J$ the local phonon displacement is 
roughly constant (maybe slightly decreasing with growing temperature), for higher temperatures $\delta$ decreases (for fixed $g$ and $\Omega$ ).

- Usually, an effective spin-spin coupling is defined by this average phonon displacement,

$$
J_{\text {eff }}(T)=J(1+\delta) .
$$

We found that a much more useful effective spin-spin coupling $J_{\text {eff }}^{g}$ can be obtained when the magnetic susceptibility of a spin-phonon system is mapped onto the magnetic susceptibility of the pure spin system rescaled by the temperature independent $J_{\text {eff }}^{g} / J$. By examining spin-phonon systems with one phonon frequency mode we found the relation $J_{\mathrm{eff}}^{g} / J-1=c g^{2} J / \Omega$ (see Fig. 4.7). By examining spin-phonon systems with two phonon frequency modes we found the relation $J_{\text {eff }}^{g} / J-1=c\left(g_{1}^{2} J / \Omega_{1}+g_{2}^{2} J / \Omega_{2}\right)$ (see Fig. 5.5). In both cases the proprtionality constant was $c \simeq 1.8$. Evidently, the same dependence on $g^{2} / \Omega$ as for $J_{\text {eff }}(T)$ appears.

- We confirmed the appearance of the phase transition between the gapped phase and the gapless phase by using various quantities. We identified the dimerization by examining the local phonon displacement $\delta_{l}$, the spin-spin correlation $\left\langle\vec{\sigma}_{l} \cdot \vec{\sigma}_{l+1}\right\rangle$, confirmed it by the step-like behavior (in the gapped phase) of the staggered spinspin correlation $(-1)^{i-j}\left\langle S_{i}^{z} S_{j}^{z}\right\rangle$, where $|i-j|$ is the distance between two sites in units of the lattice constant $a$. Furthermore, the magnetic susceptibility showed the appearance of a spin gap at low temperatures in the gapped phase.

- The dimerization as seen in the local phonon displacement $\delta_{l}$ and the spin gap of the magnetic susceptibility $\chi$ occurred simultaneously. More precisely, gapped systems showed the periodic dimerized pattern of $\delta_{l}$ at temperatures where $\chi$ showed the spin gap. At temperatures where $\chi$ increased strongly with temperature, $\delta_{l}$ no longer showed the chain in one of the dimerized ground states, instead domain walls appeared. Above the gap temperature, $\chi$ followed the behavior of that of the Heisenberg chain with rescaled spin-spin coupling and $\delta_{l}$ did not show any dimerization.

- A remarkable transition between the low and high temperature phase occurred by examining the staggered spin-spin correlation $(-1)^{i-j}\left\langle S_{i}^{z} S_{j}^{z}\right\rangle$. In the low temperature phase (see Fig. 4.22) the correlations decrease with growing spin-phonon coupling which is analogous to the finding of the dimerized model, where the correlation is lowered with growing dimerization. The explanation is that the spin-phonon interaction perturbs the long range order which is generated by the spin-spin interaction. However, in the high temperature phase the inverse behavior occurs (see Fig. 4.23). The correlations increase with growing spin-phonon coupling. The explanation is that thermal disorder dominates while every interaction decreases the effective temperature and therefore contributes to the long range order. For $\Omega=2 J$ we found the transition temperature to be $T=0.55(5) \mathrm{J}$. 
- We found the mean phonon occupation number $n_{i}(T)$ per site to be nonzero at low temperatures for both the one-frequency and the two-frequency model. For $T \rightarrow 0$ the dependence on $g$ and $\Omega$ is: (i) $n(T)=2(g J / \Omega)^{2}$ for the one-frequency model and (ii) $n_{1}(T)=\left(g_{1} J / \Omega_{1}\right)^{2}$ and $n_{2}(T)=\left(g_{2} J / \Omega_{2}\right)^{2}$ for the two-frequency model. These equations turned out to be valid for both gapless and gapped phases. For the general case of $n_{p}$ phonon frequency modes we conjecture that,

$$
\lim _{T \rightarrow 0} n_{i}(T)=\frac{2}{n_{p}}\left(\frac{g_{i} J}{\Omega_{i}}\right)^{2} ; \quad i=1, \ldots, n_{p} .
$$

- At higher temperatures, the mean phonon number is mainly given by the formulae for free phonons shifted by the offset above. The temperature dependence of the specific heat is compatible with this finding. At high temperatures its value is given by the Dulong-Petit law and it drops to zero for $T \rightarrow 0$. However, the curve for the specific heat cannot simply be described by the sum of the contributions $c_{s}$ and $c_{p}$ of the pure spin system and the free phonon system. Especially for soft phonons the peak of $c_{s}+c_{p}$ is wiped out and shifted to higher temperatures due to the spin-phonon coupling.

- We were able to determine the correlation length $\xi$ of the correlation function $\left\langle S_{i}^{z} S_{j}^{z}\right\rangle$ of the XXX-chain coupled to phonons of one frequency mode. The examined regions of the phase diagram include (i) the gapless phase and (ii) a small region of the gapped phase near the phase boundary, where $\xi$ can be determined above the gap temperature. For both these regions we were able to fit $\xi$ in the temperature range $0.2 J \leq T \leq J$ by the formula

$$
\frac{\xi}{a}=c_{H}(T)\left(1+\frac{g^{2} J}{\Omega}\right)\left(\frac{T}{J}\right)^{-\nu},
$$

where $\nu$ turned out to depend on both $g$ and $\Omega$,

$$
\nu=1-\frac{1}{2} \frac{|g|^{3 / 2} J}{\Omega}
$$

Here $c_{H}(T)$ is the slightly temperature dependent value of the Heisenberg chain. The prefactor of $\xi$ has the structure of the effective spin-spin couplings given above and is probably explicable in these terms. The variation of $\nu$ is compatible with the fact that strong spin-phonon coupling generates dimerization and therefore decreases the correlation length at low temperatures. However, at high temperatures, $\nu$ inverses this effect. This probably explains the transition between the low and high temperature phase of the spin-spin correlation we mentioned above. We examined the formula above for one specific system in a much lower temperature region, $0.025 J \leq T \leq J$. The parameters of this system are $\Omega=2 J$ and $g=1.0$. We have shown that the formula gives a good fit for the correlation length in the entire examined temperature range. 
- The magnetic susceptibility turned out not to be a valuable quantity when trying to determine $g$ and $\Omega$ from experimental data. Furthermore, we have to note that the experimentally obtained ratio is not $\Omega / J$, but $\Omega / J_{\text {eff }}^{g}$. Hence, a phonon appearing as a hard one in units of $J_{\text {eff }}^{g}$ may actually be a soft one in units of $J$. This is probably of interest for the investigation of the spin-Peierls transition of $\mathrm{CuGeO}_{3}$. The investigation of the temperature dependence of the correlation length may turn out to be a valuable tool for determining $g$ and $\Omega$. 


\section{Bibliography}

[1] R. Calvo, M. C. G. Passeggi, M. A. Novak, O. G. Symko, S. B. Oseroff, O. R. Nascimento, and M. C. Terrile, Phys. Rev. B 43, 1074 (1991).

[2] R. E. Rapp, E. P. de Souza, H. Godfrin, and R. Calvo, J. Phys.: Condens. Matter 7, 9595 (1995).

[3] N. Motoyama, H. Eisaki, and S. Uchida, Phys. Rev. Lett. 76, 3212 (1996).

[4] M. Takigawa, N. Motoyama, H. Eisaki, and S. Uchida, Phys. Rev. Lett. 76, 4612 (1996).

[5] M. Takigawa, O. A. Starykh, A. W. Sandvik, and R. R. P. Singh, Phys. Rev. B 56, 13681 (1997).

[6] M. Hase, I. Terasaki, and K. Uchinokura, Phys. Rev. Lett. 70, 3651 (1993).

[7] A. W. Sandvik, R. R. P. Singh, and D. K. Campbell, Phys. Rev. B 56, 14510 (1997).

[8] R. J. Bursill, R. H. McKenzie, and C. J. Hamer, Phys. Rev. Lett. 83, 408 (1999).

[9] A. W. Sandvik and D. K. Campbell, Phys. Rev. Lett. 83, 195 (1999).

[10] N. D. Mermin and H. Wagner, Phys. Rev. Lett. 17, 1133 (1966).

[11] J. M. Kosterlitz and D. J. Thouless, J. Phys. C 6, 1181 (1973).

[12] W. Heisenberg, Z. Phys. 49, 619 (1928).

[13] H. A. Bethe, Z. Phys. 71, 205 (1931).

[14] L. Hulthén, Arkiv Mat. Astron. Fysik 26a, 1 (1938).

[15] R. Orbach, Phys. Rev. 112, 309 (1958).

[16] J. des Cloizeaux and J. J. Pearson, Phys. Rev. 128, 2131 (1962).

[17] J. C. Bonner and M. E. Fisher, Phys. Rev. A 135, 640 (1964).

[18] S. Eggert, I. Affleck, and M. Takahashi, Phys. Rev. Lett. 73, 332 (1994).

[19] A. Klümper, Eur. Phys. J. B 5, 677 (1998). 
[20] A. Klümper and D. C. Johnston, Phys. Rev. Lett. 84, 4701 (2000).

[21] E. Lieb, T. Schultz, and D. Mattis, Ann. Phys. 16, 407 (1961).

[22] S. Katsura, Phys. Rev. 127, 1508 (1962).

[23] C. N. Yang and C. P. Yang, Phys. Rev. 150, 321 (1966).

[24] C. N. Yang and C. P. Yang, Phys. Rev. 150, 327 (1966).

[25] C. N. Yang and C. P. Yang, Phys. Rev. 151, 258 (1966).

[26] E. H. Lieb, Phys. Rev. Lett. 18, 692 (1967).

[27] E. H. Lieb, Phys. Rev. Lett. 18, 1046 (1967).

[28] E. H. Lieb, Phys. Rev. Lett. 19, 108 (1967).

[29] F. Y. Wu, Phys. Rev. Lett. 18, 605 (1967).

[30] R. J. Baxter, Phys. Rev. Lett. 26, 832 (1971).

[31] R. J. Baxter, Phys. Rev. Lett. 26, 834 (1971).

[32] R. J. Baxter, Ann. Phys. 70, 193 (1972).

[33] R. J. Baxter, Ann. Phys. 70, 323 (1972).

[34] L. Onsager, Phys. Rev. 65, 117 (1944).

[35] C. Fan and F. Y. Wu, Phys. Rev. B 2, 723 (1970).

[36] J. D. Johnson, S. Krinsky, and B. M. McCoy, Phys. Rev. A 8, 2526 (1973).

[37] A. Klümper and J. Zittartz, Z. Phys. B 71, 495 (1988).

[38] L. A. Takhtadzhan and L. D. Fadeev, Russian Math. Surveys 34, 11 (1979).

[39] R. J. Baxter, Exactly Solved Models in Statistical Mechanics (Academic Press, London, 1982).

[40] R. E. Peierls, Quantum Theory of Solids (Oxford Univ. Press, Oxford, 1955), pp. $108-112$.

[41] H. M. McConnell und R. Lynden-Bell, J. Chem. Phys. 36, 2393 (1962).

[42] E. Pytte, Phys. Rev. B 10, 4637 (1974).

[43] M. C. Cross und D. S. Fisher, Phys. Rev. B 19, 402 (1979).

[44] J. W. Bray, H. R. Hart, L. V. Interrante, I. S. Jacobs, J. S. Kasper, G. D. Watkins, S. H. Wee, and J. C. Bonner, Phys. Rev. Lett. 35, 744 (1975). 
[45] I. S. Jacobs, J. W. Bray, H. R. Hart, L. V. Interrante, J. S. Kasper, G. D. Watkins, D. E. Prober, and J. C. Bonner, Phys. Rev. B 14, 3036 (1976).

[46] G. Castilla, S. Chakravarty, and V. J. Emery, Phys. Rev. Lett. 75, 1823 (1995).

[47] J. Riera and A. Dobry, Phys. Rev. B 51, 16098 (1995).

[48] K. Fabricius, A. Klümper, U. Löw, B. Büchner, T. Lorenz, G. Dhalenne, and A. Revcolevschi, Phys. Rev. B 57, 1102 (1998).

[49] H. Völlenkle, A. Wittmann, and H. Nowotny, Monatsh. Chem. 98, 1352 (1967).

[50] M. Braden, G. Wilkendorf, J. Lorenzana, M. Ain, G. J. McIntyre, M. Behruzi, G. Heger, G. Dhalenne, and A. Revcolevschi, Phys. Rev. B 54, 1105 (1996).

[51] M. Braden, B. Hennion, W. Reichardt, G. Dhalenne, and A. Revcolevschi, Phys. Rev. Lett. 80, 3634 (1998).

[52] R. Werner, C. Gros, and M. Braden, Phys. Rev. B 59, 14356 (1999).

[53] M. Isobe and Y. Ueda, J. Phys. Soc. Japan 65, 1178 (1996).

[54] Y. Fujii, H. Nakao, T. Yosihama, M. Nishi, K. Nakajima, K. Kakurai, M. Isobe, Y. Ueda, and H. Sawa, J. Phys. Soc. Japan 66, 326 (1997).

[55] T. Ohama, M. Isobe, H. Yasuoka, and Y. Ueda, J. Phys. Soc. Japan 66, 545 (1997).

[56] H. Smolinski, C. Gros, W. Weber, U. Peuchert, G. Roth, M. Weiden, and C. Geibel, Phys. Rev. Lett. 80, 5164 (1998).

[57] H. Nakao, K. Ohwada, N. Takesue, Y. Fujii, M. Isobe, Y. Ueda, M. v. Zimmermann, J. P. Hill, D. Gibbs, J. C. Woicik, I. Koyama, and Y. Murakami, Phys. Rev. Lett. 85, 4349 (2000).

[58] M. Fischer, P. Lemmens, G. Els, G. Güntherodt, E. Ya. Sherman, E. Morre, C. Geibel, and F. Steglich, Phys. Rev. B 60, 7284 (1999).

[59] Y. Fagot-Revurat, M. Mehring, and R. K. Kremer, Phys. Rev. Lett. 84, 4176 (2000).

[60] M. Suzuki, Comm. Math. Phys. 51, 183 (1976).

[61] M. Suzuki, S. Miyashita, and A. Kuroda, Prog. Theor. Phys. 58, 1377 (1977).

[62] J. E. Hirsch, D. J. Scalapino, R. L. Sugar, and R. Blancenbecler, Phys. Rev. Lett. 47, 1628 (1981).

[63] J. E. Hirsch and E. Fradkin, Phys. Rev. Lett. 49, 402 (1982).

[64] J. E. Hirsch, R. L. Sugar, D. J. Scalapino, and R. Blancenbecler, Phys. Rev. B 26, 5033 (1982). 
[65] E. Fradkin and J. E. Hirsch, Phys. Rev. B 27, 1680 (1983).

[66] J. E. Hirsch and E. Fradkin, Phys. Rev. B 27, 4302 (1983).

[67] J. E. Hirsch, Phys. Rev. Lett. 51, 296 (1983).

[68] M. Marcu and A. Wiesler, J. Phys. A 18, 2479 (1985).

[69] R. H. Swendsen and J.-S. Wang, Phys. Rev. Lett. 58, 86 (1987).

[70] U. Wolff, Nucl. Phys. B 322, 759 (1989).

[71] H.-Q. Ding and M. S. Makivić, Phys. Rev. Lett. 64, 1449 (1990).

[72] M. S. Makivić and H.-Q. Ding, Phys. Rev. B 43, 3562 (1991).

[73] D. Kandel and E. Domany, Phys. Rev. B 43, 8539 (1991).

[74] U.-J. Wiese and H.-P. Ying, Phys. Lett. A 168, 143 (1992).

[75] U.-J. Wiese and H.-P. Ying, Nucl. Phys. B (Proc. Suppl.) 30, 281 (1993).

[76] H.-P. Ying, U.-J. Wiese, and D.-R. Ji, Phys. Lett. A 183, 441 (1993).

[77] U.-J. Wiese and H.-P. Ying, Z. Phys. B 93, 147 (1994).

[78] H. G. Evertz, G. Lana, and M. Marcu, Phys. Rev. Lett. 70, 875 (1993).

[79] H. G. Evertz and M. Marcu, Nucl. Phys. B (Proc. Suppl.) 30, 277 (1993).

[80] H. G. Evertz, in Numerical Methods for Lattice Quantum Many-Body Problems, edited by D. J. Scalapino (Addison Wesley, Reading), to be published (= condmat/9707221).

[81] B. B. Beard and U. J. Wiese, Phys. Rev. Lett. 77, 5130 (1996).

[82] F. Matsubara, S. Inawashiro, and H. Ohhara, J. Phys.: Condens. Matter 3, 1815 (1991).

[83] P. M. Duxbury, J. Oitmaa, M. N. Barber, A. van der Bilt, K. O. Joung, and R. L. Carlin, Phys. Rev. B 24, 5149 (1981).

[84] H. Yoshizawa, G. Shirane, H. Shiba, and K. Hirakawa, Phys. Rev. B 28, 3904 (1983).

[85] D. Augier, D. Poilblanc, S. Haas, A. Delia, and E. Dagotto, Phys. Rev. B 56, 5732 (1997).

[86] D. Augier and D. Poilblanc, Eur. Phys. J. B 1, 19 (1998).

[87] E. Sorensen, I. Affleck, D. Augier, and D. Poilblanc, Phys. Rev. B 58, 14701 (1998).

[88] P. Hansen, D. Augier, J. Riera, and D. Poilblanc, Phys. Rev. B 59, 13557 (1999). 
[89] D. Augier, J. Riera, and D. Poilblanc, Phys. Rev. B 61, 6741 (2000).

[90] R. Chitra, S. Pati, H. R. Krishnamurthy, D. Sen, and S. Ramasesha, Phys. Rev. B 52, 6581 (1995).

[91] M. Hidaka, M. Hatae, I. Yamada, M. Nishi, and J. Akimitsu, J. Phys.: Condens. Matter 9, 809 (1997).

[92] M. Braden, B. Hennion,P. Pfeuty, G. Dhalenne, and A. Revcolevschi, Phys. Rev. Lett. 83, 1858 (1999).

[93] C. Gros and R. Werner, Phys. Rev. B 58, 14677 (1998).

[94] J. P. Pouget, L. P. Regnault, M. Ain, B. Hennion, J. P. Renard, P. Veillet, G. Dhalenne, and A. Revcolevschi, Phys. Rev. Lett. 72, 4037 (1994).

[95] D. Augier, P. Hansen, D. Poilblanc, J. Riera, and E. Sorensen, cond-mat/9807265.

[96] D. Augier, D. Poilblanc, E. Sorensen, and I. Affleck, Phys. Rev. B 58, 9110 (1998).

[97] A. Weiße, G. Wellein, and H. Fehske, Phys. Rev. B 60, 6566 (1999).

[98] R. W. Kühne and U. Löw, Phys. Rev. B 60, 12125 (1999).

[99] C. Raas, Effektive Spinmodelle für Spin-Phonon-Ketten mittels Flußgleichungen, Diploma thesis (Universität zu Köln, 2000).

[100] R. Fehrenbacher, Phys. Rev. B 49, 12230 (1994).

[101] R. Fehrenbacher, Phys. Rev. Lett. 77, 2288 (1996).

[102] G. S. Uhrig, Phys. Rev. B. 57, R14004 (1998).

[103] M. Suzuki, Phys. Rev. B 31, 2957 (1985).

[104] M. Marcu, J. Müller, and F. K. Schmatzer, Phys. Lett. A 116, 447 (1986).

[105] M. Marcu, J. Müller, and F. K. Schmatzer, J. Phys. A 18, 3189 (1985).

[106] K. Fabricius and U. Löw, Phys. Rev. B 57, 13371 (1998).

[107] G. S. Uhrig, Phys. Rev. Lett. 79, 163 (1997).

[108] G. Wellein, H. Fehske, and A. P. Kampf, Phys. Rev. Lett. 81, 3956 (1998).

[109] M. Weiden, J. Köhler, G. Sparn, M. Köppen, M. Lang, C. Geibel, and F. Steglich, Z. Phys. B 98, 167 (1995).

[110] M. Greven, R. J. Birgeneau, Y. Endoh, M. A. Kastner, B. Keimer, M. Matsuda, G. Shirane, and T. R. Thurston, Phys. Rev. Lett. 72, 1096 (1994). 
[111] M. Greven, R. J. Birgeneau, Y. Endoh, M. A. Kastner, M. Matsuda, and G. Shirane, Z. Phys. B 96, 465 (1995).

[112] K. Fabricius, U. Löw, and K.-H. Mütter, Phys. Rev. B. 51, 8270 (1995).

[113] J. L. Cardy, Scaling and Renormalization in Statistical Physics (Cambridge Univ. Press, Cambridge, 1996), p. 7.

[114] A. Klümper, Z. Phys. B 91, 507 (1993). 


\section{Acknowledgements}

This work in its present form was made possible by the support I enjoyed in many ways. I thank especially Ute Löw and Andreas Klümper for the intensive supervision including countless fruitful discussions, help, and encouragement. Furthermore, I thank Christiane Hochkeppel, Frank Schlesener, and Andreas Fledderjohann for valuable discussions. Andreas Klümper provided me with the exact data of the magnetic susceptibility, specific heat, internal energy, and correlation length of the isotropic Heisenberg model.

The financial support of the Deutsche Forschungsgemeinschaft, the Graduiertenkolleg Wuppertal, and the Graduiertenkolleg Dortmund is acknowledged. 